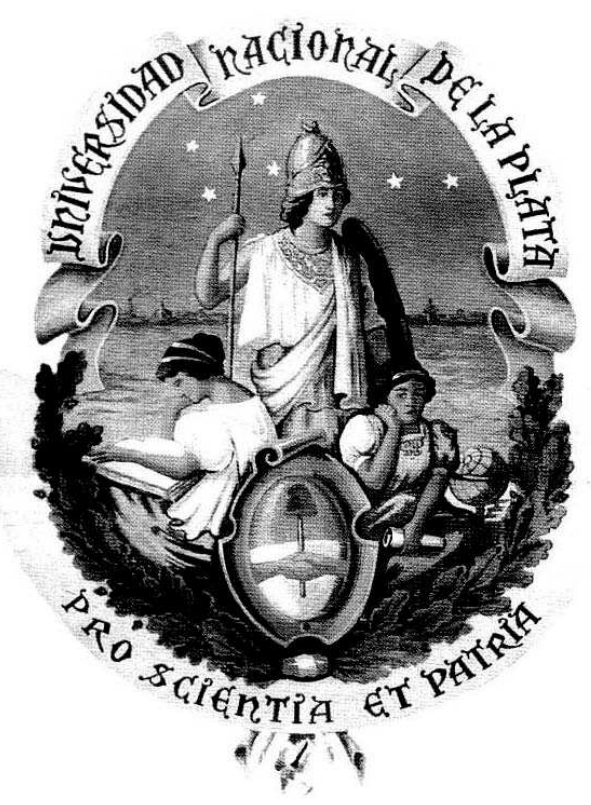

Universidad Nacional de La Plata

Facultad de Ciencias Astronómicas y Geofísicas

Tesis para obtener el grado académico de

Doctor en Astronomía:

\title{
EvoluCión COLISIONAL Y DINÁMICA DE PEQUEÑOS PLANETAS
}

Lic. Gonzalo C. de Elía

Director: Dr. Adrián Brunini 

Dedicada a mi mamá Alicia, mi papá Carlos, mi hermano Sebastián, y mi novia Macarena, por ayudarme cada día a ser una mejor persona. 



\section{Prefacio}

Esta Tesis es presentada como parte de los requisitos para obtener el grado académico de Doctor en Astronomía de la Universidad Nacional de La Plata. La misma contiene los estudios desarrollados bajo la dirección del Dr. Adrián Brunini y en colaboración con investigadores del Grupo de Ciencias Planetarias de la Facultad de Ciencias Astronómicas y Geofísicas de La Plata, entre los años 2004 y 2008.

Gonzalo Carlos de Elía gdeelia@fcaglp.unlp.edu.ar La Plata, Febrero de 2009 



\section{Originalidad}

Las ideas, desarrollos, programas de computación y resultados obtenidos son exclusivos del autor y del director de la Tesis, salvo que se haga expresa mención al respecto. La realización de este trabajo nos ha permitido llevar a cabo tres publicaciones en la revista Astronomy \& Astrophysics, las cuales se detallan a continuación:

- Collisional and dynamical evolution of the Main Belt and NEA population, de Elía, G. C., \& Brunini, A. 2007, A\&A, 466, 1159-1177.

- Collisional and dynamical evolution of the $L_{4}$ Trojan asteroids, de Elía, G. C., \& Brunini, A. 2007, A\&A, 475, 375-389.

- Collisional and dynamical evolution of Plutinos, de Elía, G. C., Brunini, A., \& Di Sisto, R. P. 2008, A\&A, 490, 835-842. 



\section{Agradecimientos}

Una vez escuché en una película que alguien decía "Las personas no somos islas ...". Definitivamente, esta es una frase que se ajusta perfectamente a este momento. Si hay algo de lo que estoy convencido es que ningún logro es personal. Siempre hay personas que hacen que nuestra vida sea más placentera, que nos brindan su ayuda sin esperar nada a cambio, que nos hacen superar nuestras dificultades más fácilmente, que disfrutan de nuestras alegrías, que nos transmiten paz. Gracias a Dios tengo mucha de esta clase de gente en mi vida y no quiero olvidarme de ellos en este momento tan especial.

En primer lugar, quiero darle gracias a Adrián Brunini. Él ha sido el director de mi tesis y me ha transmitido muchas cosas importantes. He valorado mucho sus consejos a lo largo de todo este tiempo y nunca me voy a olvidar que si hoy en día estoy trabajando en esa oficina maravillosa es gracias a Él. Gracias por tu apoyo y tu ayuda, Adrián. He aprendido mucho. Todos estos años han representado una gran experiencia para mi. Gracias por todo.

También quiero agradecerle a Héctor Viturro. Gracias por la administración y mantenimiento de las computadoras con las cuales trabajamos y desarrollamos todas las simulaciones numéricas analizadas en esta tesis.

Quiero darle las gracias también a Romina Di Sisto. Ella es una excelente profesional pero por sobre todas las cosas es una gran persona. Gracias por tu amistad, por tu generosidad, $\mathrm{y}$ por tu paciencia. Y fundamentalmente gracias por ser tan amable y mostrar una gran predisposición en cada cosa que te pido. Me has ayudado muchísimo, Romina Paula. Me da mucha satisfacción poder trabajar con vos y espero poder seguir haciéndolo por mucho tiempo más. Una y mil veces, Gracias !!! ...

También quiero agradecerle a Pablo Santamaría. Desde el año 2004 creo haberle hecho todas las preguntas posibles sobre cuestiones numéricas y de computadoras ... y sinceramente no recuerdo ninguna situación en la cual Pablo me haya dicho "... después te digo ...". Gracias por tu amistad y por esa gran predisposición que tenés para transmitir lo que sabés. Una vez te lo dije. A veces es difícil encontrar personas que sepan mucho de algo y que a la vez sean bien predispuestas. Vos, Pablo, sos una de esas personas. Gracias otra vez.

Quiero darle las gracias también a Lorena Dirani. Gracias por tu amistad, Lore. Por esas tardes de mate y largas conversaciones, por tus buenos consejos y por la buena onda. Nunca me voy a olvidar de la ayuda que me dieron junto con Fede (Bareilles) cuando mi compu no andaba. Yo no me olvido. Gracias sinceras por todo el apoyo.

Quiero agradecerle también a Jorge Panei. Un fenómeno, JAP !!!. Aun cuando había dejado de ser Secretario Académico de la Facultad, me seguía contestando preguntas sobre infinidad de temas. Gracias por tu amistad, Jorge !!!. Sos una gran persona, un gran amigo. Siempre con buena onda y mirando el lado positivo. Y además, Pincha fanático como yo !!! .... Grande Jorge !!! ...

También quiero agradecerle enormemente a mi gran amiga Andrea Fortier. Gracias por 
todo, Júpiter !!!!!. Sos una gran persona, una gran amiga. Hemos recorrido un gran camino juntos y a lo largo de todos estos años hemos construido una amistad que durará por siempre. Gracias por todos los momentos compartidos, por todo el apoyo, y por hacerme sentir que, estés donde estés, siempre podré contar con vos. Muchas Gracias por todo, amiga !!! ...

Quiero agradecerle también a mi gran amigo Marcelo Miller Bertolami. Marcelino, sos un fenómeno !!! ... Un profesional super responsable pero por sobre todas las cosas una gran persona. Siempre me has ayudado ... siempre ... no recuerdo una oportunidad en la que me hayas dicho "... ahora no puedo ...". Si hay una persona inteligente, responsable y siempre bien predispuesta para ayudar al otro, ese es Marcelo. Gracias por el apoyo y la amistad incondicional de todos estos años. Muchas Gracias por todo, pibe !!! ...

Quiero darle las gracias también a Mariana Orellana, Anahí Granada, Cecilia Fariña, Claudia Scóccola e Ileana Andruchow. Gracias por su amistad y por el apoyo que me brindaron a lo largo de todos estos años.

En el año 2005 tuve la oportunidad de ir a la Escuela del Vaticano y vivir una experiencia inolvidable con mucha gente especial. Quiero darle las gracias por todo lo que me brindaron a George Coyne, Chris Corbally, José Funes, Celeste Parisi y Dany Stasyszyn, Klara Hlouchova y David Hlouch, Katarina Miljkovic, Ella Carlson, Marcela Ewert, Brenda Miranda, Eder Martioli, Bernardo Borges, Juan José Downes, y Nikolai Nikolov. Somos parte de algo especial. Gracias por su amistad y los grandes momentos compartidos.

Para finalizar, quiero darle las gracias a mi familia. Esto es, a mi mamá Alicia, mi papá Carlos y mi hermano Sebastián. Gracias por mostrarme a través de su ejemplo como es ser una persona de bien. Gracias por educarme sanamente, por inculcarme buenos valores, por enseñarme que las cosas se hacen con responsabilidad, esfuerzo y sacrificio, y que la palabra vale más que cualquier documento. Gracias por darme la oportunidad de estudiar, por bancarme durante toda la carrera, por llegar a casa y recibirme festejando mis alegrías y amortiguando mis dificultades. Tengo la mejor familia del mundo. Y también sé muy bien que mi abuela Navía y el Baba están juntos disfrutando desde allí arriba. No me va a alcanzar la vida para agradecerles por todo lo que me han dado. Y estén seguros de una cosa ... este título no es mío ... es nuestro.

Finalmente, quiero darle las gracias a Macarena Zanardi. Gracias por todo, linda. Por darme tu amor incondicional y hacerme una mejor persona. Gracias por escucharme, por sonreír cada día con dulzura, y por hacer que todo sea de colores. Gracias por soñar una vida juntos y ser tan especial. Te quiero con el alma, mi ángel. Eternamente Gracias ...

Termino con una frase de Vince Lombardi que dice "... Creo firmemente que el mejor instante del hombre, la mayor satisfacción de todo lo que ha querido es cuando ha entregado su corazón a una buena causa y yace exhausto en el campo de batalla, victorioso ...". Algo de verdad debe tener. Después de mucho tiempo y sacrificio, hemos cruzado una meta ... y realmente me siento muy feliz.

Gonzalo Carlos de Elía 


\section{Resumen}

En esta Tesis se presentan resultados concernientes a la evolución colisional y dinámica de las principales poblaciones de pequeños planetas del Sistema Solar. Nuestro análisis incluye a los asteroides del Cinturón Principal, los asteroides cercanos a la Tierra (NEAs), los Troyanos de Júpiter asociados al punto de equilibrio $L_{4}$, y los Plutinos, residentes en la resonancia de movimientos medios 3:2 con Neptuno.

Para realizar esta tarea, desarrollamos un código numérico estadístico de evolución colisional, capaz de incluir las principales características dinámicas de la población de pequeños cuerpos bajo estudio. Dicho algoritmo puede ser utilizado para analizar la evolución de cualquier población de pequeños planetas, independientemente de su posición en el Sistema Solar.

Inicialmente, estudiamos la evolución colisional y dinámica de los asteroides del Cinturón Principal y NEAs de manera simultánea. Si bien los procesos colisionales resultan ser relevantes, el modelo dinámico juega un rol clave ya que es el responsable de conectar el Cinturón Principal de asteroides con la población de NEAs. Nuestro algoritmo incluye los efectos dinámicos generados por resonancias poderosas, resonancias difusivas y el efecto Yarkovsky, siendo este último el resultado de un mecanismo de radiación que actúa sobre los cuerpos rotantes del Sistema Solar. Nuestro estudio ha logrado obtener resultados consistentes con diversos observables tales como las distribuciones de tamaños asociadas a las poblaciones del Cinturón Principal y NEAs, sus masas actuales, el número de grandes familias, la historia colisional del asteroide (4) Vesta y el tiempo de exposición a los rayos cósmicos de los meteoritos. Por otra parte, hemos analizado la mezcla taxonómica de asteroides en el Cinturón Principal. Nuestras simulaciones sugieren que dicha mezcla de material no puede ser explicada a partir del intercambio colisional de masa, debiendo ser un rasgo primordial de esta estructura. Además, nuestro trabajo nos ha permitido realizar determinaciones cuantitativas concernientes al origen de los NEAs. En efecto, nuestros estudios indican que el $\sim 94 \%$ de los NEAs provienen de la región del Cinturón Principal interior a las $2.8 \mathrm{UA}$, mientras que sólo el $6 \%$ restante proviene del Cinturón Exterior.

Posteriormente, analizamos la evolución colisional y dinámica de los Troyanos jovianos del punto de equilibrio $L_{4}$, teniendo en cuenta las regiones de estabilidad e inestabilidad de la resonancia 1:1 con Júpiter. Nuestro estudio nos ha permitido derivar estimaciones sobre la distribución de tamaños de estos objetos, sus tiempos de vida colisionales, el número de grandes familias de Troyanos, la emisión del polvo y las tasas de eyección de fragmentos colisionales. Los resultados concernientes a la distribución de tamaños y el número de familias son consistentes con los datos observacionales. Por otra parte, encontramos que los Troyanos de mayor tamaño resultan ser objetos primordiales de esta población. Además, nuestros análisis indican que la luminosidad del polvo en el enjambre joviano $L_{4}$ resulta ser comparable a la luminosidad del polvo en el Sistema Solar interior y, al menos, un orden de magnitud más baja que la luminosidad del polvo asociado al Sistema Solar exterior. Luego, analizando las 
tasas de eyección de fragmentos colisionales, inferimos que la contribución de los Troyanos sobre las poblaciones actuales de Centauros y Cometas de la Familia de Júpiter es despreciable, comparada con aquella del Disco Dispersado de la Región Transneptuniana, considerado como fuente primaria.

Finalmente, estudiamos la evolución colisional y dinámica de los Plutinos, teniendo en cuenta las regiones de estabilidad e inestabilidad dinámica de la resonancia de movimientos medios 3:2 con Neptuno. En este trabajo analizamos la distribución de tamaños de estos objetos, infiriendo que la existencia de un quiebre a un diámetro de 40-80 km debería ser una característica primordial de esta población. Por otra parte, nuestras simulaciones nos permiten obtener estimaciones sobre los tiempos de vida colisionales de los Plutinos, el número de grandes familias y las tasas de eyección de fragmentos. Nuestros resultados indican que es necesario especificar el número de objetos del tamaño de Plutón presentes en esta población, con el fin de computar el número de familias y determinar si esta región resonante puede ser considerada una fuente importante de Cometas Eclípticos, comparable al Disco Dispersado de la Región Transneptuniana. 


\section{Índice general}

Prefacio $\quad$ V

\begin{tabular}{ll|l|l} 
Originalidad & VII
\end{tabular}

$\begin{array}{lll}\text { Agradecimientos } & \text { IX }\end{array}$

$\begin{array}{lll}\text { Resumen } & \text { XI }\end{array}$

$\begin{array}{ll}\text { 1. Introducción } & 3\end{array}$

2. Perspectiva Histórica $\quad 9$

2.1. El Descubrimiento de Ceres y el Origen de los Asteroides . . . . . . . . . . . . 9

2.2. Los Troyanos en el Sistema Solar . . . . . . . . . . . . . . . . . . . 12

2.3. La Región Transneptuniana . . . . . . . . . . . . . . . . . . . . . . . 14

2.3.1. El Descubrimiento de Plutón . . . . . . . . . . . . . . . . 14

2.3.2. Contribuciones de Edgeworth y Kuiper . . . . . . . . . . . . . 16

2.3.3. Contribuciones Modernas . . . . . . . . . . . . . . . . 18

2.4. El Descubrimiento de Eris (2003 UB313) y la Nueva Definición de Planeta . . 19

3. Generalidades 23

3.1. Estructura Dinámica y Distribución Orbital . . . . . . . . . . . . . 23

3.1.1. Resonancias en el Sistema Solar . . . . . . . . . . . . . . . . . 23

3.1.2. Conexión Dinámica entre los Asteroides del Cinturón Principal y los NEOs .............................. 27

3.1.3. Troyanos Jovianos: Propiedades Orbitales y Características Dinámicas 33

3.1.4. Estructura Dinámica de la Región Transneptuniana . . . . . . . . . . 34

3.1.5. Cometas . . . . . . . . . . . . . . . . 47

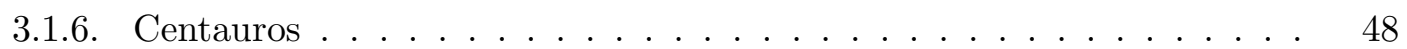

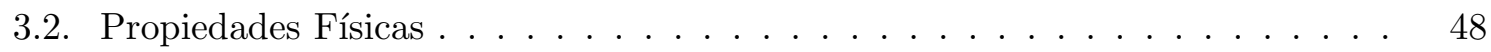

3.2.1. Asteroides del Cinturón Principal y NEOs . . . . . . . . . . . . . . . 49

3.2.2. Troyanos Jovianos . . . . . . . . . . . . . . . . 53

3.2.3. Centauros y Objetos de la Región Transneptuniana . . . . . . . . . . . 56

3.3. Colisiones entre Asteroides - Leyes de Impacto . . . . . . . . . . . . . . 61

4. Modelo Colisional $\quad 65$

4.1. Definiciones . . . . . . . . . . . . . . . . . . . . . . . . . . . . . . 65

4.2. Impactos Catastróficos . . . . . . . . . . . . . . . . . 66 
4.3. Impactos Craterizantes . . . . . . . . . . . . . . . . . . . 67

4.4. Escape y Reacumulación de Fragmentos . . . . . . . . . . . . . . . . . 68

$\begin{array}{ll}\text { 5. Asteroides del Cinturón Principal y NEAs } & \mathbf{7 3}\end{array}$

5.1. Introducción . . . . . . . . . . . . . . . . . . . 73

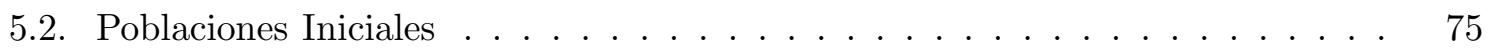

5.3. Límites de las Resonancias $\nu_{6}, 3: 1$ y $5: 2 \ldots \ldots \ldots \ldots$

5.4. El Rol del Efecto Yarkovsky . . . . . . . . . . . . . . . . . 83

5.4.1. Consideraciones Generales . . . . . . . . . . . . . . . . . . 83

5.4.2. Modelo Matemático . . . . . . . . . . . . . . . . 86

5.5. Parámetros Colisionales . . . . . . . . . . . . . . . . . . 92

5.5.1. Probabilidad Intrínseca de Colisión y Velocidad de Impacto . . . . . . 92

5.5.2. Leyes de Impacto . . . . . . . . . . . . . . . . . . . . . . . 94

5.6. Modelo Numérico de Evolución Colisional y Dinámica . . . . . . . . . . . . 96

5.7. Resultados . . . . . . . . . . . . . . . . . . . . 100

5.7.1. Distribuciones de Tamaños del Cinturón Principal de Asteroides y NEAs100

5.7.2. Remoción de Asteroides desde el Cinturón Principal . . . . . . . . . . 103

5.7.3. Tiempo de Exposición a los Rayos Cósmicos de los Meteoritos . . . . 105

5.7.4. Historia Colisional del Asteroide (4) Vesta . . . . . . . . . . . . . . . . . 105

5.7.5. Familias de Asteroides . . . . . . . . . . . . . . . . . . . . 109

5.7.6. Mezcla de Clases Taxonómicas - Discusión . . . . . . . . . . . . . . . . 111

5.7.7. Origen de los NEAs . . . . . . . . . . . . . . 112

6. Troyanos de Júpiter 115

6.1. Introducción . . . . . . . . . . . . . . . . . . . . 115

6.2. Población Inicial . . . . . . . . . . . . . . . . . . . . . 118

6.3. Tratamiento Dinámico . . . . . . . . . . . . . . . . . . . 121

6.4. Parámetros Colisionales y Relación con Otras Poblaciones . . . . . . . . . . . 123

6.4.1. Probabilidad Intrínseca de Colisión y Velocidad de Impacto . . . . . . 123

6.4.2. Relaciones con Hildas y Cometas de la Familia de Júpiter . . . . . . . 124

6.4.3. Leyes de Impacto . . . . . . . . . . . . . . . . . . . . . . . . . 124

6.5. Modelo Numérico de Evolución Colisional y Dinámica . . . . . . . . . . . . . 126

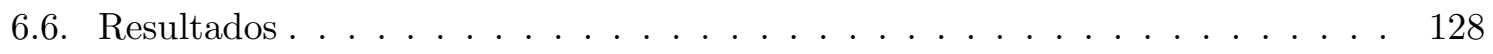

6.6.1. Distribución de Tamaños de los Troyanos de $L_{4}$. . . . . . . . . . . . . 129

6.6.2. Familias de Troyanos . . . . . . . . . . . . . . . . . 132

6.6.3. Tasas de Eyección desde el Punto de Equilibrio Joviano $L_{4} \quad \ldots \ldots$. . . 134

6.6.4. Polvo Troyano . . . . . . . . . . . . . . . . . . 137

6.6.5. Robustez de los Resultados . . . . . . . . . . . . . . . . . 141

7. Plutinos 143

7.1. Introducción . . . . . . . . . . . . . . . . . . . . . . . . 143

7.2. Poblaciones Iniciales ． . . . . . . . . . . . . . . . . . . . 144

7.3. Tratamiento Dinámico . . . . . . . . . . . . . . . . . . . . 147

7.4. Parámetros Colisionales . . . . . . . . . . . . . . . . . . 149

7.4.1. Probabilidad Intrínseca de Colisión y Velocidad de Impacto . . . . . . 149

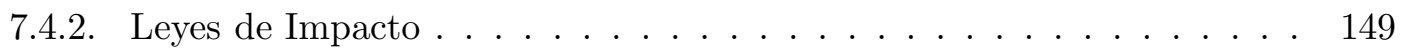

7.5. Modelo Dinámico y Colisional . . . . . . . . . . . . . . . . . . . . 151 
7.6. Resultados . . . . . . . . . . . . . . . . . . . 153

7.6.1. Distribución de Tamaños de los Plutinos . . . . . . . . . . . . . . 153

7.6.2. Familias de Plutinos . . . . . . . . . . . . . . . . . 155

7.6.3. Tasas de Eyección desde la Resonancia 3:2 con Neptuno . . . . . . . . 156

7.6.4. Sensibilidad al Número de Objetos del Tamaño de Plutón . . . . . . . 157

7.6.5. Robustez de los Resultados . . . . . . . . . . . . . . . . . 159 



\section{Índice de figuras}

1.1. Cráteres en el Sistema Solar interior . . . . . . . . . . . . . . 5

1.2. Cráteres en el Sistema Solar exterior . . . . . . . . . . . . . . . . . 7

2.1. G. Piazzi y Ceres . . . . . . . . . . . . . . . . . . . . . . 10

2.2. Puntos de equilibrio lagrangianos. . . . . . . . . . . . . . . . . 13

2.3. Estructura del Sistema Solar a gran escala: Cinturón de Kuiper y Nube de Oort 17

2.4. Sistema Solar actual . . . . . . . . . . . . . . . . . . . . 21

3.1. Distribución de los asteroides del Cinturón Principal, Cruzadores de Marte y NEOs con respecto al semieje mayor, excentricidad e inclinación . . . . . . . 28

3.2. Distribución de Troyanos jovianos de $L_{4}$ y $L_{5}$ respecto del semieje mayor, excentricidad e inclinación . . . . . . . . . . . . . . . . 33

3.3. Distribución de amplitudes de libración para los Troyanos jovianos de $L_{4} \quad$. . 34

3.4. Distribución de Centauros y objetos de la Región Transneptuniana con respecto al semieje mayor, excentricidad e inclinación . . . . . . . . . . . . 35

3.5. Estructura dinámica actual de la Región Transneptuniana. Figura realizada por Duncan et al. (1995), y adaptada por Morbidelli et al. (2008) . . . . . . . 37

3.6. Difusión caótica en la Región Transneptuniana. Figura extraída de Nesvorný \& Roig 2001 . . . . . . . . . . . . . . . . . . . . . . . 39

3.7. Principales resonancias internas en la conmensurabilidad 3:2 con Neptuno . . 41

3.8. Mapas de estabilidad de la resonancia $3: 2$ con Neptuno . . . . . . . . . . . . . 42

3.9. Clases Taxonómicas como función de la distancia heliocéntrica . . . . . . . . 52

3.10. Distribución de albedos de Troyanos jovianos y cometas . . . . . . . . . . 54

3.11. Clases taxonómicas entre Troyanos jovianos . . . . . . . . . . . . . 55

3.12. Distribución de colores de Centauros y objetos de la Región Transneptuniana, como función de los parámetros orbitales . . . . . . . . . . . . 57

3.13. Distribución de colores para Centauros, Plutinos y objetos del Disco Dispersado 59

3.14. Leyes de Impacto $Q_{\mathrm{S}}$ y $Q_{\mathrm{D}}$ obtenidas por diferentes autores . . . . . . . . . . 62

3.15. Formación de ondas en la distribución de tamaños . . . . . . . . . . . . 64

5.1. Histograma de asteroides en función del semieje mayor . . . . . . . . . . . . 76

5.2. Distribuciones de tamaños iniciales para el Cinturón Principal de asteroides . 78

5.3. Distribución de asteroides con respecto a los elementos propios semieje mayor y excentricidad, en la vecindad de la resonancia de movimientos medios 3:1 con Júpiter y de la resonancia secular $\nu_{6} \ldots \ldots \ldots$. . . . . . . . . . . . 79

5.4. Difusión en la vecindad de la resonancia de movimientos medios 3:1 con Júpiter 80 
5.5. Distribución de asteroides en la vecindad de la resonancia de movimientos medios $5: 2$ con Júpiter . . . . . . . . . . . . . . . . . 82

5.6. Ilustración del efecto Yarkovsky diurno y estacional . . . . . . . . . . . . . . 85

5.7. Variación del semieje mayor y remoción de asteroides producidas por el efecto Yarkovksy . . . . . . . . . . . . . . . . . . . 88

5.8. Geometría usada para calcular el factor de asimetría presente en el efecto Yarkovsky estacional . . . . . . . . . . . . . . . . .

5.9. Tasas de remoción de asteroides debidas al efecto Yarkovsky y las resonancias orbitales usadas en nuestras simulaciones . . . . . . . . . . . . .

5.10. Leyes de Impacto $Q_{\mathrm{S}}$ y $Q_{\mathrm{D}}$ para el estudio del Cinturón Principal y la población de NEAs . . . . . . . . . . . . . . . . . . . . .

5.11. Distribución de tamaños del Cinturón Principal de asteroides y distribución de magnitudes $H$ de la población de NEAs, después de $4.5 \times 10^{9}$ años . . . . . .

5.12. Distribución de tamaños del Cinturón Principal de asteroides después de $5 \times$

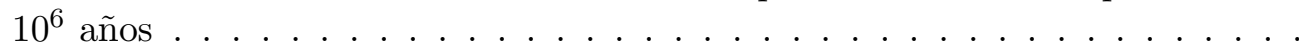

5.13. Remoción de asteroides por acción del efecto Yarkovsky y la inyección colisional dentro de las resonancias . . . . . . . . . . . . . . . . .

5.14. Histogramas del tiempo de exposición a los rayos cósmicos para condritas ordinarias .............................. 106

5.15. Tiempos de vida colisionales medios de los asteroides del Cinturón Principal .

5.16. Asteroide (4) Vesta . . . . . . . . . . . . . . . . . . .

5.17. Elementos propios $a$, e e $i$ para asteroides numerados, obtenidos de la base de datos de Knežević \& Milani (2000) . . . . . . . . . . . . . . . .

5.18. Distribución de velocidades para los fragmentos resultantes de una colisión .

5.19. Mezcla de material en el Cinturón Principal de asteroides . . . . . . . . . . .

6.1. Distribuciones de tamaños de los Troyanos jovianos de $L_{4}$ derivadas por Jewitt et al. (2000) y Yoshida \& Nakamura (2005). . . . . . . . . . . . . . . . . .

6.2. Distribución de tamaños inicial para la población de Troyanos jovianos de $L_{4}$.

6.3. Distribución de los Troyanos jovianos de $L_{4}$ con respecto al semieje mayor, excentricidad e inclinación.

6.4. Leyes de Impacto $Q_{\mathrm{S}}$ y $Q_{\mathrm{D}}$ para el estudio de los Troyanos jovianos de $L_{4} \ldots$

6.5. Distribuciones de tamaños finales de los Troyanos jovianos de $L_{4}$, después de $4.5 \times 10^{9}$ años, derivadas a partir de la población inicial PI1. . . . . . . . .

6.6. Distribuciones de tamaños finales de los Troyanos jovianos de $L_{4}$, después de $4.5 \times 10^{9}$ años, derivadas a partir de la población inicial PI2. . . . . . . . .

6.7. Dispersión de fragmentos colisionales con diámetros $\geq 0.1$ y $1 \mathrm{~km}$, como función del tiempo. . . . . . . . . . . . . . . . . . .

6.8. Tiempos de vida colisionales medios de los Troyanos jovianos de $L_{4} \ldots$. . . .

6.9. Tasas de eyección de fragmentos desde el enjambre Troyano joviano $L_{4}$. . . .

6.10. Distribución de los Troyanos eyectados desde el enjambre joviano $L_{4}$ en función del semieje mayor, excentricidad e inclinación . . . . . . . . . . . . . . . . 136

6.11. Luminosidad del polvo Troyano joviano de $L_{4} \ldots \ldots \ldots \ldots$. . . . . . . . 139

6.12. Evolución de los parámetros orbitales de los Troyanos jovianos de $L_{4}$. . . . . 142

7.1. Distribuciones de tamaños iniciales para la población de Plutinos . . . . . . . 146

7.2. Mapas de Densidad - Evolución Dinámica de Plutinos . . . . . . . . . . . . 148 
7.3. Leyes de impacto $Q_{\mathrm{S}}$ y $Q_{\mathrm{D}}$ para el estudio de Plutinos $\ldots \ldots \ldots$. . . . . . 150

7.4. Distribuciones de tamaños finales de Plutinos, después de $4.5 \times 10^{9}$ años de

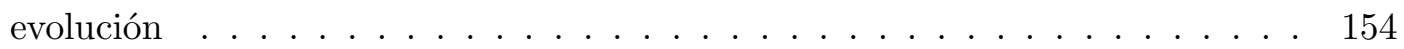

7.5. Tiempos de vida colisionales medios de Plutinos . . . . . . . . . . . . 155

7.6. Tasas de eyección de fragmentos colisionales desde la resonancia 3:2 con Neptuno156

7.7. Distribución de los fragmentos eyectados desde la resonancia 3:2 con Neptuno en función del semieje mayor, excentricidad e inclinación . . . . . . . . . . . . 158

7.8. Evolución de los parámetros orbitales de los Plutinos . . . . . . . . . . . . . 160 



\section{Índice de tablas}

3.1. Resultados dinámicos derivados por Bottke et al. (2002) para diferentes regiones fuentes de NEOs . . . . . . . . . . . . . . . . . . . . 32

3.2. Clases taxonómicas de asteroides definidas por Tholen et al. (1984) . . . . . . 50

5.1. Parámetros Térmicos de los Asteroides . . . . . . . . . . . . . . . . 87

5.2. Parámetros colisionales $\left\langle P_{\mathrm{i}}\right\rangle,\langle V\rangle, f_{\mathrm{ke}_{0}} \mathrm{y} \gamma$ para el estudio del Cinturón Principal y la población de NEAs . . . . . . . . . . . . . . . . . . . . 94

6.1. Valores medios de la velocidad de impacto $\langle V\rangle$ y la probabilidad intrínseca de colisión $\left\langle P_{\mathrm{i}}\right\rangle$ para los Troyanos jovianos . . . . . . . . . . . . .

6.2. Valores medios de la probabilidad intrínseca de colisión $\left\langle P_{\mathrm{i}}\right\rangle$ entre Troyanos jovianos de $L_{4}$ e Hildas y Cometas de la Familia de Júpiter . . . . . . . . . . 124 



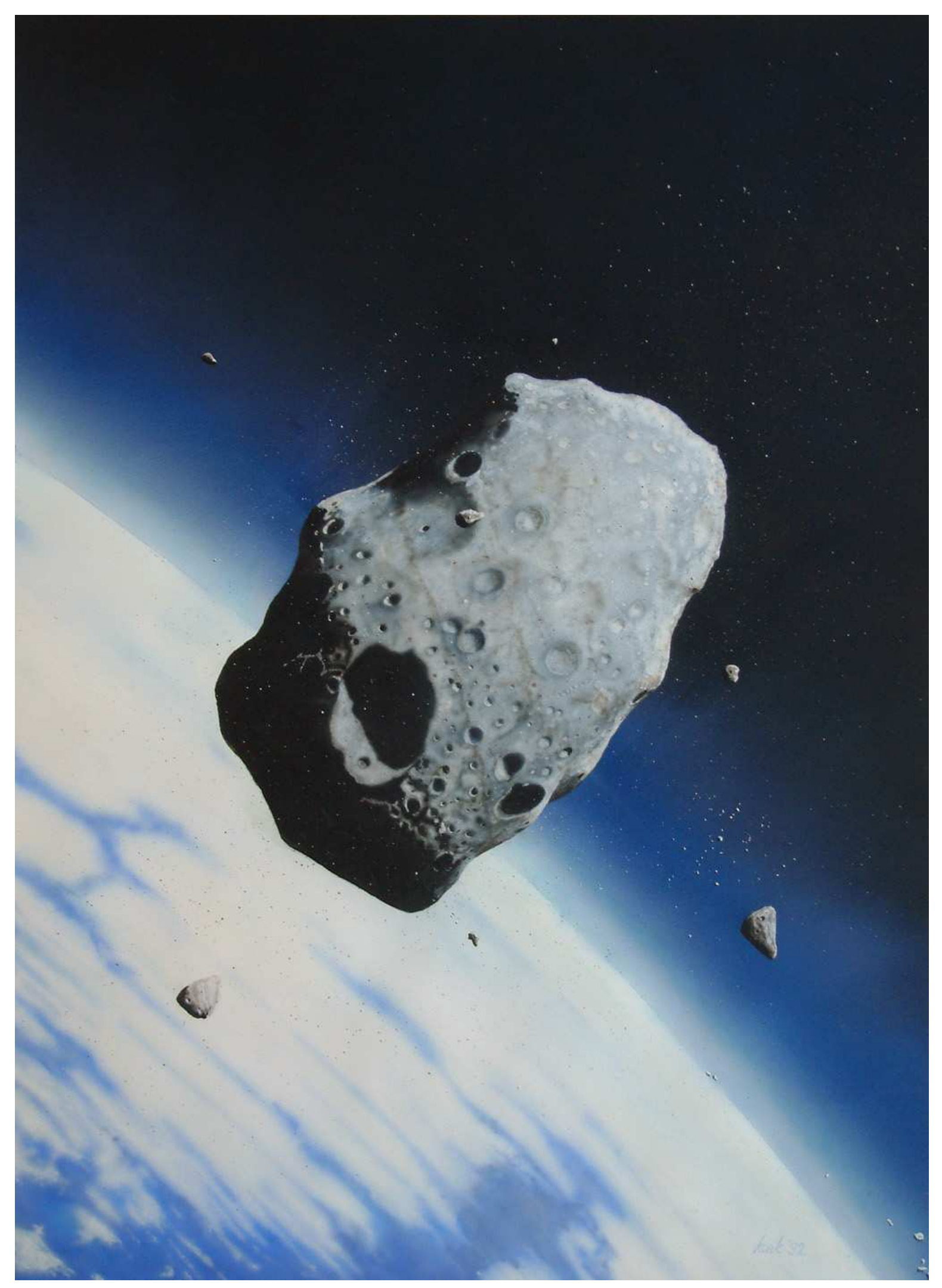





\section{Capítulo 1}

\section{Introducción}

El proceso de formación de nuestro Sistema Solar comenzó hace unos $4.5 \times 10^{9}$ años a partir del colapso gravitacional de una nube de gas y polvo interestelar. La evolución de esta estructura dio lugar al nacimiento de nuestro Sol y a la formación de la innumerable cantidad de objetos que orbitan alrededor del mismo.

Los miembros más representativos de nuestro Sistema Solar son los Planetas, cuyo número asciende a un total de ocho. De acuerdo a su distancia al Sol, estos objetos son agrupados en dos clases distintivas: los Planetas Interiores, cuyos representantes son Mercurio, Venus, Tierra y Marte, y los Planetas Exteriores, dados por Júpiter, Saturno, Urano y Neptuno. A excepción de Mercurio y Venus, todos los Planetas de nuestro Sistema Solar poseen al menos un satélite natural que los acompaña en su movimiento alrededor del Sol.

Desde el año 2006, los Planetas Enanos constituyen una nueva categoría de objetos del Sistema Solar. Si bien actualmente este grupo cuenta con Ceres, Plutón, Eris, Make Make y Haumea como sus cinco únicos representantes, se especula que muchos nuevos objetos serán incluidos dentro de esta categoría en un futuro próximo.

El mayor número de cuerpos de nuestro Sistema Solar lo proporcionan las poblaciones de Asteroides, Cometas, Centauros y Objetos de la Región Transneptuniana. El estudio de estas poblaciones de pequeños planetas resulta ser muy significativo ya que los mismos representan residuos del proceso de formación de nuestro Sistema Solar, y por ende nos permiten develar ciertos interrogantes asociados a aquellas etapas primitivas.

Los asteroides son cuerpos sólidos de estructura fundamentalmente rocosa. Si bien estos objetos se distribuyen en diferentes regiones, la gran mayoría de los mismos se ubica en el Cinturón Principal, el cual está localizado entre las órbitas de Marte y Júpiter. Otro grupo de interés está representado por los asteroides cercanos a la Tierra (Near-Earth Asteroids NEAs), los cuales resultan ser potenciales impactores sobre nuestro planeta. La fuente principal de estos objetos es el Cinturón Principal de asteroides, y la conexión entre ambas poblaciones se produce a través de mecanismos dinámicos asociados a las resonancias orbitales. Los Troyanos jovianos representan otra población distintiva de asteroides de nuestro Sistema Solar. Estos objetos libran alrededor de los puntos de equilibrio lagrangianos $L_{4}$ y $L_{5}$ de Júpiter, orbitando a $60^{\circ}$ por delante y por detrás del planeta.

Los cometas son cuerpos compuestos por una mezcla de polvo de silicatos, materiales orgánicos y hielos. Cuando un cometa se aproxima al Sol, su núcleo se calienta y los hielos se 
subliman, produciendo una nebulosidad característica, consistente de gas y granos de polvo. Históricamente, los cometas pueden ser clasificados en dos grandes grupos, de acuerdo a su período orbital $P$ : los Cometas de Largo Período, con $P>200$ años, y los Cometas de Corto Período, con $P<200$ años. Este último puede ser dividido a su vez en Cometas de la Familia de Júpiter, con $P<20$ años, y Cometas Tipo Halley, con $20<P<200$ años. Los Cometas de Largo Período y Tipo Halley tienen su fuente en la Nube de Oort, un amplio reservorio de cuerpos helados que rodea el Sistema Solar hasta distancias casi estelares. Por otra parte, la fuente principal de los Cometas de la Familia de Júpiter resulta ser la Región Transneptuniana.

La Región Transneptuniana está representada por un disco de planetesimales helados que se extiende hasta unas pocas centenas de unidades astronómicas. Los objetos transneptunianos presentan diferentes características dinámicas y pueden ser agrupados en cuatro clases distintivas: 1) los Objetos Resonantes, con movimientos medios conmensurables al del Neptuno, 2) los Objetos Clásicos, con semiejes mayores en el rango $42<a<48$ UA y bajas excentricidades, 3) los Objetos del Disco Dispersado, con distancias perihélicas en el rango 30 $<q<39$ UA y altas excentricidades e inclinaciones, y 4) los Objetos del Disco Dispersado Extendido, con distancias perihélicas $q \geq 39$ UA y semiejes mayores elevados. De estas cuatro clases dinámicas, el Disco Dispersado representa la fuente primaria de los Cometas de la Familia de Júpiter. Una población distintiva de la Región Transneptuniana está representada por los Plutinos, los cuales están asociados a la resonancia de movimientos medios 3:2 con Neptuno, ubicada a $\sim 39.5$ UA. Esta resonancia resulta ser la más densamente poblada de la Región Transneptuniana, y el nombre de sus miembros se debe a la analogía de sus órbitas con aquella de Plutón, el cual es su miembro más representativo.

Si los Cometas de la Familia de Júpiter provienen de la Región Transneptuniana, es evidente que debe existir un estado de transición, el cual transcurre mientras los cuerpos están ubicados entre las órbitas de Júpiter y Neptuno. En este estado, los objetos reciben el nombre de Centauros.

Los procesos colisionales han jugado un rol fundamental en la historia evolutiva de la mayoría de los cuerpos de nuestro Sistema Solar. En efecto, existen evidencias fehacientes que establecen que los eventos de impacto han sido responsables de diversas características observadas en algunos planetas y satélites naturales, así como también en las diferentes poblaciones de pequeños cuerpos de nuestro Sistema Solar.

Las Figuras 1.1 y 1.2 ilustran diversas estructuras de impacto observadas sobre diferentes cuerpos del Sistema Solar interior y exterior, respectivamente. En el Sistema Solar interior, la Luna (Figura 1.1a) y Mercurio (Figura 1.1b) son, quizás, los objetos que mejor exponen las evidencias de un pasado fuertemente activo desde un punto de vista colisional. Producto de una extensa inactividad geológica y la ausencia de una atmósfera significativa, estos objetos han logrado conservar sobre sus superficies una innumerable cantidad de estructuras de impacto, la mayoría de las cuales se formaron hace $\sim 3.8 \times 10^{9}$, en un evento conocido como Bombardeo Tardío (Hartmann et al. 2000). En menor medida, Marte también muestra signos de una fuerte actividad colisional. Particularmente, Fobos y Deimos, sus dos pequeñas lunas, presentan formas irregulares y superficies fuertemente craterizadas. La Figura 1.1c muestra un primer plano del cráter Stickney sobre Fobos, el cual representa la más relevante estructura de impacto sobre la superficie de esta luna marciana.

Nuestro planeta también ha sufrido las consecuencias de una fuerte actividad colisional 

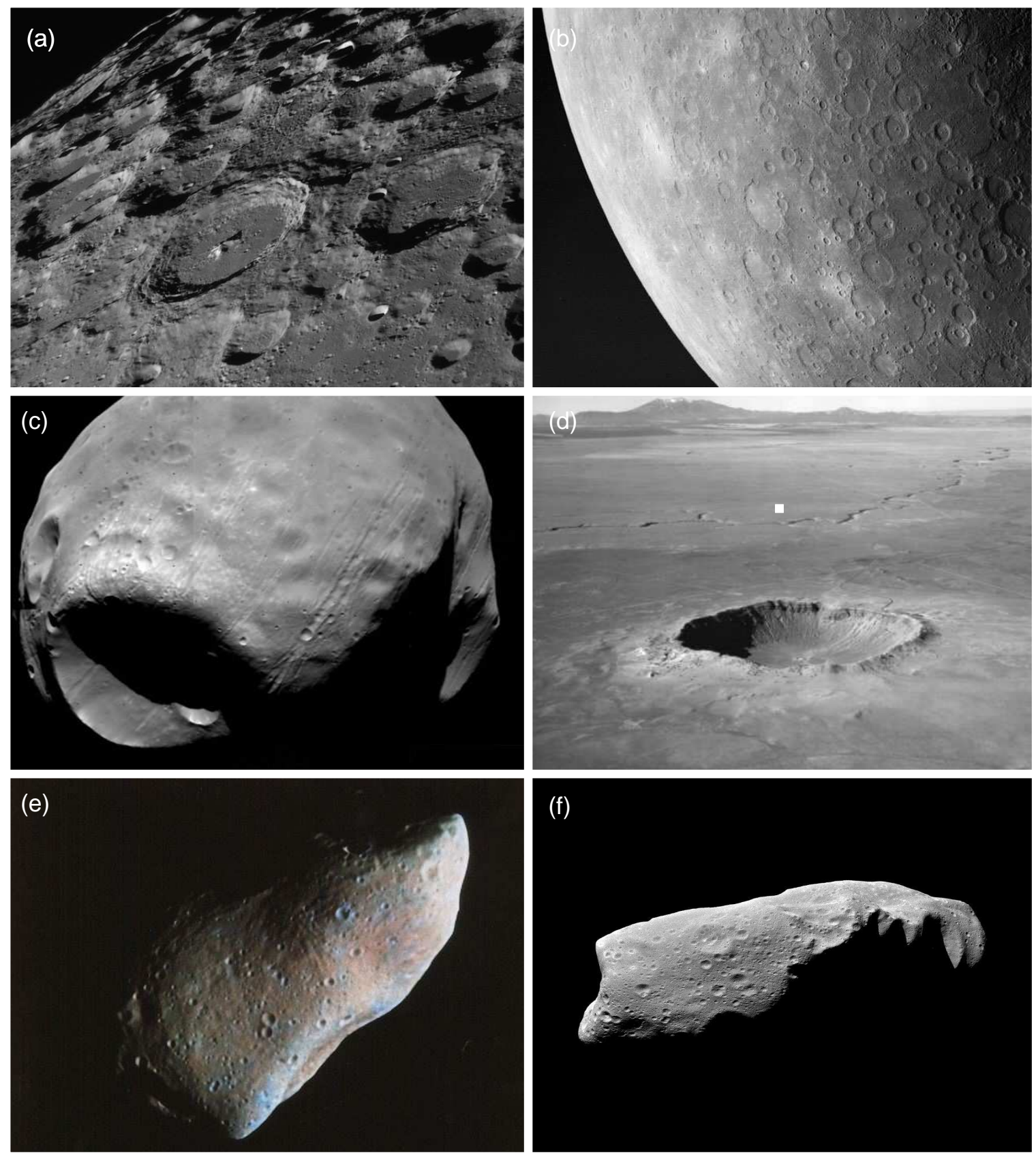

Figura 1.1. Evidencias de impactos en el Sistema Solar interior. a) La Luna (Créditos y derechos de autor: Wes Higgins). b) Mercurio (Créditos: MESSENGER, NASA, JHU APL, CIW). c) Fobos (Créditos: Viking Project, JPL, NASA; Mosaico de imágenes realizado por Edwin V. Bell II (NSSDC/Raytheon ITSS)) d) Cráter Barringer, en Arizona, Estados Unidos. e) Gaspra e f) Ida, asteroides del Cinturón Principal. 
a lo largo de toda su evolución. En efecto, más de 200 estructuras de impacto han sido confirmadas sobre la superficie de la Tierra, las cuales exponen un amplio rango de edades y tamaños. La Figura 1.1d nos muestra el Cráter Barringer, en Arizona, Estados Unidos. Con un diámetro de $\sim 1.2 \mathrm{~km}$, el mismo representa la estructura de impacto mejor conservada sobre la superficie de nuestro planeta. Se trata de un cráter relativamente joven, formado hace $\sim 5$ $\times 10^{4}$ años, como consecuencia del impacto de un asteroide con un diámetro en el rango de 10 a $50 \mathrm{~m}$ (Kring 2007). Los procesos colisionales han jugado un rol clave en la historia evolutiva de nuestro planeta. Existen evidencias precisas que vinculan la extinción de los dinosaurios con el impacto de un asteroide de $\sim 10 \mathrm{~km}$ de diámetro sobre la región de la Península de Yucatán, México, hace $\sim 6.5 \times 10^{7}$ años (Alvarez et al. 1980). Sin embargo, los impactos no sólo están asociados a etapas primitivas de la evolución de nuestro planeta. El 30 de Junio de 1908, un bólido se desintegró en las capas inferiores de la atmósfera, arrasando con los bosques de la región de Tunguska, Siberia, sobre un área de $2150 \mathrm{~km}^{2}$ alrededor del epicentro (Vasilyev 1998). Vale la pena destacar que existen diversas interpretaciones relacionadas a la composición y tamaño del objeto asociado a este evento (Turco et al. 1982, Sekanina 1983, Chyba et al. 1993).

Por otra parte, los procesos colisionales han jugado un rol fundamental en la historia evolutiva de los asteroides. En efecto, la enorme cantidad de meteoritos encontrados sobre la superficie de la Tierra son fragmentos resultantes de colisiones entre asteroides. Durante los primeros años de la década del 90, la nave espacial Galileo tomó las primeras fotografías de un asteroide, develando evidencias directas de una evolución colisional significativa. El 29 de Octubre de 1991, Galileo pasó a $1600 \mathrm{~km}$ del asteroide (951) Gaspra (Figura 1.1e). Las imágenes muestran que este objeto de $19 \mathrm{~km}$ de longitud presenta una forma irregular y una superficie poblada de cráteres. El 28 de Agosto de 1993, Galileo se aproximó al asteroide (243) Ida (Figura 1.1f), el cual posee una longitud de $52 \mathrm{~km}$. Las imágenes evidenciaron una gran cantidad de estructuras de impacto sobre su superficie y además descubrieron la existencia de un pequeño satélite natural de $1.6 \mathrm{~km}$ de longitud, llamado Dactyl. Al igual que Ida y Gaspra, la superficie de Dactyl está fuertemente craterizada.

En el Sistema Solar exterior, también existen evidencias claras de una actividad colisional significativa. Calisto, uno de los cuatro satélites galileanos, es el mejor ejemplo que ilustra la relevancia de los eventos de impacto. En efecto, Calisto es el objeto más fuertemente craterizado del Sistema Solar (Smith et al. 1979a, 1979b). La Figura 1.2a expone una visión global de las estructuras de impacto sobre la superficie de este satélite. En la misma se observa que la región central de los cráteres resulta ser brillante, lo cual podría ser debido al hielo excavado por los impactos. La Figura 1.2b muestra, de izquierda a derecha, los cráteres Chrysor y Aleyn sobre la superficie de Ganímedes, otro de los satélites galileanos. Ganímedes también muestra evidencias de una fuerte actividad colisional. Las Figuras 1.2c y 1.2d ilustran las superficies de Epimeteo y Febe, dos satélites naturales de Saturno. Las estructuras de impacto resultan ser más que evidentes.

La relevancia de los procesos colisionales y nuestro interés de analizar las características asociadas a las principales poblaciones de pequeños planetas del Sistema Solar, nos motivaron a desarrollar la investigación presentada en este trabajo.

El objetivo principal de esta Tesis es analizar la evolución colisional de las principales poblaciones de pequeños cuerpos de nuestro Sistema Solar, teniendo en cuenta los más importantes mecanismos dinámicos asociados a las mismas. Nuestro estudio incluye los asteroides 

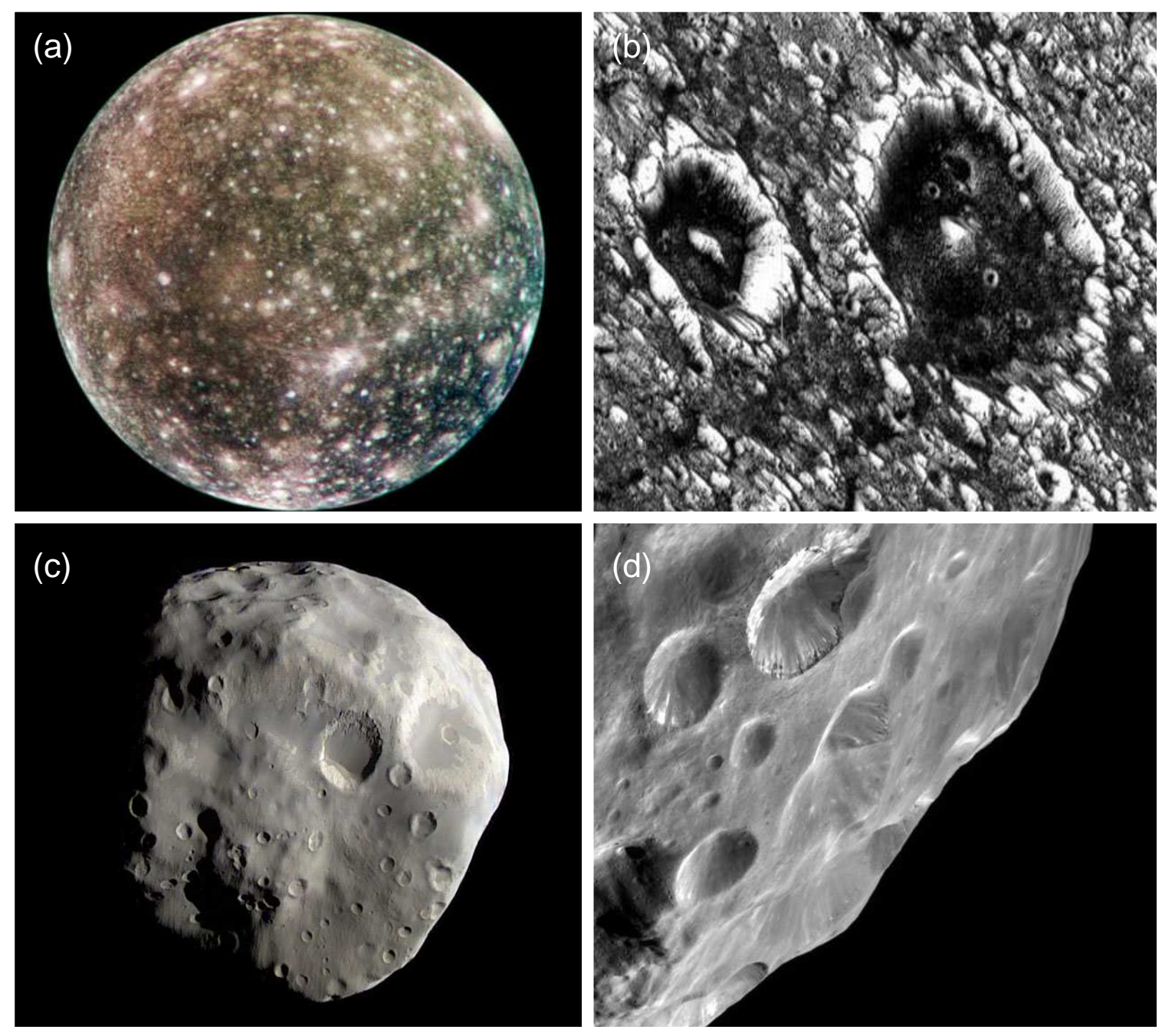

Figura 1.2. Evidencias de impacto en el Sistema Solar exterior. a) Calisto (Créditos: Galileo Project, Voyager Project, JPL, NASA) y b) Ganímedes (Créditos: Galileo Project, JPL, NASA), satélites de Júpiter. c) Epimeteo y d) Febe, satélites de Saturno (Créditos: Cassini Imaging Team, SSI, JPL, ESA, NASA).

del Cinturón Principal, los asteroides cercanos a la Tierra (NEAs), los Troyanos jovianos del punto de equilibrio $L_{4}$ y los Plutinos.

El estudio del Cinturón Principal de asteroides y la población de NEAs tiene como objetivo analizar la mezcla de material entre las diferentes regiones del cinturón de asteroides y evaluar el origen de los NEAs. Además, nuestro interés también está centrado en determinar los mecanismos más importantes que llevan a la inyección de material dentro de las resonancias orbitales del Cinturón Principal y dan lugar a la generación de los NEAs.

Por otra parte, nuestra investigación concerniente a los Troyanos jovianos de $L_{4}$ y los Plutinos tiene como motivación esencial analizar algún tipo de relación entre estos objetos y la población cometaria. 



\section{Capítulo 2}

\section{Perspectiva Histórica}

En este capítulo resumimos algunos de los sucesos más relevantes que llevaron al descubrimiento de las diferentes poblaciones de pequeños planetas del Sistema Solar ${ }^{1}$.

\section{1. $\quad$ El Descubrimiento de Ceres y el Origen de los Asteroides}

A través de los siglos, la existencia de un amplio gap entre las órbitas de Marte y Júpiter resultó ser un tema de debate general. El primero en sugerir la existencia de un planeta entre Marte y Júpiter fue el astrónomo alemán Johannes Kepler en su Mysterium Cosmographicum (1596). Posteriormente, muchos pensadores tales como I. Newton, E. Kant, J. H. Lambert, D. Gregory, W. Whiston y C. Wolff discutieron diferentes explicaciones concernientes a la existencia de dicho gap. En 1766, el astrónomo alemán Johann Daniel Titius publicó una ley que relacionaba las distancias medias de los planetas al Sol y sugería que entre Marte y Júpiter había un espacio vacío en el cual podría encontrarse un planeta aún no observado. Dicha relación llamó la atención de Johann Elert Bode, un astrónomo alemán que posteriormente llegaría a ser director del Observatorio de Berlín. Debido al interés de J. E. Bode, la relación de J. D. Titius asumió una gran importancia entre los astrónomos de la época. El descubrimiento de Urano en 1781, cuya órbita era bien ajustada por la ley de Titius, resultó una confirmación observacional de dicha relación y reforzó la creencia de la existencia de un planeta entre Marte y Júpiter.

Uno de los más interesados en la búsqueda de este "planeta perdido" fue el Barón Franz Xaver von Zach, un astrónomo de Gotha, director del Observatorio Seeberg. Para cumplir con este propósito, F. X. von Zach decidió organizar, junto con F. von Ende, W. Olbers, J. Gildemeister, J. H. Schroeter y K. L. Harding, una sociedad científica cuyo objetivo fue estudiar la región del zodíaco completa con la esperanza de detectar algún objeto nuevo. Dicha sociedad quedó establecida el 20 de Septiembre de 1800 y tomó el nombre de Vereinigte Astronomische Gesellschaft, usualmente conocida como "Sociedad Lilienthal". F. X. von Zach, junto con los otros 5 socios fundadores, dividieron el zodíaco en 24 zonas de $15^{\circ}$ de longitud y $\pm 7^{\circ}-8^{\circ}$ de latitud, y decidieron asignar cada una de estas regiones a un dado astrónomo. Para cumplir con este propósito, ellos requirieron la colaboración de otros 18 astrónomos.

\footnotetext{
${ }^{1}$ El material de este capítulo fue recopilado fundamentalmente de los escritos de Foderà Serio et al. (2002) y Davies et al. (2008)
} 


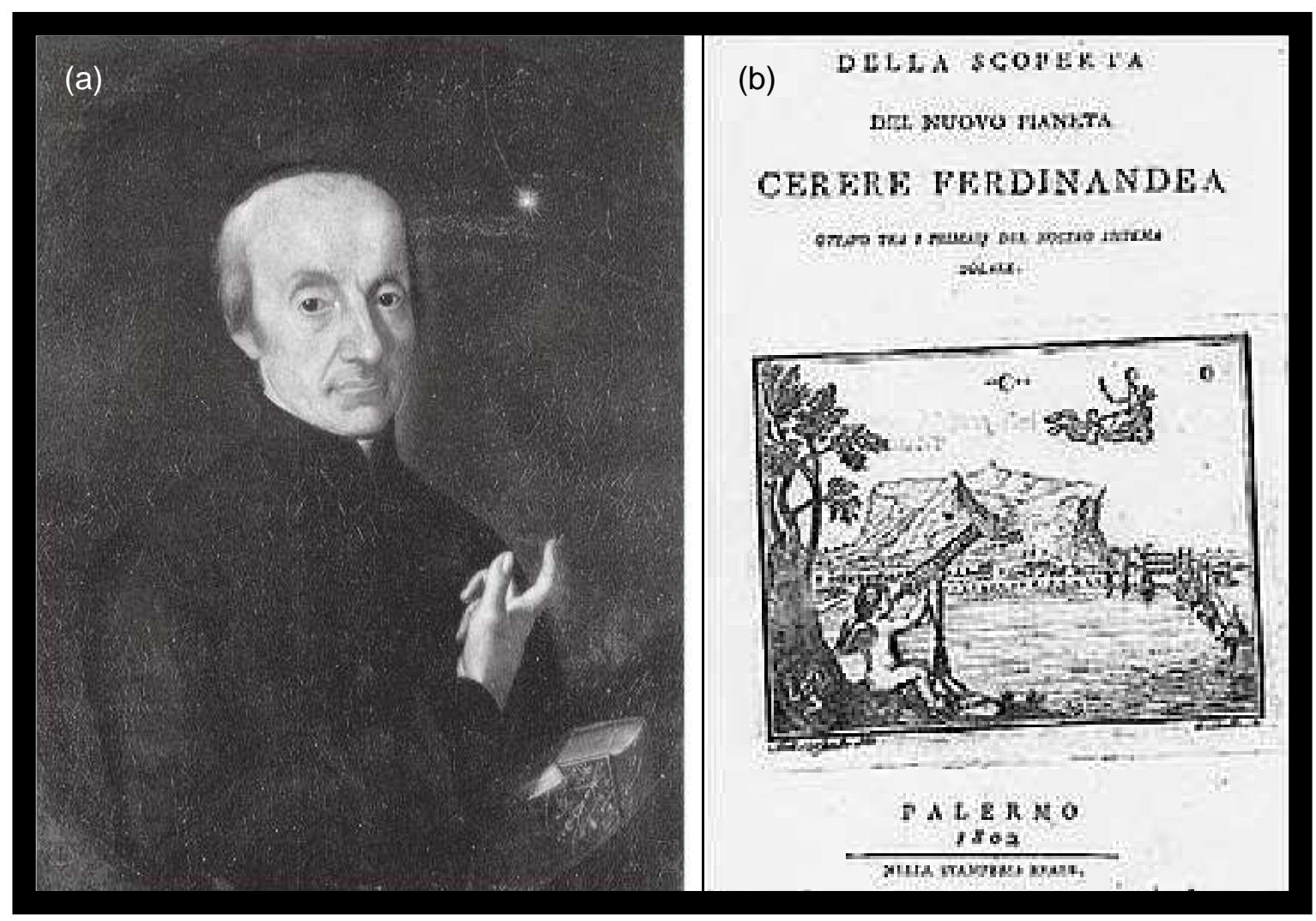

Figura 2.1. a) G. Piazzi y Ceres. b) Libro de G. Piazzi titulado Della scoperta del nuovo planeta Cerere Ferdinandea.

Los 24 miembros de la Sociedad Lilienthal finalmente fueron J. E. Bode (Berlín), J. S. G. Huth (Frankfurt/Oder), G. S. Klügel (Halle), J. A. Koch (Danzig), J. F. Wurm (Blauebeuren), F. von Ende (Celle), J. Gildemeister (Bremen), K. L. Harding (Lilienthal), W. Olbers (Bremen), J. H. Schroeter (Lilienthal), F. X. von Zach (Gotha), J. T. Bürg (Viena), T. Bugge (Copenhague), D. Melanderhielm (Estocolmo), J. Svanberg (Uppsala), F. T. Schubert (San Petesburgo), J. C. Burckhardt (París), P. F. A. Mechain (París), C. Thulis (Marsella), N. Maskelyne (Greenwich), W. Herschel (Slough), B. Oriani (Milán) y G. Piazzi (Palermo). Sin embargo, no todos estos astrónomos participaron activamente en los planes de la sociedad científica. Es más, muchos de ellos ni siquiera recibieron una invitación formal. Uno de estos fue el astrónomo Giuseppe Piazzi del Observatorio de Palermo. Sin embargo, la historia tendría preparado para él un lugar de relevancia.

Gioacchino Giuseppe Maria Ubaldo Nicolò Piazzi nació en Ponte, Valtelina, Italia, el 16 de Julio de 1746. Con sólo 19 años, G. Piazzi se unió a la Orden religiosa de los Teatinos. Entre 1770 y 1780, él enseñó filosofía y matemáticas en diferentes ciudades de Italia, tales como Roma, Génova y Ravena. En 1781, G. Piazzi fue nombrado Director de Matemáticas en la "Accademia dei Regi Studi" de Palermo la cual, en 1806, tomaría formalmente el nombre de Universidad de Palermo. En 1787, G. Piazzi fue nombrado Director de Astronomía aunque él, ni siquiera, era un astrónomo amateur. Sin embargo, en sólo unos pocos meses, él se convertiría en uno de los más respetados y calificados astrónomos de su época. En Marzo de 1787, G. Piazzi tomó la responsabilidad de construir un nuevo Observatorio en Palermo. 
Luego de recorrer los principales centros astronómicos de París y Londres, G. Piazzi retornó a Palermo y culminó la construcción del Observatorio en la cima de la torre de Santa Ninfa en el Palacio Real, en el año 1790. Allí, G. Piazzi contó con el telescopio más grande del siglo XVIII, alcanzando una precisión muy superior a la de los instrumentos existentes de la época.

Sin haber recibido una invitación formal de F. X. von Zach para participar de los planes de la Sociedad Lilienthal, G. Piazzi trabajó de manera independiente, centrando su programa científico en la medición precisa de posiciones estelares. A las 8:00 PM del 1 de Enero de 1801, G. Piazzi observó lo que él mismo calificó como una diminuta estrella de magnitud 8 en la región de Tauro (Piazzi 1801). G. Piazzi midió su posición, la reobservó la noche siguiente y encontró que la misma se había movido. Inicialmente él pensó que había cometido un error en sus cálculos, pero unos días después, más precisamente el 4 de Enero, él mismo confirmó que el objeto en cuestión era una nueva "estrella" o posiblemente un "cometa" sin una "nebulosidad apreciable". Tal como era costumbre en aquel tiempo, G. Piazzi informó a la prensa sobre su descubrimiento ese mismo día, y además envió cartas particulares con datos de sus observaciones a B. Oriani, J. E. Bode y J. Lalande. Inmediatamente después de recibir la carta de G. Piazzi, J. E. Bode creyó que el objeto en cuestión podía ser el famoso "planeta perdido". Motivado por esta idea, J. E. Bode anunció a la prensa el descubrimiento del nuevo planeta en Hamburgo, Jena y Berlín, bautizando al objeto con el nombre de "Juno". F. X. von Zach estaba en favor del nombre "Hera", el cual había sido propuesto por el Duque Ernesto de Sajonia-Gotha 15 años antes del descubrimiento del objeto, y fue el primero ampliamente aceptado, al menos en Alemania. G. Piazzi, quien había llamado a su objeto Ceres Ferdinandea en honor de la diosa patrona de Sicilia y al Rey Ferdinand de Borbón, estuvo en desacuerdo con los nombres propuestos por J. E. Bode y F. X. von Zach. G. Piazzi no ocultó su malestar, manifestando que por haber sido el descubridor del objeto, él tenía la prioridad en la elección del nombre. Finalmente, el nombre "Ceres" fue aquel aceptado por la comunidad astronómica, dado que, según F. X. von Zach, "Ceres Ferdinandea" era demasiado largo.

El 28 de Marzo de 1802, Wilhelm Olbers se encontraba en Bremen, Alemania, cuando detectó un diminuto objeto de magnitud 7 mientras observaba las pequeñas estrellas en el cúmulo de Virgo, con el objetivo de poder establecer más fácilmente la posición de Ceres. Dado que W. Olbers estaba absolutamente seguro de que dicho objeto no se encontraba allí dos meses atrás cuando había realizado sus primeras observaciones, él decidió tomar su posición y continuó observándolo durante dos horas. Sorprendentemente, W. Olbers encontró que el objeto en cuestión había cambiado su posición en ese pequeño intervalo, y luego de dos días de trabajo, él estaba seguro de que había detectado un nuevo planeta con características similares a las de Ceres, pero con una órbita más excéntrica e inclinada. W. Olbers bautizó a este objeto con el nombre de Palas e informó sobre su descubrimiento a F. X. von Zach y al resto de la comunidad astronómica. La mayoría de los astrónomos recibieron la noticia sin manifestar ningún tipo de sorpresa, ya que la idea que establecía que muchos otros nuevos cuerpos podrían existir entre las órbitas de Marte y Júpiter, e incluso entre los otros planetas, comenzaba a tener un consenso generalizado. El 6 de Mayo de 1802, William Herschel presentó a la Sociedad Real una memoria en la cual se manifestaba en favor de que los descubrimientos de G. Piazzi y W. Olbers habían puesto en evidencia la existencia de una nueva clase de objetos celestes en el Sistema Solar. W. Herschel asignó a estos nuevos cuerpos la denominación de asteroides debido a que sus apariencias eran similares a la de una estrella. La comunidad astronómica no adoptó las ideas de W. Herschel de manera inmediata; en efecto, el término 
asteroide llegó a ser ampliamente aceptado recién a principios del siglo XX.

En el mes de Junio de 1802, W. Olbers abordó el problema de reconciliar el descubrimiento de Palas con la ley de Titius-Bode sobre las distancias planetarias. Teniendo en cuenta que las distancias medias desde el Sol de Ceres y Palas eran bastante similares, W. Olbers sugirió que tales cuerpos eran residuos de un planeta que se había formado entre las órbitas de Marte y Júpiter y luego se había fragmentado debido a la acción de sus fuerzas internas o como consecuencia de una colisión con algún cometa. La teoría de W. Olbers parecía razonable y fue aceptada por muchos astrónomos, quienes razonaron que si una explosión catastrófica generó tales fragmentos, sus órbitas debían intersectarse en el lugar de la explosión y sobre el lado opuesto del Sol. A partir de esto, los astrónomos comenzaron a observar en las regiones de Cetus y Virgo, donde las órbitas de Ceres y Palas se intersectaban. Fue así que, el 1 de Septiembre de 1804, K. L. Harding, en Lilienthal, descubrió un nuevo pequeño planeta al cual bautizó como Juno.

Inmediatamente después de este descubrimiento, Hofrath Huth envió una carta a J. E. Bode ofreciendo una teoría diferente para el origen de Ceres, Palas y Juno. En efecto. H. Huth indicó que, entre las órbitas de Marte y Júpiter, la masa se coaguló en muchas pequeñas esferas, casi todas de las mismas dimensiones, al mismo tiempo en el cual se produjo la formación de los planetas en el Sistema Solar. Con este argumento, H. Huth sugirió que Juno no debía ser el último de los cuerpos descubiertos entre las órbitas de Marte y Júpiter. En efecto, el 29 de Marzo de 1807, W. Olbers observó en las mismas regiones del cielo donde Ceres, Palas y Juno habían sido descubiertos, y encontró su segundo asteroide, al cual C. F. Gauss bautizó como Vesta. Por casi 40 años los astrónomos no pudieron detectar ningún nuevo asteroide, por lo cual la comunidad científica convivió con la idea de la existencia de sólo cuatro de tales objetos en el Sistema Solar. Sin embargo, después de 15 años de duro trabajo, K. L. Hencke encontró el quinto asteroide en 1845, el cual recibió el nombre de Astraea.

A medida que los asteroides comenzaron a ser descubiertos en todas partes del cielo, la teoría de Olbers sobre el origen de tales objetos comenzó a ser ampliamente cuestionada. En efecto, en 1857, cuando alrededor de 50 asteroides habían sido descubiertos, el astrónomo francés François Arago sugirió que las órbitas de tales objetos eran inconsistentes con la hipótesis de Olbers, la cual indicaba que las mismas debían intersectarse en un mismo punto. En los años siguientes, la teoría de Olbers fue perdiendo el consenso general con el cual contaba desde sus inicios, y muchos astrónomos comenzaron a analizar diferentes escenarios para explicar el origen de los asteroides. Uno de ellos, Daniel Kirkwood, sugirió que los asteroides se originaron a partir de un anillo de material en el cual no se pudo formar ningún planeta de mayor tamaño, debido a las perturbaciones ejercidas por Júpiter (Kirkwood 1867). La idea propuesta por D. Kirkwood representa la teoría más ampliamente aceptada por los astrónomos modernos, concerniente al origen de los asteroides.

\subsection{Los Troyanos en el Sistema Solar}

En el año 1772, Joseph-Louis Lagrange demostró la existencia de cinco puntos de equilibrio en el problema restringido de tres cuerpos, en el cual un objeto de masa despreciable se mueve bajo la acción gravitatoria de dos cuerpos de mayor masa (Lagrange 1772). Tres de estos puntos, $\mathrm{L}_{1}, \mathrm{~L}_{2}$ y $\mathrm{L}_{3}$ se ubican sobre la línea que une a las dos masas principales y resultan ser inestables a pequeñas perturbaciones. Cada uno de los puntos restantes, $\mathrm{L}_{4} \mathrm{y} \mathrm{L}_{5}$, se ubican 


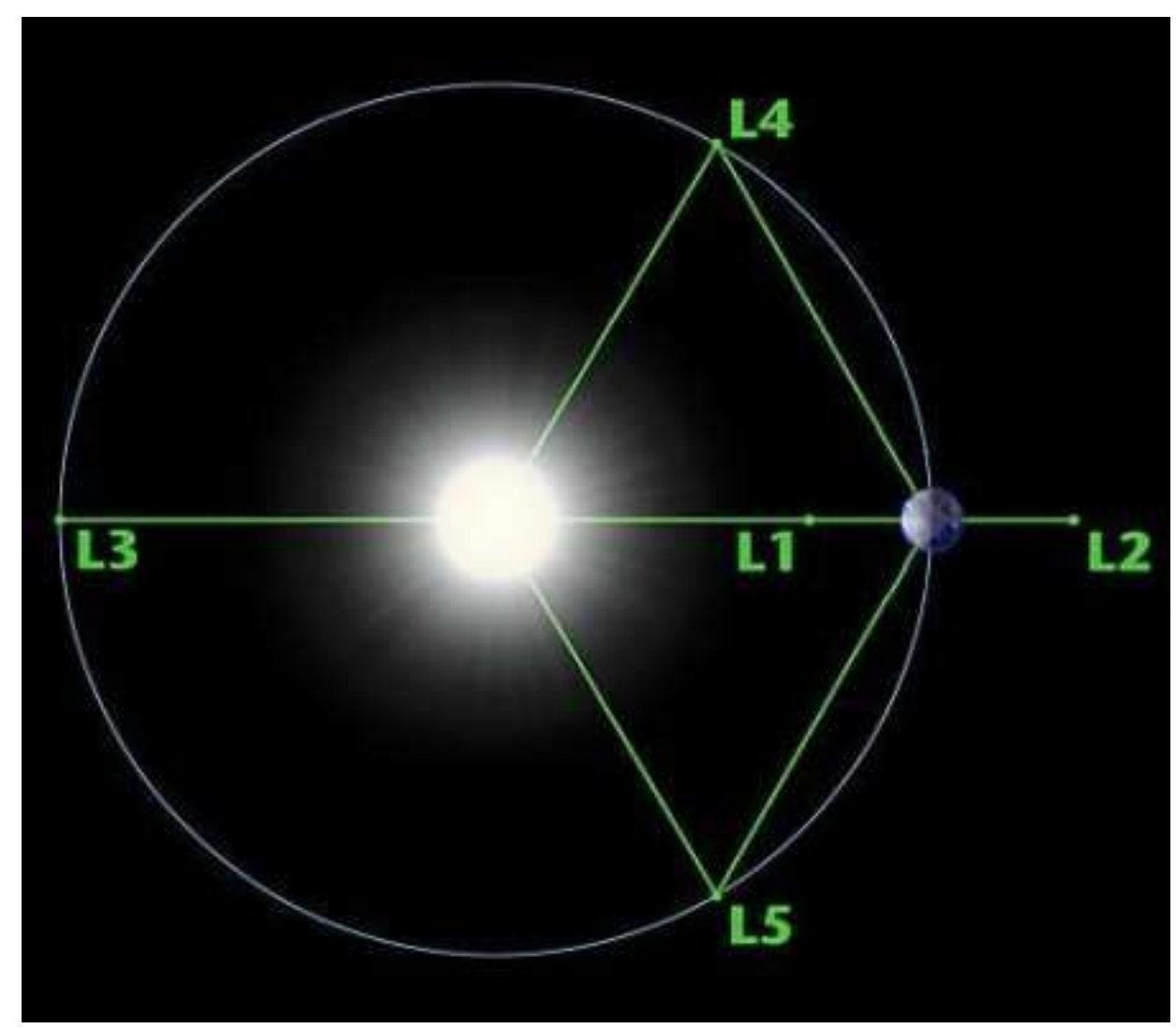

Figura 2.2. Puntos de equilibrio lagrangianos del problema restringido de tres cuerpos. $\mathrm{L}_{1}$, $\mathrm{L}_{2}$ y $\mathrm{L}_{3}$ resultan ser inestables a pequeñas perturbaciones. Por otra parte, $\mathrm{L}_{4} \mathrm{y} \mathrm{L}_{5}$ dan lugar a un movimiento estable.

en los vértices de un triángulo equilátero cuya base está dada por la separación entre las dos masas primarias. Una característica importante es que el movimiento estable es posible alrededor de tales puntos (Figura 2.2).

Los objetos localizados en los puntos lagrangianos $\mathrm{L}_{4}$ y $\mathrm{L}_{5}$ de la órbita de un planeta reciben el nombre de Troyanos. El grupo más numeroso de Troyanos conocido es aquel asociado a la órbita de Júpiter. En 1906, Max Wolf observó el primer Troyano joviano librando alrededor del punto $\mathrm{L}_{4}$, mientras que un segundo cuerpo fue encontrado en $\mathrm{L}_{5}$ por August Kopff el mismo año. Designados como (588) Achilles y (617) Patroclus, respectivamente, estos objetos representaron una confirmación observacional a los estudios de Lagrange realizados más de un siglo atrás. Hasta la fecha se han observado 2912 Troyanos en la órbita de Júpiter, de los cuales 1632 están asociados al punto de equilibrio $\mathrm{L}_{4}$ y 1280 a $\mathrm{L}_{5}$.

Más allá de aquellos asociados a Júpiter, objetos Troyanos han sido observados también en las órbitas de Marte y Neptuno. La identificación de los Troyanos de Marte es aún una materia de debate. En efecto, tal como lo indican Scholl et al. (2005), si bien diversos cuerpos han sido identificados como posibles Troyanos de Marte, sólo cuatro objetos han sido confirmados como tales. Por otra parte, si bien los estudios desarrollados por Sheppard \& Trujillo (2006) sugieren que la población de Troyanos de Neptuno debe ser 20 veces mayor que la de los Troyanos de Júpiter, sólo seis de tales objetos han sido descubiertos hasta aquí. 
Los estudios observacionales no han sido capaces de detectar objetos Troyanos en las órbitas de ninguno de los otros planetas del Sistema Solar. Destacamos que aún no existe un consenso generalizado que establece si esto es debido a cuestiones de inestabilidad dinámica en las órbitas de dichos planetas o a dificultades observacionales.

\subsection{La Región Transneptuniana}

Poco tiempo después de producirse el descubrimiento de Urano en 1781, diferentes astrónomos comenzaron a realizar determinaciones precisas de su órbita. Sin embargo, tales estimaciones permitieron observar que el movimiento de Urano no se correspondía precisamente con la órbita calculada. La mayoría de los astrónomos sugirieron que las perturbaciones ejercidas sobre Urano por parte del resto de los planetas afectaban levemente su movimiento, dando lugar a las discrepancias mencionadas entre la posición teórica y observada. Diversos especialistas calcularon de modo preciso los efectos de tales perturbaciones, encontrando que el camino descripto por Urano en su movimiento alrededor del Sol seguía sin ser absolutamente consistente con la órbita calculada. A partir de estos resultados, los astrónomos de la época sugirieron que más allá de Urano, debía existir un planeta desconocido el cual sería responsable del movimiento peculiar de Urano.

Entre 1841 y 1845, John Couch Adams, un joven estudiante de matemáticas de la Universidad de Cambridge, analizó el movimiento de Urano y computó las perturbaciones necesarias para compatibilizar las predicciones teóricas con las observaciones. A partir de este estudio, J. C. Adams calculó en qué región del cielo debía encontrarse aquel planeta desconocido, responsable de tales perturbaciones, aunque no logró que la comunidad astronómica pusiera atención en sus investigaciones.

Simultáneamente, y de manera independiente, Urbain LeVerrier, un joven astrónomo francés, también estudió el movimiento peculiar de Urano, llegando a las mismas conclusiones que J. C. Adams, concernientes a la posición del planeta desconocido. A partir de estos resultados, U. Leverrier contactó al astrónomo Johann Gottfried Galle, residente en Berlín, para darle las coordenadas precisas hacia las cuales apuntar su telescopio, con el fin de encontrar aquel objeto intrigante. Durante la primera noche de observación, el 23 de Septiembre de 1846, J. G. Galle y su ayudante, Heinrich Ludwing D'Arrest, observaron un objeto peculiar que no figuraba en la carta estelar de aquella región del cielo con la cual ellos contaban. Efectivamente, el "planeta desconocido", responsable de las irregularidades en el movimiento de Urano, había sido encontrado. Estos resultados significaron una confirmación observacional de las predicciones de J. C. Adams y U. Leverrier, quienes en la actualidad se dividen el mérito de este descubrimiento. Poco tiempo después, este cuerpo fue bautizado con el nombre de Neptuno, dios del mar, debido a su tonalidad verdosa.

\subsubsection{El Descubrimiento de Plutón}

Si bien fue ampliamente aceptado que Neptuno era el responsable de la mayor parte de las irregularidades observadas en la órbita de Urano, muchos astrónomos sugirieron que las perturbaciones ejercidas por un planeta desconocido, ubicado más allá de Neptuno, también debían ser investigadas. Uno de los más interesados en la búsqueda de este planeta fue Percival Lowell, un astrónomo estadounidense, fundador del Observatorio Lowell en 1894. Entre 1911 
y 1916, P. Lowell desarrolló sin éxito una exhaustiva búsqueda de este intrigante objeto en las regiones más exteriores del Sistema Solar. Después de la muerte de P. Lowell, el 16 de Noviembre de 1916, la búsqueda del planeta fue suspendida hasta 1929.

De aquí en más, el protagonista principal de esta historia comenzaría a ser Clyde W. Tombaugh, un joven perteneciente a una familia rural de Kansas, en los Estados Unidos. Si bien C. Tombaugh había sido un excelente estudiante en su paso por la escuela secundaria, al igual que el resto de la gente joven perteneciente a familias de granjeros, tenía pocas esperanzas de poder acceder a una educación universitaria. Sin embargo, desde su juventud, C. Tombaugh había sentido un gran interés por la astronomía, a partir de lo cual llevó a cabo la construcción de una serie de telescopios de gran poder y calidad sobre la granja de la familia. En Junio de 1928, C. Tombaugh hizo uso de su telescopio reflector de 9 pulgadas y tomó fotografías de las superficies de Marte y Júpiter, las cuales decidió enviar al Observatorio Lowell. El Dr. V. M. Slipher, Director del Observatorio, quedó impresionado con la calidad de las imágenes e inmediatamente envió una respuesta a C. Tombaugh, invitándolo a unirse al grupo de astrónomos del lugar, por un período de prueba de tres meses.

C. Tombaugh arribó al Observatorio Lowell el 15 de Enero de 1929. Allí, él se familiarizó con el uso del nuevo telescopio de 13 pulgadas, el cual había sido construido con el propósito principal de encontrar el planeta predicho por P. Lowell. El Dr. V. M. Slipher, Director del Observatorio, quería ser el primero en encontrar el noveno planeta del Sistema Solar. Sin embargo, el año 1929 resultó ser más que frustrante en este aspecto, ya que su búsqueda no le proporcionó los resultados esperados.

Sin embargo, durante los primeros meses de 1930, la historia comenzaría a ser otra. A las cuatro de la tarde del 18 de Febrero de 1930, C. Tombaugh se encontraba estudiando una región de la constelación de Géminis, comparando observaciones realizadas entre las noches del 23 y 29 de Enero. En aquel análisis, C. Tombaugh encontró un objeto que cambiaba su posición 3.5 milímetros durante las seis noches de observación, lo cual indicaba que el mismo debía encontrarse a algo más de 10 UA más allá de Neptuno. Inmediatamente, C. Tombaugh se convenció de que el objeto encontrado era un nuevo planeta, el noveno de nuestro Sistema Solar.

Después del descubrimiento, Constance Lowell, viuda de Percival, quiso bautizar al nuevo planeta con el nombre "Lowell". Sin embargo, pronto cambió su parecer, y prefirió llamar a este objeto "Constance". Para evitar complicaciones en la elección del nombre, el Observatorio Lowell decidió llevar a cabo una votación entre sus miembros. El nombre elegido fue finalmente Plutón, un dios del mundo inferior, sugerido por una pequeña niña inglesa de 11 años.

Ernest C. Bower y Fred L. Whipple, estudiantes graduados de la Universidad de California en Berkeley, computaron la órbita de este nuevo objeto, a partir de observaciones obtenidas en el Observatorio Lick por F. W. Meyer. Ellos mostraron que este cuerpo se encontraba a 41 UA de la Tierra en una órbita inclinada en $17^{\circ}$ respecto del plano de la eclíptica. Ya que su excentricidad orbital estaba completamente indeterminada, la pertenencia de este objeto a la amplia región ubicada más allá de Neptuno era sólo una especulación.

El 14 de Abril de 1930, una semana después de la publicación de las conclusiones de E. C. Bower y F. L. Whipple, el Observatorio Lowell presentó sus resultados concernientes a las propiedades orbitales de este nuevo cuerpo. Los mismos estaban basados en tres observaciones espaciadas a intervalos de un mes. El reporte indicaba valores específicos de 0.909 para la excentricidad y más de 3000 años para el período orbital. A mediados de Mayo de 1930, el 
reconocimiento de imágenes de Plutón anteriores a su descubrimiento le permitieron a Andrew C. D. Crommelin concluir que este nuevo cuerpo tenía una excentricidad orbital levemente menor a 0.3 y un perihelio localizado dentro de la órbita de Neptuno. Estos resultados fueron confirmados durante las siguientes semanas, cuando nuevas imágenes de Plutón, previas a su descubrimiento, fueron encontradas.

\subsubsection{Contribuciones de Edgeworth y Kuiper}

Si bien muchas autoridades tales como Campbell (1916), Aitken (1926) y Leuschner (1927) especularon durante muchos años sobre la existencia de planetas transneptunianos y la distribución de pequeños cuerpos en el Sistema Solar exterior, el trabajo desarrollado por el astrónomo irlandés Kenneth E. Edgeworth $(1938,1943,1949)$ resulta ser la primera investigación cuantitativa concerniente a la posible existencia de un gran número de potenciales cometas en un anillo más allá de las órbitas de Neptuno y Plutón.

K. E. Edgeworth desarrolló una discusión sobre los efectos de las fuerzas viscosas y de mareas sobre la disipación del momento angular en un disco primordial de gas y pequeñas partículas, orbitando alrededor del Sol. Si el sistema fuese lo suficientemente denso como para causar que el mismo sea capaz de condensarse en diversas subregiones, estas se ligarían entre sí, dando lugar a la formación de los planetas. Sin embargo, más de allá de Neptuno y Plutón, la densidad del disco sería más baja y los procesos de condensación que llevaron a la formación de los planetas, tendrían un tiempo insuficiente para operar eficazmente y formar grandes cuerpos de tamaño planetario. En efecto, K. E. Edgeworth sugirió que debido al decrecimiento de la densidad en las partes más externas de la nebulosa solar y la menor velocidad de condensación en esta región, la tasa de crecimiento de los cuerpos individuales decrecería rápidamente con un incremento de la distancia heliocéntrica. A partir de esto, K. E. Edgeworth calculó que a grandes distancias, los procesos de condensación producirían una estructura con una enorme cantidad de pequeños objetos, la cual sobreviviría hasta los tiempos actuales. Él sugirió que estos cuerpos podrían llegar a ser visibles a modo de cometas observables si los mismos fuesen perturbados y luego inyectados en el Sistema Solar interior.

Por otra parte, K. E. Edgeworth realizó estimaciones del orden de magnitud del número y tamaño de los potenciales cometas más allá de Neptuno. Para $0.33 \mathrm{M}_{\oplus}$ de material en dicha región, K. E. Edgeworth calculó la existencia de $2 \times 10^{8}$ objetos con masas individuales de $2 \times 10^{-9} \mathrm{M}_{\oplus}$. Por otro lado, para $0.1 \mathrm{M}_{\oplus}$ de material en el anillo transneptuniano, K. E. Edgeworth estimó la existencia de $2 \times 10^{9}$ objetos con masas individuales de $5 \times 10^{-11} \mathrm{M}_{\oplus}$. Sus cálculos lo llevaron a sugerir que estos objetos deberían ser más chicos y más numerosos que los asteroides del Cinturón Principal. Además, K. E. Edgeworth estimó que el anillo de material transneptuniano debía extenderse entre las 65 y quizás hasta más allá de las 260 UA.

A partir de sus estudios, K. E. Edgeworth indicó que Neptuno representó el caso límite para la formación de un objeto de tamaño planetario en el Sistema Solar exterior. De acuerdo a su postura, las estimaciones de la masa asociada a la Región Transneptuniana indicaban que sería imposible formar un único objeto transneptuniano de tamaño planetario. Para K. E. Edgeworth, el estatus de Plutón estaba entre el de un planeta y un satélite escapado de Neptuno.

Una segunda contribución significativa concerniente al estudio del origen del Sistema Solar 


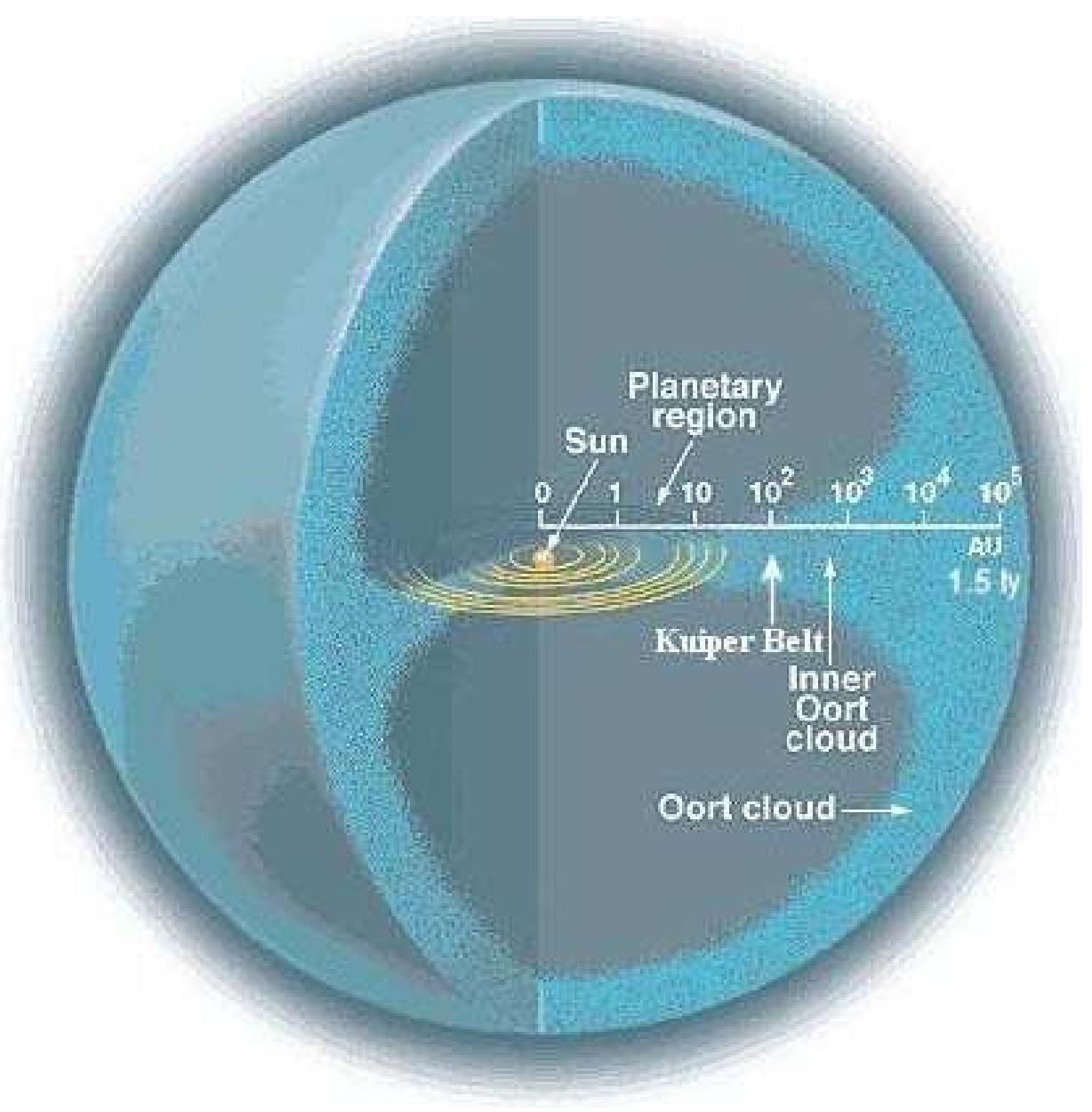

Figura 2.3. Estructura del Sistema Solar a gran escala. Por un lado, se observa la Nube de Oort como un reservorio esferoidal de cuerpos helados. Por otra parte, se ilustra el disco de planetesimales sugerido por K. E. Edgeworth y G. P. Kuiper más allá de la órbita de Neptuno.

fue dada por Gerard P. Kuiper (1951a, 1951b, 1956). G. P. Kuiper consideró la existencia de un cinturón de material más allá de la órbita de Neptuno, extendiéndose, aproximadamente, desde 38 hasta 50 UA. Él sugirió que las temperaturas en esta región fueron lo suficientemente bajas como para que el vapor de agua, el metano y el amoníaco se condensaran para formar "pequeños copos de nieve", y luego objetos de unas pocas decenas de centímetros. G. P. Kuiper explicó que estas "bolas de hielo" continuarían combinándose, incluso después de la disipación de la nebulosa solar, de modo que, sobre escalas de tiempo de $10^{9}$ años, el tamaño promedio de los objetos en la Región Transneptuniana sería de $1 \mathrm{~km}$, mientras que los más grandes tendrían tamaños superiores a los $100 \mathrm{~km}$. Si este cinturón de material tuviese una masa de $5 \times 10^{24} \mathrm{~kg}$, G. P. Kuiper sugirió que esto sería consistente con las estimaciones de Oort (1950), quien computó la existencia de $\sim 10^{11}$ miembros en un reservorio de cometas esferoidal con una masa total de $10^{24} \mathrm{~kg}$ (Figura 2.3) ${ }^{2}$.

Por otra parte, G. P. Kuiper indicó que los cometas probablemente no se han formado entre

\footnotetext{
${ }^{2}$ Este reservorio de cuerpos helados se conoce como Nube de Oort.
} 
Marte y Júpiter, tal como había especulado J. H. Oort. En efecto, G. P. Kuiper argumentó que, debido a las perturbaciones de Plutón, muchos de estos objetos son llevados hacia Neptuno, y luego, como consecuencia de otras perturbaciones planetarias, hacia el reservorio de cometas de Oort. Este mecanismo requería que Plutón tuviese una masa entre $0.1-1 \mathrm{M}_{\oplus}$, lo cual en aquellos tiempo fue ampliamente aceptado. G. P. Kuiper concluyó que los cometas que vemos hoy provienen de la Nube de Oort, lo cual justificaba la distribución isotrópica de las direcciones de los cometas conocidos en aquellos tiempos. Según lo sugerido por Oort, estos objetos serían removidos de dicho reservorio como consecuencia de las perturbaciones ejercidas por pasajes estelares. Además, G. P. Kuiper indicó que más allá del afelio de Plutón a 50 UA, donde las perturbaciones de este objeto resultan ser despreciables, podría aun existir un cinturón primordial de material residual y estar poblado por cometas.

\subsubsection{Contribuciones Modernas}

A principios de la década del 70, Everhart (1972) estudió el origen de los cometas de corto período. Everhart (1972) argumentó que repetidas interacciones con Júpiter de un conjunto de objetos sobre órbitas casi parabólicas, pueden producir cometas de corto período, siempre y cuando la inclinación inicial sea pequeña $\left(i \lesssim 9^{\circ}\right)$ y la distancia perihélica inicial esté cercana a la órbita de Júpiter $(4 \lesssim q \lesssim 6 \mathrm{UA}$ ). Además, él mostró que este mecanismo da lugar a una distribución de elementos orbitales asociada a los cometas de corto período que resulta ser consistente con las observaciones.

Sin embargo, de modo inmediato, Joss (1973) encontró una falencia significativa en este escenario. En efecto, él indicó que la eficiencia del proceso presentado por Everhart (1972) es demasiado baja como para reproducir el número observado de cometas de corto período a partir del flujo conocido de cometas casi parabólicos. Tiempo después, Everhart (1977) mostró que diversos cuerpos en órbitas casi parabólicas con perihelios tan grandes como la órbita de Neptuno, son dispersados gravitacionalmente por los planetas exteriores hacia la región de Júpiter, y luego, algunos de estos objetos llegan a ser cometas de corto período. En modelos convencionales de la Nube de Oort, la eficiencia de este proceso sigue siendo demasiado baja y no permite solucionar las falencias remarcadas por Joss (1973).

Un trabajo pionero concerniente al origen de los cometas de corto período es aquel desarrollado por Fernández (1980). Él mostró que si los cometas de corto período se hubiesen originado a partir de una población de objetos sobre órbitas casi parabólicas e isotrópicas, este proceso hubiese dado lugar a la remoción de más de $10^{12}$ cometas de largo período desde la Nube de Oort, sobre la edad del Sistema Solar. Sin embargo, esta cantidad resulta ser un orden de magnitud mayor que el número de miembros propuestos por Oort (1950) en este reservorio, lo cual parece ser excesivo. En vistas de esta dificultad, Fernández (1980) sugirió que el cinturón de material ubicado en la Región Transneptuniana, entre 35 y 50 UA, debía ser la fuente de los cometas de corto período. Además, Fernández (1980) puntualizó que este cinturón de cometas ubicado más allá de Neptuno no debe ser presentado como un substituto de la Nube de Oort, sino como un complemento. En efecto, mientras que dicho cinturón constituye la fuente de cometas de corto período, la Nube de Oort resulta ser la fuente principal de los cometas de largo período.

Algunos años más tarde, Duncan et al. (1988) utilizaron simulaciones numéricas para estudiar el origen de los cometas de corto período. Estos autores analizaron la evolución de 
órbitas cometarias debido a las perturbaciones gravitacionales de los planetas exteriores. Ellos concluyeron que, ya que la mayoría de los cometas de corto período tienen órbitas directas y de baja inclinación, los mismos no pueden ser generados a partir de la dispersión gravitacional desde un reservorio esférico, tal como la Nube de Oort. Sin embargo, Duncan et al. (1988) mostraron que la distribución de elementos orbitales de los cometas de corto período, obtenida a partir de una población de baja inclinación sobre órbitas que cruzan Neptuno, está en un excelente acuerdo con las observaciones. A partir de estos resultados, los autores corroboraron la sugerencia de Fernández (1980), concluyendo que un cinturón de cuerpos helados ubicado en la Región Transneptuniana debe ser la fuente de los cometas de corto período. Duncan et al. (1988) llamaron a esta fuente Cinturón de Kuiper, ya que al momento de realizar el trabajo, ellos no estaban familiarizados con el estudio de K. E. Edgeworth.

Con excepción de Plutón y Caronte, su mayor satélite natural, D. Jewitt y J. Luu descubrieron el primer objeto de la Región Transneptuniana en Septiembre de 1992, haciendo uso del telescopio de $2.2 \mathrm{~m}$ de la Universidad de Hawaii, en Mauna Kea. Designado como 1992 $\mathrm{QB}_{1}$, este objeto fue reportado el 14 de Septiembre en Jewitt \& Luu (1992) ${ }^{3}$. Este suceso representó una confirmación observacional de las predicciones teóricas descriptas anteriormente. Hasta la fecha, se han observado 1000 objetos transneptunianos, evidenciando una amplia diversidad de características dinámicas.

\subsection{El Descubrimiento de Eris (2003 UB313) y la Nueva De- finición de Planeta}

Desde el descubrimiento de Plutón en 1930 y por más de 70 años, la comunidad astronómica convivió con la idea de la existencia de un total de nueve planetas en nuestro Sistema Solar. Sin embargo, durante la década del 90, el descubrimiento de nuevos objetos más allá de la órbita de Neptuno, con características físicas y dinámicas similares a las de Plutón, puso una luz de alerta sobre la naturaleza y la clasificación de este diminuto planeta helado.

A fines del año 2003 tuvo lugar un suceso relevante que sería determinante en esta historia. El 21 de Octubre de ese año, M. E. Brown, C. A. Trujillo y D. L. Rabinowitz descubrieron el objeto transneptuniano 2003 UB313 haciendo uso del Telescopio Samuel Oschin de $1.2 \mathrm{~m}$ del Observatorio Palomar. Las primeras determinaciones orbitales permitieron inferir que este objeto es un miembro del Disco Dispersado de la Región Transneptuniana, con un semieje mayor $a=67.89 \pm 0.01 \mathrm{UA}$, una excentricidad $e=0.4378 \pm 0.0001$ y una inclinación $i$ $=43.993^{\circ} \pm 0.001^{\circ}$. El resultado más relevante obtenido por estos autores establece que 2003 UB313 posee un tamaño mayor al de Plutón (Brown et al. 2005b). Tiempo después, mediciones directas del tamaño de este objeto realizadas con el Telescopio Espacial Hubble permitieron confirmar este resultado (Brown et al. 2006). En efecto, este análisis asignó a 2003 UB313 un diámetro de $2400 \pm 100 \mathrm{~km}$, el cual es levemente superior al diámetro de Plutón de $2290 \mathrm{~km}$.

El descubrimiento de 2003 UB313 obligó a la comunidad astronómica a redefinir el concepto de "Planeta". En efecto, si Plutón continuaba siendo considerado un planeta, 2003 UB313 también debía serlo, así como también todos los potenciales objetos a ser descubiertos con tamaños comparables o incluso mayores a los de estos cuerpos.

\footnotetext{
${ }^{3}$ Los detalles de su descubrimiento pueden ser encontrados en Jewitt \& Luu (1993)
} 
La discusión sobre la nueva definición de planeta fue tratada en la XXVI Asamblea General de la Unión Astronómica Internacional (UAI), la cual se desarrolló desde el 14 al 25 de Agosto de 2006 en la ciudad de Praga, República Checa. La resolución final sobre la definición de planeta fue tomada el 24 de Agosto. Si bien la Asamblea General contó con 2412 participantes, sólo 424 miembros de la UAI emitieron su voto. A partir de lo discutido en esta Asamblea General, la UAI resolvió que, a excepción de los satélites, los objetos del Sistema Solar sean clasificados en tres categorías diferentes, de acuerdo a la forma siguiente:

1. Planeta: Un "Planeta" es un cuerpo celeste que

a) está en órbita alrededor del Sol,

b) tiene una forma aproximadamente esférica, y

c) ha limpiado la vecindad de su órbita.

2. Planeta Enano: Un "Planeta Enano" es un cuerpo celeste que

a) está en órbita alrededor del Sol,

b) tiene una forma aproximadamente esférica,

c) no ha limpiado la vecindad de su órbita,

d) nos es un satélite.

3. Pequeños Cuerpos del Sistema Solar: A excepción de los satélites, todos los otros objetos en órbita alrededor del Sol serán calificados como "Pequeños Cuerpos del Sistema Solar".

La decisión más resonante tomada por la UAI fue excluir a Plutón de la categoría de Planeta, reduciendo el número de tales cuerpos en el Sistema Solar a ocho. Con esto, Plutón fue incluido dentro de la categoría de Planeta Enano, junto con Ceres, hasta entonces el asteroide de mayor tamaño, y 2003 UB313, el objeto más grande conocido de la Región Transneptuniana. El 13 de Septiembre de 2006, el Comité Ejecutivo de la UAI aprobó el nombre de Eris para 2003 UB313. Eris es la diosa griega de la discordia y la contienda y según la mitología generó una disputa entre los dioses que llevó a la guerra de Troya. Dadas las posturas controversiales generadas a partir del descubrimiento de Eris (2003 UB313) concernientes a la definición de Planeta, creemos que el nombre elegido fue muy apropiado.

Durante el año 2008, la UAI designó a dos nuevos Planetas Enanos, aumentando a cinco el número de representantes de esta nueva categoría de cuerpos del Sistema Solar. En efecto, el 17 de Julio de 2008 la UAI decidió incluir al objeto 2005 FY9 dentro de la categoría de Planeta Enano, asignándole la denominación de Make Make. Este objeto, descubierto por M. E. Brown, C. A. Trujillo y D. L. Rabinowitz el 31 de Marzo de 2005, es un miembro del Disco Dispersado de la Región Transneptuniana, con un semieje mayor $a=45.7$ UA, una excentricidad $e=0.15$ y una inclinación $i=29^{\circ}$ (Brown et al. 2008). Mediciones realizadas con el Telescopio Espacial Spitzer sugieren que Make Make (2005 FY9) posee un diámetro de $1500 \pm 300 \mathrm{~km}$ (Stansberry et al. 2008). "Make Make" es el creador de la humanidad y el dios de la fertilidad en la mitología de la Isla de Pascua, en el Pacífico Sur. Este nombre fue propuesto por el equipo de trabajo que llevó a cabo su descubrimiento. Si bien M. E. Brown no estaba familiarizado con la mitología de dicha isla, la elección del dios de la fertilidad no fue casual, sino motivada por el embarazo de su esposa. El 17 de Septiembre de 2008, la UAI decidió clasificar al objeto 2003 EL61 dentro de la categoría de Planeta Enano y le 


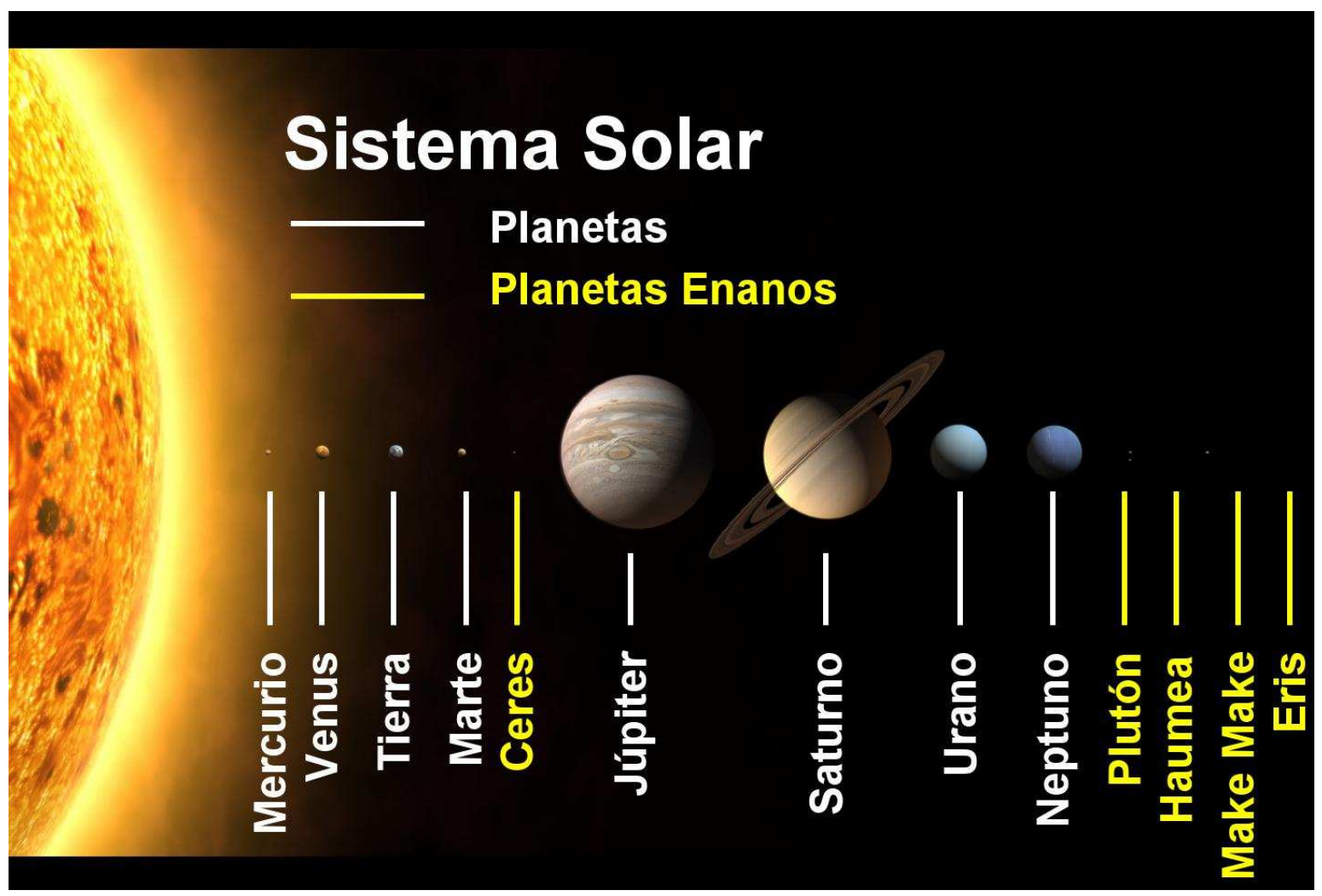

Figura 2.4. Sistema Solar actual.

asignó el nombre de Haumea ${ }^{4}$. Este objeto es un miembro del Disco Dispersado de la Región Transneptuniana, con un semieje mayor $a=43.2$ UA, una excentricidad $e=0.19$ y una inclinación $i=28.2^{\circ}$ (Brown et al. 2008). De acuerdo a los estudios desarrollados por Brown et al. (2005a), Haumea (2003 EL61) posee una densidad de $2.6 \mathrm{~g} \mathrm{~cm}^{-3}$ y un tamaño de 2000 $\times 1500 \times 1000 \mathrm{~km}$. "Haumea" es la diosa del nacimiento y la fertilidad en la mitología de Hawaii. Este nombre, elegido por D. L. Rabinowitz, es particularmente adecuado ya que la diosa Haumea también representa a la piedra y las observaciones de 2003 EL61 sugieren que este objeto está compuesto casi totalmente de roca con una corteza de hielo puro.

La Figura 2.4 nos muestra un esquema representativo de nuestro Sistema Solar actual, ilustrando los ocho Planetas y los cinco Planetas Enanos clasificados hasta la fecha.

\footnotetext{
${ }^{4}$ Debemos tener presente que existe una enorme controversia relacionada con el descubrimiento de Haumea. En efecto, un equipo de trabajo encabezado por Michael Brown, del Instituto de Tecnología de California, y un grupo español dirigido por José Luis Ortiz, del Instituto de Astrofísica de Andalucía, se disputan el crédito por el descubrimiento de este objeto.
} 



\section{Capítulo 3}

\section{Generalidades}

\subsection{Estructura Dinámica y Distribución Orbital}

\subsubsection{Resonancias en el Sistema Solar}

La estructura dinámica de nuestro Sistema Solar está determinada fundamentalmente por el fenómeno de resonancia. En términos generales, una resonancia puede ocurrir cuando existe una simple relación numérica entre frecuencias o períodos. Uno de los casos más conocidos de resonancias en el Sistema Solar está dado por el acoplamiento spin-órbita, en el cual están involucrados los períodos rotacional y orbital de un dado cuerpo. El ejemplo más obvio de acoplamiento spin-órbita está dado por la Luna, la cual tiene un período orbital que es igual a su período rotacional. Este hecho hace que nuestro satélite natural muestre siempre la misma cara a la Tierra.

Aquí, nosotros estamos interesados fundamentalmente en describir las principales características de las resonancias de movimientos medios y las resonancias seculares en nuestro Sistema Solar. Un análisis detallado de estas cuestiones puede ser encontrado en Murray \& Dermott (1999).

\section{Resonancias de Movimientos Medios}

Una resonancia de movimientos medios tiene lugar cuando existe una conmensurabilidad entre los períodos orbitales de dos o más cuerpos. Para analizar en detalle las principales características asociadas a este mecanismo resonante, consideremos la siguiente situación: supongamos que tenemos una masa $m$ la cual se mueve alrededor de una masa central $m_{\mathrm{c}}$ describiendo una órbita elíptica. Como es sabido, este problema de dos cuerpos resulta ser absolutamente integrable y los elementos orbitales semieje mayor $a$, excentricidad $e$, inclinación $i$, longitud del perihelio $\bar{\omega}$ y longitud del nodo ascendente $\Omega$ son cantidades constantes. Si se introduce una tercera masa $m^{\prime}$, el problema se vuelve no integrable. Sin embargo, es posible cuantificar la acción perturbadora de la masa $m^{\prime}$ sobre la masa $m$ a través del gradiente de una función potencial conocida como Función Perturbadora.

La Función Perturbadora de la masa $m$ generada por la masa $m^{\prime}$ adopta una expresión 
de la forma

$$
R=G m^{\prime} \sum_{j_{k}} S\left(a, a^{\prime}, e, e^{\prime}, i, i^{\prime}\right) \cos \phi
$$

donde $G$ es la constante de gravitación universal, $S$ es una función de los elementos orbitales $(a, e, i)$ y $\left(a^{\prime}, e^{\prime}, i^{\prime}\right)$ asociados a $m$ y $m^{\prime}$, respectivamente, y $\phi$ es un argumento cuya forma general está dada por

$$
\phi=j_{1} \lambda^{\prime}+j_{2} \lambda+j_{3} \bar{\omega}^{\prime}+j_{4} \bar{\omega}+j_{5} \Omega^{\prime}+j_{6} \Omega
$$

donde $(\lambda, \bar{\omega}, \Omega)$ y $\left(\lambda^{\prime}, \bar{\omega}^{\prime}, \Omega^{\prime}\right)$ representan la longitud media, la longitud del perihelio y la longitud del nodo ascendente asociadas a $m \mathrm{y}^{\prime}$, respectivamente. De acuerdo a la invariancia azimutal del potencial central, los enteros $j_{k}$ son tales que

$$
\sum_{k=1}^{6} j_{k}=0 .
$$

La longitud media $\lambda$ está dada por

$$
\begin{aligned}
\lambda & =M+\bar{\omega} \\
& =n(t-\tau)+\bar{\omega} \\
& =n t+\epsilon,
\end{aligned}
$$

donde $M$ es la anomalía media, $n$ es el movimiento medio, $\tau$ es el tiempo de pasaje por el perihelio y $\epsilon$ es la longitud media de la época. A partir de esto, la variación en el tiempo de la longitud media $\lambda$ viene dada por

$$
\dot{\lambda}=n+\dot{n} t+\dot{\epsilon} .
$$

Si definimos una nueva longitud media de la época $\epsilon^{*}$ a través de la expresión

$$
\dot{\epsilon^{*}}=\dot{n} t+\dot{\epsilon}
$$

entonces

$$
\dot{\lambda}=n+\dot{\epsilon}^{*}
$$

con lo cual, la longitud media $\lambda$ puede ser escrita como

$$
\lambda=\int n d t+\epsilon^{*}
$$

De acuerdo con esto y la Ecuación 3.2, la variación temporal del argumento $\phi$ está dada por la expresión

$$
\dot{\phi}=j_{1}\left(n^{\prime}+\epsilon^{i *}\right)+j_{2}\left(n+\epsilon^{*}\right)+j_{3} \dot{\bar{\omega}}+j_{4} \dot{\bar{\omega}}+j_{5} \dot{\Omega}^{\prime}+j_{6} \dot{\Omega}
$$

Diremos que un cuerpo perturbado está en una resonancia exacta cuando la variación en el tiempo de un argumento resonante particular, $\dot{\phi}$, es igual a cero. Esto implica que en este caso 
existe una combinación lineal de movimientos medios y tasas de precesión de las longitudes, tales que, para un conjunto adecuado de enteros $j_{k}$, se cumple que $\dot{\phi}=0$.

A partir de esta definición y la Ecuación 3.9, resulta evidente que si despreciamos las contribuciones de las tasas de precesión de las longitudes, la resonancia exacta ocurre cuando

$$
j_{1} n^{\prime}+j_{2} n \approx 0
$$

conduciendo a una conmensurabilidad de movimientos medios entre la masa perturbada $m$ y la masa perturbadora $m^{\prime}$. Si $m^{\prime}$ representa un cuerpo externo a $m$, y si llamamos $j_{1}=p+q$ y $j_{2}=-p$, con $p$ y $q$ enteros positivos, entonces $q$ nos define el orden de la resonancia. Se define la localización nominal, $a_{\mathrm{n}}$, de la resonancia $p+q: p$ al semieje mayor del objeto perturbado interno $m$ que satisface la relación

$$
a_{\mathrm{n}}=\left(\frac{p}{p+q}\right)^{2 / 3} a^{\prime} .
$$

Sin embargo, siempre es importante tener en cuenta que esta expresión sólo nos da una aproximación para la localización de la resonancia. En efecto, en aquellos casos donde las contribuciones por parte de las tasas de precesión de las longitudes no puedan ser despreciadas en la expresión de $\dot{\phi}$, las localizaciones de las diferentes resonancias exactas asociadas con la conmensurabilidad $p+q: p$ serán distintas.

La dinámica de un objeto en una resonancia está caracterizada por el comportamiento del argumento resonante $\phi$. Si dicho objeto se encuentra en la resonancia, $\phi$ experimenta libraciones periódicas alrededor de un valor fijo, mientras que si el objeto no está en resonancia, $\phi$ circula. Para interpretar un poco mejor el significado del argumento resonante $\phi$ consideremos el caso donde el objeto perturbado interno de masa $m$ y el objeto perturbador externo de masa $m^{\prime}$ se mueven en el mismo plano de referencia $\left(\Omega=\Omega^{\prime}=0\right)$, y además este ultimo lo hace sobre una órbita circular $\left(e^{\prime}=\bar{\omega}^{\prime}=0\right)$. En este escenario, el argumento resonante $\phi$ dado por la Ecuación 3.2 toma la forma

$$
\phi=j_{1} \lambda^{\prime}+j_{2} \lambda+j_{4} \bar{\omega}
$$

Nuevamente, si llamamos $j_{1}=p+q$ y $j_{2}=-p$, con $p$ y $q$ enteros positivos, la Ecuación 3.3 indica que $j_{4}=-q$, con lo cual se pone en evidencia que el valor absoluto del coeficiente $j_{4}$ coincide con el orden de la resonancia. Con esto, el argumento resonante $\phi$ se escribe como

$$
\phi=(p+q) \lambda^{\prime}-p \lambda-q \bar{\omega} .
$$

En una conjunción entre los objetos de masa $m \mathrm{y}^{\prime}, \lambda=\lambda^{\prime}=\lambda_{\text {conj }}$, con lo cual

$$
\phi=q\left(\lambda_{\text {conj }}-\bar{\omega}\right)
$$

Esta relación pone en evidencia que el argumento resonante $\phi$ es una medida de la longitud media a la cual se produce la conjunción entre los dos cuerpos involucrados, tomando como origen el perihelio de la órbita del objeto perturbador externo. De este modo, si un objeto está en una resonancia y su argumento resonante $\phi$ libra alrededor de los $0^{\circ}$, esto indica que las conjunciones con el cuerpo perturbador se producen alrededor del perihelio. En este escenario, el cuerpo perturbado de masa $m$ experimenta encuentros cercanos periódicos con 
el objeto perturbador de masa $m^{\prime}$, generando una configuración inestable. En efecto, como consecuencia de las perturbaciones a las cuales da lugar cada encuentro cercano, el objeto perturbado terminará escapando de la resonancia. Por el contrario, si un objeto está en una resonancia y su argumento resonante $\phi$ experimenta pequeñas libraciones alrededor de los $180^{\circ}$, esto indica que las conjunciones con el cuerpo perturbador se producen alrededor del afelio. En este escenario, la resonancia proporciona un mecanismo de protección, evitando que el cuerpo perturbado de masa $m$ sufra encuentros cercanos con el objeto perturbador de masa $m^{\prime}$. Sin embargo, si la amplitud de la libración del argumento $\phi$ alrededor de los $180^{\circ}$ resulta ser significativa, los encuentros cercanos llegan a ser más probables. De este modo, el mecanismo de protección de la resonancia se vuelve cada vez menos eficiente con un incremento en la amplitud de libración del argumento resonante.

El argumento $\phi$ de las partículas en la mayoría de las resonancias de movimientos medios libra alrededor de $180^{\circ}$. Sin embargo, para resonancias del tipo 1: $k$, el centro de libración del argumento resonante es dependiente de la excentricidad y se bifurca en múltiples centros de libración asimétricos (Beaugé 1994). En efecto, a excentricidades no muy pequeñas, existen dos centros de libración estables alrededor de $180^{\circ} \pm \delta$, con $\delta \sim 60^{\circ}$, mientras que las libraciones alrededor de $180^{\circ}$ resultan ser inestables.

Un punto de interés a tener en cuenta indica que el poder de una resonancia particular resulta ser proporcional a $e^{|q|}$, siendo $e$ la excentricidad de la partícula resonante y q el orden de la resonancia. Este resultado nos permite inferir que las resonancias de movimientos medios de más bajo orden resultan ser las más poderosas, mientras que aquellas de órdenes mayores producen un efecto menor.

\section{Resonancias Seculares}

Las resonancias seculares ocurren cuando la tasa de precesión de la longitud del perihelio o de la longitud del nodo de un objeto resultan equivalentes a la tasa de precesión media de la longitud del perihelio o de la longitud del nodo de uno de los planetas. Las resonancias seculares que involucran las tasas de precesión de la longitud del perihelio excitan las excentricidades, mientras que aquellas que involucran las tasas de precesión de la longitud del nodo excitan las inclinaciones (Williams \& Faulkner 1981; Morbidelli \& Henrard 1991).

Para objetos sobre órbitas altamente inclinadas existe otra forma de resonancia secular, aunque en este caso no se involucra a ninguna frecuencia del sistema planetario. Se trata de la resonancia de Kozai, la cual ocurre cuando $\dot{\omega}=0$, siendo $\omega$ el argumento del perihelio. Kozai (1962) mostró que un asteroide perturbado por Júpiter moviéndose sobre una órbita circular no tendría cambios seculares en su semieje mayor pero su excentricidad e inclinación podrían sufrir cambios tales que la cantidad

$$
H_{\mathrm{K}}=\sqrt{1-e^{2}} \cos i,
$$

siempre permanece constante. Una consecuencia inmediata de la conservación de esta cantidad es que la excentricidad e inclinación de la órbita del objeto están acopladas de modo tal que $e$ alcanza un máximo cuando $i$ toma un valor mínimo y vice versa. Michel \& Thomas (1995) extendieron la teoría de Kozai con el fin de tener en cuenta el efecto de los cuatro planetas gigantes. El estudio indica que para bajas inclinaciones $\omega$ libra alrededor de los puntos de equilibrio estable localizados en $\omega=0^{\circ}$ y $\omega=180^{\circ}$. Para inclinaciones mayores a $\sim 30^{\circ}$, estos 
puntos llegan a ser inestables y los nuevos puntos de equilibrio estable aparecen localizados en $\omega=90^{\circ}$ y $\omega=270^{\circ}$. Thomas \& Morbidelli (1996) mostraron que la resonancia de Kozai sólo puede afectar órbitas con grandes excentricidades e inclinaciones.

\subsubsection{Conexión Dinámica entre los Asteroides del Cinturón Principal y los NEOs}

El Cinturón Principal de asteroides es una vasta región localizada entre Marte y Júpiter, aproximadamente desde las 2 hasta las 3.4 UA del Sol. Los valores medios de la excentricidad e inclinación de los objetos de este cinturón son de $\langle e\rangle \sim 0.14$ y $\langle i\rangle \sim 12^{\circ}$, respectivamente, los cuales resultan ser mayores que aquellos correspondientes a los planetas, con la excepción de Mercurio. Valores tan elevados para estos parámetros orbitales no permiten sostener un proceso de acreción efectivo, lo cual justifica la ausencia de un único objeto de mayor tamaño en esta región del Sistema Solar.

Los objetos cercanos a la Tierra (Near-Earth Objects "NEOs") representan otra importante población, la cual incluye asteroides (Near-Earth Asteroids "NEAs"), cometas activos y cometas extintos (Near-Earth Comets "NECs"). Los NEOs tienen distancias perihélicas $q \leq 1.3$ UA y distancias afélicas $Q \geq 0.983$ UA (Rabinowitz et al. 1994). Estos objetos son divididos generalmente en tres subcategorías que incluyen los Atenas $(a<1 \mathrm{UA}, Q \geq 0.983$ UA) y los Apolos ( $a \geq 1 \mathrm{UA}, q \leq 1,0167 \mathrm{UA}$ ), los cuales están sobre órbitas que cruzan la correspondiente a la Tierra, y los Amor (1.0167 UA $<q \leq 1,3$, los cuales se mueven sobre órbitas cercanas a la de nuestro planeta. De acuerdo a lo sugerido por Bottke et al. (2002), aproximadamente el $6 \%$ de los NEOs son NECs. Dado que la contribución de los cometas a la población de NEOs resulta ser poco significativa, la misma no será tenida en cuenta en esta Tesis. Como consecuencia, en el análisis desarrollado en el Capítulo 5, asumiremos que los NEAs constituyen la totalidad de la población de objetos cercanos a la Tierra.

La Figura 3.1 muestra la distribución de asteroides del Cinturón Principal y NEOs respecto del semieje mayor $a$, excentricidad $e$ e inclinación $i$.

Los asteroides del Cinturón Principal y los NEOs están íntimamente conectados por procesos evolutivos y mecanismos de transporte dinámicos asociados a las resonancias orbitales. Desde el trabajo de Williams (1969) y demás estudios desarrollados por Wetherill (1979) y Wisdom $(1983,1985 \mathrm{a}, 1985 \mathrm{~b})$, es ampliamente aceptado que las regiones resonantes presentes en el Cinturón Principal actúan como rutas de escape efectivas. En efecto, las simulaciones numéricas desarrolladas por Gladman et al. (1997) han mostrado que aquellos objetos que caen en alguna resonancia dentro de las 2.5 UA podrían llegar a ser NEOs y luego meteoritos en sólo unos pocos millones de años, siendo el estado final más común para estos objetos un impacto con el Sol. Ellos también han mostrado que aquellos cuerpos que alcanzan una de las regiones resonantes más allá de las 2.5 UA llegan a ser cruzadores de Júpiter y son subsecuentemente removidos del Sistema Solar por encuentros cercanos con dicho planeta. La intensa actividad colisional presente en el Cinturón Principal quiebra continuamente a los asteroides, inyectando una gran cantidad de material dentro de las resonancias, dando lugar a un mecanismo que representa una fuente de pérdida de masa en dicha región.

Otro de los mecanismos que juega un rol relevante en la evolución de estos pequeños cuerpos es el denominado efecto Yarkovsky. El efecto Yarkovsky es el resultado de una fuerza de radiación que modifica los parámetros orbitales de los asteroides, dando lugar a un mecanismo 

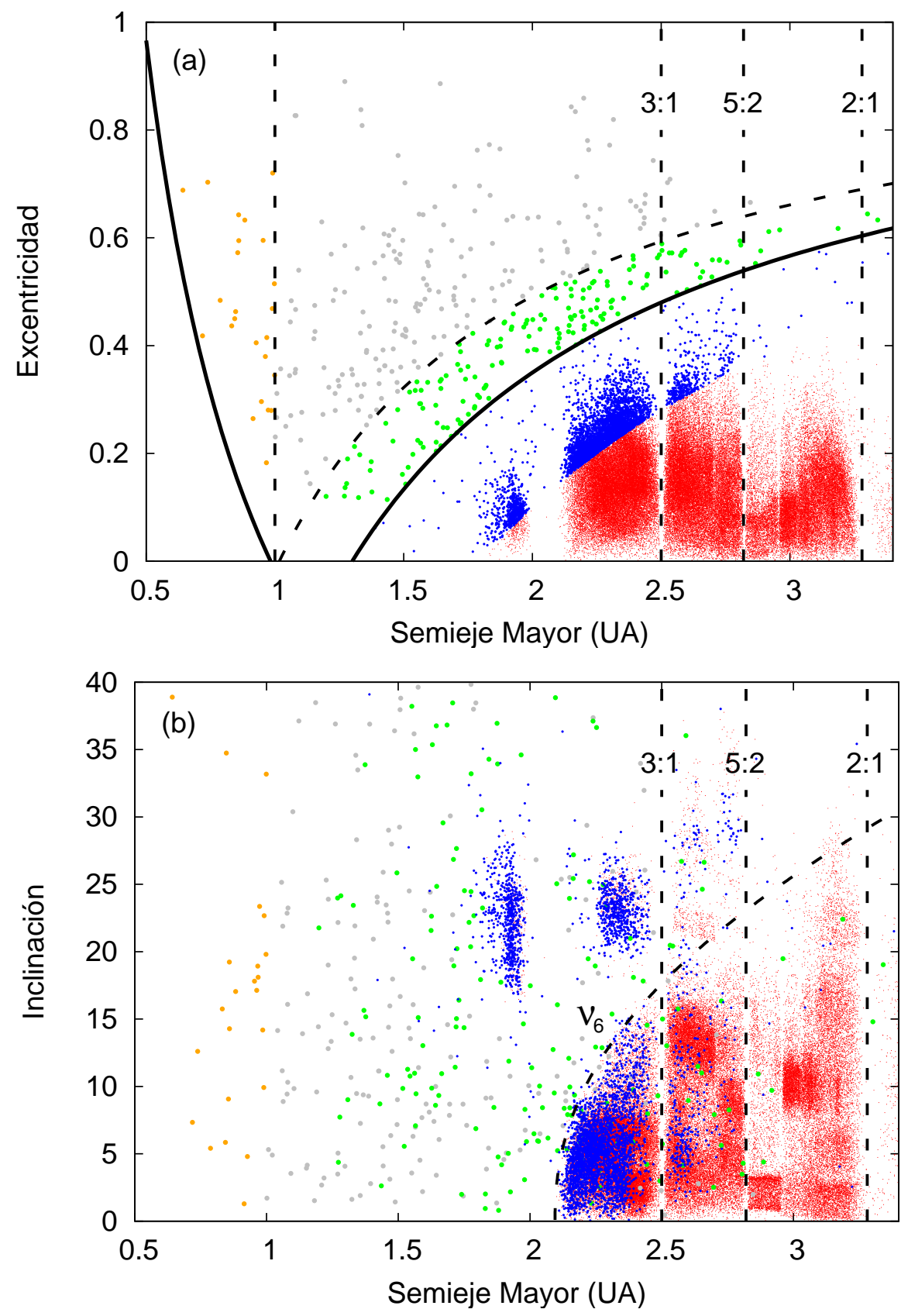

Figura 3.1. Distribución de los asteroides del Cinturón Principal, Cruzadores de Marte y NEOs con respecto al semieje mayor, excentricidad e inclinación. Los asteroides del Cinturón Principal y los Cruzadores de Marte son representados por puntos de color rojo y azul, respectivamente. Las curvas sólidas delimitan la región ocupada por los NEOs. Los Atenas, Apolos y Amor son representados por puntos de color naranja, gris y verde, respectivamente. Mientras que la línea de trazos vertical determina el límite entre las regiones ocupadas por los Atenas y Apolos, la curva de trazos representa la separación entre los Apolos y Amor. Las resonancias de movimientos medios con Júpiter 3:1, 5:2 y 2:1 son mostradas en ambas figuras. Por otra parte, la resonancia secular $\nu_{6}$ sólo es representada sobre el panel inferior ya que su posición depende de la inclinación orbital y sólo muy débilmente de la excentricidad. (Datos extraídos de http://ssd.jpl.nasa.gov/dat/ELEMENTS.NUMBR). 
que puede llevarlos dentro de las resonancias y de este modo removerlos del Cinturón Principal. Además, este efecto es dependiente del tamaño y debido a esto, su acción acoplada con la presencia de las regiones resonantes no sólo puede ser una importante fuente de pérdida de masa en el Cinturón Principal, sino que además también puede afectar su distribución de tamaños. Existen fuertes evidencias asociadas a la distribución de tamaños de los NEOs que sugieren que el efecto Yarkovsky juega un rol dominante en el transporte de material dentro de las resonancias del Cinturón Principal, siendo más relevante que la inyección colisional (Morbidelli et al. 2002; Morbidelli \& Vokrouhlický 2003).

Las resonancias orbitales entre los asteroides y los planetas así como también el efecto Yarkovsky adquieren una importancia fundamental ya que los mismos llevan a una conexión entre los asteroides del Cinturón Principal y la población de NEOs.

Morbidelli et al. (2002) analizaron las principales características dinámicas de las resonancias orbitales en el Cinturón Principal y sugirieron distinguir entre resonancias poderosas $\mathrm{y}$ resonancias difusivas.

Las resonancias poderosas están caracterizadas por la existencia de gaps bien definidos en la distribución de asteroides del Cinturón Principal. La resonancia secular $\nu_{6}$, la cual determina el límite interior del cinturón de asteroides, y las resonancias de movimientos medios con Júpiter 3:1, 5:2 y 2:1 a 2.5, 2.8 y 3.27 UA del Sol, respectivamente, representan las más importantes resonancias de esta clase (Figura 3.1). A continuación, brindamos las más importantes propiedades dinámicas correspondientes a cada una de estas regiones resonantes (Morbidelli et al. 2002):

- Resonancia $\nu_{6}$ : La resonancia secular $\nu_{6}$ ocurre cuando la tasa de precesión de la longitud del perihelio del asteroide coincide con la tasa media de precesión de la longitud del perihelio de Saturno. El efecto de esta resonancia decrece rápidamente con la distancia a la curva de trazos mostrada en la Figura 3.1b. En efecto, al costado derecho de la misma es posible distinguir una "región fuerte" y una "región débil", cada una ocupando una extensión de 0.04 UA.

En la región fuerte, la resonancia genera un gran incremento de la excentricidad de los asteroides. Como consecuencia de esto, estos objetos alcanzan órbitas que cruzan la Tierra o Venus, y en diversos casos ellos colisionan con el Sol, adquiriendo distancias perihélicas menores al radio solar. El tiempo medio requerido por estos objetos para llegar a ser "cruzadores de la Tierra" es de $\sim 0.5 \times 10^{6}$ años. De acuerdo a lo analizado por Gladman et al. (1997), el tiempo de vida medio de los cuerpos residentes en la resonancia $\nu_{6}$ es de $2 \times 10^{6}$ años, mientras que los estados finales típicos de dichos objetos son un impacto con el Sol (en el $80 \%$ de los casos) y una eyección sobre una órbita hiperbólica (en sólo el $12 \%$ de los casos). Según lo sugerido por Bottke et al. (2002), el tiempo medio de residencia en la región de los NEOs por un objeto proveniente de la resonancia $\nu_{6}$ es de $6.5 \times 10^{6}$ años. Por otra parte, de acuerdo a lo indicado por Morbidelli \& Gladman (1998), la probabilidad media de colisión con la Tierra de un cuerpo inicialmente en la resonancia $\nu_{6}$, integrada sobre su tiempo de vida en la región de cruce con nuestro planeta, es de $\sim 10^{-2}$.

En la región débil, el efecto de la resonancia $\nu_{6}$ decrece pero aun es capaz de forzar a los asteroides a cruzar la órbita de Marte. Para ingresar a la región de los NEOs, estos objetos deben evolucionar bajo el efecto de sucesivos encuentros con Marte y, de 
acuerdo a lo estudiado por Morbidelli \& Gladman (1998), el tiempo requerido para ello se incrementa fuertemente con la distancia desde la resonancia. El comportamiento dinámico de los asteroides en esta región resulta ser muy complejo debido a que la misma se encuentra densamente poblada por diversas resonancias de movimientos medios con Marte.

- Resonancia 3:1: La resonancia de movimientos medios 3:1 con Júpiter está localizada a $\sim 2.5$ UA del Sol. Dentro de esta resonancia es posible distinguir dos regiones dinámicamente diferentes: 1) una región central angosta donde los asteroides sufren oscilaciones regulares de sus excentricidades, lo que los lleva a cruzar periódicamente la órbita de Marte, y 2) una región exterior más grande donde la evolución de la excentricidades es ampliamente caótica, por lo que los cuerpos pueden rápidamente cruzar la órbita de la Tierra e incluso impactar con el Sol. Como consecuencia de los sucesivos encuentros con Marte, los cuerpos residentes en la región central pueden fácilmente ser transferidos a la región exterior y luego rápidamente depositados en la región de los NEOs. El tiempo medio requerido por estos objetos para llegar a ser "cruzadores de la Tierra" es de $\sim 10^{6}$ años. De acuerdo a lo estudiado por Gladman et al. (1997), el tiempo de vida medio de los cuerpos residentes en la resonancia 3:1 es de $\sim 2 \times 10^{6}$ años, mientras que los estados finales típicos de dichos objetos son un impacto con el Sol (en el $70 \%$ de los casos) y una eyección sobre una órbita hiperbólica (en el $28 \%$ de los casos). Según lo indicado por Bottke et al. (2002), el tiempo medio de residencia en la región de los NEOs por un objeto proveniente de la resonancia $3: 1$ es de $2.2 \times 10^{6}$ años. Por otra parte, de acuerdo a lo indicado por Morbidelli \& Gladman (1998), la probabilidad media de colisión con la Tierra de un cuerpo inicialmente en la resonancia 3:1, integrada sobre su tiempo de vida en la región de cruce con nuestro planeta, es de $\sim 2 \times 10^{-3}$.

- Resonancia 5:2: La resonancia de movimientos medios 5:2 con Júpiter está localizada $\mathrm{a} \sim 2.8$ UA del Sol. Según los analizado por Moons \& Morbidelli (1995), esta resonancia resulta ser globalmente caótica. Como consecuencia de esto, la resonancia 5:2 es aquella que incrementa las excentricidades orbitales de los asteroides sobre la escala de tiempo más corta. El tiempo medio requerido por estos objetos para llegar a ser "cruzadores de la Tierra" es de $\sim 0.3 \times 10^{6}$ años. De acuerdo a lo indicado por Gladman et al. (1997), el tiempo de vida medio de los cuerpos residentes en la resonancia 5:2 es de $5 \times 10^{5}$ años, mientras que el estado final más representativo de dichos objetos es una eyección sobre una órbita hiperbólica (en el $92 \%$ de los casos), ya que dicha resonancia se encuentra mucho más cerca de Júpiter que las otras resonancias poderosas previamente analizadas. Según lo indicado por Bottke et al. (2002), el tiempo medio de residencia en la región de los NEOs por un objeto proveniente de la resonancia 5:2 es de $4 \times 10^{5}$ años. Por otra parte, de acuerdo a lo indicado por Morbidelli \& Gladman (1998), la probabilidad media de colisión con la Tierra de un cuerpo inicialmente en la resonancia $5: 2$, integrada sobre su tiempo de vida en la región de cruce con nuestro planeta, es de $\sim 2.5 \times 10^{-4}$.

- Resonancia 2:1: La resonancia de movimientos medios 2:1 con Júpiter está localizada $\mathrm{a} \sim 3.27$ UA del Sol. Los estudios desarrollados por Nesvorný \& Ferraz-Melo (1997) y Moons et al.(1998) establecen que la estructura dinámica de la resonancia 2:1 es muy complicada. En el centro de la resonancia y a excentricidades moderadas, existe una región estable. Por otra parte, las regiones cercanas a los bordes de la resonancia resultan 
ser inestables aunque algunos millones de años son requeridos para que los objetos allí residentes lleguen a ser "cruzadores de la Tierra". Las simulaciones numéricas desarrolladas por Gladman et al. (1997) sugieren que el tiempo de vida medio de los cuerpos inicialmente en la resonancia 2:1 es mayor a $10^{8}$ años. Sin embargo, el trabajo de Brož et al. (2005), el cual reexamina el origen, la evolución y la supervivencia de los objetos en la resonancia 2:1, indica que el efecto Yarkovsky continuamente suministra objetos a esta región resonante y conserva la población inestable en un estado aproximadamente estacionario, obteniendo tiempos de vida que van desde $\sim 10^{6}$ a $10^{8}$ años con un valor medio de $\sim 10^{7}$ años. De este modo, la resonancia 2:1 es capaz de perturbar el movimiento de los asteroides sobre escalas de tiempo comparables a las de las otras resonancias poderosas anteriormente analizadas. Según lo indicado por Bottke et al. (2002), el tiempo medio de residencia en la región de los NEOs por un objeto proveniente de la resonancia 2:1 es de $\times 10^{5}$ años, ya que los mismos son rápidamente eyectados por Júpiter sobre órbitas hiperbólicas. Por otra parte, de acuerdo a lo indicado por Morbidelli et al. (2002), la probabilidad media de colisión con la Tierra de un cuerpo inicialmente en la resonancia 2:1, integrada sobre su tiempo de vida en la región de cruce con nuestro planeta, es de $\sim 5 \times 10^{-5}$.

A diferencia las resonancias poderosas descriptas anteriormente, las resonancias difusivas no tienen profundos gaps asociados en la distribución de los asteroides del Cinturón Principal. Existen cientos de estas resonancias débiles que cruzan densamente el Cinturón Principal. Las mismas están representadas por:

- resonancias de movimientos medios con Júpiter de alto orden,

- resonancias de tres cuerpos con Júpiter y Saturno (Murray et al. 1998; Nesvorný \& Morbidelli 1998, 1999), y

- resonancias de movimientos medios con Marte (Morbidelli \& Nesvorný 1999).

La existencia de las resonancias difusivas lleva a que muchos de los asteroides del Cinturón Principal presenten un comportamiento caótico (Nesvorný et al. 2002a), aunque el efecto de esta caoticidad resulta ser muy débil. Estas resonancias pueden producir lentos cambios en los parámetros orbitales de los objetos, llevándolos a evolucionar hacia órbitas que cruzan los planetas. Particularmente en la región interna del cinturón de asteroides, las resonancias difusivas pueden explicar la existencia de una población distintiva de pequeños cuerpos conocida como Cruzadores de Marte (Figura 3.1). De acuerdo a lo establecido por Migliorini et al. (1998), los Cruzadores de Marte están definidos como aquellos cuerpos con una distancia perihélica $q>1,3$ UA y una combinación de parámetros orbitales $(a, e, i)$ tales que ellos cruzan la órbita de Marte durante un ciclo de oscilación secular de su excentricidad.

La Figura 3.1 muestra que la población de Cruzadores de Marte se extiende fundamentalmente hasta las 2.8 UA, sugiriendo que el fenómeno de difusión caótica en el Cinturón Principal se extiende al menos hasta este límite. Por otra parte, la Figura 3.1b muestra que además de la población principal de Cruzadores de Marte $^{1}$, situada por debajo de la resonancia secular $\nu_{6}$, existen otros dos grupos pequeños de Cruzadores de Marte con alta inclinación.

\footnotetext{
${ }^{1}$ Bottke et al. (2002) llamaron a esta población Intermediate Source Mars-Crossers y por ende, de aquí en adelante la misma será referida bajo la sigla IMC
} 


\begin{tabular}{|c|c|c|c|c|c|c|}
\hline & $\nu_{6}$ & IMC & $3: 1$ & OB & JFC & Total \\
\hline $\mathrm{N}_{\mathrm{NEA}}(\mathrm{H}<18)$ & $360 \pm 90$ & $240 \pm 40$ & $220 \pm 90$ & $79 \pm 12$ & $61 \pm 43$ & $960 \pm 120$ \\
\hline$\tau\left(\right.$ millón de años $\left.{ }^{-1}\right)$ & $55 \pm 18$ & $65 \pm 15$ & $100 \pm 50$ & $570 \pm 120$ & - & $790 \pm 200$ \\
\hline $\mathrm{T}_{\mathrm{NEA}}$ (millón de años) & 6.54 & 3.75 & 2.16 & 0.14 & - & - \\
\hline
\end{tabular}

Tabla 3.1. Algunos de los más importantes resultados dinámicos derivados por Bottke et al. (2002) para cuerpos provenientes desde las diferentes regiones fuentes de NEOs. Las fuentes estudiadas por estos autores fueron las resonancias $\nu_{6}$ y 3:1, la población principal de Cruzadores de Marte (IMC), el Cinturón Principal exterior (OB) y la población de Cometas de la Familia de Júpiter $(\mathrm{JFC}) . \mathrm{N}_{\mathrm{NEA}}(\mathrm{H}<18)$ representa el número de NEOs en estado estacionario con una magnitud $\mathrm{H}$ menor a 18 , lo cual equivale a $\sim 1 \mathrm{~km}$ de diámetro. Por otra parte, $\tau$ (millón de años ${ }^{-1}$ ) da el número de cuerpos inyectados dentro de la región de los NEOs por millón de años, mientras que $\mathrm{T}_{\mathrm{NEA}}$ (millón de años) representa el tiempo de vida dinámico medio de los objetos en dicha región.

Ellos son los Hungaria, con $1.77<a<2.06$ UA e $i>15^{\circ}$, y los Phocaea con $2.1<a<2.5$ UA e $i>18^{\circ}$. La existencia de estos dos pequeños grupos confirma que la difusión caótica también resulta ser efectiva en estas regiones de elevada inclinación.

Los Cruzadores de Marte sufren sucesivos encuentros con este planeta, experimentando variaciones en sus semiejes mayores. Este fenómeno tiene lugar hasta que estos objetos son capturados dentro de alguna resonancia lo suficientemente fuerte como para disminuir sus distancias perihélicas por debajo de las 1.3 UA, y luego alcanzar una órbita de cruce con la de la Tierra. Michel et al. (2000) desarrollaron simulaciones numéricas de la evolución dinámica de objetos sobre órbitas que cruzan Marte. Estos trabajos muestran que los Cruzadores de Marte de la población principal (IMC) pueden llegar a ser NEOs sobre una escala de tiempo de $\sim 6 \times 10^{7}$ años, mientras que los Cruzadores de Marte asociados a los pequeños grupos de alta inclinación requieren para ello tiempos mayores $\mathrm{a} \times 10^{8}$ años.

El escaso número de Cruzadores de Marte más allá de las 2.8 UA no es debido a la ineficiencia del proceso de difusión caótica en las regiones exteriores del Cinturón Principal. Por el contrario, esto es una consecuencia de que los tiempos de vida dinámicos de los Cruzadores de Marte decrece con un aumento del semieje mayor, debido a la acción perturbadora de Júpiter.

Bottke et al. (2002) estudiaron la distribución de magnitudes absolutas $\mathrm{H}^{2}$ y parámetros orbitales de la población de objetos cercanos a la Tierra. Para hacer esto, los autores desarrollaron una integración numérica de miles de partículas de prueba inicialmente colocadas en cinco diferentes regiones fuente de NEOs: la resonancia secular $\nu_{6}$, la resonancia de movimientos medios 3:1 con Júpiter, la población principal de Cruzadores de Marte (IMC), el Cinturón Principal Exterior con $a \geq 2.8$ UA y la población de Cometas de la Familia de Júpiter. La Tabla 3.1 resume algunos de los resultados dinámicos más importantes derivados por Bottke et al. (2002). A partir de los mismos es posible argumentar que la resonancia secular $\nu_{6}$, la población principal de Cruzadores de Marte (IMC) y la resonancia de movimientos medios 3:1 con Júpiter resultan ser fuentes primarias, generando de modo conjunto el $85 \%$ de la población de NEOs en estado estacionario. Por el contrario, el Cinturón Principal Exterior y

\footnotetext{
${ }^{2} H$ es definida como la magnitud visual que un objeto tendría si estuviese a 1 UA del Sol y de la Tierra, con un ángulo de fase igual a cero. El ángulo de fase está definido como el ángulo entre el Sol y la Tierra visto desde el objeto en cuestión.
} 

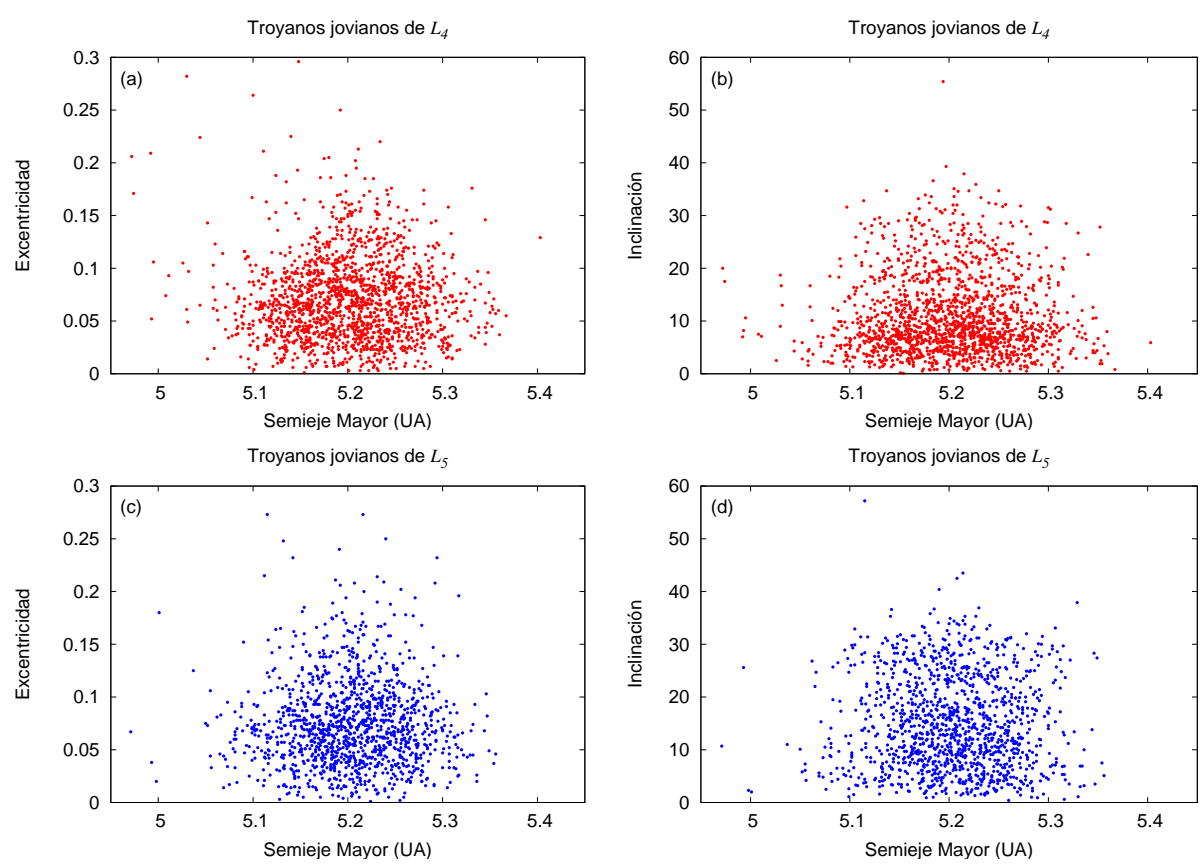

Figura 3.2. Distribución de los Troyanos jovianos de $L_{4}$ y $L_{5}$ respecto del semieje mayor, excentricidad e inclinación. No existen diferencias evidentes en las distribuciones orbitales de los dos enjambres.

la población de Cometas de la Familia de Júpiter deben ser consideradas sólo como fuentes secundarias.

\subsubsection{Troyanos Jovianos: Propiedades Orbitales y Características Dinámi- cas}

Los Troyanos jovianos son objetos localizados en la resonancia de movimientos medios 1:1 con Júpiter, librando alrededor de los puntos de equilibrio lagrangianos $L_{4}$ y $L_{5}$, a $60^{\circ}$ de longitud por delante y por detrás del planeta.

La Figura 3.2 expone la distribución de los Troyanos jovianos de $L_{4}$ y $L_{5}$ respecto del semieje mayor $a$, excentricidad $e$ e inclinación $i$. De las mismas se observa que las excentricidades de estos objetos se encuentran en el rango $0 \lesssim e \lesssim 0.3$, mientras que las inclinaciones se distribuyen entre los valores $0 \lesssim i \lesssim 60^{\circ}$. Por otra parte, la Figura 3.2 nos indica que no existen diferencias evidentes entre los parámetros orbitales asociados a cada uno de los dos enjambres.

Las órbitas de los Troyanos jovianos están caracterizadas por libraciones del ángulo $\lambda-\lambda_{\mathrm{J}}$, donde $\lambda$ y $\lambda_{\mathrm{J}}$ representan las longitudes medias del asteroide y Júpiter, respectivamente. De acuerdo a lo sugerido por Marzari et al. (2002, 2003), la amplitud de libración de los Troyanos jovianos de $L_{4}$ se ubica en un amplio rango de valores que van desde $0.6^{\circ}$ hasta $88.7^{\circ}$, con un valor medio alrededor de $32.7^{\circ}$. Por otra parte, los estudios indican que no existen diferencias significativas entre las distribuciones de amplitudes de libración asociadas a los puntos $L_{4}$ y $L_{5}$. La Figura 3.3 expone la distribución de amplitudes de libración para los Troyanos jovianos observados de $L_{4}$, obtenida por Marzari et al. (2003). 


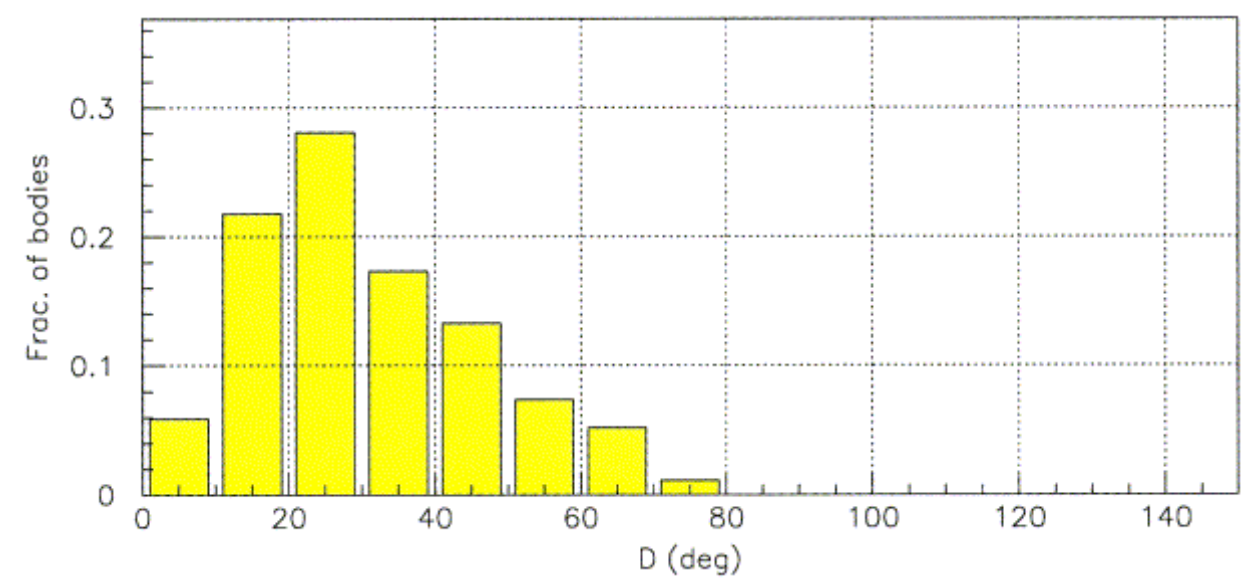

Figura 3.3. Distribución de amplitudes de libración para los Troyanos jovianos de $L_{4}$. Figura extraída de Marzari et al. (2003).

Un trabajo interesante concerniente a la evolución dinámica de la población de Troyanos jovianos fue desarrollado por Levison et al. (1997). Estos autores integraron numéricamente un conjunto de 270 partículas de prueba sin masa, bajo la influencia gravitacional del Sol y de los cuatro planetas gigantes, sobre una escala de tiempo de $10^{9}$ años. El resultado más importante derivado por estos autores establece que las nubes de Troyanos jovianos se están dispersando lentamente, sugiriendo que esta población debe ser incluida en la lista de estructuras dinámicamente inestables de nuestro Sistema Solar. Levison et al. (1997) encontraron una tasa de erosión dinámica desde los enjambres de Troyanos jovianos de $\sim 6.2 \times 10^{-5}$ objetos con diámetro $D \geq 1 \mathrm{~km}$ cada año.

\subsubsection{Estructura Dinámica de la Región Transneptuniana}

\section{Clases Dinámicas}

Los objetos de la Región Transneptuniana pueden ser clasificados en cuatro clases dinámicas bien diferenciadas:

- Objetos Resonantes: son aquellos que presentan alguna conmensurabilidad de movimientos medios con Neptuno. Si bien se han identificado objetos en diversas resonancias de orden $\leq 4$ (Elliot et al. 2005), la más densamente poblada de esta región resulta ser la resonancia de movimientos medios 3:2 con Neptuno, localizada a $\sim 39.5$ UA. Los objetos que residen en esta región resonante son llamados Plutinos, debido a la analogía de sus órbitas con aquella de Plutón, el cual es su miembro más representativo. Otra de las poblaciones resonantes distintivas de esta región está dada por los Troyanos de Neptuno, los cuales se encuentran localizados en una resonancia de movimientos medios 1:1 con dicho planeta. Estos objetos dejaron de ser una conjetura teórica a partir del año 2001, con el descubrimiento del primer Troyano de Neptuno, denominado 2001 QR322. Hasta la fecha se han observado un total de 6 objetos en esta población resonante, todos ellos asociados al punto de equilibrio $L_{4}$. 

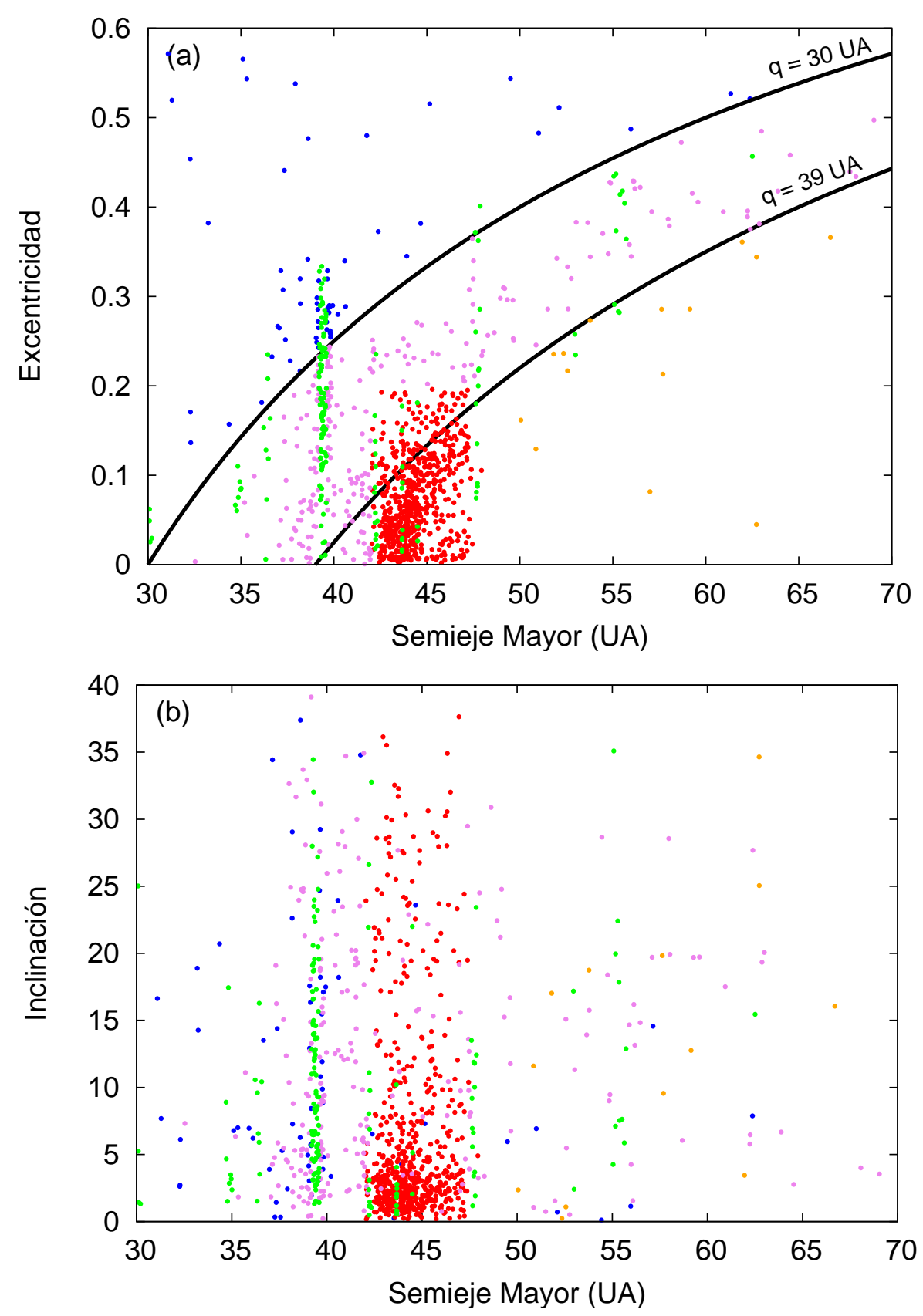

Figura 3.4. Distribución de Centauros y objetos de la Región Transneptuniana con respecto al semieje mayor, excentricidad e inclinación. Los Centauros, ubicados por encima de la curva sólida de distancia perihélica constante $q=30$ UA en a), están representados por puntos de color azul. Los Objetos Resonantes y Clásicos son ilustrados a través de puntos de color verde y rojo, respectivamente. Por otra parte, los objetos del Disco Dispersado y del Disco Dispersado Extendido son mostrados por puntos de color violeta y naranja, respectivamente. (Datos extraídos de http://ssd.jpl.nasa.gov/dat/ELEMENTS.NUMBR). 
- Objetos Clásicos: son aquellos que poseen semiejes mayores en el rango $42 \lesssim a \lesssim 48$ UA, excentricidades $e \lesssim 0.2$ y no presentan ninguna conmensurabilidad de movimientos medios con Neptuno. Los primeros objetos detectados pertenecientes a esta clase dinámica mostraron bajas inclinaciones orbitales. Sin embargo, los descubrimientos sucesivos permitieron inferir que existe un gran número de Objetos Clásicos en órbitas de elevada inclinación. Brown (2001) argumentó que la distribución real de inclinaciones $i$ de los Objetos Clásicos resulta ser bimodal y puede ser ajustada por la superposición de dos funciones gausianas, una con $\sigma \sim 2^{\circ}$ para $i \lesssim 4^{\circ}$ y otra con $\sigma \sim 12^{\circ}$ para $i \gtrsim 4^{\circ}$. A partir de este resultado, Brown (2001) sugirió que, de acuerdo al valor de sus inclinaciones, los Objetos Clásicos pueden ser clasificados en dos grupos diferenciados: la Población Cold, constituida por objetos con $i \lesssim 4^{\circ}$, y la Población Hot, representa por objetos con inclinaciones mayores.

- Objetos del Disco Dispersado: son aquellos que poseen distancias perihélicas en el rango $30 \lesssim q \lesssim 39$ UA, excentricidades $e \gtrsim 0.2$ y no presentan ninguna conmensurabilidad de movimientos medios con Neptuno. De acuerdo a Morbidelli et al. (2004), estos objetos serían los sobrevivientes de una población mucho más grande que fue rápidamente removida por las perturbaciones de Neptuno sobre la edad del Sistema Solar.

- Objetos del Disco Dispersado Extendido: son aquellos que poseen distancias perihélicas $q \gtrsim 39 \mathrm{UA}$, semiejes mayores $a \gtrsim 50$ UA y no presentan ninguna conmensurabilidad de movimientos medios con Neptuno. Las grandes distancias perihélicas características de estos objetos no pueden ser explicadas a partir de las perturbaciones gravitacionales ejercidas por Neptuno, al menos dentro del mismo escenario asociado a los objetos del Disco Dispersado (Gladman et al. 2002).

La Figura 3.4 expone la distribución de las diferentes clases dinámicas de la Región Transneptuniana respecto del semieje mayor, excentricidad e inclinación.

\section{Características Dinámicas Generales de la Región Transneptuniana}

Para analizar la estructura dinámica de la Región Transneptuniana, tomamos como referencia el trabajo de Morbidelli et al. (2008). Para realizar este estudio, presentamos la Figura 3.5, la cual muestra un mapa de los tiempos de vida dinámicos de los objetos residentes en dicha región del Sistema Solar exterior, como función de su semieje y excentricidad iniciales, para una inclinación de $i=1^{\circ}$ y con sus elipses orbitales orientadas al azar. Este mapa fue computado numéricamente por Duncan et al. (1995), simulando la evolución de un conjunto de partículas de prueba sin masa, bajo la acción de los cuatro planetas gigantes, los cuales fueron posicionados sobre sus órbitas actuales a lo largo de toda la integración. La simulación numérica fue extendida por $4 \times 10^{9}$ años y la evolución de cada partícula fue seguida hasta sufrir un encuentro cercano con Neptuno.

Los colores mostrados en la Figura 3.5 representan los diferentes tiempos requeridos para que una partícula encuentre a Neptuno, como una función de su semieje y excentricidad iniciales. Mientras que los colores obscuros indican zonas dinámicamente inestables de la Región Transneptuniana, el amarillo representa regiones estables donde los objetos pueden sobrevivir los $4 \times 10^{9}$ años de la simulación numérica, sin sufrir un encuentro cercano con Neptuno. Por otra parte, la Figura 3.5 muestra también la distribución de los objetos transneptunianos 


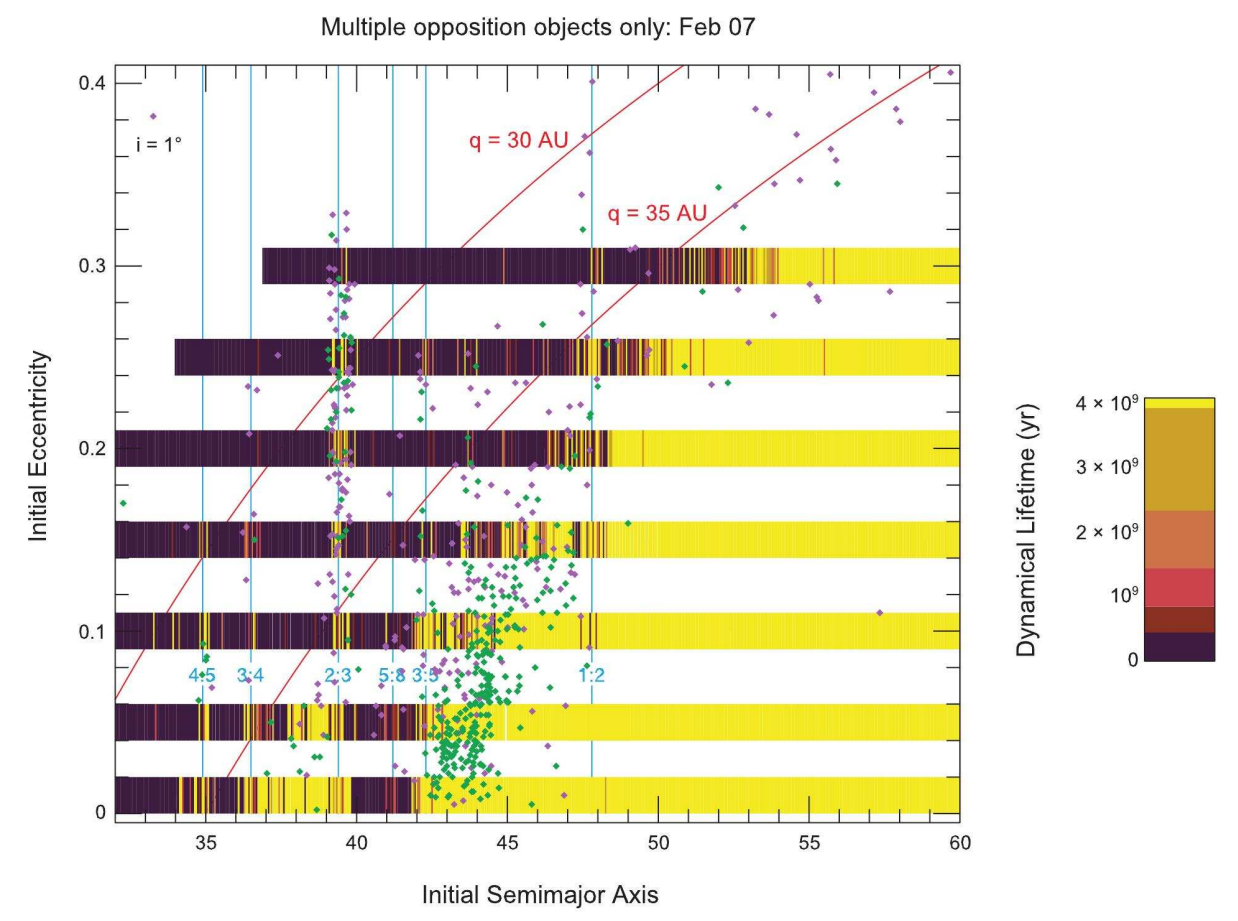

Figura 3.5. Tiempos de vida dinámicos obtenidos a partir de la integración numérica de un conjunto de partículas sin masa, extendida sobre $4 \times 10^{9}$ años. Cada color representa un tiempo de vida dinámico, de acuerdo a la tabla colocada a la derecha de la ilustración. Como referencia, se muestra la localización de las más importantes resonancias de movimientos medios con Neptuno, así como también dos curvas de distancia perihélica constante. Además, se grafica la distribución de objetos de la Región Transneptuniana observados sobre 3 oposiciones hasta el año 2007. Los puntos de color verde representan cuerpos con inclinaciones $i<4^{\circ}$, mientras que aquellos con inclinaciones mayores son ilustrados a través de puntos de color violeta. Figura realizada por Duncan et al.(1995), adaptada por Morbidelli et al. (2008). 
conocidos hasta el año 2007. Los puntos de color verde indican objetos con inclinaciones $i<$ $4^{\circ}$, mientras que aquellos de color violeta representan cuerpos con inclinaciones mayores.

Una rápida inspección de la Figura 3.5 nos muestra que la mayoría de los objetos con distancias perihélicas $q \lesssim 35$ UA y semiejes mayores $a<40$ UA resultan ser inestables, al igual que aquellos con distancias perihélicas $q \lesssim 37-38$ UA y semiejes mayores $42<a<$ 50 UA. Dicha inestabilidad es debida a Neptuno. Resulta sorprendente que Neptuno pudiese desestabilizar objetos que pasan a una distancia de 5-8 UA, lo cual a corresponde a $\sim 10$ veces el radio de la esfera de influencia gravitacional de dicho planeta. Es importante destacar que la inestabilidad no se debe a encuentros cercanos con Neptuno, sino a la superposición de sus resonancias de movimientos medios exteriores. Cuando un objeto se encuentran dentro de lo límites de superposición de resonancias, su semieje mayor evoluciona por medio de saltos, pasando desde la vecindad de una resonancia a otra, fundamentalmente durante una conjunción con el planeta. Dado que la órbita de Neptuno es pequeña, el parámetro de Tisserand, dado por

$$
T=\frac{a_{\mathrm{N}}}{a}+2 \sqrt{\left(1-e^{2}\right) \frac{a}{a_{\mathrm{N}}}} \cos i
$$

es aproximadamente conservado, donde $a$, e e $i$ son el semieje mayor, la excentricidad y la inclinación del objeto, y $a_{\mathrm{N}}$ es el semieje mayor de Neptuno. De este modo, la excentricidad experimenta variaciones correlacionadas con el semieje mayor, mientras que la distancia perihélica se mantiene aproximadamente constante. Como consecuencia, la evolución del objeto en el plano $(a, e)$ deriva en su inclusión dentro del Disco Dispersado. El límite de esta estructura está dado por la transición entre la regiones obscura y de color de la Figura 3.5.

Tal como se aprecia de la Figura 3.5, no todos los cuerpos con distancias perihélicas $q \lesssim 35$ UA resultan ser inestables. En efecto, aquellos cuerpos que residen dentro de resonancias de movimientos medios con Neptuno de bajo orden muestran un comportamiento estable. Dichos objetos, a pesar de aproximarse a la órbita de Neptuno en su pasaje por el perihelio, nunca sufren un encuentro cercano con dicho planeta. Como dijimos en la Sección 3.1.1, la resonancia actúa como un mecanismo de protección para sus objetos residentes, evitando las sucesivas perturbaciones que Neptuno podría generar en un encuentro cercano. La representación de los Plutinos, a $\sim 39.5 \mathrm{UA}$, pone en evidencia que la resonancia 3:2 resulta ser la más densamente poblada de la Región Transneptuniana.

Las resonancias de movimientos medios con Neptuno de bajo orden no representan los únicos agentes relevantes que estructuran la dinámica de la Región Transneptuniana. En efecto, las resonancias seculares también juegan un rol fundamental. Knezevic et al. (1991) computaron la localización de las resonancias seculares en el Sistema Solar, desde las 2 hasta las 50 UA. Ellos mostraron que las resonancias seculares que involucran tanto a la longitud del perihelio como a la longitud del nodo de Neptuno están presentes en la región de semiejes $40<a<42$ UA para inclinaciones $i<15^{\circ}$. Debido a esto, los objetos confinados en dicha región sobre órbitas de baja inclinación resultan ser inestables mientras que aquellos con inclinaciones mayores muestran un comportamiento estable. En efecto, la Figura 3.5 confirma este punto, mostrando que muchos objetos con inclinaciones $i>4^{\circ}$ (puntos violetas) están presentes en la región de semiejes $40<a<42$ UA mientras que aquellos con inclinaciones menores (puntos verdes) están ausentes.

Otra característica importante revelada por la Figura 3.5 es la presencia de finas bandas de color marrón, las cuales representan regiones donde los objetos llegan a cruzar la órbita 


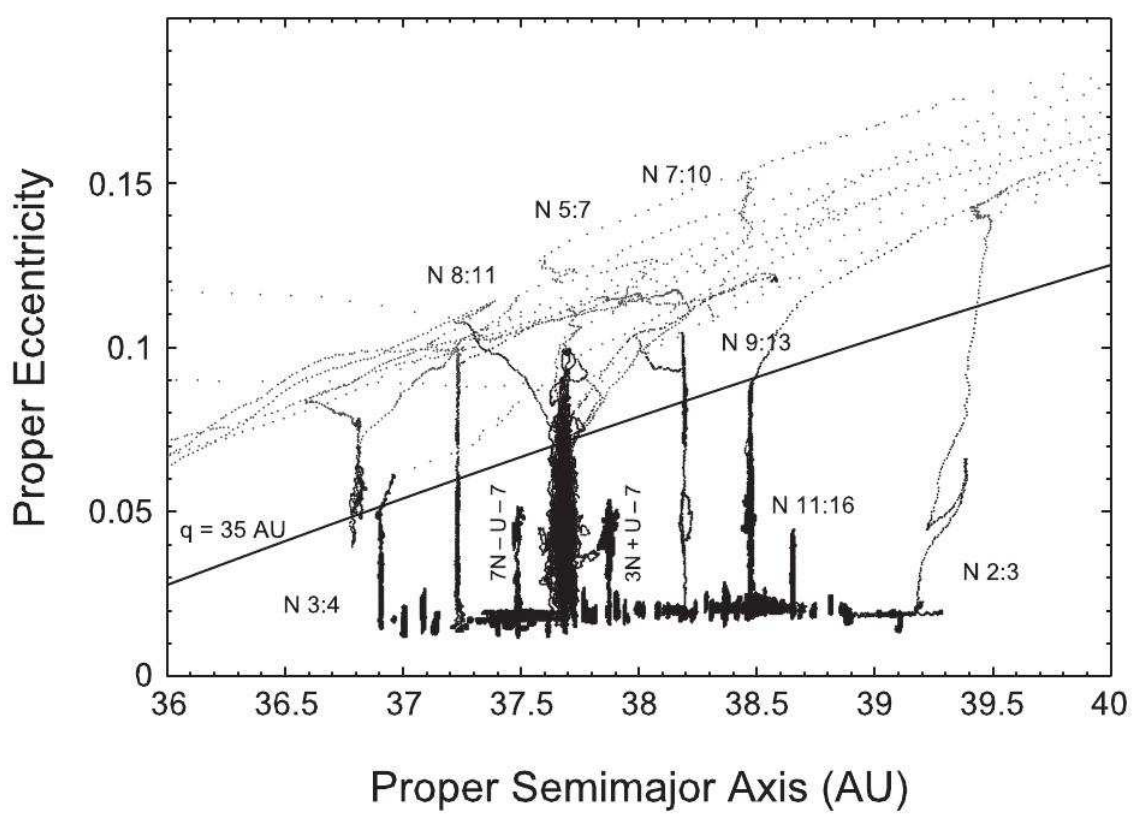

Figura 3.6. Difusión caótica en la Región Transneptuniana. Evolución de objetos con semiejes mayores iniciales en el rango $36.5<a<39.5 \mathrm{UA}$, y excentricidades iniciales $e=0,015$. Los puntos representan el camino evolutivo de las partículas sobre la edad del Sistema Solar, en el espacio de los elementos propios. Los puntos grises nos muestran la evolución de los objetos después de alcanzar una distancia perihélica $q<32$ UA por primera vez. Diversas resonancias de movimientos medios con Neptuno, y resonancias de tres cuerpos con Urano y Neptuno pueden ser observadas. Figura extraída de Nesvorný \& Roig (2001).

de Neptuno después de miles de millones de años de evolución. Si bien por muchos años la naturaleza de estas órbitas constituyó un tema de debate, Nesvorný \& Roig (2000) encontraron que estas regiones débilmente inestables están generalmente asociadas con resonancias de movimientos medios con Neptuno de alto orden o resonancias de tres cuerpos con Urano y Neptuno.

Debido a la excentricidad no nula de las órbitas planetarias, el comportamiento dinámico en estas resonancias resulta ser caótico. Mientras que el semieje mayor de los objetos de prueba permanecen en el valor resonante correspondiente, las excentricidades orbitales evolucionan lentamente, describiendo un trazo vertical en un plano $(a, e)$. Este comportamiento es evidente a partir de la Figura 3.6, extraída del trabajo de Nesvorný \& Roig (2001). Las características mencionadas están asociadas a un proceso de difusión caótica. En este mecanismo, el crecimiento gradual de la excentricidad puede llevar al decrecimiento de la distancia perihélica de un objeto por debajo de las 35 UA, donde las perturbaciones por parte de Neptuno resultan ser significativas. Tal como dijimos en la Sección 3.1.1, las resonancias de alto orden son demasiado débiles como para proporcionar un mecanismo de protección efectivo contra los encuentros cercanos con Neptuno. De este modo, una vez que la distancia perihélica del objeto decrece por debajo de las $35 \mathrm{UA}$, los encuentros sucesivos con dicho planeta modifican su semieje orbital, removiéndolo de la resonancia para luego evolucionar en el Disco Dispersado. 
Por otra parte, algunas resonancias son tan débiles que si bien incrementan gradualmente las excentricidades de los objetos, los mismos no pueden alcanzar distancias perihélicas menores a 35 UA sobre la edad del Sistema Solar (Figura 3.6). De este modo, si bien estos objetos no son estables desde un punto de vista dinámico, los mismos pueden ser considerados como tales desde la perspectiva astronómica.

Finalmente, debemos notar que la difusión caótica es efectiva sólo para ciertas resonancias. De la Figura 3.6 se observa que muchos de los objetos simulados no están afectados por ninguna difusión macroscópica, preservando sus pequeñas excentricidades iniciales sobre la edad del Sistema Solar.

\section{Estructura Dinámica de la Resonancia 3:2 con Neptuno}

Los objetos que residen en la resonancia de movimientos medios 3:2 con Neptuno reciben el nombre de Plutinos. La dinámica de los Plutinos está caracterizada por la amplitud de libración del argumento resonante $\phi_{3: 2}$ alrededor de $180^{\circ}$, el cual viene dado por la expresión

$$
\phi_{3: 2}=3 \lambda-2 \lambda_{\mathrm{N}}-\bar{\omega},
$$

donde $\lambda_{\mathrm{N}}$ representa la longitud media de Neptuno, y $\lambda$ y $\bar{\omega}$ son la longitud media y la longitud del perihelio de un Plutino, respectivamente.

Tal como hemos discutido en la Sección 3.1.1, la amplitud de libración del argumento resonante resulta ser un indicador importante de la estabilidad en una resonancia. Levison \& Stern (1995) analizaron los límites de estabilidad de la resonancia de movimientos medios 3:2 con Neptuno en función de las amplitudes de libración del argumento resonante $\phi_{3: 2}$ y el argumento del perihelio $\omega^{3}$. Estos autores encontraron que para inclinaciones similares a la de Plutón $\left(\sim 17^{\circ}\right)$, aquellas órbitas que comienzan con una amplitud de libración del argumento resonante $\phi_{3: 2}$ menor a $50^{\circ}$ son estables, mientras que aquellas mayores a $120^{\circ}$ dan lugar a órbitas inestables sobre $4 \times 10^{9}$ años. Además, para valores intermedios de la amplitud de libración del argumento resonante $\phi_{3: 2}$, estos autores indicaron que la estabilidad orbital sólo es posible para valores pequeños de la amplitud de libración del argumento del perihelio $\omega$.

Paralelamente, Duncan et al. (1995) desarrollaron un interesante análisis sobre la estructura dinámica de la Región Transneptuniana. Concerniente a los Plutinos, estos autores indicaron que para excentricidades $e=0,2$, el movimiento resulta ser estable sobre la edad del Sistema Solar para valores de la amplitud de libración del argumento resonante $\phi_{3: 2}$ menores a $70^{\circ}$. Por el contrario, para amplitudes de libración mayores a $120^{\circ}$, el movimiento es completamente inestable y los objetos son removidos de la resonancia sobre cortos intervalos, del orden de $10^{6}-10^{8}$ años. El resultado más importante obtenido por Duncan et al. (1995) es el descubrimiento de objetos que escapan desde la resonancia 3:2 y encuentran a Neptuno sobre escalas de tiempo mayores a $10^{9}$ años. La existencia de este tipo de órbitas resulta ser significativa. Ya que los tiempos de vida dinámicos de los cometas resultan ser muy cortos, aquellos que hoy observamos deben haber abandonado sus regiones fuentes sólo unos pocos millones de años atrás. La existencia de órbitas dinámicamente inestables sobre tiempos comparables a la edad del Sistema Solar en la resonancia 3:2 con Neptuno, llevaron a Duncan et al. (1995) a sugerir una posible conexión entre los Plutinos y la población de Cometas de la Familia de Júpiter.

\footnotetext{
${ }^{3} \omega=\bar{\omega}-\Omega$, siendo $\bar{\omega}$ y $\Omega$ la longitud del perihelio y la longitud del nodo ascendente, respectivamente.
} 


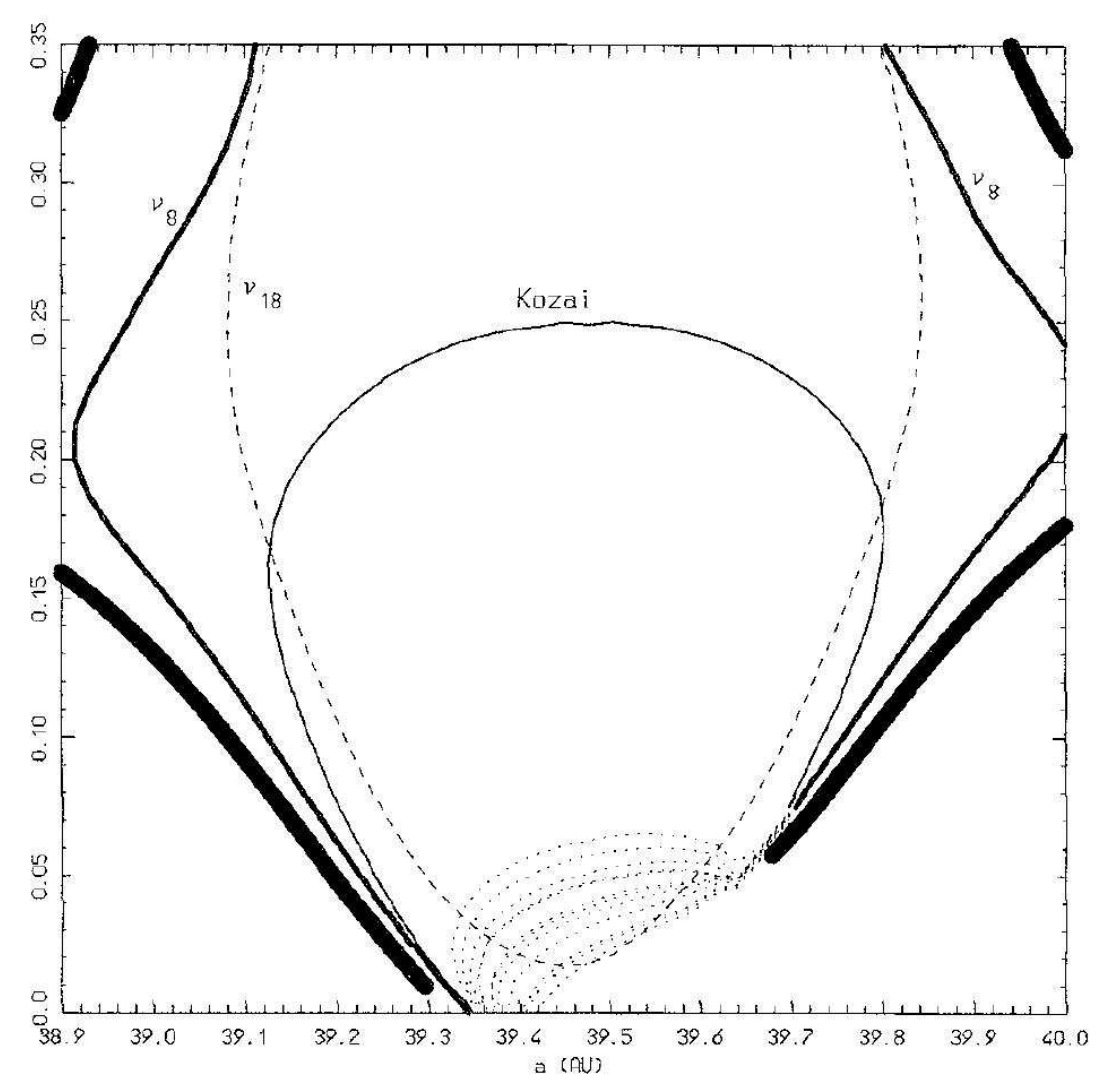

Figura 3.7. Localización de las principales resonancias internas en la conmensurabilidad 3:2 con Neptuno. Las curvas negras más gruesas representan los bordes de la resonancia. Notemos que el ancho de la misma disminuye a grandes excentricidades. Figura extraída de Morbidelli (1997).

Motivado por este resultado, Morbidelli (1997) estudió la estabilidad de la resonancia 3:2 con Neptuno a bajas inclinaciones, analizando el número de objetos que escapan desde la resonancia como consecuencia de la difusión caótica, y su relación con el flujo de Cometas de la Familia de Júpiter. En este trabajo, Morbidelli (1997) confirmó los resultados de Duncan et al. (1995), encontrando una región débilmente difusiva a moderadas amplitudes de libración, capaz de remover objetos sobre tiempos comparables a la edad del Sistema Solar.

La Figura 3.7 muestra la localización de las principales resonancias seculares dentro de la conmensurabilidad de movimientos medios 3:2 con Neptuno. La resonancia secular $\nu_{8}$ se produce debido a una conmensurabilidad 1:1 entre la tasa de precesión de la longitud del perihelio de un objeto y la tasa de precesión media de la longitud del perihelio de Neptuno. Por otra parte, la resonancia secular $\nu_{18}$ corresponde a una conmensurabilidad 1:1 entre la tasa de precesión de la longitud del nodo de un objeto y la tasa de precesión media de la longitud del nodo de Neptuno. Finalmente, la resonancia de Kozai se produce debido a una conmensurabilidad 1:1 entra las tasas de precesión que involucran las longitudes del perihelio y del nodo de un objeto. Tal como se describió en la Sección 3.1.1, $\nu_{8}$ excita las excentricidades, $\nu_{18}$ afecta las inclinaciones, mientras que la resonancia de Kozai puede modificar ambos parámetros orbitales, aunque su efectividad se incrementa sobre objetos de elevada inclina- 


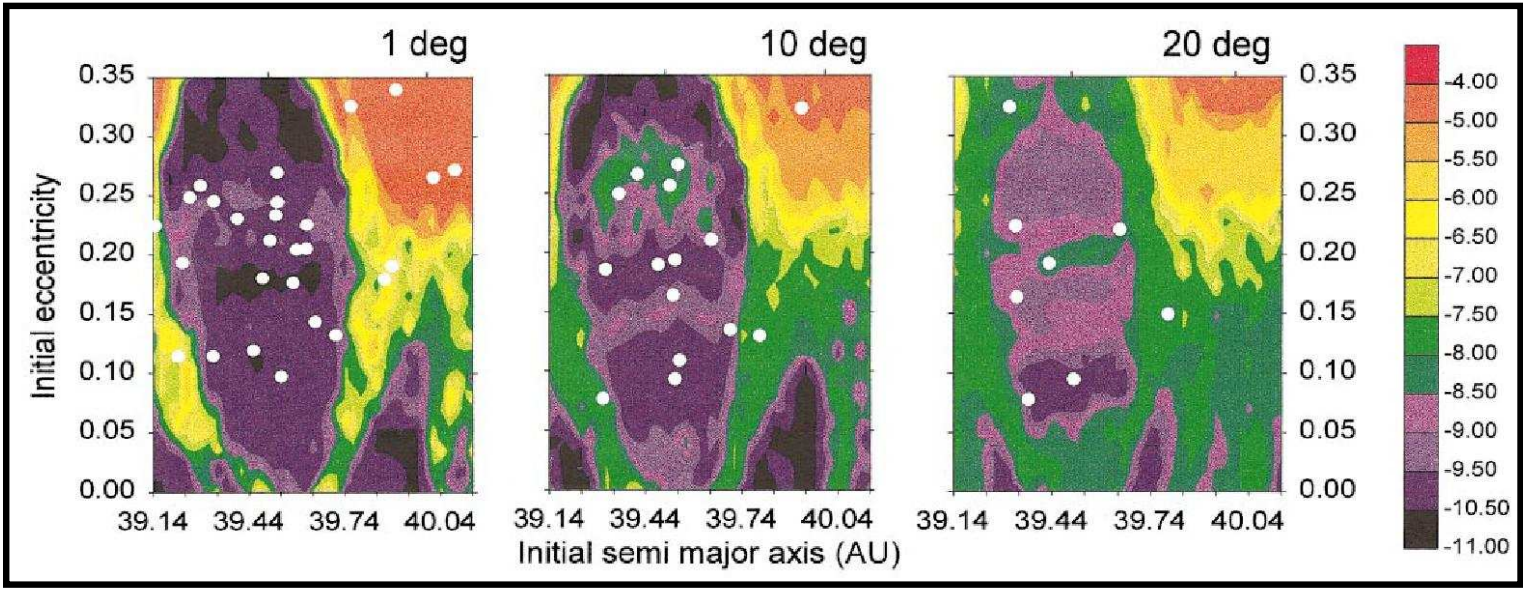

Figura 3.8. Mapas de estabilidad de la resonancia 3:2 con Neptuno. A la derecha de la figura, los colores asociados a valores $\lesssim-9.39$ representan regiones estables sobre la edad del Sistema Solar. Notemos como el tamaño de la región de estabilidad presente en la zona central de la resonancia, decrece con un aumento de la inclinación, lo cual es debido a la acción de una resonancia de Kozai. Los puntos blancos indican la localización de los Plutinos observados. Figura extraída de Melita \& Brunini (2000).

ción. Otro rasgo distintivo observado de la Figura 3.7 es la presencia de un gran número de resonancias secundarias de bajo orden a pequeñas excentricidades.

Con el objetivo de realizar un estudio comparativo entre las principales resonancias de movimientos medios de la Región Transneptuniana, Melita \& Brunini (2000) analizaron la estructura dinámica de la resonancia de movimientos medios 3:2 con Neptuno. Estos autores desarrollaron mapas de estabilidad de esta región resonante en el plano $(a, e)$, para tres inclinaciones iniciales diferentes. Dichos mapas pueden ser observados en la Figura 3.8. Los colores son indicativos de la estabilidad en la resonancia. En efecto, de acuerdo a sus resultados y observando la escala a la derecha del gráfico, los colores asociados a valores $\lesssim-9.39$ representan regiones estables sobre la edad del Sistema Solar.

Las principales características observadas en estos mapas pueden ser explicadas a través de la acción de las resonancias internas a la conmensurabilidad 3:2 con Neptuno, mostradas en la Figura 3.7. En efecto, observando ambas figuras es evidente que la región inestable a bajas excentricidades corresponde a la región caótica originada por la superposición de resonancias secundarias. Por otra parte, a bajas y moderadas excentricidades, la fuerte región de inestabilidad asociada a la vecindad de los bordes de la resonancia se debe fundamentalmente a la superposición de la resonancia secular $\nu_{18}$ con la resonancia de Kozai. A excentricidades más elevadas, los límites de las regiones inestables parecen estar delineados por la resonancia secular $\nu_{8}$.

Por otra parte, a bajas inclinaciones, la zona central de la resonancia representa una región estable muy robusta. Sin embargo, para inclinaciones más elevadas, el centro de la resonancia se muestra más inestable. Esto se debe a la acción de la resonancia de Kozai, que como ya indicamos, resulta ser más eficiente para inclinaciones orbitales grandes.

La Figura 3.8 ilustra también la localización de los Plutinos observados hasta el año 2000. De la misma se observa que la mayoría de ellos se ubican en las regiones estables de la 
resonancia, mientras que sólo unos pocos están asociados a zonas inestables. Melita \& Brunini (2000) argumentaron que estos últimos podrían estar pasando por un estado de transición provenientes de otras regiones, o bien ser objetos eyectados de las zonas de estabilidad debido a colisiones o encuentros gravitacionales, o podrían tener órbitas pobremente determinadas y ubicarse realmente en alguna región estable del espacio de las fases.

\section{Propiedades Primordiales de la Región Transneptuniana}

Siguiendo los análisis concernientes a la estructura dinámica actual de la Región Transneptuniana, es posible divisar ciertas propiedades que deben ser explicadas necesariamente dentro del marco de trabajo del proceso de formación y evolución primordial del Sistema Solar. De acuerdo a Morbidelli et al. (2008), dichas propiedades son las siguientes:

1. La existencia de numerosas poblaciones de objetos en las principales resonancias de movimientos medios con Neptuno. Los Objetos Resonantes muestran un amplio rango de excentricidades e inclinaciones. Sin embargo, las resonancias son regiones dinámicamente estables y por ende, las mismas no nos permiten explicar los más elevados valores asociados a estos parámetros orbitales.

2. La excitación de las excentricidades en el cinturón clásico. La excentricidad media de los objetos del cinturón clásico es de $\sim 0.07$. Aun cuando la excentricidad media actual de los objetos de esta población es relativamente pequeña, la misma es, sin embargo, un orden de magnitud (o más) mayor que aquella que debió haber existido en la etapa de formación de los objetos de la Región Transneptuniana. La dinámica actual resulta ser estable en la región del cinturón clásico, de modo que sin mecanismos perturbativos adicionales, las pequeñas excentricidades primordiales deberían haber sido preservadas hasta los tiempos actuales.

3. La distribución peculiar de los objetos del cinturón clásico en el plano (a,e). La población de objetos clásicos sobre órbitas con excentricidades $e \lesssim 0.05$ finaliza a $\sim 44$ UA, mientras que más allá de este límite la excentricidad tiende a incrementarse con el semieje. Si esto fuese simplemente la consecuencia de un sesgo observacional que favorece el descubrimiento de objetos sobre órbitas con distancias perihélicas $q$ bajas, sería razonable que el límite inferior de la distribución de objetos en el plano $(a, e)$ entre las 44 y las 48 UA siguiese una curva de $q$ constante. Sin embargo, esto no es lo que reflejan las observaciones. En efecto, la evidencia observacional indica que la excentricidad de dicho límite crece más fuertemente con el semieje mayor, relativo al valor que tomaría si siguiese una curva de $q$ constante. De este modo, la baja densidad relativa de objetos con pequeñas excentricidades y semiejes mayores entre 44 y 48 UA es probablemente una característica real de la Región Transneptuniana. De acuerdo a la discusión ya realizada, esta baja densidad de objetos no puede ser explicada por la ausencia de estabilidad dinámica en dicha región.

4. El límite exterior del cinturón clásico. Este límite parece estar precisamente en la localización de la resonancia de movimientos medios 2:1 con Neptuno. Sólo objetos con grandes excentricidades, típicos del Disco Dispersado, o aquellos asociados a la población del Disco Dispersado Extendido parecen existir más allá de dicho límite. Nuevamente, la ausencia de objetos con bajas excentricidades más allá de la resonancia 1:2 con Neptuno 
no puede ser explicada como consecuencia de un sesgo observacional (Trujillo \& Brown 2001; Allen et al. 2001, 2002). De acuerdo a los estudios ya discutidos previamente, la región más allá de la resonancia 1:2 con Neptuno resulta ser estable, incluso a moderadas excentricidades. De este modo, una población primordial distante debería haber permanecido allí hasta los tiempos actuales.

5. La distribución de inclinaciones en el cinturón clásico. Tal como ya dijimos, Brown (2001) argumentó que la distribución real de inclinaciones $i$ de los objetos del cinturón clásico es bimodal y puede ser ajustada por la superposición de dos funciones gausianas, una con $\sigma \sim 2^{\circ}$ para $i \lesssim 4^{\circ}$ y otra con $\sigma \sim 12^{\circ}$ para $i \gtrsim 4^{\circ}$. A partir de este trabajo, los objetos clásicos con $i \lesssim 4^{\circ}$ constituyen la población cold, mientras que aquellos con inclinaciones superiores representan la población hot. Los datos observacionales indican que algunos objetos clásicos de la población hot alcanzan inclinaciones muy elevadas, incluso de hasta $30^{\circ}$. Sin embargo, la estructura dinámica actual de la Región Transneptuniana no nos permite explicar la existencia de órbitas con inclinaciones tan elevadas.

6. Correlaciones entre propiedades físicas y parámetros orbitales. De acuerdo al estudio realizado por Levison \& Stern (2001), el agrupamiento de objetos del cinturón clásico visible en el plano $(a, i)$ a bajas inclinaciones desaparece si sólo se seleccionan objetos con magnitudes absolutas $H \lesssim 6$. Este resultado implica que los objetos intrínsecamente brillantes están pobremente representados en la población cold. Por otra parte, Grundy et al. (2005) han mostrado que los objetos de la población cold tienen un albedo medio mayor que aquellos de la población hot. Este resultado junto con la correlación encontrada por Levison \& Stern (2001) nos permite inferir que la población hot contiene objetos más grandes. En efecto, Bernstein et al. (2004) mostraron que la población hot tiene una distribución de magnitudes $H$ que resulta más achatada que aquella asociada a la población cold, lo cual es consistente con la ausencia de objetos grandes en esta última. Por otra parte, Doressoundiran et al. (2007) indicaron que existe una correlación entre el color y la inclinación para los objetos clásicos. Según este análisis, la población hot muestra un amplio rango de colores, desde rojos a grises, mientras que los objetos de la población cold son fundamentalmente rojos. Las diferencias expuestas en las propiedades físicas sugieren que las poblaciones cold y hot tienen distintos orígenes.

7. El déficit de masa de la Región Transneptuniana. La masa actual que alberga la Región Transneptuniana es muy pequeña. Sus estimaciones varían desde $0.01 \mathrm{M}_{\oplus}$ (Bernstein et al. 2004) a $0.1 \mathrm{M}_{\oplus}$ (Gladman et al. 2001). La incerteza en la determinación de este valor se debe fundamentalmente a la conversión desde magnitudes absolutas a tamaños, suposiciones sobre la densidad de masa de los objetos y ambigüedades en la distribución de tamaños. Cualquiera sea la masa total actual de la Región Transneptuniana, los estudios indican que tuvo lugar un déficit significativo de masa de 2 a 3 órdenes de magnitud, con respecto a lo que los modelos indican que debió haber existido en las etapas primitivas para que los objetos de la Región Transneptuniana se formaran in situ. En efecto, para que los objetos que hoy vemos se hayan formado en un tiempo de $10^{7}$ - $10^{8}$ años, la Región Transneptuniana tuvo que contener originalmente de 10 a $30 \mathrm{M}_{\oplus}$ de material sólido en un disco dinámicamente frío (Stern 1996; Stern \& Colwell 1997a, 1997b; Kenyon \& Luu 1998, 1999a, 1999b; Kenyon \& Bromley 2004). Según lo que hemos visto, la mayor parte de la Región Transneptuniana actual es dinámicamente estable y por ende no es posible explicar la remoción de más del $99 \%$ de su masa primordial 
dentro de dicho contexto.

\section{Modelo de Niza}

Basado en los efectos de una inestabilidad dinámica sobre las órbitas de Urano y Neptuno, el Modelo de Niza es el primero en reproducir satisfactoriamente las propiedades orbitales de los planetas exteriores (Tsiganis et al. 2005), la captura de Troyanos de Júpiter (Morbidelli et al. 2005) y Neptuno (Tsiganis et al. 2005; Sheppard \& Trujillo 2006), las principales características del bombardeo tardío sobre los planetas terrestres (Gomes et al. 2005) y las propiedades más intrigantes y relevantes de la Región Transneptuniana (Levison et al. 2007).

En este modelo, los planetas exteriores poseen órbitas iniciales casi circulares y coplanares y constituyen un sistema algo más compacto, con separaciones orbitales que resultan ser significativamente menores que las observadas actualmente. Más precisamente, los planetas exteriores son distribuidos aproximadamente desde las 5.5 hasta las 14 UA, y Saturno es colocado más cerca de Júpiter que su mutua resonancia de movimientos medios 1:2. Más allá de las órbitas de los planetas gigantes, se extiende un disco de planetesimales sobre órbitas cuyo tiempo de vida dinámico es de al menos $3 \times 10^{6}$ años, el cual es el tiempo de vida asumido para la componente gaseosa del disco. El límite exterior del disco de planetesimales se ubica a $\sim 34$ UA y su masa total es de $\sim 35 \mathrm{M}_{\oplus}$.

Con esta configuración, los planetesimales ubicados en el límite interior del disco son dispersados por Neptuno sobre una escala de tiempo de unos pocos millones de años. Como consecuencia, la migración de los planetas gigantes se produce a una tasa muy lenta ya que la misma está gobernada por la lenta tasa de escape de planetesimales desde el disco. Debido a que el sistema planetario sería estable en ausencia de interacciones con los planetesimales, esta lenta migración continua por un largo tiempo, aumentando levemente a medida que las partículas inestables son removidas del sistema. Después de un cierto tiempo, el cual va desde $3.5 \times 10^{8}$ a $1.1 \times 10^{9}$ años en las simulaciones de Gomes et al. (2005), Júpiter y Saturno cruzan su mutua resonancia de movimientos medios 1:2, lo cual excita sus excentricidades a valores levemente mayores que aquellos que poseen actualmente. Sin embargo, este evento conduce a un aumento significativo en las excentricidades de Urano y Neptuno. En efecto, las órbitas de estos planetas llegan a ser caóticas y comienzan a aproximarse. De este modo, el evento de cruce de la resonancia es seguido por una fase corta de encuentros. Como consecuencia, Urano y Neptuno son dispersados hacia afuera, sobre órbitas con grandes excentricidades (esto es, $e \sim 0.3-0.4$ ), las cuales penetran profundamente en el disco. Este evento desestabiliza el disco completo de planetesimales, los cuales son dispersados sobre todo el Sistema Solar. Las excentricidades de los planetas gigantes son amortiguadas sobre una escala de tiempo de unos pocos millones de años, debido a la fricción dinámica ejercida por los planetesimales. De este modo, los planetas se desacoplan unos de otros y la fase de mutuos encuentros rápidamente finaliza. Durante y después de la fase de amortiguamiento de las excentricidades, los planetas gigantes continúan su migración radial y eventualmente alcanzan sus órbitas finales cuando la mayor parte del disco ha sido eliminado.

Levison et al. (2007) analizaron este proceso a partir de simulaciones numéricas. Los resultados de tales simulaciones basadas en el Modelo de Niza han sido capaces de reproducir las principales características dinámicas de la Región Transneptuniana, entre las cuales destacamos: 
1. La población de objetos sobre órbitas casi circulares que se extiende hasta $\sim 45 \mathrm{UA}$.

2. El déficit de objetos sobre órbitas con baja excentricidad entre 45 y 48 UA.

3. La localización del límite exterior del cinturón clásico en la resonancia de movimientos medios 1:2 con Neptuno.

4. La existencia de una población de objetos más allá de la resonancia 1:2 con distancias perihélicas $q \sim 40 \mathrm{UA}$.

5. La distribución de inclinaciones de la población cold del cinturón clásico.

6. La distribución orbital de las poblaciones asociadas con las principales resonancias de movimientos medios con Neptuno.

Además, el Modelo de Niza ha permitido explicar:

1. El déficit de masa de la Región Transneptuniana. Las simulaciones de Levison et al. (2007) muestran que entre 50 y 130 partículas del total de 60000 que inicialmente constituyen el disco de planetesimales son atrapadas en el cinturón clásico. De acuerdo al Modelo de Niza, el disco de planetesimales comienza con $35 \mathrm{M}_{\oplus}$ de material, por lo que el cinturón clásico debería contener actualmente entre $\sim 0.02 \mathrm{y} \sim 0.08 \mathrm{M}_{\oplus}$, en un buen acuerdo con las estimaciones observacionales.

2. Las correlaciones observadas entre las propiedades físicas y la inclinación de los objetos en el cinturón clásico. En efecto, las simulaciones numéricas de Levison et al. (2007) indican que la mayoría de las partículas que son atrapadas en la población cold del cinturón clásico provienen de las partes más externas del disco de planetesimales, particularmente más allá de las 29 UA. De modo contrario, las simulaciones muestran que una fracción significativa que las partículas atrapadas en la población hot tienen su origen en el disco interior. De este modo, si se asume que los objetos más grandes pudieron formarse sólo en la parte interna del disco de planetesimales, los mismos serán encontrados preferentemente en la población hot. Del mismo modo, si se asume (aunque por alguna razón desconocida) que los objetos de la parte exterior del disco son rojos y aquellos de la parte interna son grises, la población cold del cinturón clásico estaría compuesta casi exclusivamente de objetos rojos, mientras que la población hot contendría una mezcla de ambos.

3. La ausencia de correlación entre el color y la inclinación de los Plutinos. En efecto, las simulaciones de Levison et al. (2007) indican que la población de Plutinos está constituida por partículas provenientes de las partes internas y externas del disco de planetesimales, las cuales se mezclan muy bien en el plano excentricidad.vs.inclinación. Esta ausencia de correlación entre el color y la inclinación de los Plutinos es consistente con estudios observacionales realizados por Peixinho et al. (2004) y Doressoundiram et al. (2005).

El Modelo de Niza tiene dos falencias que deben ser mencionadas. En efecto, las simulaciones numéricas basadas en el Modelo de Niza desarrolladas hasta aquí no han sido capaces de reproducir de manera simultánea la baja excentricidad media y las más altas inclinaciones 
de la población del cinturón clásico. La excentricidad media de los objetos observados con semiejes mayores entre 42 y 48 UA y distancias perihélicas mayores a 37 UA es de 0.07 , mientras que las simulaciones de Levison et al. (2007) producen un valor de 0.1. Por otra parte, si bien estas simulaciones reproducen la distribución de inclinaciones de la población hot del cinturón clásico entre los $4^{\circ}$ y $10^{\circ}$, existe un déficit de objetos con inclinaciones más elevadas. Los autores indican que estas discrepancias entre las simulaciones numéricas y las observaciones deben ser debidas a simplificaciones del modelo. En efecto, el Modelo de Niza no considera colisiones ni encuentros gravitacionales entre los planetesimales del disco, lo cual podría afectar los resultados. Más allá de estas falencias, el Modelo de Niza representa el escenario más eficaz para explicar de manera conjunta las características orbitales de los planetas gigantes, los Troyanos de Júpiter y Neptuno, el bombardeo tardío sobre los planetas terrestres y las más intrigantes propiedades de la Región Transneptuniana.

\subsubsection{Cometas}

Históricamente, la clasificación de los cometas ha estado basada en sus períodos orbitales, pudiéndose distinguir dos grandes grupos:

1. cometas de largo período, con $P>200$ años, $\mathrm{y}$

2. cometas de corto período, con $P<200$ años. Los miembros de este grupo son subdivididos a su vez en cometas de la familia de Júpiter, con $P<20$ años, y cometas tipo Halley, con $20<P<200$ años.

Levison \& Duncan (1994) desarrollaron simulaciones numéricas de todos los cometas de corto período conocidos, y observaron que la mayoría de los mismos se mueven entre cometas de la familia de Júpiter y cometas tipo Halley muchas veces durante sus tiempos de vida dinámicos. Sin embargo, Levison \& Duncan (1994) encontraron también que el parámetro de Tisserand con respecto a Júpiter, dado por

$$
T=\frac{a_{\mathrm{J}}}{a}+2 \sqrt{\left(1-e^{2}\right) \frac{a}{a_{\mathrm{J}}}} \cos i
$$

no varía significativamente durante sus integraciones, donde $a$, e e $i$ son el semieje mayor, la excentricidad y la inclinación del cometa y $a_{\mathrm{J}}$ es el semieje mayor de Júpiter. Tal como fue propuesto por primera vez por Carusi \& Valsecchi (1987), estos argumentos sugieren que un esquema de clasificación de cometas basado en el parámetro de Tisserand sería mucho más apropiado que aquel basado en el período orbital. De este modo, de acuerdo a su parámetro de Tisserand $T$, los cometas pueden ser clasificados de la siguiente manera (Levison 1996):

1. cometas casi isotrópicos, con $T<2, \mathrm{y}$

2. cometas eclípticos, con $T>2$. Los miembros de este grupo son subdivididos a su vez en tres clases diferenciadas:

- cometas de la familia de Júpiter, con $2<T<3$, los cuales experimentan encuentros a baja velocidad con Júpiter y de este modo pueden ser dominados dinámicamente por el planeta. Este grupo tiene prácticamente los mismos miembros que aquel que reune a los cometas de la familia de Júpiter definidos a partir de sus períodos orbitales; 
- cometas tipo Encke, con $T>3$ y $a<a_{\mathrm{J}}$, por lo cual tienen órbitas completamente interiores a Júpiter;

- cometas tipo Chiron, con $T>3$ y $a>a_{\mathrm{J}}$, los cuales probablemente son equivalentes a los Centauros, definidos en la siguiente sección.

Las características orbitales de los cometas casi isotrópicos y eclípticos sugieren que los mismos provienen desde diferentes fuentes. En efecto, tal como fue discutido en la Sección 2.3, los cometas casi isotrópicos provienen de la Nube de Oort mientras que los cometas eclípticos tienen como fuente a la Región Transneptuniana, particularmente al Disco Dispersado (Duncan \& Levison 1997).

\subsubsection{Centauros}

No existe una definición universalmente aceptada para un Centauro. Es posible encontrar definiciones alternativas en función de sus valores del semieje mayor $a$, de la distancia perihélica $q$ o del parámetro de Tisserand $T$. En efecto, un Centauro puede ser definido como un objeto con un semieje mayor $a$ entre 5 y $30 \mathrm{UA}$, o con una distancia perihélica en el mismo rango de valores o cuyo parámetro de Tisserand $T>3$ y $a>a_{\mathrm{J}}$, siendo $a_{\mathrm{J}}$ el semieje mayor de Júpiter. Más allá de estas discrepancias, es ampliamente aceptado que los Centauros son objetos en un estado transitorio, los cuales entran en la región de los planetas terrestres provenientes de la Región Transneptuniana y evolucionan hacia la zona de los cometas de la familia de Júpiter (Fernández 1980; Duncan et al. 1988; Levison \& Duncan 1997). Las Figuras 3.4a y b muestran la distribución de los Centauros observados hasta la fecha en los planos $(a, e)$ y $(a, i)$, respectivamente. Para realizar dicho gráfico hemos adoptado la definición de Centauro que involucra la distancia perihélica.

La fuente principal de los Centauros es el Disco Dispersado de la Región Transneptuniana. Los objetos del Disco Dispersado son capaces de evolucionar hacia la región de los planetas terrestres como consecuencia de encuentros con Neptuno, llegando a ser Centauros. Di Sisto \& Brunini (2007) estudiaron la población de Centauros a través de simulaciones numéricas, teniendo en cuenta la contribución del disco dispersado. Estos autores encontraron que los objetos del Disco Dispersado con $q<35$ UA son los más eficientes en alcanzar la región de Centauros. Además, la mayoría de estos objetos tienen tiempos de vida entre $10^{7}$ y $10^{8}$ años como Centauros, con un valor medio de $7.2 \times 10^{7}$ años. Un resultado interesante es que el tiempo de vida medio de los Centauros depende de su inclinación inicial $i_{\mathrm{i}}$, alcanzando valores de $4.2 \times 10^{7}$ y $1.9 \times 10^{8}$ años para $i_{\mathrm{i}} \sim 16^{\circ}$ y $\sim 35^{\circ}$, respectivamente. Esto nos indica que los objetos del Disco Dispersado con inclinaciones más elevadas producirán Centauros con tiempos de vida dinámicos mayores.

\subsection{Propiedades Físicas}

En esta sección, describimos las características físicas más importantes asociadas a la población del Cinturón Principal de asteroides, NEOs, Troyanos jovianos y objetos de la Región Transneptuniana. 


\subsubsection{Asteroides del Cinturón Principal y NEOs}

\section{Taxonomía y Albedo}

La base fundamental para llevar a cabo una clasificación taxonómica de los asteroides es el conocimiento del albedo geométrico y los espectros de reflexión en longitudes de onda visibles y del infrarrojo cercano.

El albedo geométrico $A$ es una cantidad representativa de la fracción de luz reflejada por un objeto. De modo más riguroso, el albedo geométrico se define como el cociente entre el brillo de un objeto a ángulo de fase cero y el brillo de un disco perfectamente difusor con un radio igual al del objeto y ubicado a la misma distancia del Sol.

Por otra parte, los espectros de reflexión, los cuales caracterizan las propiedades superficiales de los asteroides, son tomados a longitudes de onda visibles y del infrarrojo cercano. Una de las razones de esto, es que la radiación del Sol tiene un máximo en la región visible media del espectro. Como consecuencia, si bien los objetos iluminados por el Sol tendrán un flujo incidente máximo en esta longitud de onda, la radiación emitida por ellos presentará un máximo dependiente de sus temperaturas superficiales. Para los asteroides, el máximo de la radiación emitida se produce entre los 0.3 y $1.1 \mu \mathrm{m}$, lo cual depende del albedo, la emisividad y las características de los procesos térmicos. Por otra parte, la atmósfera resulta ser relativamente transparente en estas longitudes de onda, comparada con su comportamiento para longitudes de onda ultravioletas o del infrarrojo medio.

La clasificación de los asteroides derivada a partir del albedo geométrico y de los espectros de reflexión da lugar a lo que se conoce como taxonomía de asteroides. Si bien se han propuesto diferentes sistemas de clasificación taxonómicas, el más importante ha sido aquel desarrollado por Tholen (1984), el cual brindó las bases para los estudios futuros. Este esquema de clasificación de taxonomías define 14 clases principales, las cuales son denotadas por las letras $A, B, C, D, E, F, G, M, P, Q, R, S, T$ y $V$. Para algunas de estas clases, la letra que las define está asociada a la composición específica de los objetos que representan. Este es el caso de la clase $S$, que incluye asteroides compuestos por silicatos, la clase $C$, compuestos de carbono y la clase $M$, constituidos por metales, en particular, hierro y níquel.

Estas clases taxonómicas presentan características distintivas en lo que concierne a propiedades espectrales, albedos y composición. La clase $B$ tiene albedos bajos entre el rango 0.04 y 0.08 , mientras que la $C$ muestra albedos entre 0.04 a 0.06 . Las clases $E, M$ y $G$ muestran espectros similares a la clase $C$, aunque albedos más altos de $0.38,0.12$ y 0.09 , respectivamente. Los asteroides $D$ tienen albedos entre 0.04 y 0.07 , mientras que los de la clase $S$, entre 0.14 y 0.17 . Las clases $A, Q, R$ y $V$ presentan albedos moderadamente altos, mientras que para las clases $T$ y $P$, los mismos son bajos. Por otra parte, la Tabla 3.2 resume las más importantes características asociadas a las diferentes clases taxonómicas, en lo concerniente a sus espectros y composición.

Estas clases taxonómicas no se encuentran distribuidas uniformemente a lo largo del Cinturón Principal de asteroides, sino que las mismas muestran diferentes abundancias relativas, como una función de la distancia heliocéntrica (Figura 3.9a, extraída de Bell et al. 1989). A principios de los años 80, Gradie \& Tedesco (1982) estudiaron un total de 656 objetos con diámetros $D \gtrsim 50 \mathrm{~km}$, concluyendo que los asteroides tipo $S$ representan la clase más abundante entre las 2.1 y $2.5 \mathrm{UA}$, los asteroides tipo $C$ dominan la zona central entre las 2.5 


\begin{tabular}{|c|c|c|}
\hline $\begin{array}{c}\text { Clases } \\
\text { Taxonómicas }\end{array}$ & Espectro & Mineralogía \\
\hline$A$ & $\begin{array}{l}\text { Pendiente muy abrupta en el } \\
\text { rojo para } \lambda<0.75 \mu \mathrm{m} \text { y fuerte } \\
\text { absorción para } \lambda>0.75 \mu \mathrm{m}\end{array}$ & $\begin{array}{l}\text { Olivino y } \\
\text { Olivino Metálico }\end{array}$ \\
\hline$B$ & $\begin{array}{c}\text { Sin líneas, } \\
\text { con pendiente neutra }\end{array}$ & $\begin{array}{l}\text { Materiales orgánicos y opacos, } \\
\text { silicatos hidratados } \\
\text { y carbono }\end{array}$ \\
\hline$C$ & $\begin{array}{l}\text { Sin líneas, con pendiente neutra } \\
\text { o levemente enrojecida para } \lambda>0.55 \mu \mathrm{m} \\
\text { Absorción débil para } \lambda<0.55 \mu \mathrm{m}\end{array}$ & $\begin{array}{l}\text { Materiales orgánicos y opacos, } \\
\text { silicatos hidratados } \\
\text { y carbono }\end{array}$ \\
\hline$D$ & $\begin{array}{l}\text { Pocas líneas, } \\
\text { pendiente espectral muy } \\
\text { abrupta en el rojo }\end{array}$ & $\begin{array}{l}\text { Materiales orgánicos (Carbono), } \\
\text { silicatos enriquecidos con materiales } \\
\text { orgánicos y hielos }\end{array}$ \\
\hline$E$ & $\begin{array}{c}\text { Pocas líneas, plano y } \\
\text { levemente enrojecido para } \\
0.3<\lambda<1.1 \mu \mathrm{m}\end{array}$ & $\begin{array}{l}\text { Silicatos libres } \\
\text { de hierro }\end{array}$ \\
\hline$F$ & $\begin{array}{l}\text { Sin líneas, con pendiente neutra } \\
\text { a levemente azulada para } \\
\quad 0.3<\lambda<1.1 \mu \mathrm{m}\end{array}$ & $\begin{array}{l}\text { Materiales orgánicos y opacos, } \\
\text { silicatos hidratados } \\
\text { y carbono }\end{array}$ \\
\hline$G$ & $\begin{array}{c}\text { Sin líneas, pendiente neutra a levemente } \\
\text { enrojecida para } \lambda>0.55 \mu \mathrm{m} \text {. } \\
\text { Fuerte absorción para } \lambda<0.55 \mu \mathrm{m}\end{array}$ & $\begin{array}{l}\text { Materiales orgánicos y opacos, } \\
\text { silicatos hidratados } \\
\text { y carbono }\end{array}$ \\
\hline$M$ & $\begin{array}{l}\text { Pocas líneas, pendiente neutra } \\
\text { a levemente enrojecida para } \\
\qquad 0.3<\lambda<1.1 \mu \mathrm{m}\end{array}$ & $\begin{array}{l}\text { Metales, fundamentalmente } \\
\text { Hierro y Níquel }\end{array}$ \\
\hline$P$ & $\begin{array}{l}\text { Pocas líneas, levemente enrojecido } \\
\qquad 0.3<\lambda<1.1 \mu \mathrm{m}\end{array}$ & $\begin{array}{l}\text { Materiales orgánicos (Carbono), } \\
\text { silicatos enriquecidos con materiales } \\
\text { orgánicos y hielos }\end{array}$ \\
\hline$Q$ & $\begin{array}{l}\text { Pendiente abrupta para } \\
\qquad<0.7 \mu \mathrm{m} . \\
\text { Profunda absorción para } \lambda>0.75 \mu \mathrm{m}\end{array}$ & $\begin{array}{l}\text { Olivinos, } \\
\text { Piroxenos, } \\
\text { Metales }\end{array}$ \\
\hline$R$ & $\begin{array}{l}\text { Pendiente muy abrupta para } \\
\qquad<0.7 \mu \mathrm{m} \text {. } \\
\text { Muy profunda absorción para } \lambda>0.75 \mu \mathrm{m}\end{array}$ & $\begin{array}{l}\text { Olivinos y } \\
\text { Piroxenos }\end{array}$ \\
\hline$S$ & $\begin{array}{l}\text { Absorción fuerte a moderada } \\
\text { para } \lambda<0.7 \mu \mathrm{m}, \text { y de moderada } \\
\text { a inexistente a } \lambda>0.7 \mu \mathrm{m} \text {. }\end{array}$ & $\begin{array}{l}\text { Metales, } \\
\text { Olivinos y } \\
\text { Piroxenos }\end{array}$ \\
\hline$T$ & $\begin{array}{l}\text { Pendiente moderadamente } \\
\text { enrojecida para } \lambda<0.75 \mu \mathrm{m}, \mathrm{y} \\
\text { neutra para } \lambda>0.75 \mu \mathrm{m}\end{array}$ & $\begin{array}{l}\text { Similares a las } \\
\text { clases } C \text { y } D\end{array}$ \\
\hline$V$ & $\begin{array}{l}\text { Pendiente de moderada a muy } \\
\text { abrupta a } \lambda<0.7 \mu \mathrm{m} . \\
\text { Absorción profunda a } \lambda>0.75 \mu \mathrm{m}\end{array}$ & $\begin{array}{l}\text { Piroxenos y } \\
\text { Feldespatos }\end{array}$ \\
\hline
\end{tabular}

Tabla 3.2. Clases taxonómicas de asteroides definidas por Tholen et al. (1984). Características espectrales y mineralógicas. 
y 3.2 UA, mientras que los asteroides tipo $\mathrm{D} / \mathrm{P}$ son las clases dominantes más allá de las 3.2 UA. Además, Gradie \& Tedesco (1982) mostraron la existencia de algunos asteroides tipo $C$ y $D$ entre las 2.1 y 2.5 UA y algunos asteroides tipo $S$ más allá de las 3.2 UA.

Por otra parte, de acuerdo a lo sugerido por Bell (1986), las clases taxonómicas de Tholen pueden ser agrupadas en tres grandes asociaciones llamadas "superclases". Este sistema clasifica a los asteroides de acuerdo al grado de metamorfismo por calentamiento que ellos han experimentado, más que por su composición. Las tres superclases están dadas por: 1) los objetos primitivos, los cuales no han sufrido calentamiento, o al menos sólo un poco, 2) los objetos metamórficos, los cuales han experimentado el suficiente calentamiento como para evidenciar cambios espectrales, y 3) los objetos ígneos, cuyas superficies mineralógicas se formaron a partir de la fusión de materiales. Según lo analizado por Bell (1986), los objetos primitivos están representados por las clases taxonómicas $D, P, C$ y $Q$, los objetos metamórficos por las clases $T, B, G$ y $F$, mientras que los objetos ígneos reunen a las clases $V, R, S, A, M$ y E. La Figura 3.9b, extraída de Bell et al. (1989), nos muestra la distribución de estas tres superclases en el Cinturón Principal de asteroides. A partir de la misma se observa que los objetos ígneos dominan la región interior a $2.7 \mathrm{UA}$, los objetos metamórficos se ubican en una zona alrededor de las 3 UA, mientras que los primitivos dominan la región exterior a 3.4 UA.

Hace unos años, Mothé-Diniz et al. (2003) desarrollaron un análisis destinado a refinar la distribución heliocéntrica de las clases taxonómicas en el Cinturón Principal de asteroides. Usando un total de 2026 objetos con diámetros $D \gtrsim 13 \mathrm{~km}$, ellos encontraron importantes diferencias con el análisis realizado por Gradie \& Tedesco (1982) y otros trabajos previos. En efecto, Mothé-Diniz et al. (2003) concluyeron que los asteroides tipo S representan una fracción significativa de la población del Cinturón Principal más allá de las 3 UA. Además, ellos mostraron discrepancias relevantes en la distribución de clases taxonómicas considerando diferentes rangos de excentricidades e inclinaciones. Estos autores sugieren que las diferencias entre sus resultados y aquellos obtenidos en los trabajos previos son debidas a un incremento en el tamaño de su muestra de asteroides, la cual incluye un número grande de objetos pequeños.

Una evidencia directa de la composición de los asteroides está dada por los meteoritos. En efecto, la gran mayoría de los objetos que impactan la superficie de la Tierra provenientes del espacio son fragmentos resultantes de colisiones entre asteroides. De este modo, si comparamos los espectros de los asteroides con la composición de los meteoritos, es posible encontrar una relación entre estos últimos y las clases taxonómicas. Sin embargo, debemos tener en cuenta que los espectros de los asteroides son representativos de una superficie que ha estado fuertemente afectada por miles de millones de años de radiación solar y procesos colisionales. Este detalle puede afectar la comparación con los meteoritos, ya que los mismos guardan información del proceso de formación de sus cuerpos precursores.

\section{Densidad}

La existencia de sistemas binarios en diversas poblaciones de pequeños planetas de nuestro Sistema Solar ha permitido llevar a cabo estimaciones precisas de la masa de los asteroides. Además, si tenemos la masa total y el tamaño de las componentes de un sistema binario, es posible calcular su densidad $\rho$. 


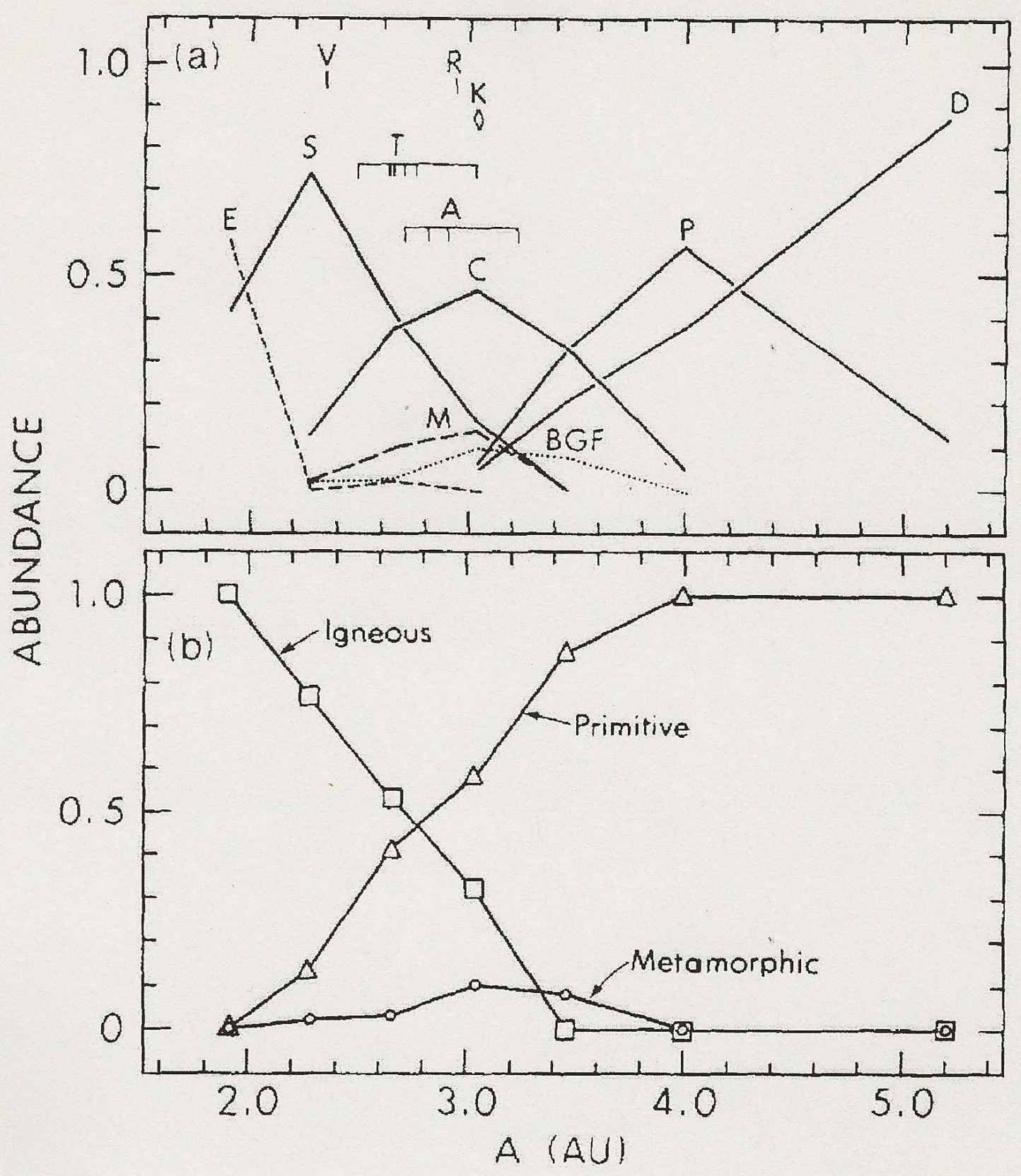

Figura 3.9. Distribución de taxonomías de Tholen (1984) a) y superclases de Bell (1986) b), como función de la distancia heliocéntrica. Figura extraída de Bell et al. (1989) 
Particularmente, las formas irregulares de los NEOs introducen incertezas en la determinación de sus tamaños y por ende, en el cálculo de la densidad. De acuerdo a Noll (2006), la densidad de los NEOs está en el rango $0.8 \leq \rho \leq 2.6 \mathrm{~g} \mathrm{~cm}^{-3}$.

Los asteroides del Cinturón Principal permiten obtener estimaciones más precisas de la densidad. Por un lado, estos objetos pueden ser bien resueltos a partir de imágenes directas o de radar, lo cual conduce a buenas determinaciones de sus diámetros. Por otro lado, muchos de los sistemas binarios del Cinturón Principal son muy asimétricos, teniendo la mayor parte de la masa en la componente primaria. Esta característica hace que las suposiciones realizadas sobre el albedo relativo entre ambas componentes introduzca una incerteza despreciable. De acuerdo a lo sugerido por Noll (2006), la densidad de los asteroides del Cinturón Principal está en el rango $0.6 \leq \rho \leq 3.8 \mathrm{~g} \mathrm{~cm}^{-3}$.

\subsubsection{Troyanos Jovianos}

\section{Taxonomía, Albedo y Color}

La distribuciones de albedos y diámetros de los Troyanos de Júpiter permanecen aun pobremente conocidas. El trabajo más importante desarrollado en este campo fue publicado por Fernández et al. (2003). A partir de observaciones en longitudes de onda visibles y del infrarrojo medio, estos autores derivaron el albedo geométrico y el radio de 32 Troyanos de Júpiter, sin encontrar ninguna correlación estadísticamente significativa entre estos dos parámetros. La Figura 3.10 muestra la distribución de albedos de los Troyanos jovianos derivada por Fernández et al. (2003), junto con aquellas asociadas a cometas activos y candidatos a cometas extintos. De acuerdo a este estudio, el albedo medio de los Troyanos jovianos tiene un valor de $0.041 \pm 0.002$. Además, a partir de la Figura 3.10 resulta evidente que la distribución de albedos de los Troyanos de Júpiter resulta ser consistente con aquella de los cometas, aunque difiere de las distribuciones asociadas a Centauros y objetos de la Región Transneptuniana derivadas por Stansberry et al. (2008). Estos resultados sugieren que las superficies de los Troyanos jovianos son más parecidas a aquellas de los cometas activos y extintos, que a aquellas asociadas a objetos precursores de cometas, tales como los Centauros. Sólo el Troyano joviano (4079) Ennomos presenta un albedo significativamente alto, con un valor de 0.14. Los estudios indican que esta característica peculiar podría ser debida a la presencia de hielo sobre su superficie, excavado desde capas inferiores como consecuencia de un evento de impacto reciente.

Las características superficiales de los Troyanos jovianos conocidos indican que la gran mayoría de los mismos pueden ser clasificados como pertenecientes a la clase $\mathrm{D}$ en la taxonomía de asteroides, aunque las clases C y P también están presentes entre ellos. Este resultado es claramente evidente a partir de la Figura 3.11, la cual es extraída del trabajo de Fornasier et al. (2007). De la misma puede observarse que los asteroides tipo D dominan las poblaciones de Troyanos jovianos asociadas a los puntos $L_{4}$ y $L_{5}$, pero la presencia de asteroides tipo C y $\mathrm{P}$ resulta ser superior en $L_{4}$.

Por otra parte, Fornasier et al. (2007) encontraron una correlación entre el color y la inclinación de los Troyanos jovianos, manifestando que aquellos objetos con inclinaciones orbitales más elevadas resultan ser más rojos que aquellos con inclinaciones menores. Más tarde, Szabó et al. (2007) confirmaron esta tendencia a partir de observaciones realizadas en el marco de trabajo del Sloan Digital Sky Survey. 


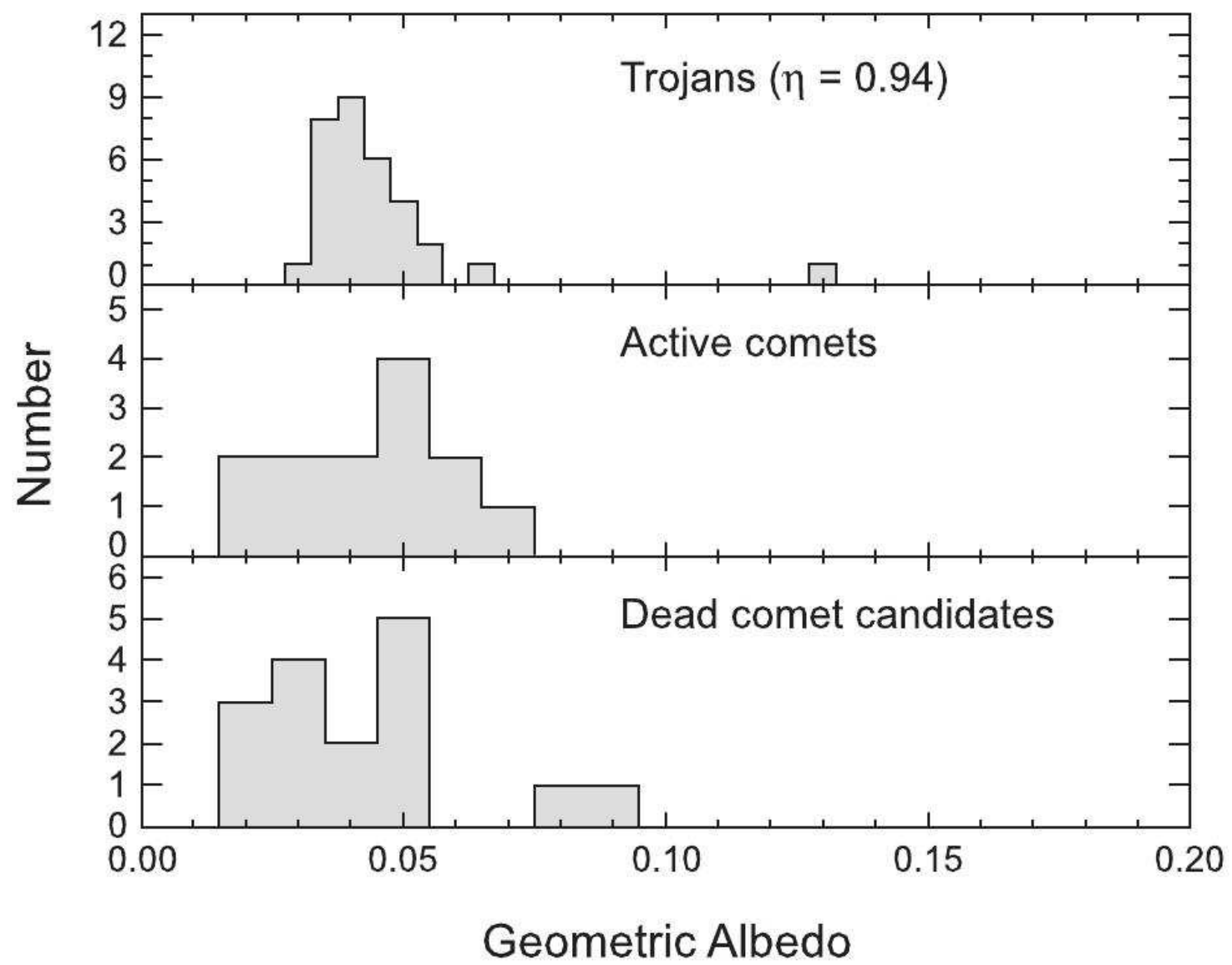

Figura 3.10. Distribución de albedos de Troyanos jovianos derivada por Fernández et al. (2003), junto con aquellas asociadas a cometas activos y candidatos a cometas extintos. El albedo medio de los Troyanos jovianos tiene un valor de $0.041 \pm 0.002$. Figura extraída de Fernández et al. (2003). 

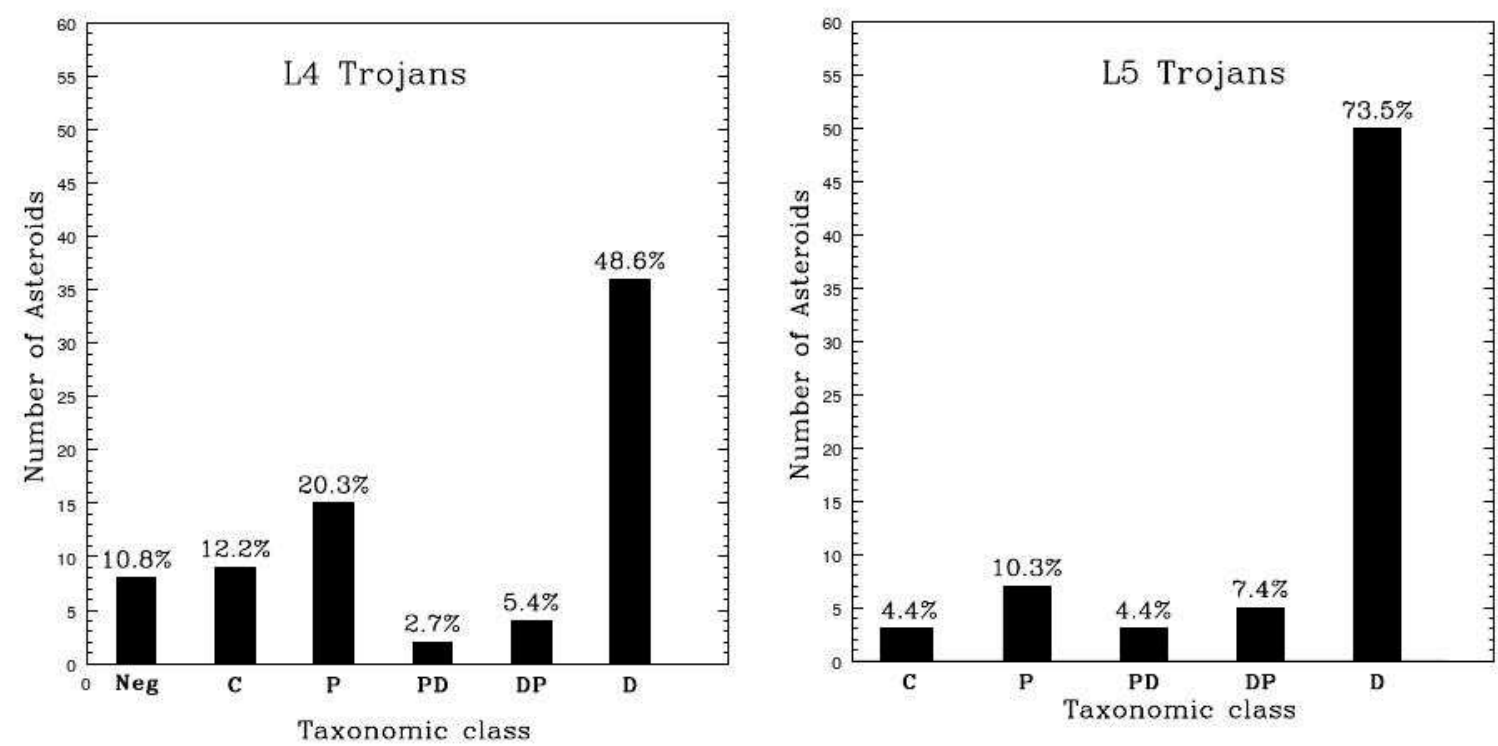

Figura 3.11. Clases taxonómicas entre los Troyanos jovianos. Los asteroides tipo D dominan las poblaciones de Troyanos jovianos asociadas a los puntos $L_{4}$ y $L_{5}$, pero la presencia de asteroides tipo C y P resulta ser superior en $L_{4}$. Figura extraída de Fornasier et al. (2007).

Finalmente, Fornasier et al. (2007) compararon los colores de los Troyanos jovianos con aquellos asociados a las diferentes poblaciones de pequeños cuerpos del Sistema Solar exterior. Ellos encontraron que los Troyanos de Júpiter son los más azules de todas las clases. Además, ellos indicaron que los colores medios de los Troyanos son compatibles con aquellos asociados a los cometas de corto período, aunque sus distribuciones de colores son incompatibles tanto en su ancho como en su forma. Según Fornasier et al. (2007), la compatibilidad en los colores medios de estas poblaciones es causada por el número pequeño de cometas de la muestra más que por una correlación física. Del mismo modo, estos autores encontraron que los colores medios de los Troyanos resultan ser consistentes con aquellos asociados a los Centauros neutros, aunque sus distribuciones son incompatibles. De acuerdo a sus estudios, Fornasier et al. (2007) concluyeron que los Troyanos jovianos presentan características peculiares muy diferentes comparadas con aquellas de las distintas poblaciones de pequeños cuerpos del Sistema Solar exterior. En efecto, estos autores determinaron que nuevos análisis espectroscópicos y polarimétricos resultan ser absolutamente necesarios con el fin de investigar la naturaleza de los Troyanos jovianos y determinar algún tipo de conexión genética entre estos cuerpos y los objetos de la Región Transneptuniana, Centauros y cometas de corto período.

\section{Densidad}

Hasta la fecha, se han realizado estimaciones de la densidad sobre dos Troyanos jovianos únicamente: ellos son (617) Patroclus y (624) Hektor. Patroclus es el primer Troyano binario descubierto (Merline et al. 2001). Determinaciones preliminares de sus parámetros orbitales permitieron obtener una estimación de su densidad, derivando un valor de $\sim 1.3 \mathrm{~g} \mathrm{~cm}^{-3}$ (Merline et al. 2002; Noll 2006), similar a aquellas de los asteroides tipo C. Sin embargo, a partir de observaciones más precisas del período orbital y la separación entre las dos componentes 
del sistema binario, Marchis et al. (2006a) concluyeron que la densidad de Patroclus es de $\sim 0.8 \mathrm{~g} \mathrm{~cm}^{-3}$. Si bien este valor es demasiado bajo comparado con aquel obtenido para los asteroides conocidos, el mismo resulta ser consistente con densidades de $1-2 \mathrm{~g} \mathrm{~cm}^{-3}$ derivadas para objetos de la Región Transneptuniana (Jewitt \& Sheppard 2002; Lacerda \& Luu 2006).

Por otra parte, observaciones realizadas sobre Hektor han revelado que este objeto presenta un pequeño satélite, constituyendo un sistema binario cercano o de contacto (Marchis et al. 2006b). Determinaciones de las masas de las componentes a partir de los parámetros orbitales derivados de las observaciones son consistentes con aquellas obtenidas a partir de modelos de estabilidad de un sistema binario. Estos análisis concuerdan en sugerir que la densidad de Hektor es de $\sim 2.4 \mathrm{~g} \mathrm{~cm}^{-3}$.

Las densidades de los Troyanos jovianos parecen ubicarse en un amplio rango que incluye valores asociados a asteroides del Cinturón Principal (0.6-3.8 $\mathrm{g} \mathrm{cm}^{-3}$ (Noll 2006), núcleos cometarios (0.1-1.5 $\mathrm{g} \mathrm{cm}^{-3}$ (Lowry et al. 2008) y objetos de la Región Transneptuniana (0.5$3 \mathrm{~g} \mathrm{~cm}^{-3}$ (McKinnon et al. 2008, Noll et al. 2008). Este resultado podría indicar que los Troyanos de Júpiter son objetos provenientes de diferentes poblaciones, o bien reflejar los efectos de una actividad colisional significativa. Una consecuencia natural de las colisiones entre asteroides es la formación de estructuras conocidas como rubble piles, los cuales son objetos de muy baja cohesión, constituidos por fragmentos reacumulados. Una población de Troyanos jovianos conformada por una mezcla de tales estructuras y cuerpos primordiales, sería consistente con aquel amplio rango de densidades.

\subsubsection{Centauros y Objetos de la Región Transneptuniana}

\section{Albedo}

Análisis concernientes al albedo geométrico muestran una distinción evidente entre los más grandes miembros de la Región Transneptuniana y el resto de la población (Stansberry et al. 2008). En efecto, debido a la presencia de hielo de $\mathrm{CH}_{4}$ sobre sus superficies, Eris, Make Make y Plutón tienen albedos geométricos extremadamente elevados, cuyos valores son de 0.86, 0.8 y 0.5, respectivamente. Del mismo modo, si bien Haumea no muestra evidencias de $\mathrm{CH}_{4}$, su superficie está dominada por la absorción de hielo de $\mathrm{H}_{2} \mathrm{O}$, dando lugar a un alto albedo geométrico de $\sim 0.73$. Por otra parte, Caronte, el cual presenta un espectro similar, tiene un albedo geométrico de $\sim 0.37$.

La dicotomía existente entre Eris, Make Make, Plutón, Haumea y el resto de los objetos de la Región Transneptuniana y Centauros, en términos de sus albedos y características espectrales, pone en evidencia que ellos son miembros de una clase particular de objetos dentro de esta población. En efecto, con excepción de estos cuerpos peculiares, los valores típicos del albedo geométrico para los objetos de la Región Transneptuniana y Centauros están entre 0.069 y 0.08, con una dispersión de 0.041. Por otra parte, dependiendo de la definición usada para su clasificación, los Centauros parecen tener albedos geométricos levemente menores a aquellos asociados a los objetos objetos transneptunianos.

\section{Taxonomía}

Barucci et al. (2006) propusieron un esquema de clasificación taxonómico para Centauros y objetos de la Región Transneptuniana, basados en las propiedades de sus índices de color. 

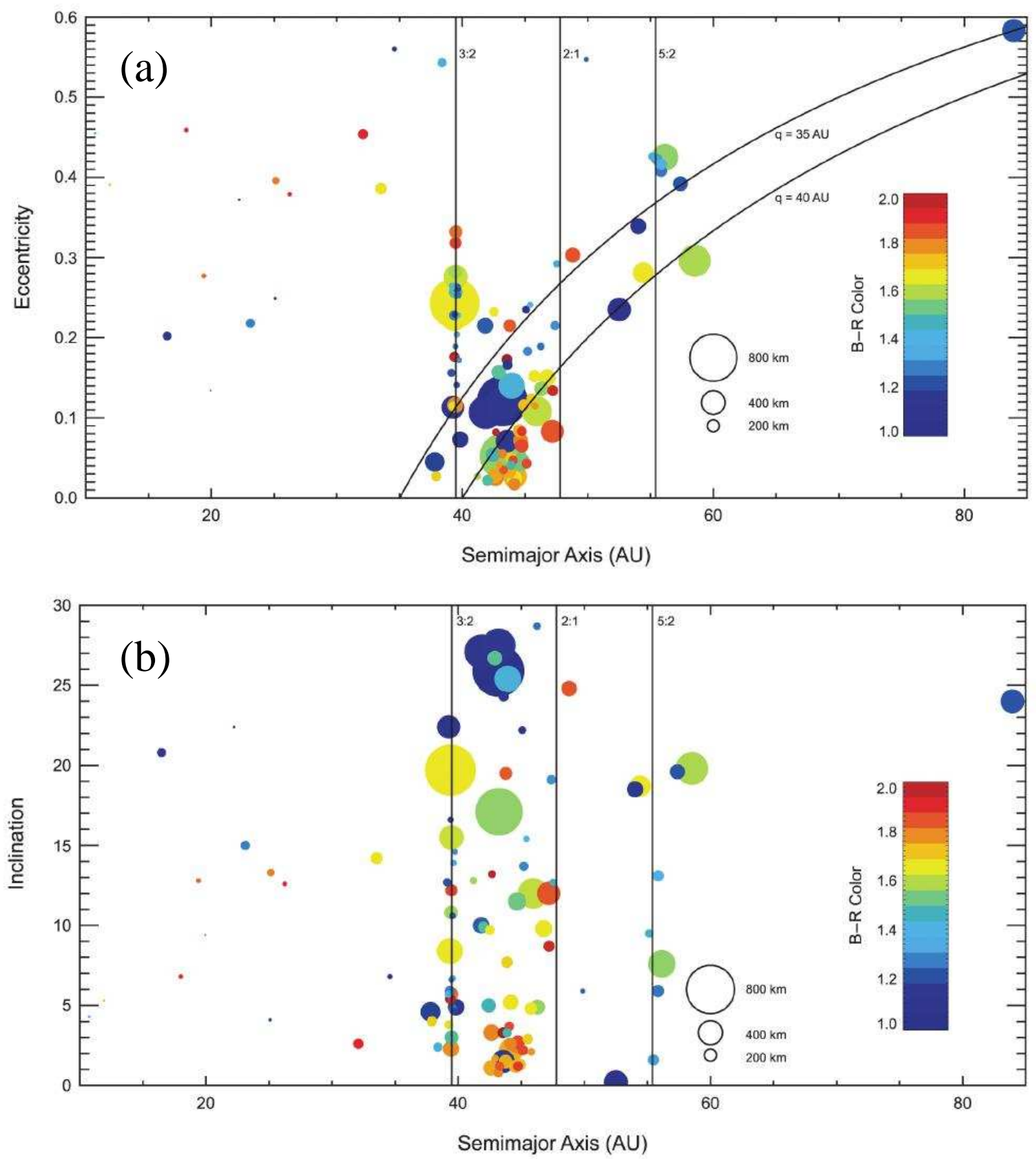

Figura 3.12. Distribución de colores para Centauros y objetos de la Región Transneptuniana, en el espacio de elementos orbitales. Los objetos son graficados a través de círculos proporcionales a sus tamaños, y sus colores son escaleados desde azul a rojo, de acuerdo al valor de sus índices de color $B-R$. Figura extraída de Doressoundiram et al. (2005). 
Haciendo uso de una muestra de 51 Centauros y objetos transneptunianos con índices de color $B-V, V-R, V-I$ y $V-J$, estos autores definieron los siguientes cuatro grupos:

- BB: está representado por objetos que tienen colores neutros respecto al Sol. Sus propiedades espectrales ponen en evidencia la existencia de grandes cantidades de carbono amorfo sobre sus superficies. Por otra parte, las bandas de absorción de $\mathrm{H}_{2} \mathrm{O}$ resultan ser más fuertes que en los otros grupos.

- $R R$ : contiene a los objetos más enrojecidos del Sistema Solar. Los análisis espectrales indican la existencia de hielo de $\mathrm{H}_{2} \mathrm{O}$ sobre sus superficies. Por otra parte, el enrojecimiento significativo manifestado por estos objetos podría implicar la existencia de grandes cantidades de sólidos orgánicos complejos producidos por la irradiación.

- IR: sus miembros resultan ser menos rojos que los representantes del grupo $R R$. Algunos de ellos contienen silicatos hidratados sobre sus superficies.

- $B R$ : es un grupo intermedio entre el $B B$ y el $I R$, aun cuando su color está más relacionado con este último. La mayoría de sus miembros presentan hielo de $\mathrm{H}_{2} \mathrm{O}$ sobre la superficie.

Los análisis indican que a medida que nos movemos desde el grupo neutro $B B$ al enrojecido $R R$, un mayor contenido de material orgánico es requerido para poder ajustar adecuadamente las características espectrales observadas. Los colores típicos asociados a los grupos $B B$ y $B R$ pueden ser alcanzados sin requerir la presencia de material orgánico, o al menos con un escaso porcentaje. Por otra parte, el hielo de $\mathrm{H}_{2} \mathrm{O}$ podría estar presente en todos los espectros de estos grupos. Finalmente, destacamos que los grupos $B B$ y $B R$ tienen colores muy similares a aquellos de los asteroides tipo $C$ y $D$.

\section{Colores}

Las características más importantes concernientes a los colores de Centauros y objetos de la Región Transneptuniana fueron derivadas por Peixinho et al. (2004) y Doressoundiram et al. (2005), entre otros.

La Figura 3.12, extraída de Doressoundiram et al. (2005), representa la distribución de colores para Centauros y objetos de la Región Transneptuniana, en el espacio de elementos orbitales. Los objetos son graficados a través de círculos proporcionales a sus tamaños, y sus colores son escaleados desde azul a rojo, de acuerdo al valor de sus índices de color $B-R$.

A partir de la misma se observan correlaciones entre el color, la inclinación y la distancia perihélica de los Objetos Clásicos. De la Figura 3.12a se aprecia que los objetos con distancias perihélicas $q \leq 40$ UA tienden a ser más azules, mientras que aquellos con distancias perihélicas mayores son más rojos. Además, a partir de la Figura 3.12b, es posible observar que los objetos con alta inclinación orbital tienden a ser más azules que aquellos con inclinaciones menores. Sin embargo, los estudios indican que esta correlación podría estar conectada con aquella que involucra al perihelio. Por otra parte, es importante destacar que las correlaciones mencionadas parecen ser dependientes del tamaño de los objetos. 

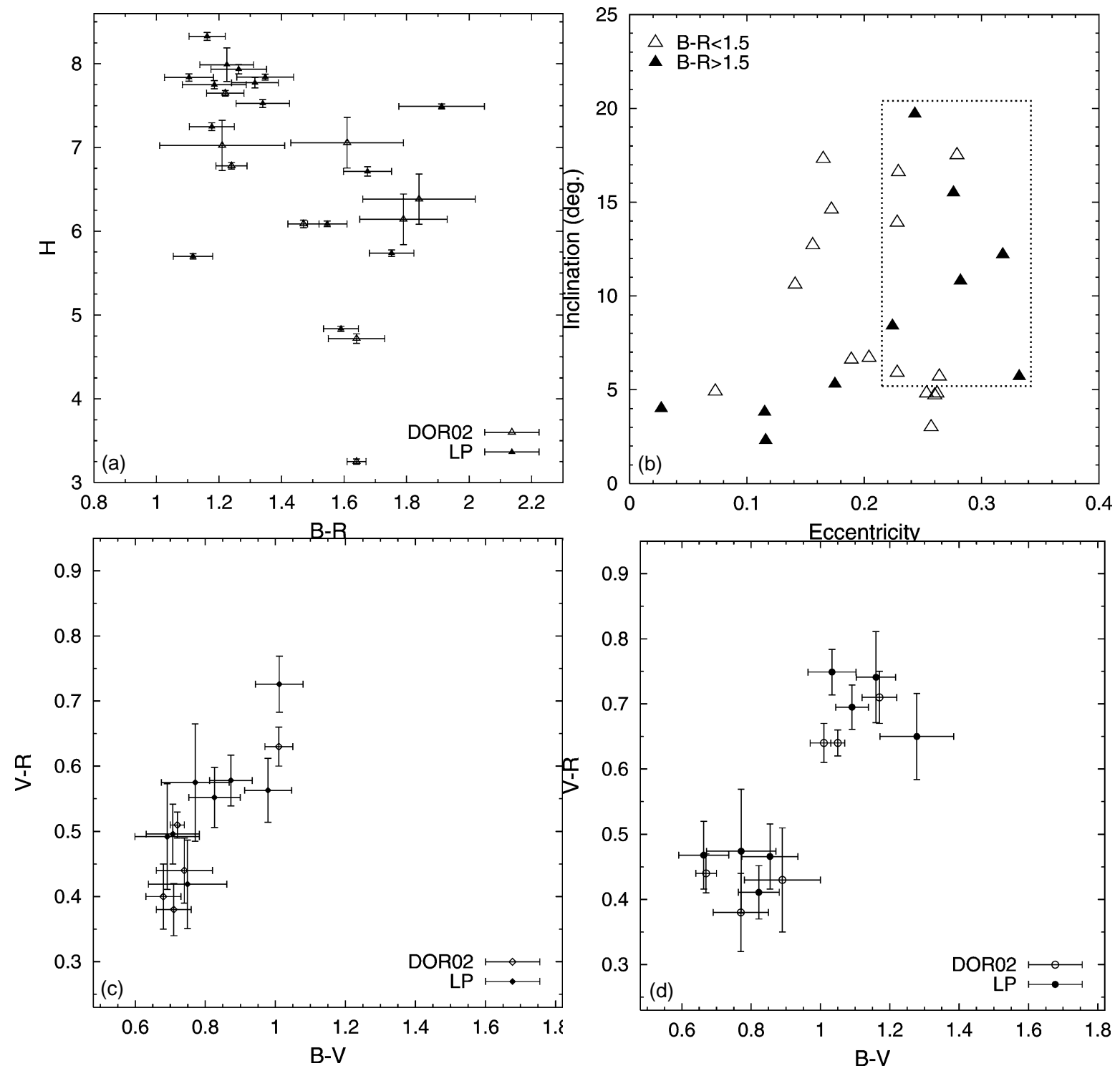

Figura 3.13. a) Distribución de colores $B-R$ de Plutinos, como función de la magnitud absoluta H. Los más pequeños parecen ser más azules, mientras que los más grandes se muestran más rojos. b) Distribución de colores $B-R$ de Plutinos en el plano $(e, i)$. Los diferentes colores se mezclan bien, sin ubicarse en regiones preferenciales. c) Distribución de colores $B-V$ y $V-R$ para los objetos del Disco Dispersado. La escasez de objetos rojos es evidente. d) Distribución de colores $B-V$ y $V-R$ para Centauros. Los colores parecen definir dos diferentes grupos, sugiriendo una bimodalidad. La sigla DOR02 hace referencia a los datos derivados por Doressoundiram et al. (2002) con el Meudon Multicolor Survey, mientras que LP representa el conjunto de datos de Peixinho et al. (2004) obtenidos a partir del ESO Large Program. Figura extraída de Peixinho et al. (2004). 
Si observamos la Figura 3.12b podemos notar que los Objetos Clásicos parecen agruparse en dos poblaciones distintivas: 1) una población de color rojo, representada por un agrupamiento de objetos dinámicamente fríos con inclinaciones $i \lesssim 4.5^{\circ}$, y 2 ) una población con inclinaciones superiores y una mayor dispersión de colores. Este resultado es consistente con aquel propuesto por Brown (2001). Los estudios indican que son necesarias nuevas investigaciones para determinar si las correlaciones mencionadas en el párrafo anterior son debidas a esta subdivisión.

Concerniente a la población de Plutinos, los estudios indican que sus miembros más pequeños resultan ser más azules, mientras que aquellos de mayor tamaño son más rojos (Figura 3.13a). Otro dato de interés es que la distribución de los Plutinos de diferentes colores están bien mezclados en el espacio $(i, e)$ (Figura 3.13b). Este punto es importante, ya que introduce una restricción significativa en los modelos de formación de estas estructuras, tal como se mencionó en la Sección 3.1.4. Por otra parte, la población del Disco Dispersado manifiesta una carencia de objetos rojos (Figura 3.13c).

Un rasgo peculiar observado en estas poblaciones de pequeños cuerpos es la bimodalidad de colores de los Centauros (Figura 3.13d). Esta cualidad no es apreciada en las distribuciones asociadas a los objetos de la Región Transneptuniana.

\section{Densidad}

Hasta la fecha, alrededor de 40 sistemas binarios o múltiples han sido reconocidos en la Región Transneptuniana. Plutón, Haumea y Eris, tres de los más grandes objetos de esta región, forman parte de estas estructuras. Diferentes trabajos han sido desarrollados con el fin de calcular las densidades asociadas a estos grandes objetos de la Región Transneptuniana, haciendo uso de las propiedades orbitales de sus satélites. Buie et al. (2006) analizaron el sistema constituido por Plutón y Caronte, derivando valores para la densidad de estos objetos de $\rho=2 \pm 0.06$ y $\rho=1,65 \pm 0.06 \mathrm{~g} \mathrm{~cm}^{-3}$, respectivamente. De modo similar, Rabinowitz et al. (2006) derivaron una densidad $\rho=3 \pm 0.4 \mathrm{~g} \mathrm{~cm}^{-3}$ para Haumea, mientras que Brown (2006) obtuvo un valor de $\rho=2,26 \pm 0.25 \mathrm{~g} \mathrm{~cm}^{-3}$ para Eris. Las altas densidades asociadas a estos grandes objetos transneptunianos indican que sus interiores deben albergar una cantidad significativa de material rocoso y/o carbonáceo, a diferencia de sus superficies completamente dominadas por hielo.

Recientemente, datos derivados a través del Telescopio Espacial Spitzer permitieron derivar las densidades asociadas a cuatro sistemas binarios pequeños de la Región Transneptuniana. En efecto, los valores obtenidos para (26308) $1998 \mathrm{SM}_{165}$, (47171) $1999 \mathrm{TC}_{36}$, (65489) Ceto/Phorcys y (42355) Tychon/Echidna fueron de $\rho=0,7 \pm_{0,21}^{0,32} \mathrm{~g} \mathrm{~cm}^{-3}$ (Spencer et al. 2006), $\rho=0,5 \pm_{0,2}^{0,3} \mathrm{~g} \mathrm{~cm}^{-3}$ (Stansberry et al. 2006), $\rho=1,38 \pm_{0,32}^{0,65} \mathrm{~g} \mathrm{~cm}^{-3}$ (Grundy et al. 2007) y $\rho=0,47 \pm_{0,10}^{0,18} \mathrm{~g} \mathrm{~cm}^{-3}$ (Grundy et al. 2008), respectivamente. Por otra parte, Takahashi \& Ip (2004) estimaron una densidad $\rho \leq 0.7 \mathrm{~g} \mathrm{~cm}^{-3}$ para $2001 \mathrm{QG}_{298}$. Las bajas densidades de cuatro de estos cinco objetos requieren que los mismos no posean material rocoso en su interior, o que al menos, exista en muy escasas cantidades. Incluso para composiciones de hielo $\mathrm{H}_{2} \mathrm{O}$ puro, las densidades encontradas sugieren diversos espacios vacíos en su estructura interna. Por otra parte, la densidad de Ceto/Phorcys, algo más elevada que el resto, es consistente con una mezcla de hielo y roca. Estos resultados, junto con las conclusiones derivadas por McKinnon et al. (2008), establecen que las densidades de los objetos de la Región 
Transneptuniana se ubican en una amplio rango, desde aproximadamente 0.5 hasta $3 \mathrm{~g} \mathrm{~cm}^{-3}$.

\subsection{Colisiones entre Asteroides - Leyes de Impacto}

El primer intento de analizar la evolución colisional de los asteroides del Cinturón Principal fue desarrollado por Dohnanyi (1969) hace casi cuarenta años. Dohnanyi (1969) modeló analíticamente la evolución colisional de una población de objetos asumiendo a los mismos como esferas de igual densidad con la misma resistencia a impactos por unidad de masa. Dicho modelo incluyó tanto colisiones catastróficas como eventos de craterización, aunque concluyó que el efecto de las craterizaciones resulta ser despreciable. El resultado más importante obtenido a partir del análisis de Dohnanyi (1969) establece que la distribución de tamaños diferencial $^{4}$ de una población en estado estacionario tiene un exponente igual a 3.5. Tiempo después, los trabajos de Greenberg \& Nolan (1989), Williams \& Wetherill (1994) y Tanaka et al. (1996) permitieron confirmar tales resultados.

Sin embargo, la distribución de tamaños de los asteroides del Cinturón Principal difiere significativamente de una ley de potencias con una única pendiente. En efecto, la distribución de tamaños observada del Cinturón Principal presenta una estructura de onda cuyo valor medio está dado por una ley de potencias con un exponente $q$ menor al valor de 3.5 encontrado por Dohnanyi (1969). Esta inconsistencia entre la teoría y las observaciones se debe fundamentalmente a que, contrariamente a las suposiciones de Dohnanyi (1969), la resistencia a impactos de los asteroides es una función del tamaño. En efecto, diversos modelos numéricos (Love \& Ahrens 1996; Melosh \& Ryan 1997; Benz \& Asphaug 1999), argumentos analíticos (Davis et al. 1985; Farinella et al. 1982; Housen \& Holsapple 1990) y estudios de laboratorio (Housen \& Holsapple 1999) han puesto en evidencia dicha dependencia. Para cuerpos pequeños, con diámetros $\lesssim 1 \mathrm{~km}$, las propiedades del material controlan la resistencia a impactos la cual decrece con un aumento de tamaño. Por otra parte, para cuerpos más grandes, la gravedad domina la resistencia a impactos la cual se incrementa con un aumento de tamaño. Como consecuencia de esto, diremos que los asteroides con diámetros $\lesssim 1 \mathrm{~km}$ están en el régimen de cohesión mientras que aquellos de mayor tamaño pertenecen al régimen gravitatorio.

En los diferentes modelos de evolución colisional encontrados en la bibliografía, dos definiciones alternativas son generalmente utilizadas para describir la resistencia a impactos de los asteroides. Ellas son las energías específicas de impacto de fragmentación y dispersión, designadas por $Q_{\mathrm{S}}$ y $Q_{\mathrm{D}}$, respectivamente. $Q_{\mathrm{S}}$ es la cantidad de energía por unidad de masa del blanco necesaria para fragmentar catastróficamente un cuerpo, de modo tal que el fragmento resultante más grande conserve la mitad de la masa del cuerpo original, sin tener en cuenta la reacumulación de los fragmentos. Por otra parte, $Q_{\mathrm{D}}$ representa la cantidad de energía por unidad de masa necesaria para fragmentar un cuerpo y dispersar la mitad de su masa. Para cuerpos pequeños pertenecientes al régimen de cohesión, la energía de ligadura gravitacional es despreciable por lo cual $Q_{\mathrm{S}}$ y $Q_{\mathrm{D}}$ tienen el mismo valor. Para objetos más grandes pertenecientes al régimen gravitatorio, $Q_{\mathrm{D}}$ debe ser mayor que $Q_{\mathrm{S}}$ ya que la gravedad es importante y puede impedir la dispersión de los fragmentos. Las Figuras 3.14a y b muestran las estimaciones de $Q_{\mathrm{S}}$ y $Q_{\mathrm{D}}$ derivadas por diferentes autores, respectivamente.

\footnotetext{
${ }^{4}$ La distribución de tamaños diferencial de una población está dada por $d N=C D^{-p} d D$, donde $D$ es el diámetro, $d N$ es el número de cuerpos en el rango de tamaños $[D, D+d D], C$ es una constante y $p$ es un exponente dado.
} 


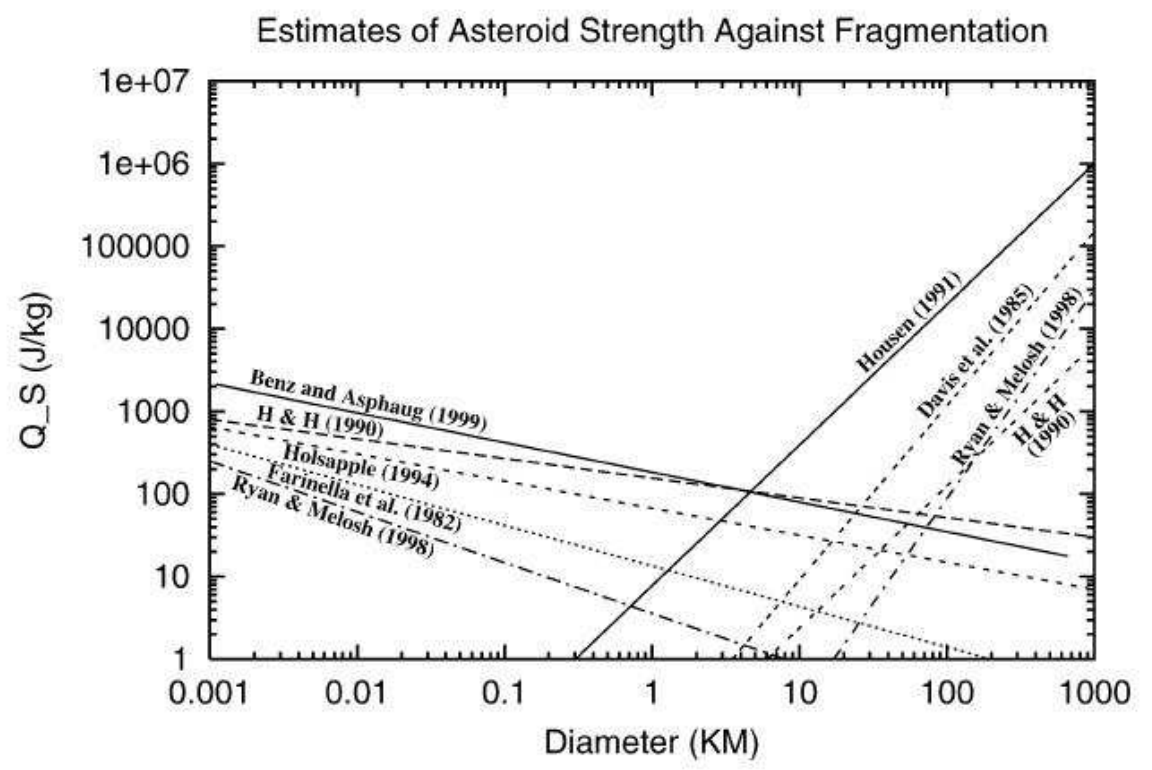

(a)

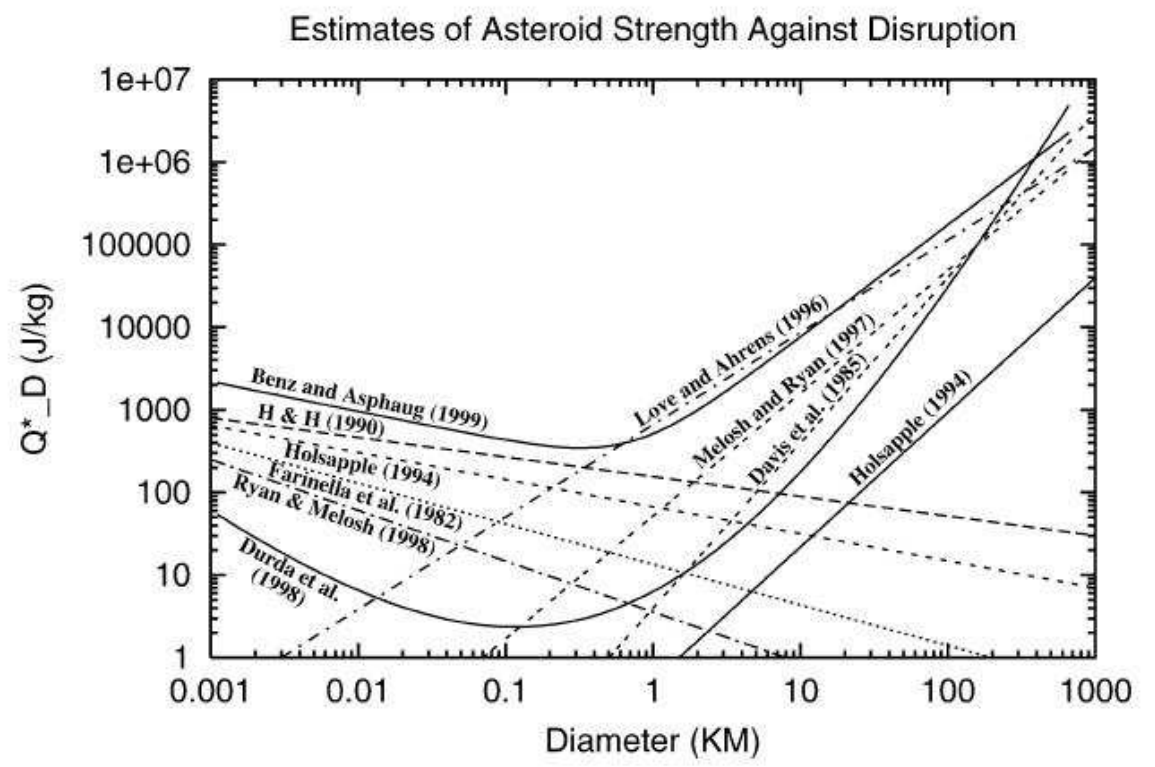

(b)

Figura 3.14. Leyes de Impacto $Q_{\mathrm{S}}$ y $Q_{\mathrm{D}}$ obtenidas por diferentes autores. 
Muchos modelos numéricos de evolución colisional, tales como aquellos desarrollados por Durda (1993), Davis et al. (1994), Durda \& Dermott (1997) y Durda et al. (1998) han analizado los efectos de la dependencia con el tamaño de las leyes de impacto de los asteroides. Dichos trabajos han mostrado que la distribución de tamaños diferencial de una población tiene un exponente mayor a 3.5 cuando la resistencia a impactos decrece con un aumento de tamaño, mientras que tal exponente resulta menor a 3.5 cuando la resistencia a impactos se incrementa. Precisamente este comportamiento da lugar a una estructura de onda que se propaga hacia tamaños mayores que aquel que determina la transición entre el régimen de cohesión y el régimen gravitatorio.

Para analizar estas cuestiones un poco más en detalle, nos remitimos al trabajo desarrollado por O'Brien \& Greenberg (2003), quienes estudiaron los efectos de una resistencia a impactos dependiente del tamaño a partir de un modelo analítico. Estos autores mostraron que si la energía específica de impacto de dispersión $Q_{\mathrm{D}}$ está dada por una ley de potencias del diámetro $D$ de la forma

$$
Q_{\mathrm{D}}=Q_{0} D^{s}
$$

entonces, el exponente de la distribución de tamaños diferencial de una población colisionalmente evolucionada en estado estacionario está dado por

$$
p=\frac{7+\frac{s}{3}}{2+\frac{s}{3}}
$$

Para $s=0$, lo cual corresponde a una resistencia a impactos independiente del tamaño, $p=$ 3.5, reproduciendo la solución clásica de Dohnanyi (1969). Sin embargo, la Ecuación 3.18 nos muestra que si $Q_{\mathrm{D}}$ varía con el tamaño (esto es, si $s \neq 0$ ), $p$ puede diferir significativamente del resultado de Dohnanyi (1969). Para objetos en el régimen de cohesión, la resistencia a impactos decrece con un aumento de tamaño, por lo que $s<0$ y por ende $p<3.5$. Contrariamente, para objetos en el régimen gravitatorio, la resistencia a impactos se incrementa con un aumento de tamaño, por lo que $s>0$ y por ende $p>3.5$.

Para entender cómo este resultado da lugar a una estructura de onda en la distribución de tamaños, consideremos una energía específica de impacto de dispersión $Q_{\mathrm{D}}$ como aquella mostrada en la Figura 3.15a. $Q_{\mathrm{D}}$ consiste de dos leyes de potencia que describen la respuesta colisional de los objetos en el régimen de cohesión y en el régimen gravitatorio y se intersectan en el diámetro de transición $D_{t}$. De acuerdo a lo discutido en el párrafo anterior, la Figura 3.15b nos muestra que la distribución de tamaños diferencial de una población en estado estacionario es más empinada para objetos en el régimen de cohesión que para objetos en el régimen gravitatorio. Llamemos $D_{\mathrm{t}_{\mathrm{dis}}}$ al diámetro del objeto más pequeño capaz de fragmentar a un cuerpo de diámetro $D_{\mathrm{t}}$. Debido a la transición entre el régimen gravitatorio y el régimen de cohesión por debajo del diámetro $D_{\mathrm{t}}$, el número de impactores con un diámetro $D_{t_{\text {dis }}}$ es mayor que aquel que se tendría si se asumiese que todos los cuerpos pertenecen al régimen gravitatorio, llevando a una configuración que no está en estado estacionario. A partir de la Figura 3.15c se observa que debido a este exceso de impactores, el número de cuerpos de diámetro $D_{\mathrm{t}}$ decrece. Finalmente, la Figura $3.15 \mathrm{~d}$ nos muestra que este decrecimiento lleva a un exceso de objetos que pueden ser destruidos por impactores de diámetro $D_{\mathrm{t}}$, lo cual conduce a una disminución en el número de cuerpos mayores y así siguiendo. De este modo, una estructura de onda se forma en la porción de la población asociada a los objetos de mayor tamaño. 

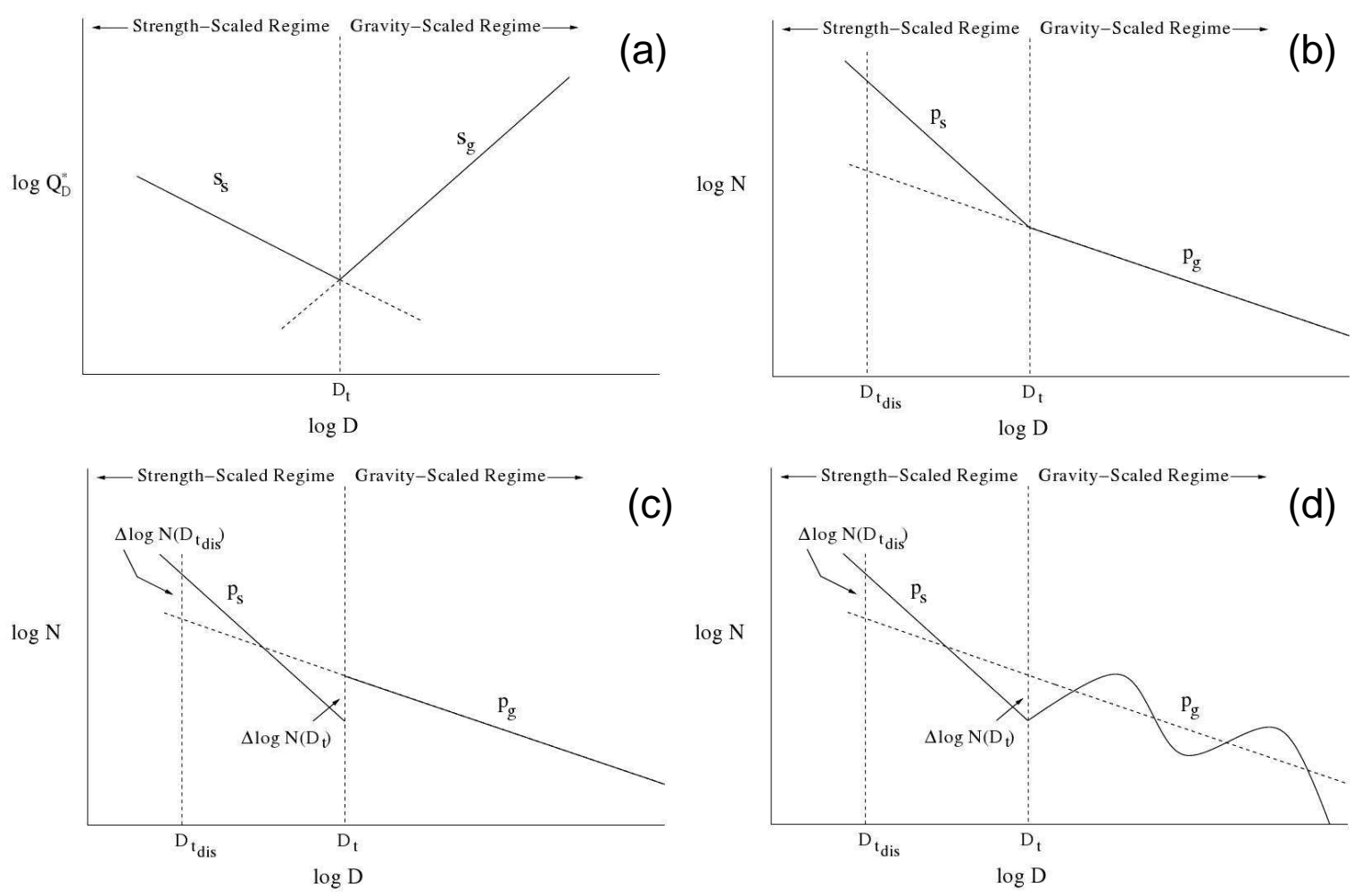

Figura 3.15. Formación de ondas en una población de objetos que evolucionan colisionalmente. La transición entre un régimen de cohesión y un régimen gravitatorio da lugar a la formación de estas estructuras.

Otro de los factores que juega un rol fundamental en la descripción de la resistencia a impactos de un asteroide es el denominado parámetro de inelasticidad $f_{\text {ke }}$, el cual determina que fracción de la energía recibida por un cuerpo es transformada en energía cinética de los fragmentos. Un valor pequeño de $f_{\text {ke }}$ implica que la dispersión de los fragmentos será dificultosa, lo cual se corresponde con un valor grande de $Q_{\mathrm{D}}$. Por el contrario, un valor elevado de $f_{\text {ke }}$ lleva a una dispersión más efectiva, lo cual está de acuerdo con un valor pequeño de $Q_{\mathrm{D}}$ (Davis et al. 1989). El parámetro de ineslaticidad $f_{\mathrm{ke}}$ es pobremente conocido en procesos colisionales. Sin embargo, es aceptado que $f_{\text {ke }}$ puede variar con el tamaño, con la velocidad de impacto y probablemente con las propiedades del material. De acuerdo a lo estudiado por Davis et al. (1989), para cuerpos con diámetros de $\sim 100 \mathrm{~km}, f_{\text {ke }}$ puede ser mayor a 0.1 , mientras que para objetos pequeños, analizados en experimentos de laboratorio, $f_{\text {ke }}$ puede ser menor a 0.01 .

Algunos autores, tales como Durda et al. (1998), usaron a $Q_{\mathrm{D}}$ en lugar de $Q_{\mathrm{S}}$ como parámetro principal en sus modelos de evolución colisional. Sin embargo, O'Brien \& Greenberg (2005) mostraron que la forma general de la población de asteroides evolucionada final está determinada principalmente por $Q_{\mathrm{D}}$, aunque variaciones en $Q_{\mathrm{S}}$ y $f_{\mathrm{ke}}$ pueden afectar tal población final aun cuando $Q_{\mathrm{D}}$ sea siempre la misma. Esta conclusión resulta ser relevante y nos permite justificar la elección de $Q_{\mathrm{S}}$ y $f_{\mathrm{ke}}$ como parámetros de entrada de nuestro modelo colisional, el cual es descripto en detalle en el capítulo siguiente. 


\section{Capítulo 4}

\section{Modelo Colisional}

En este capítulo, presentamos las principales características de nuestro algoritmo destinado a describir el resultado de una colisión entre dos cuerpos. El modelo propuesto está basado en el método desarrollado por Petit \& Farinella (1993), con las correcciones realizadas por O’Brien \& Greenberg (2005) en la determinación de la velocidad de escape.

\subsection{Definiciones}

Para cualquier estudio de evolución colisional, las siguientes cantidades resultan ser fundamentales:

- las energías específicas de impacto de fragmentación y dispersión $Q_{\mathrm{S}}$ y $Q_{\mathrm{D}}$, respectivamente,

- el parámetro de ineslaticidad $f_{\text {ke }}$, el cual determina que fracción de la energía recibida por un cuerpo es transformada en energía cinética de los fragmentos,

- la velocidad media de impacto $V, \mathrm{y}$

- la probabilidad intrínseca de colisión $\left\langle P_{\mathrm{i}}\right\rangle$, la cual determina la probabilidad de colisión por unidad de tiempo y unidad de sección eficaz, para un dado par blanco-proyectil.

El algoritmo numérico que nosotros proponemos permite estudiar el resultado de una colisión entre dos cuerpos en dos regímenes de naturaleza diferente: (1) un régimen catastrófico, donde el fragmento resultante más grande conserva menos del $50 \%$ de la masa del cuerpo original, y (2) un régimen de craterización, donde, como la denominación lo indica, tiene lugar la formación de cráter. La velocidad de impacto $V$ y la energía específica de fragmentación $Q_{\mathrm{S}}$ nos permiten determinar, para un cuerpo dado, si la colisión debe ser estudiada en el régimen catastrófico o en el régimen de craterización. En efecto, la energía cinética relativa en una colisión entre dos cuerpos de masas $M_{1}$ y $M_{2}$ está dada por

$$
E_{\mathrm{rel}}=\frac{1}{2} \frac{M_{1} M_{2}}{M_{1}+M_{2}} V_{\mathrm{rel}}^{2}
$$


donde $V_{\text {rel }}$ es la velocidad de impacto relativa. De acuerdo a las definiciones dadas y asumiendo que esta energía es equiparticionada entre los dos cuerpos colisionantes (Hartmann 1988), para el cuerpo $i$ la fragmentación catastrófica tiene lugar si $E_{\mathrm{rel}}>2 Q_{\mathrm{S}, i} M_{i}$ (Greenberg et al. 1978; Petit \& Farinella 1993), mientras que por debajo de este límite, ocurre un evento de craterización. De este modo, si dos objetos colisionan, la última relación permite determinar si ambos son catastróficamente fragmentados, si uno es craterizado y el otro fragmentado catastróficamente, o ambos son craterizados (lo cual resulta ser muy poco probable teniendo en cuenta los valores actuales asociados a la velocidad de impacto entre asteroides, la cual es de $\sim 5 \mathrm{~km} \mathrm{~s}^{-1}$ en el Cinturón Principal, según lo analizado por Bottke et al. 1994). Para cualquiera de estas posibles salidas, nuestro algoritmo computa la distribución de los fragmentos resultantes desde cada uno de los dos cuerpos colisionantes. En efecto, las Secciones 4.2 y 4.3 describen el tratamiento de una colisión en el régimen catastrófico y en el régimen de craterización, respectivamente. Además, para cualquier de las tres posibles salidas mencionadas, la Sección 4.4 describe los procesos de escape y reacumulación de los fragmentos resultantes, llevando a cabo una determinación de la velocidad de escape.

\subsection{Impactos Catastróficos}

Con el objetivo de modelar la distribución de los fragmentos que resultan a partir de un evento de fragmentación catastrófica, desarrollamos un modelo basado en el algoritmo de Petit \& Farinella (1993).

Si un cuerpo de masa $M_{i}$ es fragmentado catastróficamente, la masa del fragmento resultante más grande será $M_{\max , i}=M_{i} f_{1, i}$, donde $f_{1, i}$ es

$$
f_{\mathrm{l}, i}=\frac{1}{2}\left(\frac{Q_{\mathrm{S}, i} M_{i}}{E_{\mathrm{rel}} / 2}\right)^{1,24}
$$

de acuerdo a resultados experimentales obtenidos por Fujiwara et al. (1977).

Definimos $N_{i}(\geq m)$ como el número de fragmentos del cuerpo $i$ con una masa mayor que m. $N_{i}(\geq m)$ tiene una discontinuidad en $m=M_{\max , i}$ ya que existe sólo un fragmento de masa $M_{\max , i}$ que resulta de la fragmentación catastrófica del cuerpo $i$. Entonces, si $\Theta(x)$ es la Función Escalón de Heaviside (esto es, $\Theta(x)=0$ para $x<0$ y $\Theta(x)=1$ para $x \geq 0$ ), $N_{i}(\geq m)$ puede escribirse como

$$
N_{i}(\geq m)=B_{i} m^{-b_{i}} \Theta\left(M_{\max , i}-m\right)
$$

donde $b_{i}$ es un exponente característico. Además, como $N_{i}\left(\geq M_{\max , i}\right)=1$, entonces a partir de la última ecuación encontramos que $B_{i}=\left(M_{\max , i}\right)^{b_{i}}$. Con el objetivo de calcular el exponente característico $b_{i}$, derivamos la distribución acumulada de masa $M_{i}(\leq m)$, la cual representa la masa total de fragmentos del cuerpo $i$ con una masa menor que $m$. En efecto, $M_{i}(\leq m)$ puede ser calculada por la expresión

$$
M_{i}(\leq m)=\int_{0}^{m} m n_{i}(m) d m
$$


donde $n_{i}(m) d m$ define la distribución diferencial de tamaños de los fragmentos. De acuerdo a la Ecuación $4.3, n_{i}(m) d m$ está dada por

$$
\begin{aligned}
n_{i}(m) d m= & \left\{b_{i} B_{i} m^{-b_{i}-1} \Theta\left(M_{\max , i}-m\right)\right. \\
& \left.+B_{i} m^{-b_{i}} \delta\left(m-M_{\max , i}\right)\right\} d m,
\end{aligned}
$$

donde $\delta(x)=d \Theta(x) / d x$ es la Función Delta de Dirac. Luego, insertando esto en la Ecuación 4.4 y usando que $B_{i}=\left(M_{\max , i}\right)^{b_{i}}$, entonces $M_{i}(\leq m)$ será escrita como

$$
\begin{aligned}
M_{i}(\leq m)= & \frac{b_{i} M_{\max , i}^{b_{i}}}{1-b_{i}} m^{1-b_{i}}\left\{1-\Theta\left(m-M_{\max , i}\right)\right\} \\
& +\frac{M_{\max , i}}{1-b_{i}} \Theta\left(m-M_{\max , i}\right) .
\end{aligned}
$$

A partir de esta expresión, es posible derivar una relación entre $f_{1, i}$, dada por la Ecuación 4.2, y el exponente característico $b_{i}$. En efecto, la ley de conservación de la masa implica que $M_{i}\left(\leq M_{\max , i}\right)=M_{i}$; luego, a partir de la Ecuación 4.6, derivamos la condición

$$
M_{i}=\frac{M_{\max , i}}{1-b_{i}}
$$

y, ya que $M_{\max , i}=M_{i} f_{1, i}$, entonces

$$
b_{i}=1-f_{1, i} .
$$

De este modo, si $f_{1, i}$ es calculada por la Ecuación $4.2, b_{i}$ puede ser derivada a parir de la expresión 4.8. Con esto, cada parámetro presente en la Ecuación 4.3 queda determinado y luego, dicha expresión puede ser usada para calcular la distribución de los fragmentos que resultan a partir de un evento de fragmentación catastrófica.

\subsection{Impactos Craterizantes}

Por debajo del límite de fragmentación catastrófico $\left(E_{\mathrm{rel}}<2 Q_{\mathrm{S}, i} M_{i}\right)$, la colisión da lugar a la formación de un cráter sobre el cuerpo $i$. Nuevamente, usamos el algoritmo de Petit \& Farinella (1993) con el objetivo de calcular la distribución de fragmentos resultantes de un evento de craterización. La masa $M_{\text {crat }, i}$ excavada desde el cráter formado puede ser calculada a partir de las siguientes relaciones

$$
M_{\mathrm{crat}, i}= \begin{cases}\alpha E_{\mathrm{rel}} & \text { si } E_{\mathrm{rel}} \leq \beta \\ \frac{9 \alpha}{200 Q_{\mathrm{S}, i} \alpha-1} E_{\mathrm{rel}}+\frac{M_{i}}{10} \frac{1-20 Q_{\mathrm{S}, i} \alpha}{1-200 Q_{\mathrm{S}, i} \alpha} & \text { si } E_{\mathrm{rel}}>\beta\end{cases}
$$

donde $\beta=M_{i} / 100 \alpha$. El parámetro $\alpha$, conocido como coeficiente de excavación, depende de las propiedades del material y su valor se encuentra en el rango que va desde $4 \times 10^{-4}$ a $10^{-5}$ $\mathrm{s}^{2} \mathrm{~m}^{-2}$ para materiales blandos y duros, respectivamente (Stoeffler et al. 1975; Dobrovolskis \& Burns 1984). Como la Ecuación 4.9 indica, el modelo propuesto por Petit \& Farinella (1993) asume una dependencia lineal de $M_{\mathrm{crat}, i}$ con $E_{\mathrm{rel}}$ en una forma tal que para cráteres con una masa excavada menor al $1 \%$ de la masa del blanco, $M_{\text {crat }, i}$ es proporcional a $E_{\text {rel }}$, mientras que para cráteres mayores, los coeficientes de la relación lineal son seleccionados de modo tal 
que la masa excavada del cráter más grande posible sea del $10 \%$ de la masa del blanco, lo cual está de acuerdo con los estudios desarrollados por Nolan et al. (1996).

En esta clase de impactos, el cuerpo craterizado sobreviviente tiene una masa $M_{i}-M_{\text {crat }, i}$. $\mathrm{Al}$ igual que en el caso de un evento de fragmentación catastrófico, nosotros también modelamos la distribución de tamaño de los fragmentos resultantes de un impacto craterizante a partir de una ley de potencia con un única pendiente. Es importante tener en cuenta que las expresiones derivadas para tratar un impacto catastrófico pueden ser usadas para estudiar un impacto craterizante reemplazando la masa del blanco $M_{i}$ por $M_{\text {crat }, i}$. De este modo, la masa del fragmento más grande eyectado desde el cráter será $f_{1, i} M_{\text {crat }, i}$, donde $f_{1, i}=0,2$ ya que, de acuerdo con Melosh (1989), $b_{i}=0,8$ para cualquier evento craterizante.

\subsection{Escape y Reacumulación de Fragmentos}

Una vez que calculamos la distribución de los fragmentos asociados con cada uno de los cuerpos que participan en una colisión, es necesario determinar el destino final de los mismos. Un fragmento con una velocidad $V$ mayor a la velocidad de escape $V_{\text {esc }}$ del sistema que constituyen los dos cuerpos colisionantes terminará escapando. Por el contrario, aquellos fragmentos con velocidades menores a $V_{\text {esc }}$ serán reacumulados sobre el remanente más grande.

De acuerdo a lo sugerido por Campo Bagatin et al. (1994b), es posible adoptar dos modelos diferentes para estudiar el escape y la reacumulación de los fragmentos generados en una colisión:

1. Un "Modelo Acumulativo", en el cual no hay relación entre la masa y la velocidad de los fragmentos. Este modelo sólo asume que la fracción de la masa de los fragmentos eyectados desde el cuerpo $i$ con velocidades mayores que un dado valor $V$ está dada por

$$
f(\geq V)=\frac{M_{i}(\geq V)}{M_{i}}=\left(\frac{V}{V_{\min }}\right)^{-k}
$$

donde $V_{\min }$ es un límite inferior para la velocidad de los fragmentos. Gault et al. (1963) derivaron una relación tal, con una valor de $k$ de $\sim 9 / 4$.

2. Un "Modelo Masa-Velocidad", tal como aquel propuesto por Petit \& Farinella (1993). Este modelo asume una correlación entre la velocidad de eyección y la masa de los fragmentos, en acuerdo con resultados experimentales derivados por Nakamura \& Fujiwara 1991, Nakamura et al 1992, Giblin et al. 1994 y Giblin 1998. Esta relación masavelocidad se expresa en la forma siguiente

$$
V=C_{i} m^{-r_{i}}
$$

donde $C_{i}$ es un coeficiente constante y $r_{i}$ es un dado exponente.

Es posible encontrar una relación entre el exponente $k$ del modelo acumulativo y el exponente $r_{i}$ del modelo masa-velocidad. En efecto, a partir de la Ecuación 4.11, escribimos $m(V)$ como

$$
m(V)=\left(\frac{V}{C_{i}}\right)^{-1 / r_{i}} .
$$


Insertando esta expresión en la distribución acumulada de masa dada por la Ecuación 4.6 y considerando masas menores que $M_{\max , i}$, tenemos que

$$
\begin{aligned}
M_{i}(\geq V) & =\frac{b_{i} M_{\max , i}^{b_{i}}}{1-b_{i}} m(V)^{1-b_{i}} \\
& =\frac{b_{i} M_{\max , i}^{b_{i}}}{1-b_{i}}\left(\frac{V}{C_{i}}\right)^{\left(1-b_{i}\right) / r_{i}} .
\end{aligned}
$$

Las Ecuaciones 4.10 y 4.13 nos permiten ver que

$$
r_{i}=\frac{1-b_{i}}{k}
$$

donde $b_{i}$ se obtiene a partir de la Ecuación 4.8 para un evento catastrófico y es igual a 0.8 para un impacto craterizante.

En nuestro estudio, seguimos el modelo propuesto por Petit \& Farinella (1993) para calcular la distribución de velocidades de los fragmentos. En efecto, el Modelo Masa-Velocidad adopta la siguiente expresión

$$
\begin{array}{rll}
V=C_{i} m^{-r_{i}} & \text { para } & \bar{M}_{i} \leq m \leq M_{\max , i} \\
V=V_{\max } & \text { para } & m<\bar{M}_{i},
\end{array}
$$

donde, imponiendo continuidad, $\bar{M}_{i}=\left(V_{\max } / C_{i}\right)^{-1 / r_{i}}$. Asumimos que $V_{\max }$ es el valor máximo adoptado para la velocidad de los fragmentos. La inclusión de este límite superior para las velocidades de eyección está motivada por una razón física que establece que un fragmento nunca puede ser eyectado con una velocidad superior a la velocidad del sonido en el material, la cual asumimos con un valor de $3000 \mathrm{~m} \mathrm{~s}^{-1}$ (O'Brien \& Greenberg 2005). Si bien este valor parecería ser demasiado elevado (Vokrouhlický et al. 2006), discutiremos en la Sección 5.7.5 la dependencia de nuestras simulaciones sobre este parámetro de entrada. Por otra parte, el coeficiente constante $C_{i}$ puede ser calculado a partir de una ecuación de conservación de la energía. Asumiendo que la energía cinética relativa $E_{\text {rel }}$ de la colisión es particionada equitativamente entre el blanco y el proyectil (Hartmann 1988), el cuerpo $i$ recibirá una energía $E_{i}=E_{\mathrm{rel}} / 2$ en el impacto. A partir de esto, definimos $E_{\mathrm{fr}, i}=f_{\mathrm{ke}} E_{i}$ como la energía cinética de los fragmentos que resultan de tal cuerpo. Si bien el parámetro de ineslaticidad $f_{\text {ke }}$ es pobremente conocido, de acuerdo a lo propuesto por O'Brien \& Greenberg (2005), el mismo es expresado como

$$
f_{\mathrm{ke}}=f_{\mathrm{ke}_{0}}\left(\frac{D}{1000 \mathrm{~km}}\right)^{\gamma}
$$

donde $\gamma$ es del orden de 0.5 (siempre entre 0 y 1) y $f_{\mathrm{ke}_{0}}$, el valor para objetos de $1000 \mathrm{~km}$ de diámetro, está entre $\sim 0.05-0.3$, lo cual está de acuerdo con las estimaciones de $f_{\text {ke }}$ en grandes impactos (Davis et al. 1989). Por otra parte, si bien $E_{\mathrm{fr}, i}=f_{\mathrm{ke}} E_{i}$, la energía cinética de los fragmentos $E_{\mathrm{fr}, i}$ que resultan del cuerpo $i$ puede ser escrita también siguiendo el modelo masa velocidad propuesto. En efecto

$$
\begin{aligned}
E_{\mathrm{fr}, i}= & \lim _{\epsilon \rightarrow 0} \int_{\bar{M}_{i}}^{M_{\max , i}-\epsilon} \frac{V^{2}}{2} m n_{i}(m) d m+\frac{V_{\max }^{2}}{2} M\left(\leq \bar{M}_{i}\right) \\
& +\lambda_{i} \frac{V_{\mathrm{lf}, i}^{2}}{2} M_{\max , i}
\end{aligned}
$$


donde $n_{i}(m) d m$ está dado por la Ecuación 4.5, y el último término es la energía cinética del fragmento más grande resultante del cuerpo $i$. Los estudios experimentales desarrollados por Fujiwara \& Tsukamoto (1980) y Nakamura \& Fujiwara (1991) indican que el fragmento más grande que resulta de una colisión catastrófica tiene una energía cinética despreciable en el sistema de referencia del centro de masa. Por otra parte, en un evento craterizante, el fragmento más grande de masa $M_{\mathrm{max}, i}=f_{\mathrm{l}, i} M_{\mathrm{crat}, i}\left(\right.$ con $\left.f_{\mathrm{l}, i}=0,2\right)$ tiene una velocidad $V_{\mathrm{lf}, i}$ dada por

$$
V_{\mathrm{lf}, i}=C_{i} M_{\max , i}^{-r_{i}} .
$$

Para tener en cuenta esta diferencia, insertamos el término correspondiente en la ecuación de conservación de la energía multiplicado por un factor $\lambda_{i}$, donde $\lambda_{i}$ será 0 para un evento catastrófico y 1 para un evento craterizante.

La Ecuación 4.17 es una integral de $m$. Ya que el integrando es escrito en términos de $\delta\left(m-M_{\max , i}\right)$ por la Ecuación 4.5, si uno desea resolver esta integral sobre el rango $\left(\bar{M}_{i}, M_{\max , i}\right)$, es necesario introducir $\epsilon$ y evaluar $\operatorname{lím}_{\epsilon \rightarrow 0}$. Una vez que $V$ es escrita en términos de $m$ (Ecuación 4.15), tal integral puede ser evaluada. De este modo

$$
\begin{aligned}
E_{\mathrm{fr}, i}= & \lambda_{i} C_{i}^{2} \frac{M_{\max , i}^{1-2 r_{i}}}{2}+\frac{V_{\max }^{2}}{2} \frac{b_{i}}{1-b_{i}} M_{\max , i}^{b_{i}} \bar{M}_{i}^{1-b_{i}} \\
& +\frac{C_{i}^{2}}{2} \frac{b_{i} M_{\max , i}^{b_{i}}}{1-b_{i}-2 r_{i}}\left[M_{\max , i}^{1-b_{i}-2 r_{i}}-\bar{M}_{i}{ }^{1-b_{i}-2 r_{i}}\right] \\
= & C_{i}^{k_{i}} b_{i} \frac{M_{\max , i}^{b_{i}} V_{\max }^{2-k_{i}}}{2}\left[\frac{1}{1-b_{i}}-\frac{1}{1-b_{i}-2 r_{i}}\right] \\
& +C_{i}^{2} \frac{M_{\max , i}^{1-2 r_{i}}}{2}\left[\lambda_{i}+\frac{b_{i}}{1-b_{i}-2 r_{i}}\right] .
\end{aligned}
$$

A partir de esto, el coeficiente constante $C_{i}$ está dado por la solución de la ecuación

$$
a C_{i}^{k_{i}}+b-C_{i}^{2}=0
$$

donde $a$ y $b$ están dados por

$$
\begin{aligned}
& a=M_{\max , i}^{2 r_{i}+b_{i}-1} V_{\max }^{2-k_{i}}\left[\frac{2 b_{i} r_{i}}{\left[\left(1-2 r_{i}-b_{i}\right) \lambda_{i}+b_{i}\right]\left(1-b_{i}\right)}\right] \\
& b=2 M_{\max , i}^{2 r_{i}-1}\left[\frac{1-b_{i}-2 r_{i}}{\left(1-2 r_{i}-b_{i}\right) \lambda_{i}+b_{i}}\right] E_{\mathrm{fr}, i},
\end{aligned}
$$

y $E_{\mathrm{fr}, i}=f_{\mathrm{ke}} E_{i}$.

Una vez que la distribución de velocidades de los fragmentos ha sido construida para cada uno de los dos cuerpos que participan de una colisión, es necesario calcular la velocidad de escape efectiva $V_{\text {esc }}$ del sistema constituido por los cuerpos colisionantes. Para hacer esto, usamos el método desarrollado por Petit \& Farinella (1993) con las correcciones hechas por O'Brien \& Greenberg (2005). De este modo, calculamos la velocidad de escape $V_{\text {esc }}$ usando una ecuación de balance de energía, la cual puede ser escrita como

$$
\frac{1}{2} M^{*} V_{\mathrm{esc}}^{2}+W_{\mathrm{tot}}=W_{\mathrm{fr}, 1}+W_{\mathrm{fr}, 2}+W_{\mathrm{h}}
$$


donde $M^{*}=M_{1}-M_{\max , 1}+M_{2}-M_{\max , 2}$ si los dos cuerpos colisionantes son catastróficamente fragmentados, $M^{*}=M_{\mathrm{crat}, 1}+M_{2}-M_{\max , 2}$ si el cuerpo 1 es craterizado y el cuerpo 2 es fragmentado catastróficamente, y $M^{*}=M_{\text {crat }, 1}+M_{\text {crat,2 }}$ si ambos cuerpos son craterizados. El término $W_{\text {tot }}$ es la energía potencial gravitacional total de los dos cuerpos colisionantes inmediatamente antes del impacto, y puede ser calculado a partir de la siguiente expresión

$$
W_{\mathrm{tot}}=-\frac{3 G M_{1}^{5 / 3}}{5 Q}-\frac{3 G M_{2}^{5 / 3}}{5 Q}-\frac{G M_{1} M_{2}}{Q M_{1}^{1 / 3}+Q M_{2}^{1 / 3}},
$$

donde el parámetro $Q$ es

$$
Q=\left(\frac{4 \pi \rho}{3}\right)^{-1 / 3}
$$

y $\rho$ es la densidad de los objetos. Por otra parte, el término $W_{\mathrm{fr}, i}$ representa la energía potencial gravitacional de los fragmentos del cuerpo $i$ resultantes de la colisión. Si el cuerpo $i$ es fragmentado catastróficamente, $W_{\mathrm{fr}, i}$ estará dado por

$$
\begin{aligned}
W_{\mathrm{fr}, i} & =-\frac{3}{5} \frac{G}{Q} \int_{m=0}^{m=\infty} m^{5 / 3} n_{i}(m) d m \\
& =-\frac{3 G}{Q} \frac{M_{\mathrm{max}, i}^{5 / 3}}{5-3 b_{i}},
\end{aligned}
$$

mientras que si el cuerpo $i$ es craterizado, $W_{\mathrm{fr}, i}$ adoptará la siguiente expresión

$$
\begin{aligned}
W_{\mathrm{fr}, i} & =-\frac{3}{5} \frac{G}{Q} \int_{m=0}^{m=\infty} m^{5 / 3} n_{2}(m) d m-\frac{3 G\left(M_{i}-M_{\mathrm{crat}, i}\right)^{5 / 3}}{5 Q} \\
& =-\frac{G}{Q} M_{\max , i}^{5 / 3} \frac{3}{5-3 b_{i}}-\frac{3 G\left(M_{i}-M_{\mathrm{crat}, i}\right)^{5 / 3}}{5 Q} .
\end{aligned}
$$

El término $W_{\mathrm{h}}$ es una estimación de la energía potencial gravitacional de los fragmentos cuando los mismos son separados a una distancia del orden del radio de Hill de la masa colisionante total en el campo gravitatorio de la masa central $M_{\mathrm{o}}$ y a una distancia orbital $r_{\mathrm{o}}$. Si ambos cuerpos son fragmentados catastróficamente, $W_{\mathrm{h}}$ estará dado por

$$
W_{\mathrm{h}}=-\frac{3 G\left(M_{1}+M_{2}\right)^{5 / 3}}{5} \frac{\left(3 M_{\mathrm{o}}\right)^{1 / 3}}{R_{\mathrm{o}}},
$$

donde $M_{\mathrm{o}}$ es la masa del Sol y $r_{\mathrm{o}}$ es el radio orbital a la cual ocurre la colisión. Por otra parte, de acuerdo a lo sugerido por O'Brien \& Greenberg (2005), si el cuerpo 1 es craterizado y el cuerpo 2 es fragmentado catastróficamente, el término $W_{\mathrm{h}}$ debe ser escrito como

$$
W_{\mathrm{h}}=-\frac{3 G\left(M_{2}+M_{\mathrm{crat}, 1}\right)\left(M_{1}-M_{\mathrm{crat}, 1}\right)^{2 / 3}}{2} \frac{\left(3 M_{\mathrm{o}}\right)^{1 / 3}}{R_{\mathrm{o}}}
$$

mientras que ambos cuerpos son craterizados, el término $W_{\mathrm{h}}$ tiene la forma

$$
\begin{aligned}
W_{\mathrm{h}}= & -\frac{3 G\left(M_{1}+M_{2}-M_{\text {crat }, 1}-M_{\text {crat }, 2}\right)^{2 / 3}}{2} \\
& \times \frac{\left(M_{\text {crat }, 1}+M_{\text {crat }, 2}\right)}{2} \frac{\left(3 M_{\mathrm{o}}\right)^{1 / 3}}{R_{\mathrm{o}}} .
\end{aligned}
$$




\section{Modelo Colisional}

Una vez que los diferentes términos $W$ son calculados, es posible encontrar la velocidad de escape $V_{\text {esc }}$ a partir de la ecuación de balance de energía correspondiente (Ecuación 4.22). Conociendo $V_{\text {esc }}$, el proceso de reacumulación de los fragmentos puede ser tratado de un modo muy sencillo. En efecto, aquellos fragmentos que sean eyectados con una velocidad $V<V_{\text {esc }}$ serán reacumulados sobre los dos remanentes más grandes producidos individualmente por los cuerpos colisionantes ${ }^{1}$, dando lugar a un único objeto reacumulado. Por otra parte, aquellos fragmentos que sean eyectados con una velocidad $V>V_{\text {esc }}$ escaparán del campo gravitacional de los dos impactores. El destino de estos fragmentos será determinado a partir de los criterios dinámicos que serán discutidos para cada población de pequeños cuerpos particular que sea estudiada.

\footnotetext{
${ }^{1}$ Tengamos presente que si un objeto de masa $M$ recibe un impacto catastrófico, el remanente más grande será el fragmento de masa $M f_{1}$, con $f_{1}$ dado por la Ecuación 4.2. Por otra parte, si un objeto de masa $M$ recibe un impacto craterizante, el remanente más grande será el cuerpo craterizado sobreviviente de masa $M-M_{\text {crat }}$, con $M_{\text {crat }}$ dado por la Ecuación 4.9
} 


\section{Capítulo 5}

\section{Asteroides del Cinturón Principal y NEAs}

\subsection{Introducción}

Los resultados presentados en este capítulo fueron publicados en Astronomy \& Astrophysics, y pueden encontrarse bajo la referencia de Elía \& Brunini (2007a).

Hace más de 50 años, Öpik (1951) y Piotrowski (1953) desarrollaron trabajos pioneros destinados a evaluar la frecuencia de colisiones asteroidales en el Cinturón Principal, revelando la importancia de los procesos colisionales en la evolución de estos pequeños cuerpos. Dada la relevancia de la actividad colisional en estas regiones del Sistema Solar, diversos autores desarrollaron modelos numéricos y analíticos con el objetivo de analizar la evolución colisional de los asteroides. En efecto, Davis et al. (1979) estudiaron numéricamente la evolución colisional de diversas poblaciones de asteroides, analizando sus distribuciones de tamaño, evolución rotacional y velocidades de impacto. Luego, Farinella et al. (1982) estudiaron el rol de las colisiones catastróficas en la evolución de los asteroides. Años después, Davis et al. (1985) desarrollaron estudios numéricos de la evolución colisional de asteroides, teniendo en cuenta restricciones dadas por la distribución de tamaños actual, la preservación de la corteza basáltica de Vesta y el número de las principales familias de Hirayama en el Cinturón Principal. Siguiendo con esta línea de investigación, Davis et al. (1989) discutieron el efecto de las colisiones sobre los tamaños y las tasas de rotación de los asteroides observados. Más tarde, Farinella et al. (1992) analizaron la evolución simultánea de los tamaños y tasas de rotación de los asteroides debido a efectos colisionales sobre la historia del Sistema Solar. Inmediatamente después, Durda (1993) desarrolló un nuevo modelo numérico para estudiar la evolución colisional de asteroides, presentando restricciones sobre la resistencia a impactos. Sobre la misma línea de investigación, Davis et al. (1994) analizaron la evolución colisional de asteroides asumiendo diferentes leyes de fragmentación. Al mismo tiempo, Campo Bagatin et al. (1994a) desarrollaron un modelo de evolución colisional de asteroides con el objetivo de estudiar una relación entre la velocidad y la masa de los fragmentos generados a partir de un evento de impacto. Luego, Durda et al. (1998) analizaron la forma de la distribución de tamaños del Cinturón Principal, haciendo uso de leyes de impacto derivadas a partir de modelos hidrodinámicos. 
La población de asteroides del Cinturón Principal está determinada fundamentalmente por procesos colisionales. Sin embargo, existen diversos mecanismos dinámicos que pueden tener una influencia relevante sobre la evolución de estos pequeños cuerpos. Tal como destacamos en la Sección 3.1.2, las resonancias orbitales entre los asteroides y los planetas así como también el efecto Yarkovsky juegan un rol dominante en remover material desde el Cinturón Principal. Además, estos mecanismos adquieren un interés relevante ya que los mismos llevan a una conexión entre el Cinturón Principal y la población de NEAs. De este modo, un modelo completo destinado a analizar los asteroides del Cinturón Principal y los NEAs debe seguir la evolución de ambas poblaciones de manera simultánea. Además, dicho modelo debe ser capaz de incluir procesos colisionales y dinámicos, ya que sin mecanismos dinámicos, la población de NEAs no podría generarse, y sin evolución colisional, no habría nuevos fragmentos y los cuerpos removidos del Cinturón Principal se perderían sin ser continuamente regenerados.

Recientemente, Bottke et al. (2005a), O’Brien \& Greenberg (2005) y Bottke et al. (2005b) desarrollaron trabajos destinados a analizar la evolución del Cinturón Principal y la población de NEAs. En efecto, Bottke et al. (2005a) desarrollaron un código de evolución colisional con el objetivo de estudiar la pérdida de masa en la región del cinturón de asteroides desde el final de la época de acreción hasta nuestros días. Estos autores encontraron que la distribución de tamaños del Cinturón Principal es predominantemente un fósil producida en los primeros años de evolución colisional, cuando el cinturón de asteroides resultaba ser mucho más masivo. El trabajo presentado por Bottke et al. (2005a) ha permitido analizar algunas cuestiones relacionadas con la forma de la distribución de tamaños inicial del cinturón de asteroides, la estabilidad del Cinturón Principal y la población de NEAs, la historia colisional de (4) Vesta, la frecuencia de disrupción entre asteroides, tasas de rotación y el tamaño estimado del Cinturón Principal primordial. Por otra parte, el estudio desarrollado por O'Brien \& Greenberg (2005) modela la evolución del Cinturón Principal de asteroides, los NEAs y los Objetos Transneptunianos. En particular, estos autores desarrollaron un código numérico para modelar la evolución simultánea del Cinturón Principal y la población de NEAs, considerando procesos colisionales, mecanismos dinámicos asociados a las resonancias orbitales y el efecto Yarkovsky. Este código numérico es capaz de satisfacer las principales restricciones observacionales asociadas con estas poblaciones de pequeños cuerpos, tales como sus distribuciones de tamaños, la historia colisional de (4) Vesta, el número de familias de asteroides y el tiempo de exposición a los rayos cósmicos de los meteoritos. Más tarde, Bottke et al. (2005b) desarrollaron un estudio destinado a analizar la historia colisional del Cinturón Principal de asteroides combinando el algoritmo delineado por Bottke et al. (2005a) y los resultados dinámicos derivados por Petit et al. (2001). Los resultados obtenidos por estos autores son consistentes con datos observacionales asociados a la distribución de tamaños del Cinturón Principal, el número de familias de asteroides producidas a partir de cuerpos precursores mayores a $100 \mathrm{~km}$, la historia colisional de (4) Vesta y el flujo de impactores sobre la Tierra y la Luna durante los últimos $3 \times 10^{9}$ años de evolución. Además, este modelo también permite determinar la población de NEAs, la cual es usada para explorar algunas cuestiones concernientes a los pequeños cráteres formados sobre Mercurio, la Luna y Marte.

Aquí, nosotros desarrollamos un algoritmo numérico de evolución colisional que tiene en cuenta los principales mecanismos dinámicos presentes en el Cinturón Principal de asteroides y la población de NEAs. Entre los trabajos de Bottke et al. (2005a), O'Brien \& Greenberg (2005) y Bottke et al. (2005b), el segundo es el más similar al nuestro, aunque existen algunas diferencias relevantes en las poblaciones del modelo, los parámetros colisionales y el trata- 
miento de la evolución dinámica. En efecto, la diferencia más notable entre aquellos trabajos y nuestro estudio es que nuestro modelo propone dividir el cinturón de asteroides en tres regiones diferenciadas cuyos límites están dados por las resonancias poderosas $\nu_{6}, 3: 1$ y 5:2, lo cual nos ha permitido desarrollar un análisis más riguroso del Cinturón Principal de asteroides y la población de NEAs. El modelo propuesto aquí mejora aquellos presentados por Bottke et al. (2005a), O'Brien \& Greenberg (2005) y Bottke et al. (2005b), siendo capaz de analizar algunas cuestiones relacionadas con la mezcla de material en el cinturón de asteroides, el origen de los NEAs y la inyección colisional dentro de las resonancias poderosas.

\subsection{Poblaciones Iniciales}

El Cinturón Principal de asteroides se extiende aproximadamente desde las 2 hasta las 3.4 UA del Sol. Como ya hemos dicho en la Sección 3.1.2, las resonancias poderosas más importantes localizadas en esta región son la resonancia secular $\nu_{6}$, la cual determina su borde interno, y las resonancias de movimientos medios 3:1, 5:2 y 2:1 con Júpiter a $\sim 2.5,2.82$ y 3.27 UA del Sol, respectivamente. La Figura 5.1 nos muestra un histograma que representa el número de asteroides del Cinturón Principal como función del semieje mayor. Teniendo en cuenta que menos del $2 \%$ de la población del cinturón de asteroides está localizada entre las 3.27 y 3.4 UA, hemos decidido asumir que la resonancia 2:1 determina el límite exterior del Cinturón Principal.

Con el objetivo de estudiar el flujo de objetos dentro de estas resonancias como consecuencia de la inyección colisional y el efecto Yarkovsky, analizar la mezcla de material entre las diferentes regiones del Cinturón Principal, y determinar el origen de los NEAs, nuestro modelo divide el cinturón de asteroides en tres regiones de semieje mayor:

- el Anillo Interior (AI), entre las resonancias $\nu_{6}$ y 3:1, desde $\sim 2$ a $2.5 \mathrm{UA}$,

- el Anillo Medio (AM), entre las resonancias 3:1 y 5:2, desde 2.5 a $2.82 \mathrm{UA}$, y

- el Anillo Exterior (AE), entre las resonancias 5:2 y 2:1, desde 2.82 a 3.27 UA.

Con el objetivo de simular la evolución colisional y dinámica de las distribuciones de tamaños del Cinturón Principal de asteroides y la población de NEAs, nuestro código numérico evoluciona en el tiempo el número de cuerpos asociados con cada uno de los tres anillos del cinturón de asteroides y la población de NEAs. Las poblaciones de objetos residen en un conjunto de 130 bines de tamaño, cuyos valores centrales se ubican en el rango que va desde $D_{1}=10^{-10} \mathrm{~km}$ a $D_{130}=886.7 \mathrm{~km}$ de diámetro en una forma tal que desde un bin al siguiente, la masa del cuerpo cambia en un factor 2 mientras que su diámetro se modifica por un factor $2^{1 / 3}$. Para construir estas poblaciones, adoptamos una densidad de $2.7 \mathrm{~g} \mathrm{~cm}^{-3}$, la cual es consistente con aquello discutido en la Sección 3.2.1.

Mientras que la población de NEAs siempre comienza con una masa inicial igual a cero, la masa total de los objetos asociados con cada uno de los tres anillos del Cinturón Principal de asteroides es calculada a partir del modelo de distribución de masa en la nebulosa planetaria propuesto por Weidenschilling (1977). De acuerdo con esto, adoptamos una densidad superficial $\Sigma$ del disco nebular de la forma

$$
\Sigma(a)=\Sigma_{0}\left(\frac{a}{a_{0}}\right)^{-3 / 2},
$$




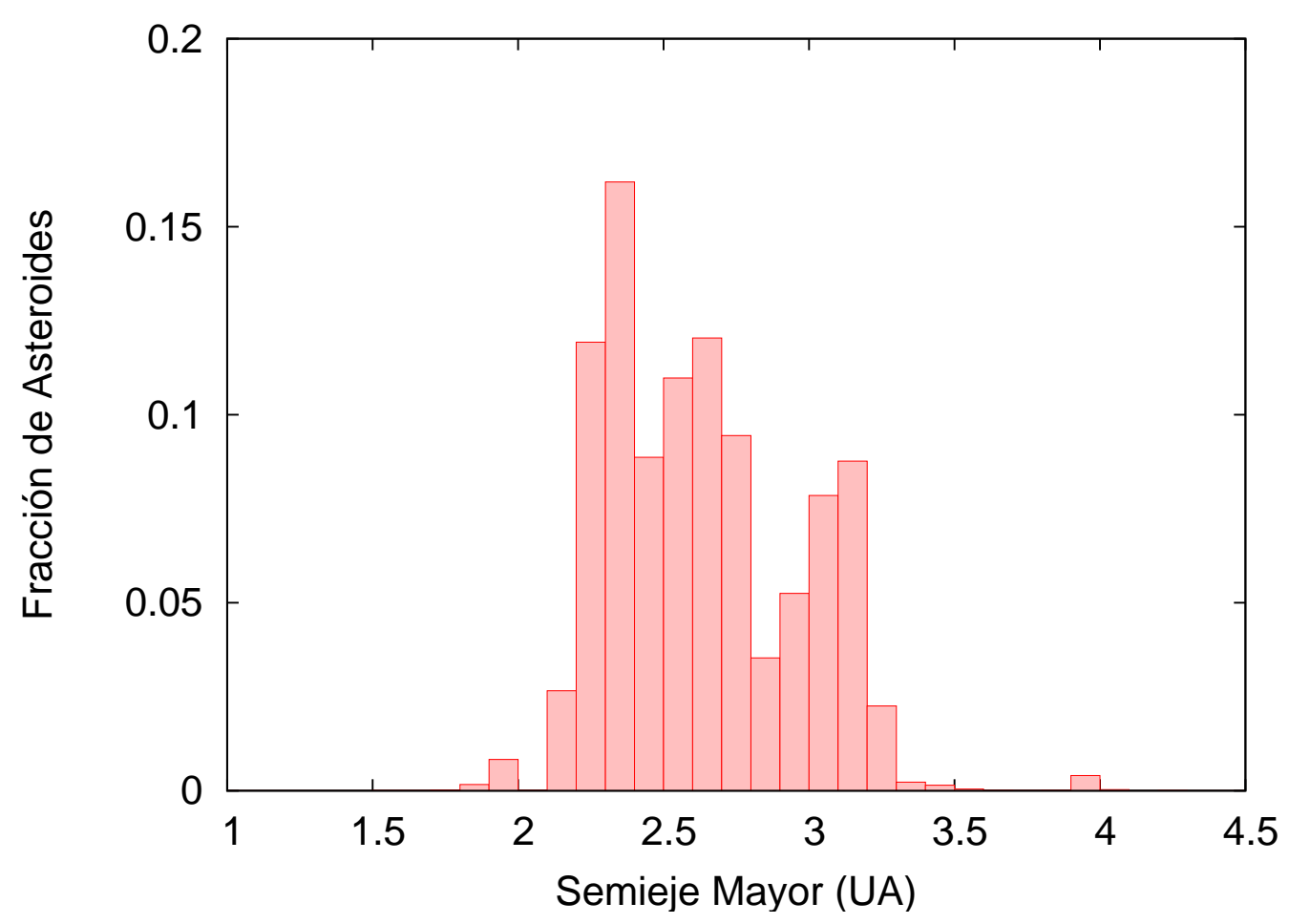

Figura 5.1. Histograma de asteroides en función del semieje mayor. Entre las 3.27 y 3.4 UA se ubica menos del $2 \%$ de la población del Cinturón Principal de asteroides.

donde $\Sigma_{0}$ es el valor asociado a un radio arbitrario $a_{0}$. De este modo, la masa diferencial $d M(a)$ contenida en un anillo de radio $a$ y ancho $d a$ está dada por

$$
\begin{aligned}
d M(a) & =2 \pi a \Sigma(a) d a \\
& =2 \pi a \Sigma_{0}\left(\frac{a}{a_{0}}\right)^{-3 / 2} d a,
\end{aligned}
$$

y a partir de esto, la masa del Cinturón Principal completo es

$$
\begin{aligned}
M_{\mathrm{MB}} & =2 \pi \Sigma_{0} a_{0}^{3 / 2} \int_{2}^{3,27} a^{-1 / 2} d a \\
& =M_{0} \int_{2}^{3,27} a^{-1 / 2} d a .
\end{aligned}
$$

De la misma forma, las masas asociadas con cada uno de los tres anillos del Cinturón Principal de asteroides están dadas por

$$
\begin{aligned}
M_{\mathrm{AI}} & =M_{0} \int_{2}^{2,5} a^{-1 / 2} d a, \\
M_{\mathrm{AM}} & =M_{0} \int_{2,5}^{2,82} a^{-1 / 2} d a, \\
M_{\mathrm{AE}} & =M_{0} \int_{2,82}^{3,27} a^{-1 / 2} d a .
\end{aligned}
$$


Proponiendo una dada masa para el cinturón de asteroides inicial, es posible obtener la constante $M_{0}$ a partir de la Ecuación 5.3, y luego determinar las masas correspondientes para los anillos Interior, Medio y Exterior, dadas por las Ecuaciones 5.4. La masa actual contenida en el Cinturón Principal de asteroides es de $\sim 5 \times 10^{-4} \mathrm{M}_{\oplus}$. Si bien nosotros testeamos diferentes masas iniciales, los resultados mostrados aquí son aquellos obtenidos considerando un cinturón inicial con $\sim 7$ veces la masa del Cinturón Principal actual, esto es, $\sim 3.5 \times 10^{-3}$ $\mathrm{M}_{\oplus}$.

Una vez que las masas asociadas con los anillos Interior, Medio y Exterior del Cinturón Principal de asteroides son determinadas, el siguiente paso es construir las poblaciones iniciales para cada uno de estos anillos. Las mismas son definidas de la siguiente manera:

- Anillo Interior $(2<a<2.5 \mathrm{UA})$ : para $D \gtrsim 150 \mathrm{~km}$, asumimos que el número de objetos es igual al número de asteroides observados en esta región del Cinturón Principal. Para $80 \lesssim D \lesssim 150 \mathrm{~km}$, usamos una ley de potencia de la forma $C D^{q}$, con una valor de $q$ de $\sim-1.6$. Para $D \lesssim 80 \mathrm{~km}$, adoptamos la misma ley de potencias propuesta anteriormente aunque con valor de $q$ de $\sim-3.1$ (Figura 5.2a).

- Anillo Medio $(2.5<a<2.82 \mathrm{UA})$ : Para $D \gtrsim 250 \mathrm{~km}$, asumimos que el número de objetos es igual al número de asteroides observados en esta región del Cinturón Principal. Para $100 \lesssim D \lesssim 250 \mathrm{~km}$, usamos una ley de potencia de la forma $C D^{q}$, con una valor de $q$ de $\sim-2.2$. Para $D \lesssim 100 \mathrm{~km}$, adoptamos la misma ley de potencias propuesta anteriormente aunque con valor de $q$ de $\sim-2.9$ (Figura 5.2b).

- Anillo Exterior $(2.82<a<3.27 \mathrm{UA})$ : Para $D \gtrsim 350 \mathrm{~km}$, asumimos que el número de objetos es igual al número de asteroides observados en esta región del Cinturón Principal. Para $120 \lesssim D \lesssim 350 \mathrm{~km}$, usamos una ley de potencia de la forma $C D^{q}$, con una valor de $q$ de $\sim-3.3$. Para $D \lesssim 120 \mathrm{~km}$, adoptamos la misma ley de potencias propuesta anteriormente aunque con valor de $q$ de $\sim-2.9$ (Figura 5.2c).

Estas poblaciones iniciales son construidas siguiendo la idea propuesta por Bottke et al. (2005a) para el Cinturón Principal completo. En efecto, estos autores construyeron las poblaciones iniciales de su modelo asumiendo que, para $D>D_{1}=200 \mathrm{~km}$, el número de objetos es aproximadamente igual al número de asteroides observados en el Cinturón Principal, mientras que para $D_{2}<D<D_{1}=200 \mathrm{~km}$, donde $D_{2}$ es $\sim 100 \mathrm{~km}$, la población sigue una ley de potencias de la forma $C D^{q}$, con una valor de $q$ cercano a la pendiente determinada por los asteroides observados en este rango de tamaños. Aquí, asumimos valores para $D_{1}$ y $D_{2}$ de 150 y $80 \mathrm{~km}, 250$ y $100 \mathrm{~km}$, y 350 y $120 \mathrm{~km}$ para los Anillos Interior, Medio y Exterior, respectivamente. Luego, para $D<D_{2}$, usamos la misma ley de potencias definida anteriormente para cada uno de los tres anillos del Cinturón Principal aunque con un valor de $q$ determinado para reproducir sus masas asociadas. A partir de la combinación de estas tres poblaciones, es posible determinar la población inicial asociada con el Cinturón Principal completo. En efecto, para $D \gtrsim 350 \mathrm{~km}$, la población inicial resultante tiene un número de cuerpos igual al número de asteroides observados en el cinturón de asteroides entero. Además, para $200 \lesssim D \lesssim 350 \mathrm{~km}$, obtenemos una ley de potencias de la forma $C D^{q}$, con un valor de $q$ de $\sim-5$. Para $D \lesssim 200 \mathrm{~km}$, la población resultante también es descripta por una ley de potencias análoga a la propuesta anteriormente, aunque con un valor de $q$ de $\sim-2.9$ (Figura 5.2d). Estas poblaciones iniciales son consistentes con el trabajo de Bottke et al. (2005a), el cual indica 

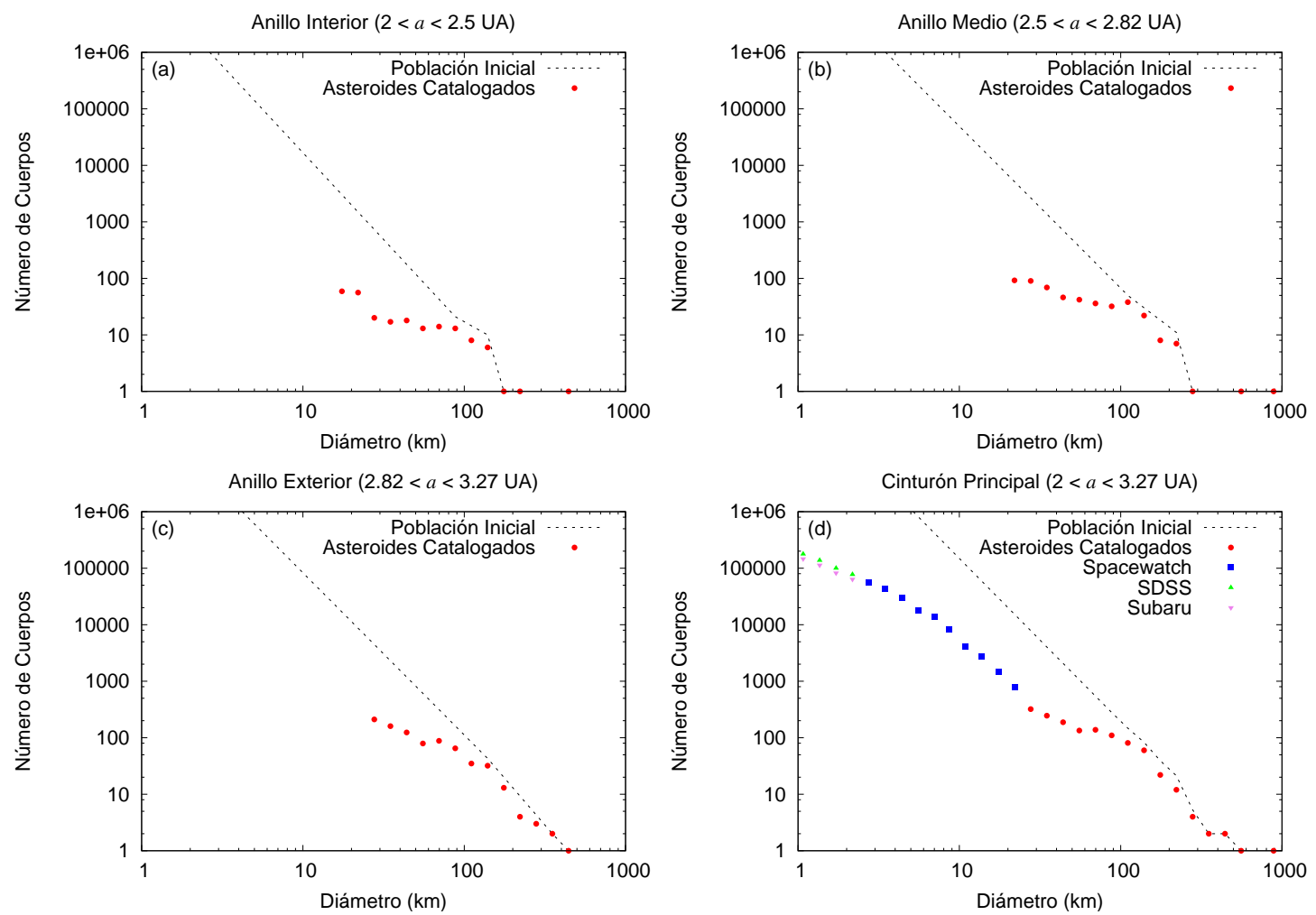

Figura 5.2. Poblaciones iniciales del modelo asociadas a los Anillos Interior a), Medio b) y Exterior c) y al Cinturón Principal completo d).

que no es posible reproducir las distintas estructuras de onda en la distribución de tamaños del Cinturón Principal a partir de una población inicial representada por una ley de potencias con una única pendiente.

Por otra parte, las simulaciones numéricas desarrolladas por Petit et al. (1999) y Petit et al. (2001) sugieren que el Cinturón Principal de asteroides podría haber contenido originalmente cientos de veces más masa que aquella que posee actualmente. Además, dichos autores indicaron que las perturbaciones gravitacionales de Júpiter y embriones planetarios primordiales redujeron muy rápidamente la masa del cinturón inicial, alcanzando su valor actual sobre escalas de tiempo de unos pocos millones de años. Para tener en cuenta este resultado y siguiendo la idea propuesta por O'Brien \& Greenberg (2005), las poblaciones iniciales asociadas con cada uno de los tres anillos del Cinturón Principal son inicialmente multiplicadas por un dado factor y su evolución seguida por $5 \times 10^{6}$ años. Luego, las poblaciones residuales son reducidas por el mismo factor y finalmente su evolución es analizada por el resto de los 4.5 $\times 10^{9}$ años. Nosotros desarrollamos una serie de simulaciones numéricas testeando diferentes valores para este factor, sin obtener cambios relevantes en los resultados. En la Sección 5.7, se exponen los resultados obtenidos utilizando un factor con un valor igual a 55. A partir de esto, se obtiene una población inicial para el Cinturón Principal completo cuya masa es de 385 veces la masa del cinturón de asteroides actual, lo cual es consistente con los resultados obtenidos por Petit et al. (1999) y Petit et al. (2001) para el cinturón primitivo. 


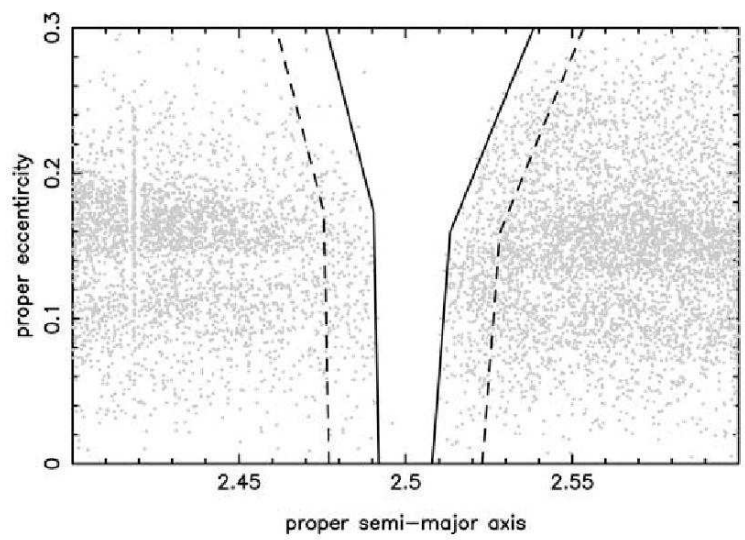

(a)

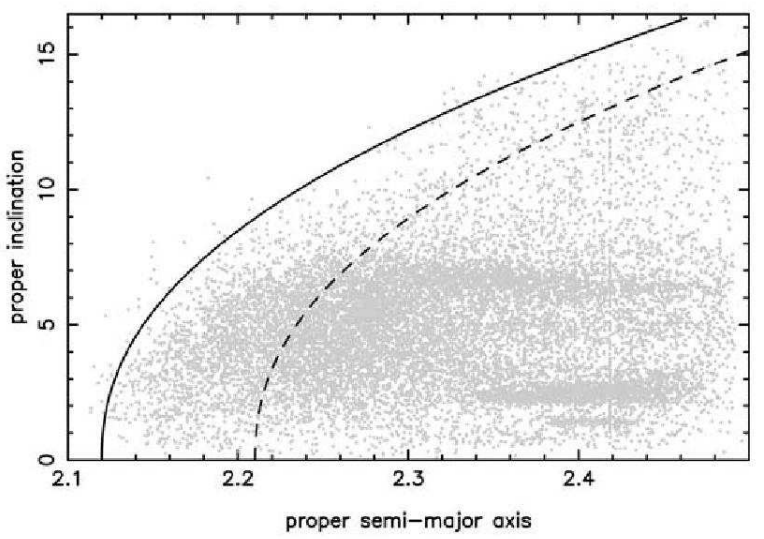

(b)

Figura 5.3. Distribución de asteroides con respecto a los elementos propios semieje mayor y excentricidad, en la vecindad de la resonancia de movimientos medios 3:1 con Júpiter a) y de la resonancia secular $\nu_{6}$ b). En a) y b), los límites de estas resonancia, computados por las Ecuaciones 5.5 y 5.6 para la $3: 1$ y por la Ecuación 5.7 para la $\nu_{6}$, son representados por líneas sólidas. Además, los límites modificados con el objetivo de computar el flujo efectivo de asteroides dentro de estas regiones resonantes son representados por líneas de rayas. Figura extraída de Morbidelli \& Vokrouhlický (2003).

\subsection{Límites de las Resonancias $\nu_{6}, 3: 1$ y 5:2}

Tal como indicamos en la Sección 3.1.2, los objetos pertenecientes a la resonancia secular $\nu_{6}$ y la resonancia de movimientos medios 3:1 con Júpiter son removidos sobre escalas de tiempo de $2 \times 10^{6}$ años, mientras que la resonancia de movimientos medios 5:2 con Júpiter remueve sus cuerpos residentes en sólo $5 \times 10^{5}$ años. Como consecuencia de esto, las resonancias $\nu_{6}, 3: 1$ y 5:2 muestran gaps bien definidos en la distribución de los asteroides del Cinturón Principal. Para las resonancias 3:1 y 5:2, los límites de estos gaps dependen de la excentricidad orbital mientras que, para la resonancia $\nu_{6}$, los mismos dependen de la inclinación orbital y sólo débilmente de la excentricidad (Morbidelli \& Henrard 1991).

Para definir los límites de las resonancias $\nu_{6}$ y 3:1, Morbidelli \& Vokrouhlický (2003) hicieron uso del catálogo de elementos propios $^{1}$ desarrollado por Knežević \& Milani (2000). Estudiando la distribución de los asteroides de este catálogo en el espacio de los elementos propios semieje mayor $a$, excentricidad $e$ e inclinación $i$, Morbidelli \& Vokrouhlický (2003) encontraron que los bordes de los gaps asociados a las resonancias $3: 1$ y $\nu_{6}$ pueden ser aproximados por las siguientes expresiones:

$$
\begin{array}{lll}
a=2.508+\frac{e}{29.615} & \text { para } & e \leq 0.15936, \\
a=2.485+\frac{e}{5.615} & \text { para } & e>0.15936,
\end{array}
$$

\footnotetext{
${ }^{1}$ Los elementos propios eliminan las oscilaciones de corto y largo período experimentadas por los elementos orbitales instantáneos como consecuencia de las perturbaciones planetarias. A partir de esto, el uso de los elementos propios permite una buena visualización de los gaps asociados a las resonancias poderosas. Por el contrario, los elementos instantáneos no resultan ser adecuados, ya que al utilizarlos como herramienta de análisis, los asteroides pueden temporariamente invadir los gaps debido a las oscilaciones de corto período de sus semiejes mayores.
} 


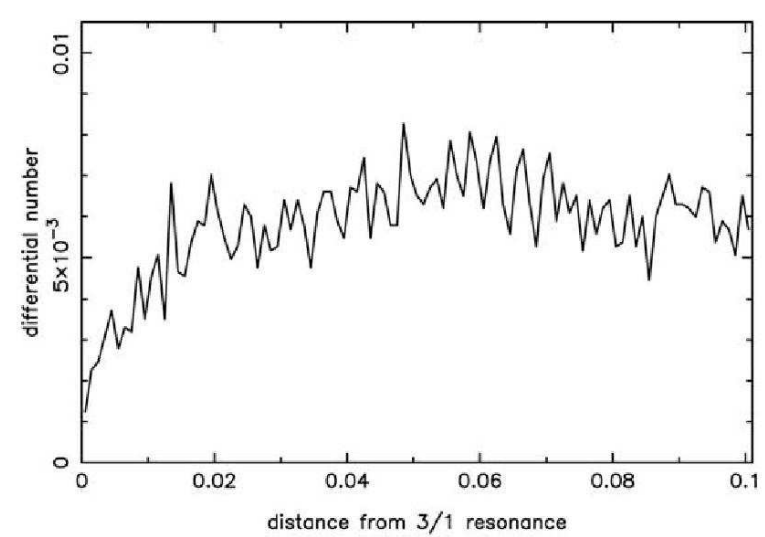

(a)

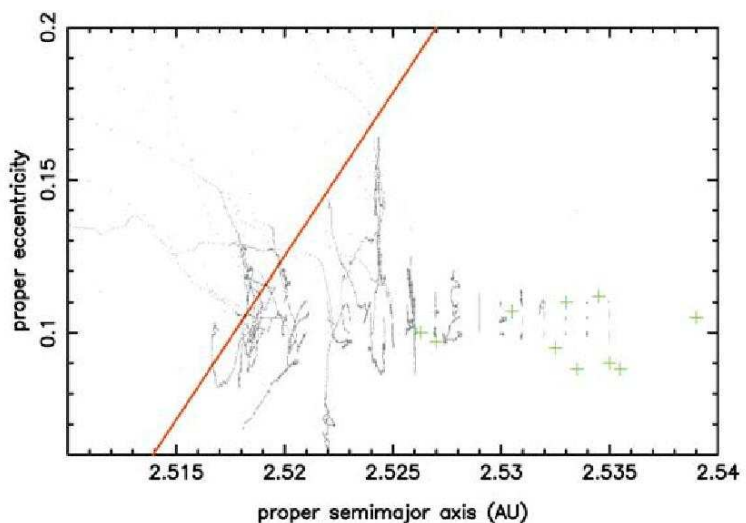

(b)

Figura 5.4. a) Número de asteroides como función de la distancia al borde derecho de la resonancia 3:1. La curva muestra que el número de objetos crece hasta $\sim 0.015$ UA medidas desde el borde de la resonancia, permaneciendo más o menos constantes sobre las siguientes 0.025 UA. b) Evolución de 45 partículas en el espacio de los elementos propios (a,e), durante $10^{8}$ años. El proceso de difusión caótica remueve fácilmente los objetos localizados dentro de las 0.0075 UA del borde derecho de la resonancia, el cual está representado por la línea roja. Figura extraída de Morbidelli \& Vokrouhlický (2003).

para el costado derecho de la resonancia 3:1,

$$
\begin{aligned}
& a=2.492-\frac{e}{108.85} \quad \text { para } \quad e \leq 0.1734 \text {, } \\
& a=2.51-\frac{e}{8.85} \quad \text { para } \quad e>0.1734 \text {, }
\end{aligned}
$$

para el costado izquierdo de la resonancia $3: 1, y$

$$
a=2.12+6.003(\sin i)^{2.256}
$$

para el costado derecho de la resonancia $\nu_{6}$.

Las Figuras 5.3 y 5.4 pertenecen al trabajo de Morbidelli \& Vokrouhlický (2003). La Figura 5.3a muestra la distribución de los asteroides en el espacio de elementos propios alrededor de la resonancia 3:1. Los bordes de esta resonancia, definidos por las Ecuaciones 5.5 y 5.6, son ilustrados a través de las líneas sólidas. Dicha figura sugiere que la densidad de asteroides decae abruptamente a medida que nos alejamos de los bordes de la resonancia 3:1. Este efecto puede ser mejor visualizado en la Figura 5.4a, la cual expone el número de asteroides del catálogo de elementos propios como una función de la distancia al borde derecho de dicha resonancia. La distancia es computada como la diferencia entre $a^{\prime}$ y $a$, donde $a^{\prime}$ es el semieje mayor propio del asteroide, y a está dado por la Ecuación 5.5 usando como valor de $e$ a la excentricidad propia del objeto. Notemos que el número de asteroides se incrementa fuertemente hasta las $\sim 0.015 \mathrm{UA}$ medidas desde el borde de la resonancia, y luego resulta más o menos constante sobre las 0.025 UA siguientes. Guillens et al. (2002) mostraron que la dinámica en la vecindad de la resonancia 3:1 resulta ser débilmente caótica, justificando esta remoción parcial de asteroides en los bordes de la misma. En efecto, como consecuencia de la difusión caótica, los asteroides localizados entre 0.01 y 0.02 UA de los bordes de la 
resonancia tienden a migrar en el espacio de los elementos propios, cayendo a la resonancia en una escala de tiempo de $\sim 10^{8}$ años. Este fenómeno es ilustrado en la Figura 5.4b, la cual expone la evolución de 45 partículas en el espacio de los elementos propios $(a, e)$, durante $10^{8}$ años. A partir de la misma se observa que los objetos localizados dentro de las 0.0075 UA del borde derecho de la resonancia (línea roja) son fácilmente removidos debido al proceso de difusión caótica. La razón por la cual los bordes de la resonancia 3:1 no se encuentran completamente despoblados de asteroides también fue discutida por Guillens et al. (2002), quienes argumentaron que el efecto Yarkovsky juega el rol clave, resuministrando continuamente estas regiones con nuevos cuerpos. Tal como fue indicado en la Sección 3.1.2, el efecto Yarkovsky modifica el semieje mayor de los asteroides, haciéndolos migrar por diferentes regiones del Cinturón Principal. Una vez que estos cuerpos alcanzan la región difusiva de la resonancia 3:1, el proceso de difusión caótica comienza a ser importante, removiéndolos sobre escalas de tiempo comparables o menores a aquellas que se requerirían si el efecto Yarkovsky tuviese que arrastrarlos hasta los bordes de la resonancia. El abrupto incremento en el número de asteroides en la vecindad de la resonancia 3:1 es el resultado de este mecanismo.

Para evaluar correctamente el número de asteroides que ingresan en la resonancia 3:1, Morbidelli \& Vokrouhlický (2003) midieron el flujo de objetos dentro de la región difusiva de la resonancia y no dentro de los límites delineados por el gap. Para hacer esto, aquellos autores modificaron los límites de la resonancia 3:1, definiendo los mismos 0.015 UA a la derecha y a la izquierda de los bordes dados por las Ecuaciones 5.5 y 5.6, respectivamente. Estos límites efectivos son ilustrados en la Figura 5.3a a través de las líneas de rayas. En este escenario, Morbidelli \& Vokrouhlický (2003) despreciaron los posibles procesos colisionales en la zona difusiva, asumiendo que todos los cuerpos que ingresan a dicha región son capturados eventualmente dentro de la resonancia. Los autores establecen que esta no debería ser una simplificación severa del modelo, ya que los tiempos de vida colisionales medios para objetos de 1 y $10 \mathrm{~km}$ de tamaño son de, aproximadamente, $3.5 \times 10^{8}$ y $1.2 \times 10^{9}$ años, respectivamente. De acuerdo con esto, los procesos colisionales pueden ser despreciados en este tratamiento ya que los mismos actúan sobre escalas de tiempo mayores a los $\sim 10^{8}$ años requeridos para la remoción de objetos de la zona difusiva.

Por otra parte, la Figura 5.3b muestra la distribución de los asteroides en el espacio de elementos propios alrededor de la resonancia secular $\nu_{6}$. El borde derecho de esta resonancia, definido por la Ecuación 5.7, es ilustrado a través de la curva sólida. A partir de dicha figura resulta evidente que la densidad de asteroides decae a medida que nos alejamos del borde de la resonancia, sugiriendo la existencia significativa de procesos de difusión caótica similares a aquellos descriptos para la resonancia 3:1. Morbidelli \& Gladman (1998) y Nesvorný et al. (2002b) estudiaron la estructura dinámica de la resonancia secular $\nu_{6}$ a partir de simulaciones numéricas. Estos trabajos indican que la difusión caótica resulta ser efectiva dentro de las 0.1 UA del borde de la resonancia. En efecto, los análisis establecen que el número de cuerpos se incrementa fuertemente hasta las 0.08-0.09 UA en la vecindad de la misma. Para tener en cuenta el fenómeno de difusión y evaluar correctamente el número de asteroides que ingresan en la resonancia $\nu_{6}$, Morbidelli \& Vokrouhlický (2003) procedieron de un modo similar a lo realizado para la resonancia 3:1. En efecto, aquellos autores modificaron el límite de la resonancia $\nu_{6}$, definiendo el mismo 0.09 UA hacia la derecha del borde dado por la Ecuación 5.7. Este límite efectivo es ilustrado en la Figura 5.3b a través de la curva de rayas.

Para definir los límites de la resonancia 5:2, nosotros desarrollamos un análisis similar a aquel realizado por Morbidelli \& Vokrouhlický (2003) sobre las resonancias $\nu_{6}$ y 3:1. Usando 

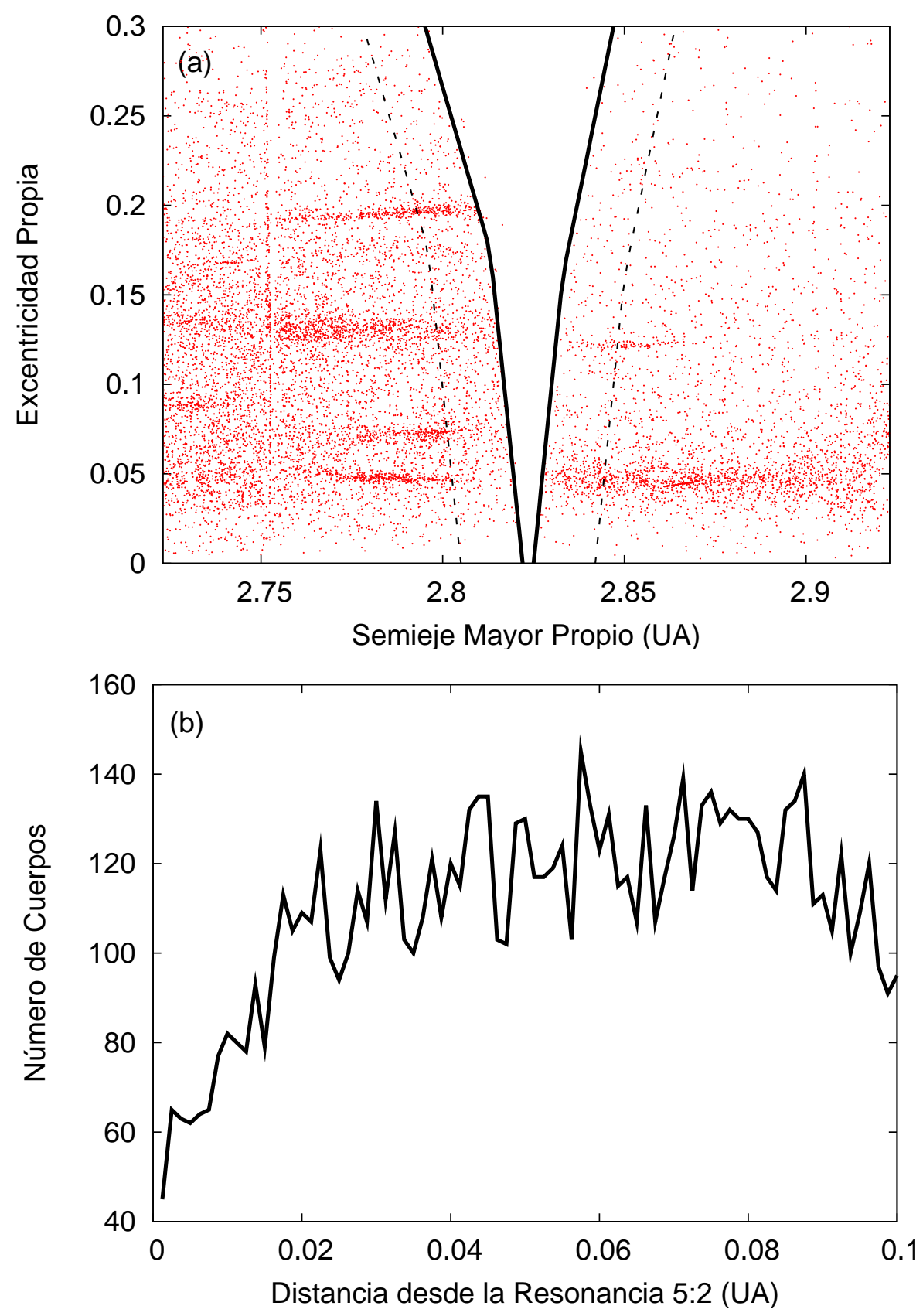

Figura 5.5. a) Distribución de los asteroides con respecto a los elementos propios semieje mayor y excentricidad, en la vecindad de la resonancia de movimientos medios 5:2 con Júpiter. Los límites derecho e izquierdo de esta resonancia, aproximados por las Ecuaciones 5.8 y 5.9, respectivamente, son representados por líneas sólidas. Los límites modificados con el objetivo de computar el flujo efectivo de asteroides dentro de esta región resonante son representados por las líneas de rayas. b) El número de asteroides como función de la distancia desde el borde izquierdo de la resonancia 5:2. La curva muestra que la densidad de objetos crece hasta $\sim 0.017$ UA de la resonancia, permaneciendo más o menos constantes sobre las siguientes $0.03 \mathrm{UA}$. 
el catálogo de elementos propios de Knežević \& Milani (2000), es posible observar un gap bien definido en la resonancia 5:2, el cual es ilustrado en la Figura 5.5a. De acuerdo a nuestro estudio, los límites de esta resonancia pueden ser aproximados por las siguientes expresiones

$$
\begin{array}{lll}
a=2.825+\frac{e}{20} & \text { para } & e \leq 0.16, \\
a=2.817+\frac{e}{10} & \text { para } & e>0.16,
\end{array}
$$

para el borde derecho, y

$$
\begin{aligned}
& a=2.822-\frac{e}{19.5} \quad \text { para } \quad e \leq 0.17, \\
& a=2.838-\frac{e}{7} \quad \text { para } \quad e>0.17,
\end{aligned}
$$

para el borde izquierdo. Estos límites son ilustrados en la Figura 5.5a a través de las líneas sólidas.

De la Figura 5.5a resulta evidente que la densidad de asteroides decrece a medida que nos alejamos de los bordes de la resonancia, lo cual es debido a fenómenos de difusión caótica similares a aquellos descriptos para las resonancias $\nu_{6}$ y $3: 1$. Debido a la difusión en la vecindad de sus bordes, es necesario redefinir los límites de la resonancia 5:2. Nuestro análisis nos muestra que el número de asteroides crece hasta $\sim 0.015-0.017$ UA desde el borde izquierdo de la resonancia 5:2 y luego se mantiene más o menos constante sobre las siguientes 0.03 UA (Figura 5.5b). Con el objetivo de computar el flujo efectivo de asteroides que ingresan en la resonancia 5:2, modificamos los límites de esta región resonante, definiendo los mismos 0.017 UA a la derecha y a la izquierda de los bordes dados por las Ecuaciones 5.8 y 5.9, respectivamente. Estos límites efectivos son ilustrados en la Figura 5.5a a través de las líneas de rayas.

De aquí en más, los límites efectivos, tal como fueron modificados con el fin de computar correctamente el flujo de asteroides dentro de las resonancias $\nu_{6}, 3: 1$ y 5:2, son aquellos con los cuales desarrollaremos nuestro trabajo.

\subsection{El Rol del Efecto Yarkovsky}

\subsubsection{Consideraciones Generales}

El efecto Yarkovsky es el resultado de un mecanismo de radiación que puede causar cambios relevantes en los parámetros orbitales de los cuerpos rotantes del Sistema Solar, debido a la asimetría existente entre la dirección de absorción de la luz del Sol y la dirección de reemisión de la radiación térmica. Existen dos variantes bien diferenciadas de este mecanismo para un cuerpo rotante en órbita alrededor del Sol:

- el efecto diurno debido a su movimiento de rotación,

- el efecto estacional debido a su movimiento orbital.

El efecto Yarkovsky diurno se produce debido a que un cuerpo rotante que absorbe radiación del Sol rota antes de que dicha energía sea reemitida como radiación térmica infrarroja. Este 
mecanismo lleva a una asimetría longitudinal entre la dirección de absorción de la luz del Sol y la dirección de reemisión, la cual genera una fuerza en la dirección de movimiento del cuerpo. Dicha fuerza tiene el sentido del movimiento orbital si la rotación del objeto se produce en sentido directo, y tiene sentido contrario si el mismo rota en sentido retrógrado. A partir de esto, el efecto diurno causa un incremento en el semieje mayor de la órbita cuando el objeto rota en sentido directo, y un decrecimiento si la rotación es retrógrada. Esta variante diurna resulta ser máxima cuando el eje de rotación del objeto es perpendicular al plano orbital, mientras que su efectividad se hace nula para una oblicuidad de $90^{\circ}$. La Figura 5.6a muestra la fuerza generada por el efecto Yarkovsky diurno para el caso de rotación directa y oblicuidad nula.

El efecto Yarkovsky estacional se produce debido a que un cuerpo que absorbe radiación del Sol se mueve en su órbita antes de que esta energía sea reemitida como radiación térmica infrarroja. Este mecanismo lleva a una asimetría latitudinal entre la dirección de absorción de la luz del Sol y la dirección de reemisión, la cual genera una fuerza que es siempre opuesta a la dirección del movimiento orbital. Como consecuencia de esto, el efecto estacional siempre causa un decrecimiento en el semieje mayor de la órbita. Esta variante estacional alcanza su máximo valor para una oblicuidad de $90^{\circ}$, y tiene magnitud cero para una oblicuidad nula. La Figura 5.6b ilustra la fuerza generada por el efecto Yarkovsky estacional para una oblicuidad de $90^{\circ}$.

El efecto Yarkovsky es dependiente del tamaño y afecta fundamentalmente los parámetros orbitales de los pequeños asteroides, con tamaños inferiores al kilómetro. Por el contrario, los objetos mayores prácticamente no resultan afectados. Debido a esta dependencia con el tamaño, el efecto Yarkovsky puede influir significativamente en la distribución de tamaños de los asteroides del Cinturón Principal. Además, ya que se trata de un mecanismo de radiación, la eficiencia de este efecto decrece con un incremento del semieje mayor.

En los últimos años, diversos trabajos fueron desarrollados con el objetivo de estudiar este mecanismo. Farinella et al. (1998) llevaron a cabo un modelo del efecto Yarkovsky considerando tanto la variante diurna como la variante estacional, y obtuvieron expresiones explícitas para la tasa de cambio del semieje mayor. Tiempo después, Penco et al. (2002) incluyeron el efecto Yarkovsky en modelos numéricos destinados a estudiar la evolución colisional de los asteroides del Cinturón Principal. Más tarde, Morbidelli \& Vokrouhlický (2003) desarrollaron simulaciones con el objetivo de estudiar el rol del efecto Yarkovsky en el origen de la población de NEAs. Este trabajo indica que el efecto Yarkovsky es el mecanismo principal por el cual los asteroides son continuamente inyectados dentro de las resonancias poderosas y difusivas del Cinturón Principal, manteniendo la población de NEAs en un estado estacionario. Estas conclusiones nos conducen a inferir que este mecanismo de radiación junto con las rutas de escape resonantes representan una importante fuente de pérdida de masa en el Cinturón Principal de asteroides.

Existen diversos mecanismos que pueden modificar la magnitud del efecto Yarkovsky. El efecto YORP (Yarkovsky-O'Keefe-Radzievskii-Paddack) y las reorientaciones colisionales de los ejes de rotación de los asteroides pueden producir cambios en la oblicuidad de tales objetos, llevando a sus semiejes a variar de modo aleatorio en lugar de experimentar un cambio suave y continuo. El efecto YORP es el resultado de un mecanismo de radiación que puede modificar tanto la tasa de rotación como la oblicuidad de los asteroides (Rubincam et al. 2002; Vokrouhlický \& Čapek 2002). Una discusión interesante concerniente a este efecto puede ser 


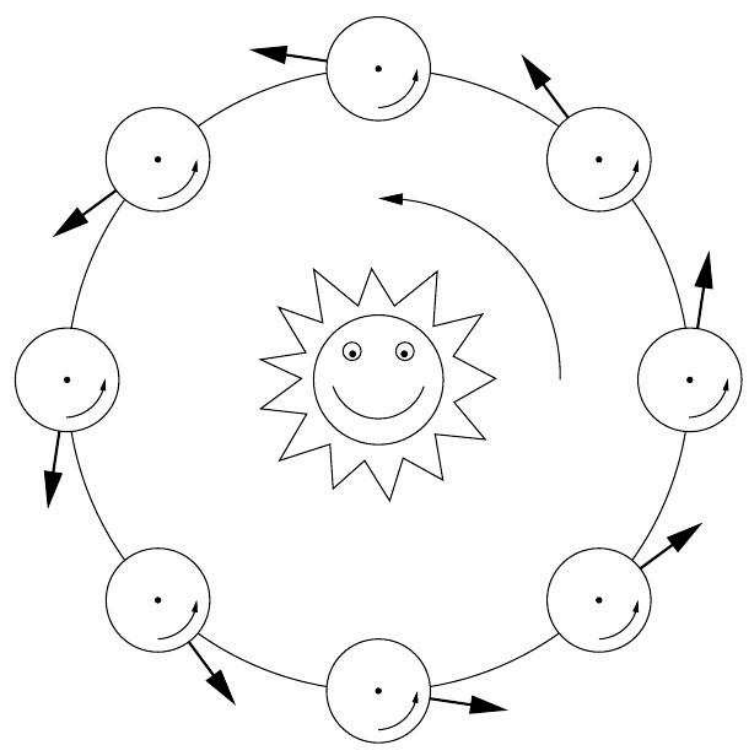

(a)

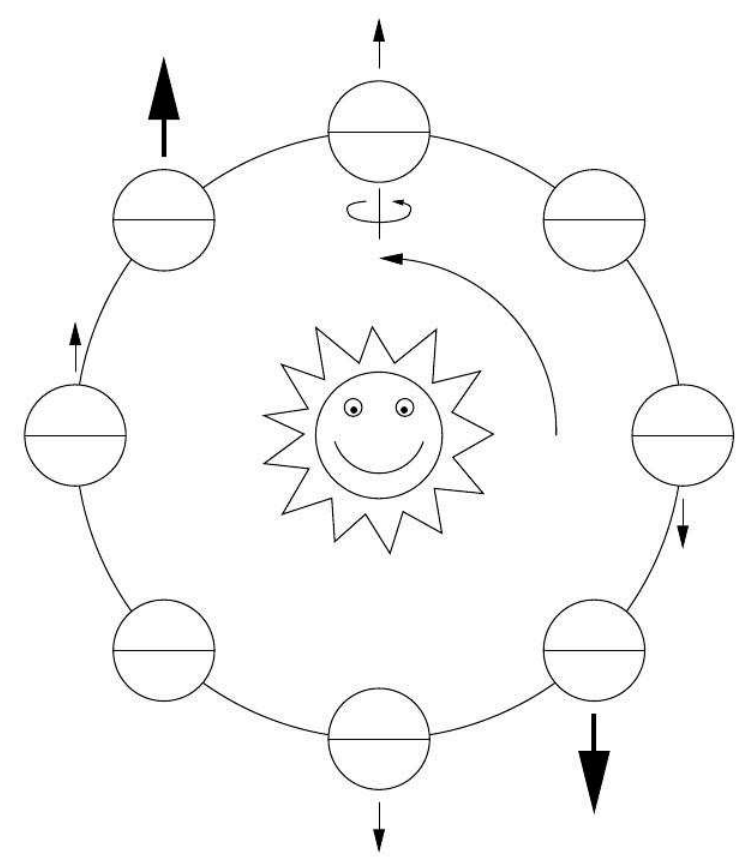

(b)

Figura 5.6. a) Ilustración del efecto Yarkovsky diurno para el caso de oblicuidad nulo y rotación directa. Debido a la rotación del objeto, existe una asimetría entre la dirección de absorción de la luz del Sol y la dirección de reemisión. Esta asimetría genera una fuerza (cuya dirección y sentido se indican a través de las flechas) que causa un incremento en el semieje mayor de la órbita del cuerpo. En el caso de rotación retrógrada, el semieje mayor sufrirá un decrecimiento. b) Ilustración del efecto Yarkovsky estacional para una oblicuidad de $90^{\circ}$. Debido al movimiento del cuerpo a lo largo de su órbita, existe una asimetría entre la dirección de absorción de la luz del Sol y la dirección de reemisión. Esta asimetría genera una fuerza (cuya dirección y sentido se indican a través de las flechas) que causa un decrecimiento en el semieje mayor de la órbita del cuerpo. Figura extraída de O’Brien \& Greenberg (2005). 
encontrada en Morbidelli \& Vokrouhlický (2003).

\subsubsection{Modelo Matemático}

Con el objetivo de calcular la tasa de remoción de objetos desde cada uno de los tres anillos del Cinturón Principal de asteroides debido a la acción del efecto Yarkovsky y las resonancias orbitales, usamos las expresiones derivadas por O'Brien \& Greenberg (2005), las cuales están basadas en el modelo analítico delineado por Farinella et al. (1998). Aquí, presentamos una breve descripción matemática de este efecto, considerando individualmente los tratamientos desarrollados para estudiar las variantes diurna y estacional de este mecanismo de radiación.

\section{Efecto Yarkovsky Diurno}

De acuerdo al trabajo de Farinella et al. (1998), si $F_{\mathrm{Y}}$ es la fuerza por unidad de masa del cuerpo generada por el efecto Yarkovsky, el cambio en su semieje mayor está dado por la siguiente expresión:

$$
\dot{a}=\frac{2 F_{\mathrm{Y}}}{n},
$$

donde $n=2 \pi / P_{\text {orb }}$ es el movimiento orbital medio y, de acuerdo a lo discutido por Burns et al. (1979), $F_{\mathrm{Y}}$ puede ser expresada por la fórmula

$$
F_{\mathrm{Y}}=\frac{2}{\rho R} \frac{\epsilon \sigma T^{4}}{c} \frac{\Delta T}{T} f(\zeta)
$$

donde $\rho$ es la densidad del cuerpo, $R$ es su radio, $\epsilon$ es la emisividad infrarroja superficial (la cual asumimos igual a la unidad), $\sigma=5.67 \times 10^{-8} \mathrm{~W} \mathrm{~m}^{-2} \mathrm{~K}^{-4}$ es la constante de StefanBoltzmann, $c=299792458 \mathrm{~m} \mathrm{~s}^{-1}$ es la velocidad de la luz, $T$ es la temperatura efectiva del cuerpo, $\Delta T$ es la diferencia de temperatura entre el hemisferio iluminado y el hemisferio obscuro del objeto, y $f(\zeta)$ es la función oblicuidad. La temperatura efectiva del cuerpo puede ser calculada igualando la fracción de flujo solar absorbida al flujo emitido por el asteroide. A partir de esto

$$
\pi R^{2}(1-A) S=4 \pi R^{2} \epsilon \sigma T^{4}
$$

donde $A$ es el albedo (al cual asumimos igual a 0 ) y $S$ es el flujo solar en la posición del cuerpo. $S$ depende del semieje mayor y puede ser expresado por la fórmula

$$
S=S_{0}\left(\frac{a_{0}}{a}\right)^{2}
$$

donde $S_{0}=1370 \mathrm{~W} \mathrm{~m}^{-2}$ es la constante solar y $a_{0}$ y $a$ son los semiejes mayores de la Tierra y el objeto bajo consideración, respectivamente. Finalmente, la temperatura efectiva está dada por la siguiente expresión

$$
T=\left\{\frac{(1-A) S}{4 \epsilon \sigma}\right\}^{1 / 4} .
$$

La variante diurna representa el caso más simple del efecto Yarkovsky. De acuerdo a la Ecuación 5.11, la efectividad de este mecanismo resulta ser directamente proporcional a $\Delta T$. Cuando el calentamiento sobre un costado del cuerpo comienza a afectar inmediatamente su 


\begin{tabular}{lccc}
\hline Material & $\boldsymbol{\rho}\left(\mathrm{kg} \mathrm{m}^{-\mathbf{3}}\right)$ & $\boldsymbol{K}\left(\mathbf{W ~ m}^{-\mathbf{1}} \mathbf{K}^{-\mathbf{1}}\right)$ & $\boldsymbol{C}\left(\mathbf{J ~ k g}^{-\mathbf{1}} \mathbf{K}^{-\mathbf{1}}\right)$ \\
\hline Basalto & 3500 & 2.65 & 680 \\
Regolito & 1500 & 0.0015 & 680 \\
\hline
\end{tabular}

Tabla 5.1. Parámetros del material para Basaltos y Regolitos, utilizados en el modelo del efecto Yarkovsky (Farinella et al. 1998).

lado opuesto, el valor de $\Delta T$ es pequeño y por ende la magnitud del efecto no resulta ser relevante. De acuerdo a lo sugerido por Farinella et al. (1998), esto ocurre cuando el radio de un objeto es comparable o menor a la profundidad de penetración térmica $l_{\mathrm{d}}$, la cual está dada por la expresión siguiente:

$$
l_{\mathrm{d}}=\sqrt{\frac{K}{\rho C \omega}},
$$

donde $K$ es la conductividad térmica, $C$ es el calor específico y $\omega=2 \pi / P_{\text {rot }}$ es la frecuencia de rotación. Si asumimos que $P_{\text {rot }}=5 \mathrm{~h}$, lo cual es consistente con estimaciones para asteroides de tamaños kilométricos, y se adoptan valores para los parámetros $K, \rho$ y $C$ asociados a basaltos (Tabla 5.1), $l_{\mathrm{d}}$ es $\sim 5.6 \mathrm{~cm}$. Por otra parte, si consideramos que $P_{\text {rot }}$ es una función del diámetro $D$ del objeto de la forma $P_{\text {rot }}=5 \mathrm{~h} \times(D / 1 \mathrm{~km})$, lo cual es consistente con experimentos de laboratorio sobre cuerpos del orden de $1 \mathrm{~cm}$ (Farinella et al. 1998), todos los objetos mayores a $\sim 3 \mu \mathrm{m}$ son más grandes que su correspondiente $l_{\mathrm{d}}$.

Para el efecto Yarkovsky diurno, la función oblicuidad $f_{\mathrm{d}}(\zeta)=\cos \zeta$ (Peterson 1976) y $\Delta T_{\mathrm{d}}$ puede ser calculada por la siguiente expresión:

$$
\frac{\Delta T_{\mathrm{d}}}{T}=\frac{2}{3} \frac{\Theta_{\mathrm{d}}}{1+2,03 \Theta_{\mathrm{d}}+2,04 \Theta_{\mathrm{d}}^{2}} .
$$

$\Theta_{\mathrm{d}}$ es un parámetro térmico definido por Farinella et al. (1998) y representa el cociente entre la escala de tiempo de emisión térmica y la escala de tiempo rotacional. Este parámetro está dado por

$$
\Theta_{\mathrm{d}}=\frac{\sqrt{\rho C K}}{2 \pi \epsilon \sigma T^{3}} \sqrt{\omega}
$$

Con el fin de obtener $\Theta_{\mathrm{d}}$, debemos cuantificar la conductividad térmica $K$, la densidad $\rho$, el calor específico $C$ y el período de rotación $P_{\text {rot }}$ como función del diámetro $D$. De acuerdo a lo indicado por O'Brien \& Greenberg (2005), nuestro modelo asume que $P_{\text {rot }}=6 \mathrm{hr}$ para cuerpos con un diámetro $D \geq 0.15 \mathrm{~km}$, mientras que, para objetos más pequeños, $P_{\text {rot }} \propto D$ (Farinella et al 1998). Además de esta transición concerniente al período de rotación, Pravec et al. (2002) indicaron que un diámetro $D \sim 0.15 \mathrm{~km}$ podría dividir cuerpos capaces de retener cantidades significativas de regolitos de aquellos que no. De acuerdo con esto, nuestro modelo asume que la densidad $\rho$ y la conductividad térmica $K$ adoptan los valores asociados a basaltos para cuerpos con diámetros $D<0.15 \mathrm{~km}$, mientras que para cuerpos mayores, adoptan los valores asociados a regolitos, con una suave variación entre ellas alrededor de los $0.15 \mathrm{~km}$ (O’Brien \& Greenberg 2005):

$$
\begin{aligned}
\rho & =\rho_{\mathrm{r}}+\frac{\rho_{\mathrm{b}}-\rho_{\mathrm{r}}}{2}\left(1+\tanh \left(2 \log \left(\frac{0,15 \mathrm{~km}}{D}\right)\right)\right) \\
K & =K_{\mathrm{r}}+\frac{K_{\mathrm{b}}-K_{\mathrm{r}}}{2}\left(1+\tanh \left(2 \log \left(\frac{0,15 \mathrm{~km}}{D}\right)\right)\right),
\end{aligned}
$$



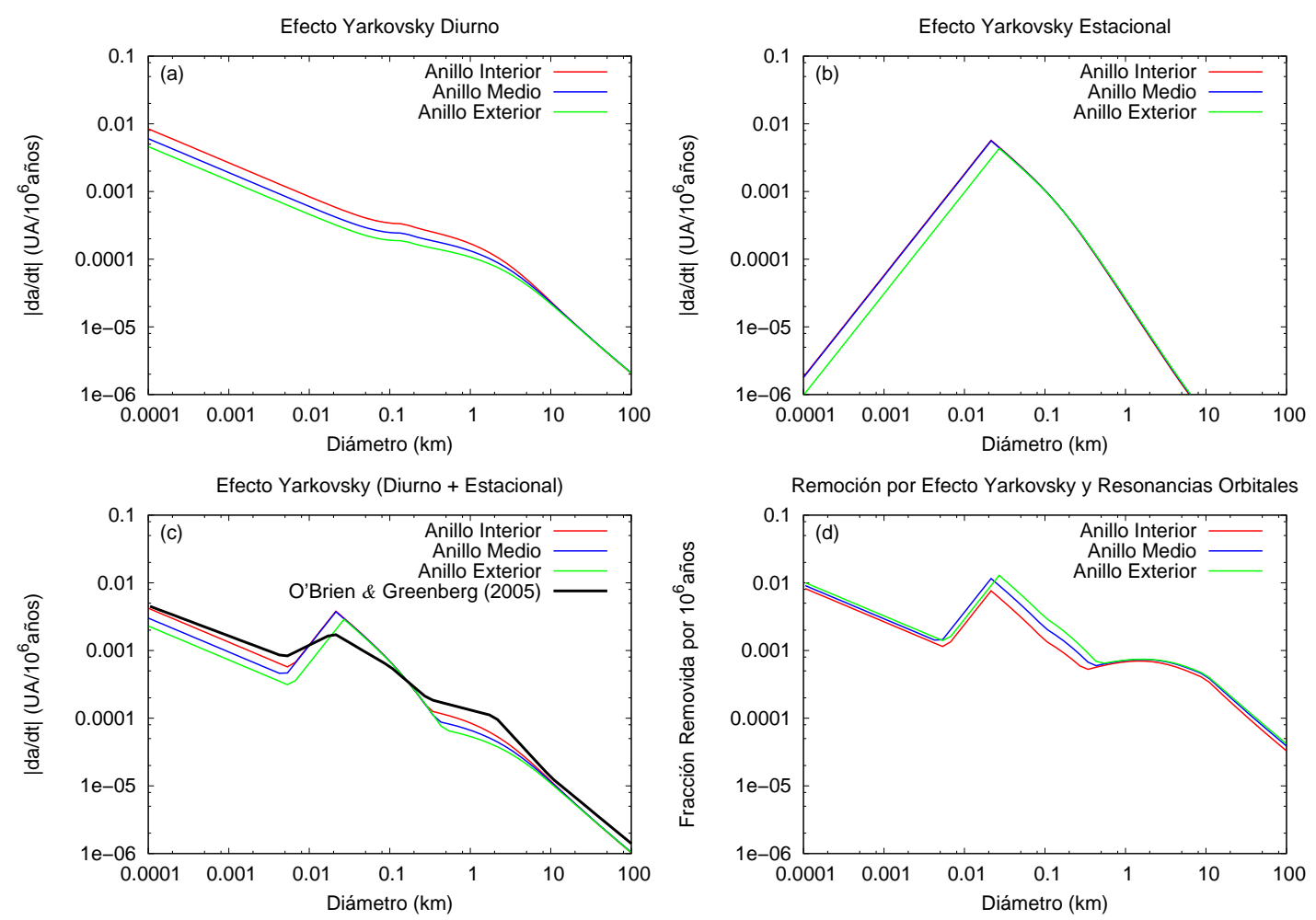

Figura 5.7. Nuestras estimaciones de la tasa de cambio del semieje mayor $\dot{a}$ debido a la variante diurna y estacional del efecto Yarkovsky son mostradas en a) y b), respectivamente. La tasa de cambio efectiva del semieje mayor debido a este efecto es ilustrada en c). Por otra parte, la tasa de remoción de asteroides debida a la acción del efecto Yarkovsky y las resonancias orbitales, es mostrada en d) como una función del diámetro. Cada gráfico expone nuestras estimaciones concernientes a los Anillos Interior, Medio y Exterior del Cinturón Principal.

donde $\rho_{\mathrm{b}}$ y $K_{\mathrm{b}}$ son los parámetros correspondientes a basaltos, mientras que $\rho_{\mathrm{r}}$ y $K_{\mathrm{r}}$ son los parámetros correspondientes a regolitos, expuestos en la Tabla 5.1. Finalmente, el calor específico $C$ siempre es igual a $680 \mathrm{~J} \mathrm{~kg}^{-1} \mathrm{~K}^{-1}$ en todas nuestras simulaciones, ya que no existen diferencias entre basaltos y regolitos concernientes a este parámetro (Tabla 5.1). Una vez que estos parámetros son especificados como función del tamaño, la tasa de cambio del semieje mayor puede ser calculada para cualquier objeto de diámetro $D$. En efecto, $\Theta_{\mathrm{d}}$ es determinado a partir de la Ecuación 5.17, y luego la diferencia de temperatura $\Delta T_{\mathrm{d}}$ es derivada a través de la Ecuación 5.16. Con esto, la fuerza por unidad de masa $F_{Y}$ generada por el efecto Yarkovsky diurno es calcula a partir de la Ecuación 5.11, y luego la tasa de cambio del semieje mayor es determinada haciendo uso de la Ecuación 5.10.

La Figura 5.7a muestra la tasa de cambio del semieje mayor debido al efecto Yarkovsky diurno como una función del diámetro para cuerpos pertenecientes a los Anillos Interior, Medio y Exterior del Cinturón Principal. En este análisis, asumimos a la oblicuidad igual a $0^{\circ}$ con el objetivo de maximizar la efectividad del efecto Yarkovsky diurno (Sección 5.4.1). 


\section{Efecto Yarkovsky Estacional}

Al igual que para la variante diurna, el efecto estacional deja de ser efectivo cuando el tamaño de un objeto resulta ser comparable o menor a la profundidad de penetración térmica $l_{\mathrm{s}}$, la cual, en este caso, está dada por la siguiente expresión:

$$
l_{\mathrm{s}}=\sqrt{\frac{K}{\rho C n}},
$$

donde $K, \rho$ y $C$ son los parámetros del material ya definidos y $n=2 \pi / P_{\text {orb }}$ es el movimiento orbital medio. Asumiendo una órbita con un semieje mayor de 2 UA y los valores para los parámetros del material asociados a basaltos, $l_{\mathrm{s}} \sim 4 \mathrm{~m}$. Los resultados de Farinella et al. (1998) indican que el pico de eficiencia del efecto estacional ocurre cuando el diámetro $D \sim$ $4 l_{\mathrm{s}}$.

La descripción matemática del efecto Yarkovsky estacional es algo más complicada que aquella ya descripta para la variante diurna. Sin embargo, para cuerpos con diámetros $D \gtrsim$ $4 l_{\mathrm{s}}$, O'Brien \& Greenberg (2005) encontraron que el efecto estacional puede ser aproximado tratándolo como un efecto diurno con frecuencia $n$ (movimiento orbital medio) en lugar de $w$ (frecuencia de rotación), y sabiendo que la asimetría estacional debe ser tenida en cuenta en sólo una fracción de la órbita. De este modo, la diferencia de temperatura efectiva $\Delta T_{\mathrm{s}}$ puede ser calculada por la expresión

$$
\frac{\Delta T_{\mathrm{s}}}{T}=\frac{2}{3} \frac{\Theta_{\mathrm{s}}}{1+2,03 \Theta_{\mathrm{s}}+2,04 \Theta_{\mathrm{s}}^{2}} \bar{f}_{\mathrm{a}},
$$

donde el parámetro térmico $\Theta_{\mathrm{s}}$ está dado por

$$
\Theta_{\mathrm{s}}=\frac{\sqrt{\rho C K}}{2 \pi \epsilon \sigma T^{3}} \sqrt{n}
$$

y el factor $\bar{f}_{\text {a }}$ tiene en cuenta la asimetría parcial. Para cuantificar este factor, consideremos un cuerpo de radio $R$ cuyo eje de rotación se ubica en el plano orbital, tal como lo muestra la Figura 5.8. Notemos que el ángulo $\phi$ marcado en dicha figura juega el rol de una latitud. Sin embargo, $\phi$ no corresponde a la latitud real del cuerpo, la cual sería medida desde el plano ecuatorial. Para un dado valor de la anomalía media $M$, la región del hemisferio sur iluminada por el Sol es aquella que va desde $\phi=0$ a $\phi=\pi-M$, mientras que la correspondiente al hemisferio norte abarca desde $\phi=\pi-M$ a $\phi=\pi$. Por otra parte, es posible definir, a modo de longitud, un ángulo $\theta$, el cual se mide sobre la dirección especificada por el Sol y el centro del cuerpo. Notemos nuevamente que $\theta$ no corresponde a la longitud real del cuerpo, la cual sería medida sobre el plano ecuatorial. A partir de esto, es posible calcular, para cada hemisferio, el área proyectada iluminada por el Sol, la cual es directamente proporcional al calor recibido por el cuerpo. Para el hemisferio sur, el área proyectada $A_{\mathrm{S}}$ iluminada por el Sol se obtiene integrando sobre el ángulo $\phi$ desde 0 a a $\pi-M$ y sobre $\theta$ desde 0 a $\pi$, obteniendo

$$
\begin{aligned}
A_{\mathrm{S}} & =\int_{0}^{\pi-M} \int_{0}^{\pi} R \mathrm{~d} \theta R \sin \theta \mathrm{d} \phi \sin \theta \sin \phi, \\
& =\frac{\pi R^{2}}{2}(1+\cos M) .
\end{aligned}
$$




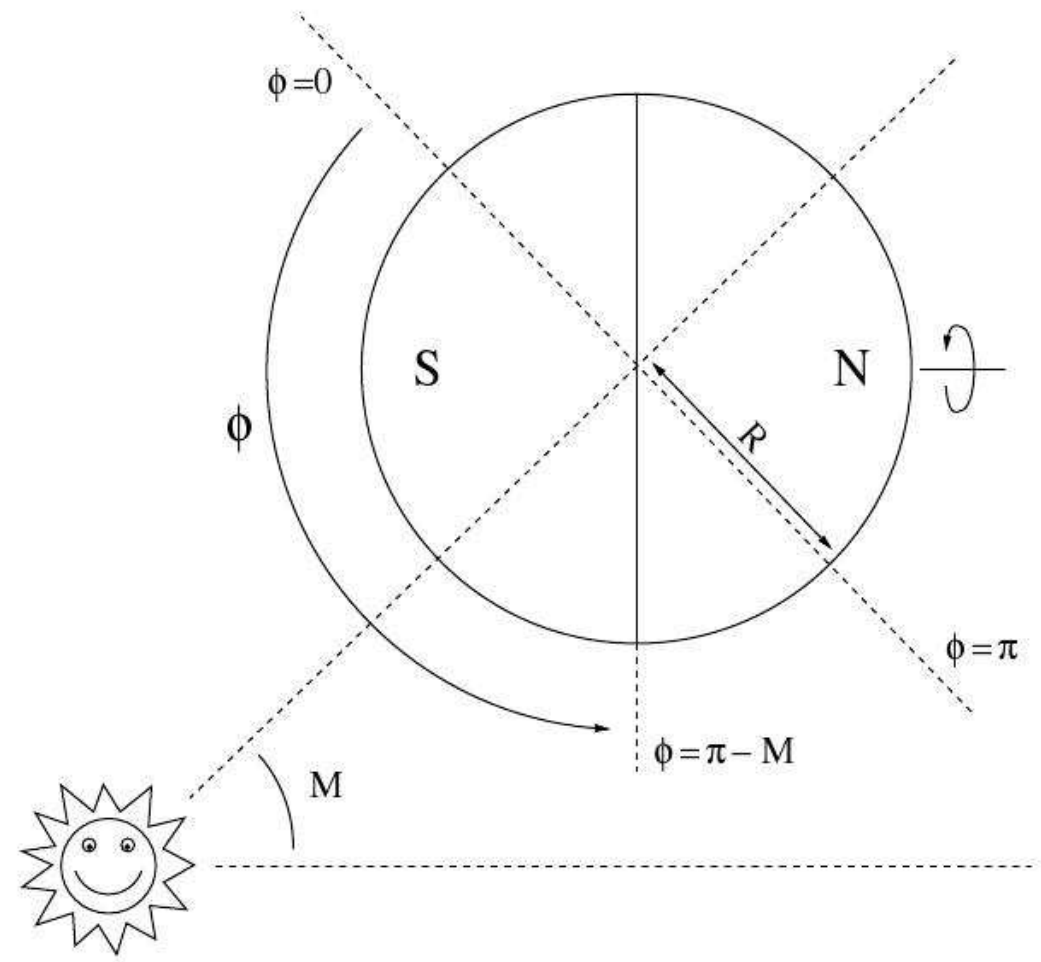

Figura 5.8. Geometría utilizada para calcular el factor de asimetría $\bar{f}_{\mathrm{a}}$ asociado a la variante estacional del efecto Yarkovsky. Para un dado valor de la anomalía media $M$, la región del hemisferio sur iluminada por el Sol es aquella que va desde $\phi=0$ a $\phi=\pi-M$, mientras que la correspondiente al hemisferio norte abarca desde $\phi=\pi-M$ a $\phi=\pi$. Figura extraída de O'Brien \& Greenberg (2005).

Por otra parte, para el hemisferio norte, el área proyectada $A_{\mathrm{N}}$ iluminada por el Sol se obtiene integrando sobre el ángulo $\phi$ desde $\pi-M$ a $\pi$ y sobre $\theta$ desde 0 a $\pi$, obteniendo

$$
\begin{aligned}
A_{\mathrm{N}} & =\int_{\pi-M}^{\pi} \int_{0}^{\pi} R \mathrm{~d} \theta R \sin \theta \mathrm{d} \phi \sin \theta \sin \phi, \\
& =\frac{\pi R^{2}}{2}(1-\cos M) .
\end{aligned}
$$

O’Brien \& Greenberg (2005) definen el factor de asimetría $f_{\mathrm{a}}$ como

$$
f_{\mathrm{a}}=\frac{A_{\mathrm{S}}-A_{\mathrm{N}}}{\pi R^{2}}
$$

con lo cual $f_{\mathrm{a}}=\cos M$. A partir de esto, el valor medio de $f_{\mathrm{a}}$ para una órbita circular está dado por

$$
\begin{aligned}
\bar{f}_{\mathrm{a}} & =\frac{2}{\pi} \int_{0}^{\pi / 2} \cos M \mathrm{~d} M, \\
& =\frac{2}{\pi} .
\end{aligned}
$$


Una vez que este parámetro es especificado, la tasa de cambio del semieje mayor como consecuencia del efecto Yarkovsky estacional puede ser calculada para cualquier objeto de diámetro $D$. En efecto, $\Theta_{\mathrm{s}}$ es determinado a partir de la Ecuación 5.21, y luego la diferencia de temperatura $\Delta T_{\mathrm{S}}$ es derivada a través de la Ecuación 5.20. Con esto, la fuerza por unidad de masa $F_{Y}$ generada por el efecto Yarkovsky estacional es calcula a partir de la Ecuación 5.11, teniendo en cuenta que en este caso la función oblicuidad $f_{\mathrm{s}}(\zeta)=-\sin ^{2} \zeta$ (Farinella et al. 1998). Finalmente, la tasa de cambio del semieje mayor es determinada haciendo uso de la Ecuación 5.10.

La Figura 5.7b muestra la tasa de cambio del semieje mayor debido al efecto Yarkovsky estacional como una función del diámetro para cuerpos pertenecientes a los Anillos Interior, Medio y Exterior del Cinturón Principal. En este análisis, asumimos a la oblicuidad igual a $90^{\circ}$ con el objetivo de maximizar la efectividad del efecto Yarkovsky estacional (Sección $5.4 .1)$.

El análisis presentado aquí para la variante estacional resulta ser preciso para cuerpos cuyos diámetros $D \gtrsim 4 l_{\mathrm{s}}$ (Ecuación 5.19). Para cuerpos menores, la descripción matemática de este efecto resulta ser más complicada. De acuerdo a lo sugerido por O'Brien \& Greenberg (2005), modelamos el efecto Yarkovsky estacional para cuerpos con diámetros $D \lesssim 4 l_{\mathrm{s}}$ asumiendo que $F_{\mathrm{Y}} \sim D^{3 / 2}$, lo cual es consistente con los cálculos de Farinella et al. (1998).

\section{Remoción de Objetos debido al Efecto Yarkovsky y las Resonancias Orbitales}

La tasa de cambio efectiva del semieje mayor $\dot{a}$ de un cuerpo debida al efecto Yarkovsky para cada uno de los tres anillos del Cinturón Principal de asteroides resulta ser una combinación de las variantes diurna y estacional. Teniendo en cuenta que los valores absolutos medios de las funciones oblicuidad $f_{\mathrm{d}}(\zeta)$ y $f_{\mathrm{s}}(\zeta)$ son $1 / 2$ y $2 / 3$, respectivamente, los valores absolutos máximos y mínimos de $\dot{a}$ están dados por

$$
\begin{aligned}
|\dot{a}(D)|_{\min }= & \operatorname{abs}\left(\left\langle\left|f_{\mathrm{s}}(\zeta)\right|\right\rangle\left|\dot{a}_{\mathrm{s}}\left(D, \zeta=90^{\circ}\right)\right|\right. \\
& \left.-\left\langle\left|f_{\mathrm{d}}(\zeta)\right|\right\rangle\left|\dot{a}_{\mathrm{d}}\left(D, \zeta=0^{\circ}\right)\right|\right) \\
|\dot{a}(D)|_{\max }= & \operatorname{abs}\left(\left\langle\left|f_{\mathrm{s}}(\zeta)\right|\right\rangle\left|\dot{a}_{\mathrm{s}}\left(D, \zeta=90^{\circ}\right)\right|\right. \\
& \left.+\left\langle\left|f_{\mathrm{d}}(\zeta)\right|\right\rangle\left|\dot{a}_{\mathrm{d}}\left(D, \zeta=0^{\circ}\right)\right|\right),
\end{aligned}
$$

y luego, el valor absoluto promedio de $\dot{a}$ puede ser calculado por la siguiente expresión

$$
\langle\dot{a}(D)\rangle=\frac{|\dot{a}(D)|_{\min }+|\dot{a}(D)| \max }{2},
$$

(O'Brien \& Greenberg 2005). La Figura 5.7c muestra la tasa de cambio efectiva del semieje mayor $\dot{a}$ como una función del diámetro para cuerpos pertenecientes a los Anillos Interior, Medio y Exterior del Cinturón Principal. Tales estimaciones resultan ser consistentes con aquella derivada por O’Brien \& Greenberg (2005) para el cinturón de asteroides completo.

Una vez que $\langle\dot{a}(D)\rangle$ ha sido determinada, la fracción de cuerpos de diámetro $D$ removidos por unidad de tiempo puede ser computada a partir de la expresión

$$
f_{\text {rem }}(D)=\frac{\langle\dot{a}(D)\rangle}{\Delta a} N_{\text {res }}
$$


donde $\Delta a$ es el ancho efectivo de la región considerada y $N_{\text {res }}$ es el número de resonancias que pueden remover cuerpos de un dado diámetro $D$ desde tal región. La fracción de cuerpos removidos por unidad de tiempo no es linealmente proporcional a $\langle\dot{a}(D)\rangle$ ya que $N_{\text {res }}$ depende del diámetro $D$. En efecto, cuerpos pequeños $(D \lesssim 0.1 \mathrm{~km})$ tienen altas tasas de cambio de sus semiejes debido al efecto Yarkovsky, por lo cual pueden saltar las resonancias débiles, siendo sólo removidos por las resonancias poderosas. De acuerdo a lo realizado por O'Brien \& Greenberg (2005), asumimos que las resonancias débiles comienzan a ser efectivas para cuerpos con diámetros $D \sim 0.1 \mathrm{~km}$, mientras que todas ellas llegan a ser completamente efectivas para objetos con diámetros $D \gtrsim 10 \mathrm{~km}$. De este modo, consideramos que $N_{\text {res }}$ es igual al número de resonancias poderosas en la región considerada para objetos con diámetros $D \leq 0.1 \mathrm{~km}$ mientras que $N_{\text {res }}$ es igual al número de resonancias poderosas más el número aproximado de resonancias difusivas para objetos con diámetros $D \geq 10 \mathrm{~km}$, asumiendo una variación lineal para diámetros intermedios. Para evaluar el número de resonancias poderosas en cada uno de los tres anillos del Cinturón Principal, tenemos en cuenta las posibles rutas de escape desde tales regiones. $\Delta a /\langle\dot{a}(D)\rangle$ representa una estimación del tiempo de vida medio de un cuerpo de diámetro $D$ en una región con un ancho efectivo $\Delta a$, donde las rutas de escape están localizadas en los bordes. Ya que los Anillos Interior y Medio del Cinturón Principal de asteroides están limitados por las resonancias poderosas $\nu_{6}, 3: 1$ y 5:2 y además, consideramos que no hay otras resonancias fuertes dentro de tales anillos, el número de resonancias poderosas será igual a 1 para tales regiones. Para el caso del Anillo Exterior, cuyos límites están dados por las resonancias poderosas 5:2 y 2:1, también consideramos la existencia de la resonancia 7:3 a $\sim 2.96$ UA. Como consecuencia de esto, el número de resonancias poderosas es igual a 2 para el Anillo Exterior del Cinturón Principal. Por otra parte, asumimos la presencia de 16, 12 y 18 resonancias difusivas en los anillos Interior, Medio y Exterior del cinturón de asteroides, respectivamente. La Figura 5.7d muestra las tasas de remoción de asteroides debido a la acción del efecto Yarkovsky y las resonancias orbitales para cada uno de los tres anillos del Cinturón Principal, obtenidas a partir de nuestras simulaciones.

Sin embargo, al igual que O'Brien \& Greenberg (2005), nuestro modelo no tiene en cuenta el efecto YORP ni las reorientaciones colisionales de los ejes de spin de los asteroides. Debido a esto, es probable que las tasas reales de remoción de asteroides desde las diferentes regiones del Cinturón Principal difieran de nuestras estimaciones, aunque ellas probablemente muestren una tendencia similar y sean del mismo orden de magnitud. De este modo, ya que las tasas de remoción obtenidas a partir de nuestro análisis resultan ser sólo una estimación, decidimos modificarlas levemente con el objetivo de obtener mejores ajustes a las poblaciones observadas del modelo La Figura 5.9 muestra las tasas de remoción de asteroides por acción del efecto Yarkovsky y las resonancias orbitales usadas en nuestro algoritmo, para cada unos de los tres anillos del Cinturón Principal.

\subsection{Parámetros Colisionales}

\subsubsection{Probabilidad Intrínseca de Colisión y Velocidad de Impacto}

Para llevar a cabo el tratamiento colisional del modelo, calculamos los valores medios de la probabilidad intrínseca de colisión $\left\langle P_{\mathrm{i}}\right\rangle$ y la velocidad de impacto $\langle V\rangle$ para colisiones entre asteroides del Cinturón Principal en cada anillo y entre anillos, así como también para 

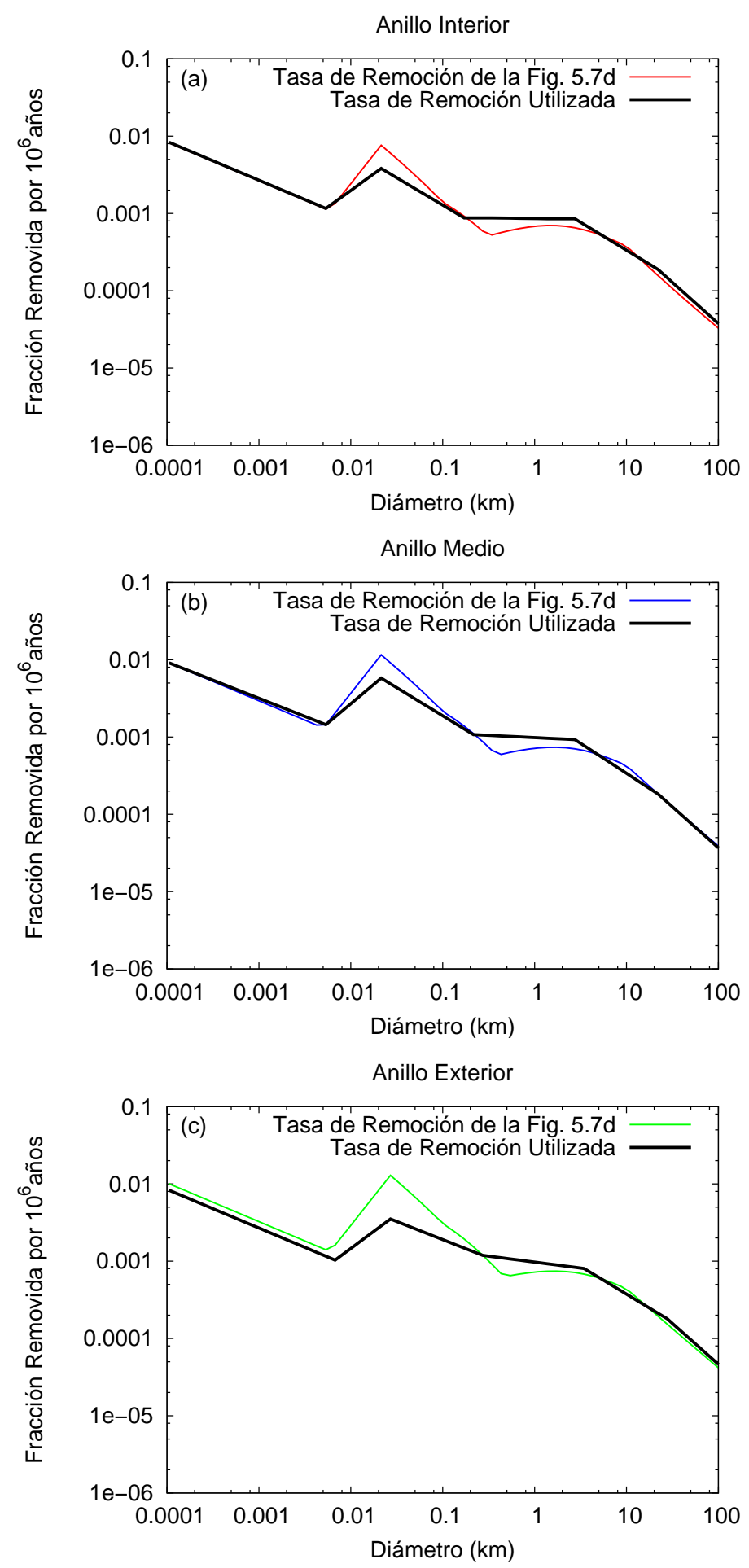

Figura 5.9. Tasas de remoción de asteroides debidas al efecto Yarkovsky y las resonancias orbitales, usadas en nuestras simulaciones para cada uno de los tres anillos del Cinturón Principal. Para comparación, se muestran las estimaciones obtenidas en nuestro análisis original e ilustradas en la Figura 5.7d. 


\begin{tabular}{|c|c|c|c|c|}
\hline Poblaciones & $\begin{array}{c}\left\langle P_{\mathrm{i}}\right\rangle \\
\left(10^{-18} \mathbf{k m}^{-2} \text { año }^{-1}\right)\end{array}$ & $\begin{array}{c}\langle V\rangle \\
\left(\mathrm{km} \mathrm{s}^{-1}\right)\end{array}$ & $f_{\text {keo }}$ & $\gamma$ \\
\hline & \multicolumn{4}{|c|}{ Cinturón Principal-Cinturón Principal } \\
\hline AI-AI & 9.8 & 3.7 & 0.05 & 0.5 \\
\hline AI-AM & 8.6 & 4.4 & 0.1 & 0.2 \\
\hline AI-AE & 1.4 & 4.4 & 0.1 & 0.3 \\
\hline AM-AM & 1.7 & 4.3 & 0.3 & 0.5 \\
\hline AM-AE & 3.5 & 4 & 0.3 & 0.2 \\
\hline \multirow[t]{2}{*}{$\mathrm{AE}-\mathrm{AE}$} & 2.74 & 3.3 & 0.35 & 0.5 \\
\hline & \multicolumn{4}{|c|}{ Cinturón Principal-NEAs } \\
\hline AI-NEA & 6.2 & 9.9 & 0.2 & 0.4 \\
\hline AM-NEA & 2.7 & 9.3 & 0.2 & 0.4 \\
\hline \multirow[t]{2}{*}{ AE-NEA } & 1.5 & 8.1 & 0.2 & 0.4 \\
\hline & \multicolumn{4}{|c|}{ NEAs-NEAs } \\
\hline NEA-NEA & 7.2 & 14.5 & 0.2 & 0.4 \\
\hline
\end{tabular}

Tabla 5.2. Parámetros Colisionales. Las primeras dos columnas exponen los valores medios de la probabilidad intrínseca de colisión $\left\langle P_{\mathrm{i}}\right\rangle$ y la velocidad de impacto $\langle V\rangle$, mientras que las dos últimas presentan los valores de $f_{\mathrm{ke}_{0}} \mathrm{y} \gamma$ para las diferentes poblaciones de nuestro modelo.

colisiones entre NEAs, y entre NEAs y objetos del Cinturón Principal en cada anillo. Para hacer esto, usamos el algoritmo numérico desarrollado por Marzari et al. (1996), basado en la integración numérica de 3000 asteroides reales, sujetos a las perturbaciones de Júpiter y Saturno. La simulación es desarrollada con el código simpléctico EVORB (Fernández et al. 2002), utilizando un paso de integración de 0.5 años. Los resultados obtenidos son mostrados en las dos primeras columnas de la Tabla 5.2.

\subsubsection{Leyes de Impacto}

De acuerdo a los argumentos propuestos en la Sección 3.3, decidimos seleccionar a $Q_{\mathrm{S}} \mathrm{y}$ $f_{\text {ke }}$ como parámetros de entrada de nuestro modelo colisional.

La ley $Q_{\mathrm{S}}$ seleccionada para nuestro estudio es mostrada en la Figura 5.10a, la cual puede ser calculada a partir de una expresión de la forma

$$
Q_{\mathrm{S}}=C_{1} D^{-\lambda_{1}}\left(1+\left(C_{2} D\right)^{\lambda_{2}}\right),
$$

donde $C_{1}, C_{2}, \lambda_{1}$, y $\lambda_{2}$ son coeficientes constantes cuyos valores son $2.85,1.8,0.695$ y 2.22 , respectivamente. Como resulta evidente a partir de la Figura 5.10a, esta ley $Q_{\mathrm{S}}$ está en un buen acuerdo con las estimaciones propuestas por diferentes autores (Farinella et al. 1982; Davis et al. 1985; Housen \& Holsapple 1990; Housen 1991; Holsapple 1994; Ryan \& Melosh 1998; Benz \& Asphaug 1999).

Por otra parte, de acuerdo a lo discutido en la Sección 4.4, el parámetro $f_{\text {ke }}$ puede ser 

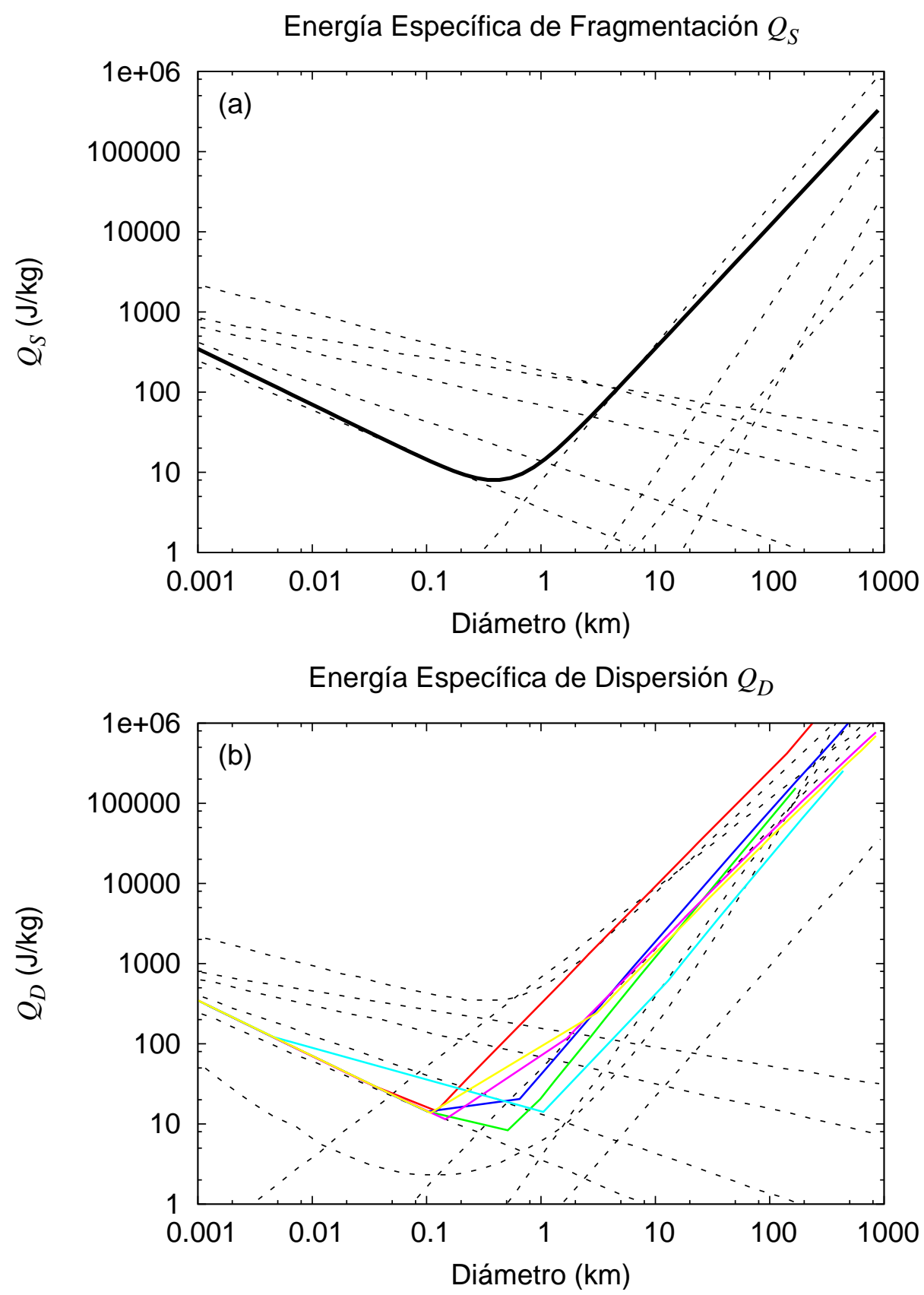

Figura 5.10. Leyes de Impacto. a) La curva sólida denota la ley $Q_{\mathrm{S}}$ usada en nuestras simulaciones. Las líneas punteadas representan las estimaciones propuestas por diferentes autores, vistas en la Sección 3.3 (Figura 3.14a). b) Las curvas de colores denotan las leyes $Q_{\mathrm{D}}$ generadas a partir de nuestra ley $Q_{\mathrm{S}}$ y las diferentes funciones $f_{\mathrm{ke}}$ asociadas a las distintas poblaciones del modelo. Las líneas punteadas representan las estimaciones propuestas por diferentes autores, vistas en la Sección 3.3 (Figura 3.14b). 
expresado como

$$
f_{\mathrm{ke}}=f_{\mathrm{ke}_{0}}\left(\frac{D}{1000 \mathrm{~km}}\right)^{\gamma} .
$$

Las últimas dos columnas de la Tabla 5.2 muestran los valores de $f_{\mathrm{ke}_{0}} \mathrm{y} \gamma$ para las diferentes poblaciones de nuestro modelo, los cuales son consistentes con aquellos discutidos por Davis et al. (1989).

La combinación de una ley $Q_{\mathrm{S}}$ y un parámetro $f_{\text {ke }}$ produce una dada $Q_{\mathrm{D}}$. Las leyes $Q_{\mathrm{D}}$ generadas a partir de nuestra ley $Q_{\mathrm{S}}$ y las diferentes funciones $f_{\mathrm{ke}}$ son mostradas en la Figura 5.10b. Tales leyes $Q_{\mathrm{D}}$ son consistentes con aquellas formuladas por diferentes autores (Farinella et al. 1982; Davis et al. 1985; Housen \& Holsapple 1990; Holsapple 1994; Love \& Ahrens 1996; Melosh \& Ryan 1997; Durda et al. 1998; Ryan \& Melosh 1998; Benz \& Asphaug 1999). Además, las mismas están en un buen acuerdo con los experimentos de impacto realizados en laboratorios, los cuales obtienen valores de $Q_{\mathrm{D}}$ cercanos a $1500 \mathrm{~J} \mathrm{~kg}^{-1}$ para blancos de $\sim 8 \mathrm{~cm}$ de diámetro.

\subsection{Modelo Numérico de Evolución Colisional y Dinámica}

En esta sección presentamos el modelo dinámico y colisional usado para estudiar la evolución simultánea del Cinturón Principal de asteroides y la población de NEAs.

De acuerdo a lo sugerido por Campo Bagatin et al. (1994a) y Campo Bagatin (1998), un sistema colisional cuya población tiene una distribución de masa truncada a un dado valor $m$, lleva a ondas en la distribución de tamaños de los cuerpos. Con el objetivo de eliminar este efecto, nuestro algoritmo no evoluciona en el tiempo los primeros 60 bines de tamaño, cuyos diámetros centrales van desde $10^{-10}$ a $10^{-4} \mathrm{~km}$. Para los NEAs y cada uno de los tres anillos del cinturón de asteroides, esta parte de la población es usada únicamente a modo de proyectiles para computar tasas de impacto con cuerpos más grandes, y su distribución de tamaños es determinada en cada paso de tiempo, extrapolando la pendiente de la distribución asociada a los 10 bines de tamaño siguientes.

La descripción matemática de nuestro algoritmo incluye procesos colisionales y mecanismos dinámicos con el objetivo de determinar el destino final de los fragmentos generados en una colisión.

Supongamos que un objeto $o$ de la población $p$ colisiona con otro objeto $o^{\prime}$ perteneciente a la población $p^{\prime}$. $p$ y $p^{\prime}$ representan a cualquiera de las cuatro poblaciones de nuestro código. Haciendo uso del modelo colisional descripto en el Capítulo 4, es posible derivar las distribuciones de fragmentos colisionales resultantes de los cuerpos $o$ y $o^{\prime}$. Tales distribuciones son guardadas en los vectores $F_{o}^{p}(k)$ y $F_{o^{\prime}}^{p^{\prime}}(k)$, donde $k$ representa un bin de tamaño tal como fue definido en la Sección 5.2. Nuestro modelo colisional también nos permite determinar las distribuciones de velocidades de los fragmentos colisionales eyectados desde los cuerpos $o$ y $o^{\prime}$, las cuales son referidas como $V_{o}^{p}(k)$ y $V_{o^{\prime}}^{p^{\prime}}(k)$, respectivamente. Por otro lado, este modelo nos conduce a un cálculo sencillo de la velocidad de escape del sistema de los dos cuerpos colisionantes, la cual es simbolizada por $V_{\text {esc }}$.

Una vez hecho esto, nuestro objetivo principal es determinar el destino de los fragmentos, especificando la población en la cual terminan residiendo. Si los fragmentos asociados a un dado bin de tamaño $k$ de los vectores $F_{o}^{p}$ y $F_{o^{\prime}}^{p^{\prime}}$ tienen una velocidad menor a la velocidad 
de escape $V_{\text {esc }}$, ellos son reacumulados y su masa es sumada entre si. Luego, esta masa es adicionada a aquellas correspondientes al fragmento remanente más grande o al cuerpo craterizado final que resultan de los cuerpos o y $o^{\prime}$, dependiendo si cada uno de estos sufrió una colisión catastrófica o una craterización. Este procedimiento nos permite obtener la masa de un objeto reacumulado único y a partir de la misma el bin de tamaño $k_{\mathrm{r}}$ al cual pertenece. La población final de residencia de este objeto reacumulado es aquella correspondiente al objeto colisionante de mayor tamaño. Si ambos cuerpos que impactan son de igual tamaño, la población final de residencia del objeto reacumulado es seleccionada al azar entre las poblaciones asociadas a los dos objetos que participan de la colisión.

Los fragmentos distribuidos en $F_{o}^{p}(k)$ y $F_{o^{\prime}}^{p^{\prime}}(k)$ que tienen velocidades mayores a $V_{\text {esc }}$ escapan del campo gravitacional del sistema que constituyen los dos cuerpos colisionantes. Para determinar sus destinos finales, proponemos un procedimiento simple y eficaz. En cada intervalo de tiempo, una única órbita característica es generada al azar para cada una de las poblaciones del modelo, la cual es usada independientemente del tamaño de los cuerpos involucrados en la colisión. Para los anillos del cinturón de asteroides, asignamos excentricidades $e$ entre 0 y 0.3 , inclinaciones $i$ entre 0 y $20^{\circ}$ y semiejes mayores $a$ en una forma tal que $2 \leq$ $a \leq 2.5$ UA para el Anillo Interior, $2.5 \leq a \leq 2.82$ UA para el Anillo Medio y $2.82 \leq a \leq$ 3.27 UA para el Anillo Exterior. En cada caso, la combinación de valores $(a, i)$ debe estar por debajo de la localización de la resonancia secular $\nu_{6}$, mientras que la combinación de valores $(a, e)$ debe caer afuera de los gaps asociados con las resonancias 3:1 y 5:2, cuyos límites fueron ya discutidos en la Sección 5.3. Para la población de NEAs, usamos parámetros orbitales $0 \leq$ $a \leq 3.4 \mathrm{UA}, 0 \leq e \leq 0.7$ y $0 \leq i \leq 40^{\circ}$, los cuales son combinados en una forma tal que las distancias perihélicas $q$ y las distancias afélicas $Q$ son siempre menores a 1.3 UA y mayores a 0.983 UA, respectivamente, de acuerdo a la definición de un NEA. Finalmente, dada la longitud del nodo ascendente $\Omega$, el argumento del perihelio $\omega$ y la anomalía media $M$ entre 0 y $360^{\circ}$, una órbita puede ser asignada y a partir de esto derivar un par posición-velocidad $\left(\overrightarrow{X^{p}}, \overrightarrow{V^{p}}\right)$ para todos los cuerpos de cada población, simbolizada por el índice $p$. En la Sección 5.7 , discutimos algunos aspectos relacionados a este tratamiento.

Para determinar el destino final de los fragmentos que escapan, es necesario calcular cuáles son sus elementos orbitales una vez que ellos son eyectados con una dada velocidad respecto del cuerpo que los originó. Inmediatamente antes de la colisión, asumimos que la posición y velocidad baricéntricas de los fragmentos son iguales a aquellas asociadas a su cuerpo precursor, esto es, a $\overrightarrow{X^{l}}$ y $\overrightarrow{V^{l}}$, respectivamente. Inmediatamente después de la colisión, consideramos que la posición baricéntrica de los fragmentos es equivalente a aquella asociada a su cuerpo precursor, mientras que la velocidad relativa de los mismos con respecto a este último, $V_{i}^{l}(k)$, es igualmente particionada entre las tres componentes cartesianas. De este modo, una simple suma entre cada una de las componentes cartesianas de $\overrightarrow{V^{l}}$ y $V_{i}^{l}(k) / \sqrt{3}$ nos permite obtener el vector baricéntrico de la velocidad inmediatamente después de la colisión. Una vez que obtenemos la posición y velocidad baricéntricas de los fragmentos posteriores al impacto, es posible calcular sus elementos orbitales y sus destinos finales. Para hacer esto, usamos los siguientes criterios:

1. Los fragmentos finalizan en la población de los NEAs, si se satisface cualquiera de las siguientes condiciones:

- la distancia afélica $Q \geq 0,983$ UA y la distancia perihélica $q \leq 1,3 \mathrm{UA}$, 
- el semieje mayor $a<2$ UA,

- la combinación de valores $(a, i)$ cae por encima de la localización de la resonancia secular $\nu_{6}$,

- la combinación de valores $(a, e)$ cae dentro del gap asociado con la resonancia de movimientos medios 3:1,

- la combinación de valores $(a, e)$ cae dentro del gap asociado con la resonancia de movimientos medios 5:2.

2. Los fragmentos finalizan en el Anillo Interior del Cinturón Principal si

- $2 \leq a \leq 2.5$ UA y las combinaciones de los valores $(a, e)$ y $(a, i)$ caen fuera del gap asociado con la resonancia 3:1 y debajo de la localización de la resonancia secular $\nu_{6}$, respectivamente.

3. Los fragmentos finalizan en el Anillo Medio del Cinturón Principal si

- $2.5 \leq a \leq 2.82$ UA y las combinaciones de los valores $(a, e)$ y $(a, i)$ caen fuera de los gaps asociados con las resonancias 3:1 y 5:2, y debajo de la localización de la resonancia secular $\nu_{6}$, respectivamente.

4. Los fragmentos finalizan en el Anillo Exterior del Cinturón Principal si

- $2.82 \leq a \leq 3.27$ UA y las combinaciones de los valores $(a, e)$ y $(a, i)$ caen fuera del gap asociado con la resonancia 5:2 y debajo de la localización de la resonancia secular $\nu_{6}$, respectivamente.

5. Finalmente, asumimos que los fragmentos son eyectados del Sistema Solar sobre órbitas hiperbólicas, dejando de participar de la evolución colisional, si la excentricidad $e \geq 1$ o $a>3.27 \mathrm{UA}$.

La información de este procedimiento puede ser guardada en dos matrices $F_{o}^{p}\left(k, p^{\prime \prime}\right)$ y $F_{o^{\prime}}^{p^{\prime}}\left(k, p^{\prime \prime \prime}\right)$, en las cuales los índices $p^{\prime \prime}$ y $p^{\prime \prime \prime}$ indican las poblaciones en las cuales terminan residiendo los fragmentos del bin de tamaño $k$ eyectados desde los cuerpos $o$ y $o^{\prime}$, respectivamente.

La frecuencia de colisión entre el cuerpo o de diámetro $D_{o}$ perteneciente a la población $p$ y el cuerpo $o^{\prime}$ de diámetro $D_{o^{\prime}}$ perteneciente a la población $p^{\prime}$ puede ser calculada a partir de la siguiente expresión

$$
f_{o, o^{\prime}}^{p, p^{\prime}}=\frac{\left\langle P_{\mathrm{i}}\right\rangle^{p, p^{\prime}}}{4}\left(D_{o}+D_{o^{\prime}}\right)^{2}
$$

donde $\left\langle P_{\mathrm{i}}\right\rangle^{p, p^{\prime}}$ es el valor medio de la probabilidad intrínseca de colisión entre las poblaciones $p$ y $p^{\prime}$, y tiene unidades de $\mathrm{km}^{-2}$ año ${ }^{-1}$. Si $\Delta t$ representa un dado intervalo y $N_{o, o^{\prime}}^{p, p^{\prime}}$ es el número de pares de objetos $o$ y $o^{\prime}$ en las poblaciones $p$ y $p^{\prime}$, respectivamente, es posible obtener el número total de colisiones entre dichos objetos durante $\Delta t$ a partir de la expresión

$$
N_{\mathrm{col}}=f_{o, o^{\prime}}^{p, p^{\prime}} N_{o, o^{\prime}}^{p, p^{\prime}} \Delta t
$$

Sean $N_{o}^{p}$ el número de cuerpos o de diámetro $D_{o}$ pertenecientes a la población $p$, y $N_{o^{\prime}}^{p^{\prime}}$ el número de cuerpos $o^{\prime}$ de diámetro $D_{o^{\prime}}$ pertenecientes a la población $p^{\prime}$. Si los cuerpos colisionantes pertenecen al mismo bin de tamaño y además están en la misma población, esto 
es, si $o=o^{\prime}$ y $p=p^{\prime}$, entonces $N_{o}^{p}=N_{o^{\prime}}^{p^{\prime}}$ y por ende, el número de pares de objetos $o$ y $o^{\prime}$ en las poblaciones $p$ y $p^{\prime}$ está dado por

$$
N_{o, o^{\prime}}^{p, p^{\prime}}=\frac{N_{o}^{p}\left(N_{o^{\prime}}^{p^{\prime}}-1\right)}{2}
$$

Para todos los otros casos,

$$
N_{o, o^{\prime}}^{p, p^{\prime}}=N_{o}^{p} N_{o^{\prime}}^{p^{\prime}}
$$

A partir de esto, el número total de fragmentos asociados al bin de tamaño $k$ que finalizan en la población $p^{\prime \prime}$ luego de ser eyectados de los cuerpos $o$ de la población $p$ como resultado de colisiones con cuerpos $o^{\prime}$ de la población $p^{\prime}$, durante un intervalo $\Delta t$, está dado por $F_{o}^{p}\left(k, p^{\prime \prime}\right) f_{o, o^{\prime}}^{p, p^{\prime}} N_{o, o^{\prime}}^{p, p^{\prime}} \Delta t$. Equivalentemente, el número total de fragmentos asociados al bin de tamaño $k$ que finalizan en la población $p^{\prime \prime \prime}$ luego de ser eyectados de los cuerpos $o^{\prime}$ de la población $p^{\prime}$ como resultado de colisiones con cuerpos $o$ de la población $p$, durante un intervalo $\Delta t$, está dado por $F_{o^{\prime}}^{p^{\prime}}\left(k, p^{\prime \prime \prime}\right) f_{o, o^{\prime}}^{p, p^{\prime}} N_{o, o^{\prime}}^{p, p^{\prime}} \Delta t$. Si tenemos en cuenta las interacciones entre todos los cuerpos $o$ y $o^{\prime}$ de las cuatro poblaciones del modelo, es posible derivar la variación en el número de cuerpos asociados a un dado bin de tamaño $k$ para cada población, en cada paso de tiempo. Dichas variaciones son referidas como $\Delta N_{k}^{\mathrm{AI}}, \Delta N_{k}^{\mathrm{AM}}, \Delta N_{k}^{\mathrm{AE}}$ y $\Delta N_{k}^{\mathrm{NEA}}, \mathrm{y}$ corresponden a los Anillos Interior, Medio y Exterior del Cinturón Principal de asteroides y la población de NEAs, respectivamente.

Una vez que este tratamiento ha finalizado, la tasa de remoción de objetos por acción del efecto Yarkovsky y las resonancias orbitales debe ser incluida en nuestro análisis. De acuerdo a los resultados numéricos derivados por Bottke et al. (2002) (Sección 3.1.2), nuestro algoritmo asume que los cuerpos removidos desde los Anillos Interior, Medio y Exterior del Cinturón Principal son llevados a la población de NEAs. Por otra parte, la tasa de remoción dinámica de NEAs también debe ser tenida en cuenta en nuestro algoritmo. La Tabla 3.1 de la Sección 3.1.2 ofrece los principales resultados dinámicos derivados por Bottke et al. (2002) para objetos provenientes desde diferentes regiones fuentes de NEAs. Las regiones bajo estudio son la resonancia secular $\nu_{6}$, la resonancia de movimientos medios 3:1 con Júpiter, la población principal de Cruzadores de Marte (IMC), el Cinturón Principal exterior y la población de Cometas de la Familia de Júpiter. En la tabla mencionada se muestra, para cada una de estas regiones, el número de NEAs en estado estacionario con una magnitud $H$ menor a 18 (lo cual equivale $\mathrm{a} \sim 1 \mathrm{~km}$ de diámetro) $\mathrm{N}_{\mathrm{NEA}}$, el número de cuerpos inyectados dentro de la región de los NEAs por millón de años $\tau$, y el tiempo de vida dinámico medio de los objetos en esta población $\mathrm{T}_{\mathrm{NEA}}$. En estado estacionario, la tasa de inyección de cuerpos $\tau$ desde una dada región fuente dentro de la población de NEAs es equivalente a la tasa de remoción dinámica $\tau_{\text {rem }}$ de aquellos cuerpos desde tal población. De este modo, para una dada fuente

$$
\begin{aligned}
\tau_{\mathrm{rem}} & =\tau \\
& =\frac{\mathrm{N}_{\mathrm{NEA}}}{\mathrm{T}_{\mathrm{NEA}}} .
\end{aligned}
$$

A partir de esto, el tiempo de vida dinámico medio de todos los cuerpos en la población de NEAs provenientes desde las diferentes regiones fuentes estudiadas por Bottke et al. (2002) está dado por

$$
\left\langle\mathrm{T}_{\mathrm{NEA}}\right\rangle=\frac{\sum \mathrm{N}_{\mathrm{NEA}}}{\sum \tau}
$$


donde la suma se extiende sobre todas las fuentes mostradas en la Tabla 3.1 de la Sección 3.1.2, excepto la población de Cometas de la Familia de Júpiter. Usando los valores dados allí, obtenemos un $\left\langle\mathrm{T}_{\mathrm{NEA}}\right\rangle=1.14 \times 10^{6}$ años. Sin embargo, las simulaciones numéricas desarrolladas por Migliorini et al. (1998) sugieren que los NEAs de tamaños kilométricos podrían tener como fuente primaria a la población principal de Cruzadores de Marte (IMC). De este modo, nuestro modelo trata la remoción de objetos desde la población de NEAs usando un tiempo de vida dinámico $\left\langle\mathrm{T}_{\mathrm{NEA}}^{\mathrm{IMC}}\right\rangle=3.75 \times 10^{6}$ años (asociado con la población de Cruzadores de Marte) para cuerpos con una magnitud $H$ menor a 12 (esto es, diámetros mayores a $\sim 15 \mathrm{~km}$ ), mientras que $\left\langle\mathrm{T}_{\mathrm{NEA}}\right\rangle$ es usado para objetos con magnitudes $H$ mayores (o, equivalentemente, diámetros menores).

Con lo dicho hasta aquí, la variación total en el número de cuerpos asociados a un dado bin de tamaño $k$ para cada una de las poblaciones del modelo, en un intervalo $\Delta t$ y teniendo en cuenta procesos de remoción colisionales y dinámicos, está dada por

$$
\begin{aligned}
\left(\Delta N_{k}^{\mathrm{AI}}\right)_{\text {Total }} & =\left(\Delta N_{k}^{\mathrm{AI}}-f_{\mathrm{rem}, k}^{\mathrm{AI}} N_{k}^{\mathrm{AI}}\right) \Delta t \\
\left(\Delta N_{k}^{\mathrm{AM}}\right)_{\text {Total }} & =\left(\Delta N_{k}^{\mathrm{AM}}-f_{\mathrm{rem}, k}^{\mathrm{AM}} N_{k}^{\mathrm{AM}}\right) \Delta t \\
\left(\Delta N_{k}^{\mathrm{AE}}\right)_{\text {Total }} & =\left(\Delta N_{k}^{\mathrm{AE}}-f_{\mathrm{rem}, k}^{\mathrm{AE}} N_{k}^{\mathrm{AE}}\right) \Delta t \\
\left(\Delta N_{k}^{\mathrm{NEA}}\right)_{\text {Total }} & =\left(\Delta N_{k}^{\mathrm{NEA}}+f_{\mathrm{rem}, k}^{\mathrm{AI}} N_{k}^{\mathrm{AI}}+f_{\mathrm{rem}, k}^{\mathrm{AM}} N_{k}^{\mathrm{AM}}+f_{\mathrm{rem}, k}^{\mathrm{AE}} N_{k}^{\mathrm{AE}}-\frac{N_{k}^{\mathrm{NEA}}}{\langle\mathrm{T}\rangle}\right) \Delta t
\end{aligned}
$$

donde $f_{\text {rem }, k}^{\mathrm{AI}}, f_{\mathrm{rem}, k}^{\mathrm{AM}}$ y $f_{\mathrm{rem}, k}^{\mathrm{AE}}$ representan las tasas de remoción de objetos por acción del efecto Yarkovsky y las resonancias orbitales, asociadas a los Anillos Interior, Medio y Exterior del Cinturón Principal de asteroides, respectivamente (Sección 5.4.2). Además, según lo indicado en el último párrafo, $\langle\mathrm{T}\rangle=\left\langle\mathrm{T}_{\mathrm{NEA}}^{\mathrm{IMC}}\right\rangle$ para NEAs con diámetros $D>15 \mathrm{~km} \mathrm{y}\langle\mathrm{T}\rangle=\left\langle\mathrm{T}_{\mathrm{NEA}}\right\rangle$ para NEAs más pequeños.

Para estudiar la evolución en el tiempo de las cuatro poblaciones del modelo, el paso de tiempo $\Delta t$ es calculado en una forma tal que el cambio relativo en el número de objetos en cualquier bin de tamaño sea siempre menor que el $1 \%$.

\subsection{Resultados}

Aquí, nosotros discutimos los resultados generales obtenidos a partir de una serie de simulaciones numéricas destinadas a estudiar la evolución colisional y dinámica de los asteroides del Cinturón Principal y NEAs sobre $4.5 \times 10^{9}$ años.

\subsubsection{Distribuciones de Tamaños del Cinturón Principal de Asteroides y NEAs}

La población de asteroides del Cinturón Principal resulta ser observacionalmente completa hasta un diámetro de $\sim 30 \mathrm{~km}$. Desde hace algunos años atrás, diversos estudios observacionales tales como Spacewatch (Jedicke \& Metcalfe 1998), Sloan Digital Sky Survey (SDSS) (Ivezić et al. 2001) y Subaru Sub-km Main Belt Asteroid Survey (SMBAS) (Yoshida et al. 2003) han permitido extender nuestro conocimiento de la distribución de tamaños del Cinturón Principal hasta un diámetro de $\sim 500 \mathrm{~m}$. Como puede apreciarse de la Figura 5.11a, la 

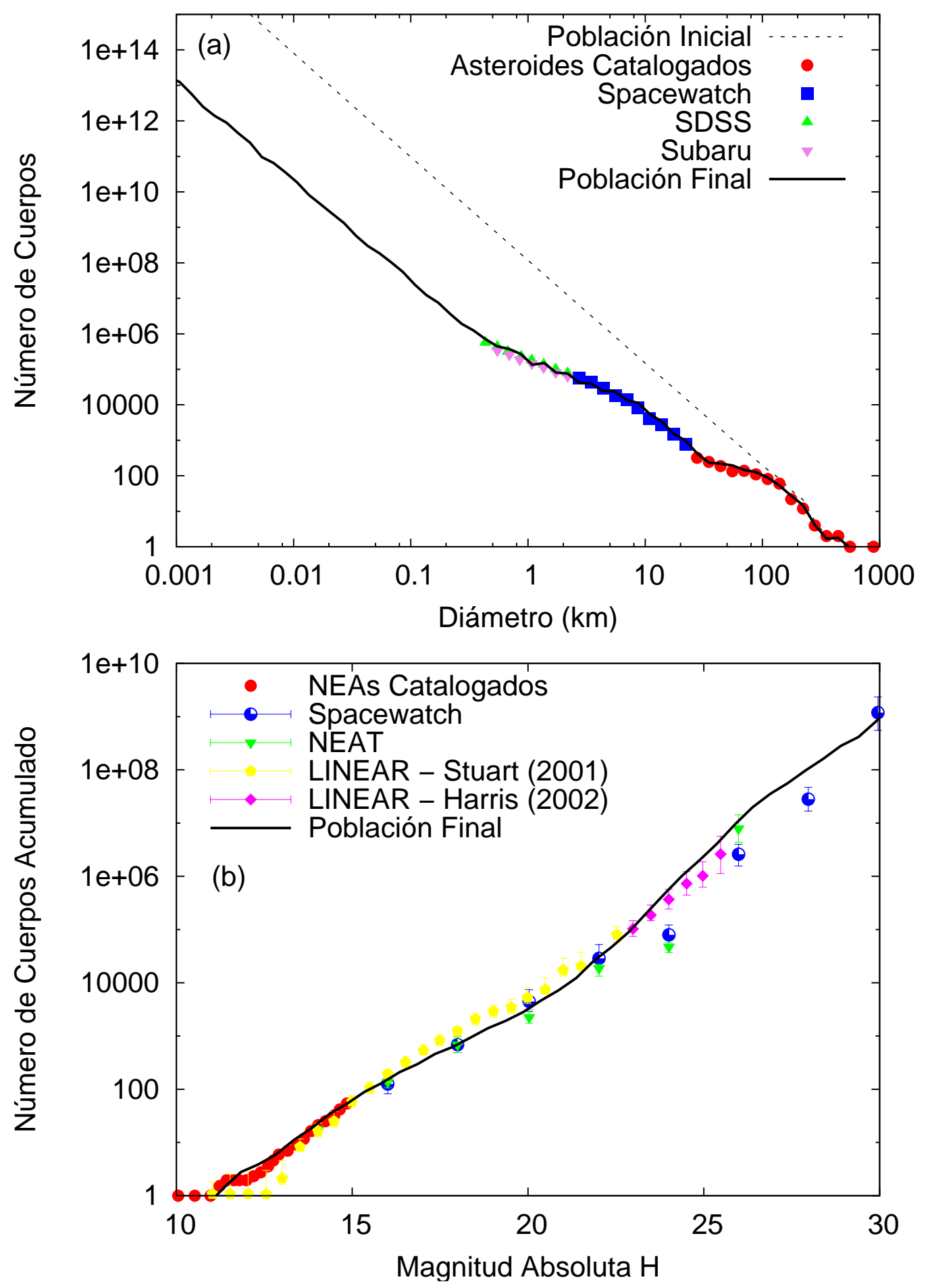

Figura 5.11. Distribución de tamaños del Cinturón Principal de asteroides a), y distribución de magnitudes $H$ de la población de NEAs b), después de $4.5 \times 10^{9}$ años de evolución. Nuestras estimaciones muestran un excelente acuerdo con los datos observacionales. 


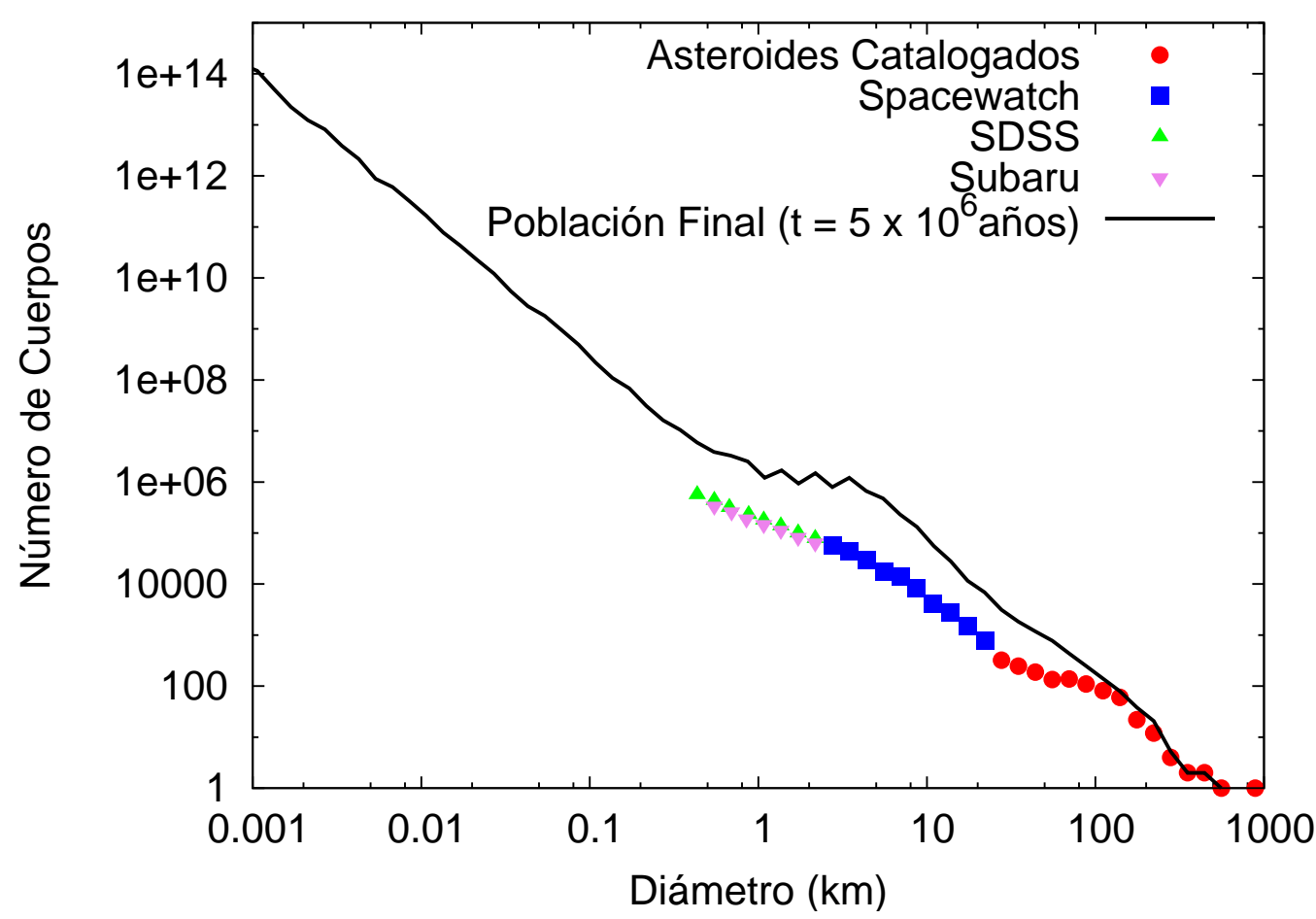

Figura 5.12. Distribución de tamaños del Cinturón Principal de asteroides después de $5 \times$ $10^{6}$ años. Estos resultados indican que la población del Cinturón Principal es predominantemente un fósil, adquiriendo su estructura de onda característica durante las primeras etapas de la evolución, lo cual es consistente con aquello discutido por Bottke et al. (2005a).

distribución de tamaños del cinturón de asteroides obtenida a partir de nuestras simulaciones es absolutamente consistente con los datos observacionales.

La Figura 5.11b muestra nuestra estimación de la distribución de tamaños de la población de NEAs, la cual es descripta en términos de la magnitud absoluta $H$. De acuerdo a lo establecido por Bowell et al. (1989), es posible derivar el diámetro $D$ de un cuerpo con una magnitud absoluta $H$ a partir de la siguiente expresión

$$
D=\frac{1347}{\sqrt{A}} 10^{-H / 5}
$$

donde $A$ es el albedo visual geométrico, al cual asumimos con un valor de 0.11 (Sección 3.2.1). Con este valor de $A$, una magnitud absoluta $H=18$ corresponde a un diámetro $D \sim 1 \mathrm{~km}$. La población de NEAs resulta ser observacionalmente completa hasta una magnitud absoluta $H=15$, lo cual corresponde a un diámetro $D \sim 4 \mathrm{~km}$. Diversos estudios observacionales han sido desarrollados con el objetivo de extender la distribución de magnitudes de la población de NEAs hasta valores mayores de $H$. En efecto, Rabinowitz et al. (2000) usaron datos del Spacewatch y del programa NEAT (Near Earth Asteroid Tracking) para derivar la población de NEAs hasta una magnitud $H \sim 30$, mientras que Stuart (2001) y Harris (2002) usaron datos del LINEAR Survey con el objetivo de extender la distribución de magnitudes de los NEAs hasta valores de $H \sim 22.5$ y 25.5, respectivamente. A partir de la Figura 5.11b, es posible observar que la población de NEAs que resulta de nuestras simulaciones es absolutamente consistente con los datos observacionales. Un resultado importante derivado a partir 
de nuestro análisis es que la distribución de magnitudes de los NEAs está determinada principalmente por la remoción dinámica de los asteroides desde el Cinturón Principal, debido a la acción del efecto Yarkovsky y las resonancias orbitales. En efecto, nuestro análisis indica que los procesos colisionales no juegan un rol relevante en la determinación cuantitativa de la población de NEAs.

Como ya hemos dicho en la Sección 5.2 y de acuerdo a lo sugerido por O’Brien \& Greenberg (2005), nosotros incluimos un breve período de evolución primordial en el comienzo de la simulación con el objetivo de reproducir los resultados de Petit et al. (1999) y Petit et al. (2001) concernientes a la remoción de masa desde un cinturón de asteroides primitivo cientos de veces más masivo que el actual. En esta fase, las poblaciones iniciales asociadas con cada uno de los tres anillos del Cinturón Principal de asteroides son multiplicadas por un factor 55 y su evolución seguida por $5 \times 10^{6}$ años. Durante este período, la intensa actividad colisional remueve $\sim 74 \%$ de la masa inicial del Cinturón Principal, llevando a una población residual para el cinturón de asteroides completo de $\sim 100$ veces su valor actual. Luego, las poblaciones iniciales residuales asociadas con cada uno de los tres anillos del Cinturón Principal son reducidas por aquel factor 55, lo cual simula la remoción de $\sim 98 \%$ de las masas de cada una de ellas. Finalmente, la evolución de la población inicial de un Cinturón Principal con una masa de $\sim 1.8$ veces su valor actual es analizada por el resto de los $4.5 \times 10^{9}$ años. Durante este tiempo, los mecanismos colisionales y dinámicos remueven $\sim 25$ y $17 \%$ de la masa inicial del Cinturón Principal, respectivamente, llevando a una población final para el cinturón de asteroides completo con una masa aproximadamente igual a su valor actual.

Por otra parte, nuestras simulaciones sugieren que la población del Cinturón Principal de asteroides adquiere una suave estructura de onda durante los primeros $5 \times 10^{6}$ años de evolución (Figura 5.12), similar a aquella observada en el cinturón de asteroides actual. Estos resultados son consistentes con aquellos obtenidos por Bottke et al. (2005a), quienes indicaron que la distribución de tamaños del Cinturón Principal es predominantemente un fósil.

\subsubsection{Remoción de Asteroides desde el Cinturón Principal}

Morbidelli \& Nesvorný (1999) y Bottke et al. (2002) realizaron estudios concernientes a la tasa de remoción dinámica de cuerpos de tamaño kilométrico desde el Cinturón Principal de asteroides. En efecto, mientras que Morbidelli \& Nesvorný (1999) estimaron el escape de $\sim 4$ cuerpos con diámetros $D \geq 5 \mathrm{~km}$ por millón de años desde el Cinturón Interior, Bottke et al. (2002) indicaron que $790 \pm 200$ cuerpos con diámetros $D \geq 1 \mathrm{~km}$ son removidos por millón de años desde el cinturón de asteroides completo.

Nuestras simulaciones indican que 1070 asteroides con diámetros $D \geq 1 \mathrm{~km}$ son removidos por millón de años desde el Cinturón Principal completo, lo cual es consistente con los análisis desarrollados por Bottke et al. (2002). Por otra parte, nuestro estudio determina el escape de 3 asteroides con diámetros $D \geq 5 \mathrm{~km}$ por millón de años desde el Anillo Interior del Cinturón Principal, sobre los últimos $3 \times 10^{9}$ años de evolución, lo cual está de acuerdo con el trabajo desarrollado por Morbidelli \& Nesvorný (1999).

La Figura 5.13 muestra las tasas de remoción de asteroides de tamaños kilométricos desde el Cinturón Principal completo teniendo en cuenta la acción del efecto Yarkovsky, la inyección colisional de material dentro de las resonancias $\nu_{6}, 3: 1$ y 5:2, y la eyección colisional más allá de las 3.27 UA. A partir de la misma podemos inferir que el efecto Yarkovsky es el proceso 


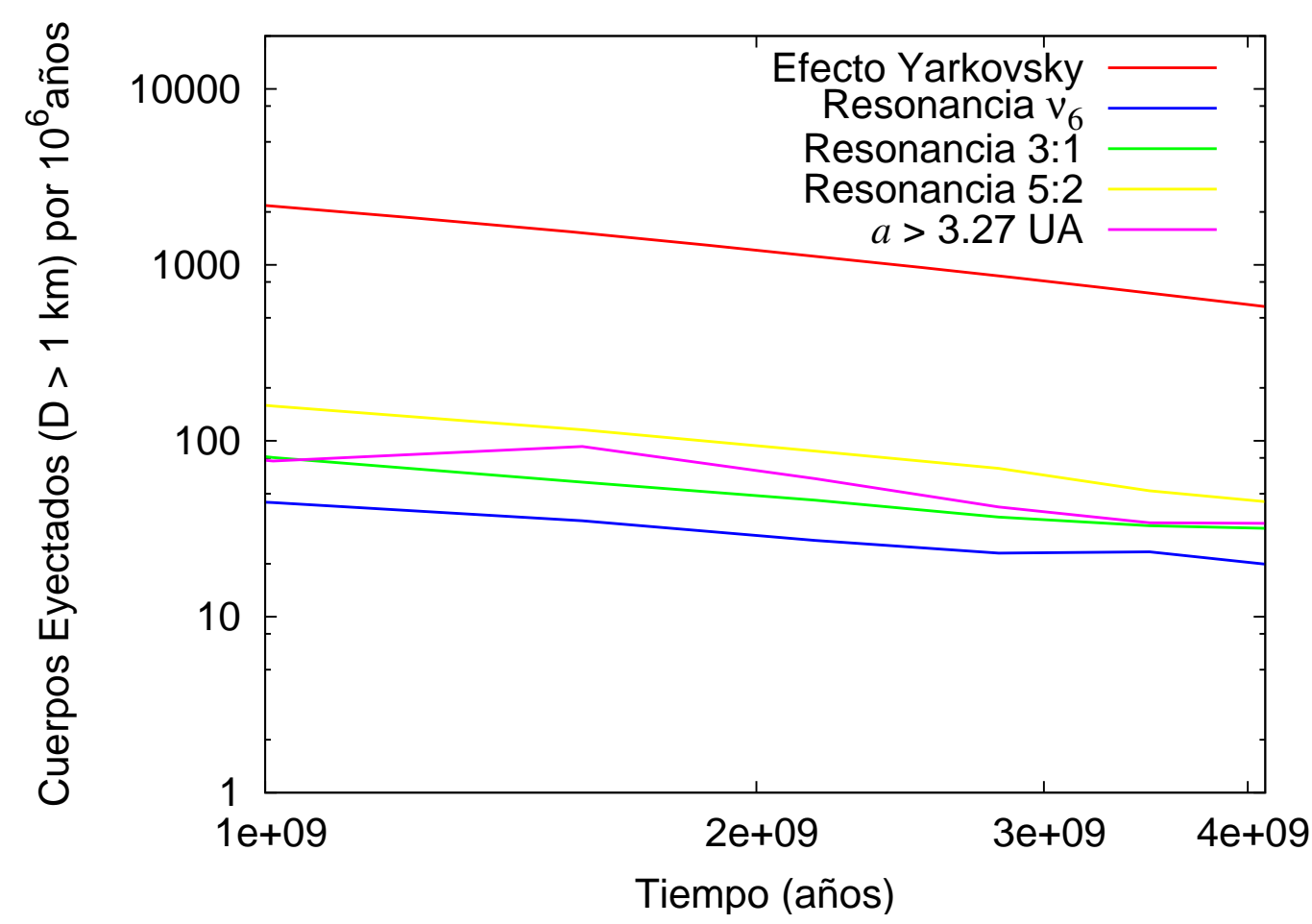

Figura 5.13. Número de cuerpos con diámetros $D \geq 1 \mathrm{~km}$ removidos por unidad de tiempo desde el Cinturón Principal completo sobre la historia del Sistema Solar. Aquí, ilustramos la remoción de asteroides por acción del efecto Yarkovsky, la inyección colisional dentro de las resonancias $\nu_{6}, 3: 1$ y 5:2, y la eyección colisional más allá de las 3.27 UA. Sumando todos estos efectos, nuestras simulaciones sugieren una tasa media de remoción de 1070 asteroides con diámetros $D \geq 1 \mathrm{~km}$ por millón de años desde el Cinturón Principal. Este resultado es consistente con aquel derivado por. Bottke et al. (2002).

más importante que remueve material desde el Cinturón Principal de asteroides, más que la inyección colisional dentro de las resonancias principales, lo cual es consistente con los trabajos de Morbidelli et al. (2002) y Morbidelli \& Vokrouhlický (2003). En efecto, mientras que 891 asteroides con diámetros $D \geq 1 \mathrm{~km}$ son removidos por millón de años debido a la acción del efecto Yarkovsky, los procesos colisionales inyectan un total de 25, 38 y 68 asteroides mayores a $1 \mathrm{~km}$ por millón de años dentro de las resonancias $\nu_{6}, 3: 1$ y 5:2, respectivamente.

Estas tasas de remoción han sido obtenidas siguiendo el tratamiento dinámico propuesto en la Sección 5.6. A partir de esto, en cada paso de tiempo, una única órbita característica es generada al azar y luego utilizada en cada una de las poblaciones del modelo para objetos de todo tamaño. Para testear esta suposición, también desarrollamos diversas simulaciones donde, en cada paso de tiempo, diferentes órbitas fueron generadas al azar para cuerpos de un cierto diámetro en una dada población. Mientras que el tiempo de cómputo requerido resultó ser mucho más largo, los resultados no mostraron cambios relevantes. 


\subsubsection{Tiempo de Exposición a los Rayos Cósmicos de los Meteoritos}

El tiempo de exposición a los rayos cósmicos de un meteorito representa el intervalo durante el cual el material que lo constituye estuvo a una distancia menor a un metro por debajo de la superficie del objeto que lo albergó en el espacio, y su arribo a la Tierra. A partir de esto, notemos que el tiempo de exposición a los rayos cósmicos de los meteoritos sólo representa un límite inferior para el tiempo desde el cual el objeto fue eyectado de su cuerpo precursor.

De acuerdo a los análisis de Marti \& Graf (1992) y Morbidelli \& Gladman (1998), los tiempos de exposición a los rayos cósmicos para los diferentes tipos de condritas ordinarias, las cuales representan la clase más común de meteoritos, se encuentran en el rango que va desde unos pocos millones de años hasta $\sim 10^{8}$ años, con un valor medio de $\sim 1-2 \times 10^{7}$ años. Las diferentes subclases de condritas ordinarias muestran picos en diferentes edades. Particularmente, las condritas $\mathrm{H}$ presentan un pico prominente alrededor de los $8 \times 10^{6}$ años, el cual es posiblemente el resultado de un evento de impacto significativo. Welten et al. (1997) sugirieron que las acondritas tienen tiempos de exposición a los rayos cósmicos similares a aquellos de las condritas ordinarias, mientras que Caffee et al. (1988) indicaron que los tiempos de exposición a los rayos cósmicos para los meteoritos metálicos van desde $10^{8}$ a $10^{9}$ años. La Figura 5.14, extraída de Morbidelli \& Gladman (1998), muestra histogramas del tiempo de exposición a los rayos cósmicos para condritas ordinarias.

La distribución de tamaños de los meteoritos y los datos concernientes a sus tiempos de exposición a los rayos cósmicos indican que la mayoría de los cuerpos que alcanzan la superficie de la Tierra tienen tamaños preatmosféricos de $\sim 1 \mathrm{~m}$ (Farinella et al. 1998). La Figura 5.15 muestra los tiempos de vida colisionales medios obtenidos a partir de nuestras simulaciones. Para objetos con un diámetro $D=1 \mathrm{~m}$ pertenecientes a los Anillos Interior, Medio y Exterior del Cinturón Principal, estimamos tiempos de vida colisionales medios de $3.2,4.3$ y $6.8 \times 10^{6}$ años, respectivamente, los cuales son consistentes con la distribución de los tiempos de exposición a los rayos cósmicos de las condritas ordinarias (Figura 5.14). Además, nuestros resultados son compatibles con aquellos derivados por O'Brien \& Greenberg (2005), quienes obtuvieron un tiempo de vida colisional medio de $\sim 8 \times 10^{6}$ años para objetos con un diámetro $D=1 \mathrm{~m}$ pertenecientes al Cinturón Principal de asteroides.

\subsubsection{Historia Colisional del Asteroide (4) Vesta}

El asteroide (4) Vesta, con un diámetro $D \sim 500 \mathrm{~km}$, orbita el Sol a una distancia de $\sim 2.36$ UA. Este objeto representa uno de los casos más peculiares del Sistema Solar ya que es el único asteroide diferenciado conocido con una corteza casi intacta de composición basáltica, tal como lo indican los estudios espectroscópicos desarrollados por McCord et al. (1970). De acuerdo a lo sugerido por Davis et al (1985), la preservación de su corteza impone un límite sobre la intensidad de las colisiones que Vesta ha experimentado sobre la edad del Sistema Solar. En efecto, un modelo adecuado de evolución colisional del Cinturón Principal de asteroides debe minimizar la probabilidad de que objetos del tamaño de Vesta sufran una fragmentación catastrófica, ya que la misma podría quebrar la superficie y mezclar la corteza con el material de las capas inferiores de una forma tal que sería detectable espectroscópicamente.

Nuestras simulaciones indican que los asteroides con un diámetro $D \sim 500 \mathrm{~km}$ pertenecientes al Anillo Interior del Cinturón Principal, tienen un tiempo de vida colisional medio 

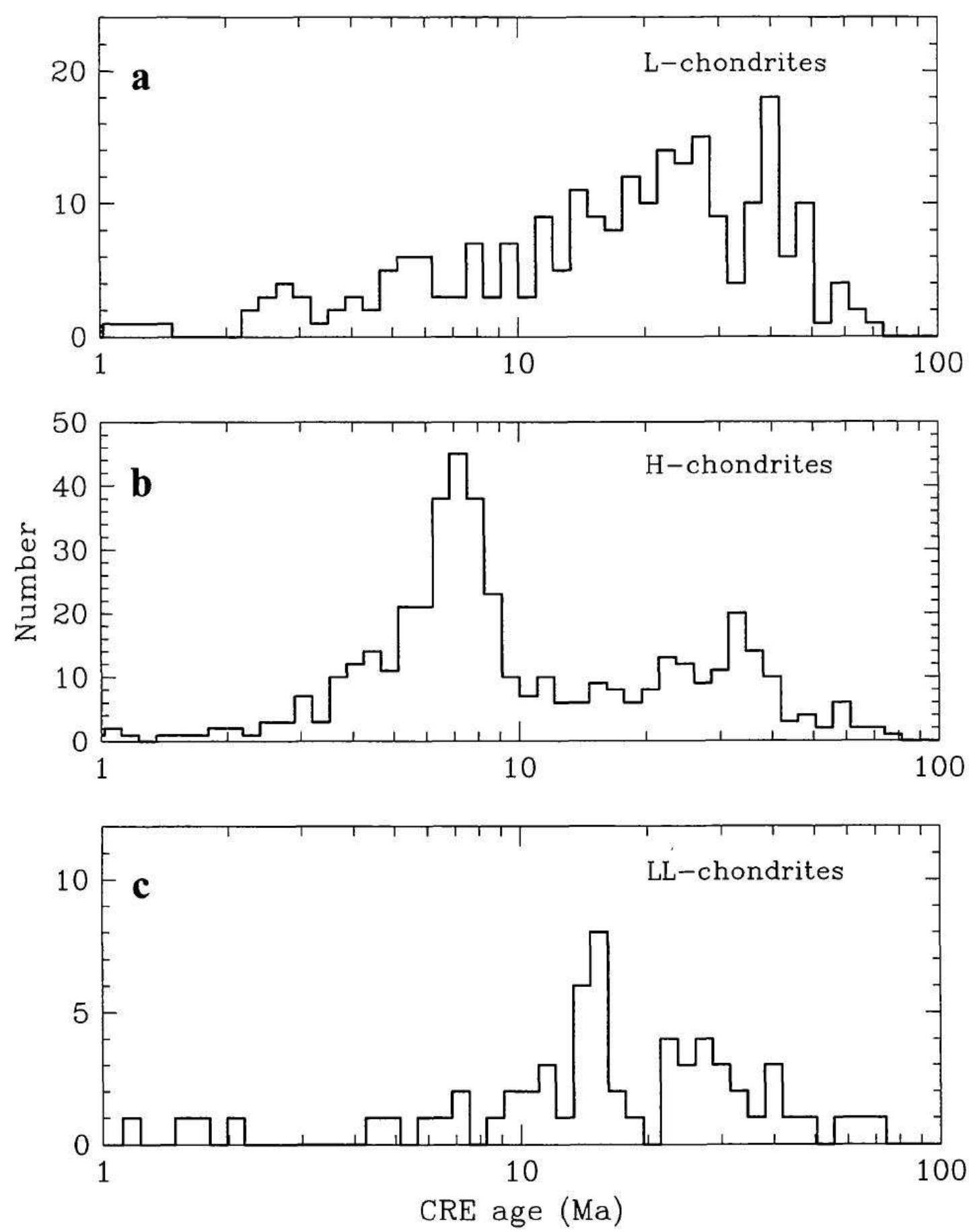

Figura 5.14. Histogramas del tiempo de exposición a los rayos cósmicos para diferentes tipos de condritas ordinarias. Figura extraída de Morbidelli \& Gladman (1998). 


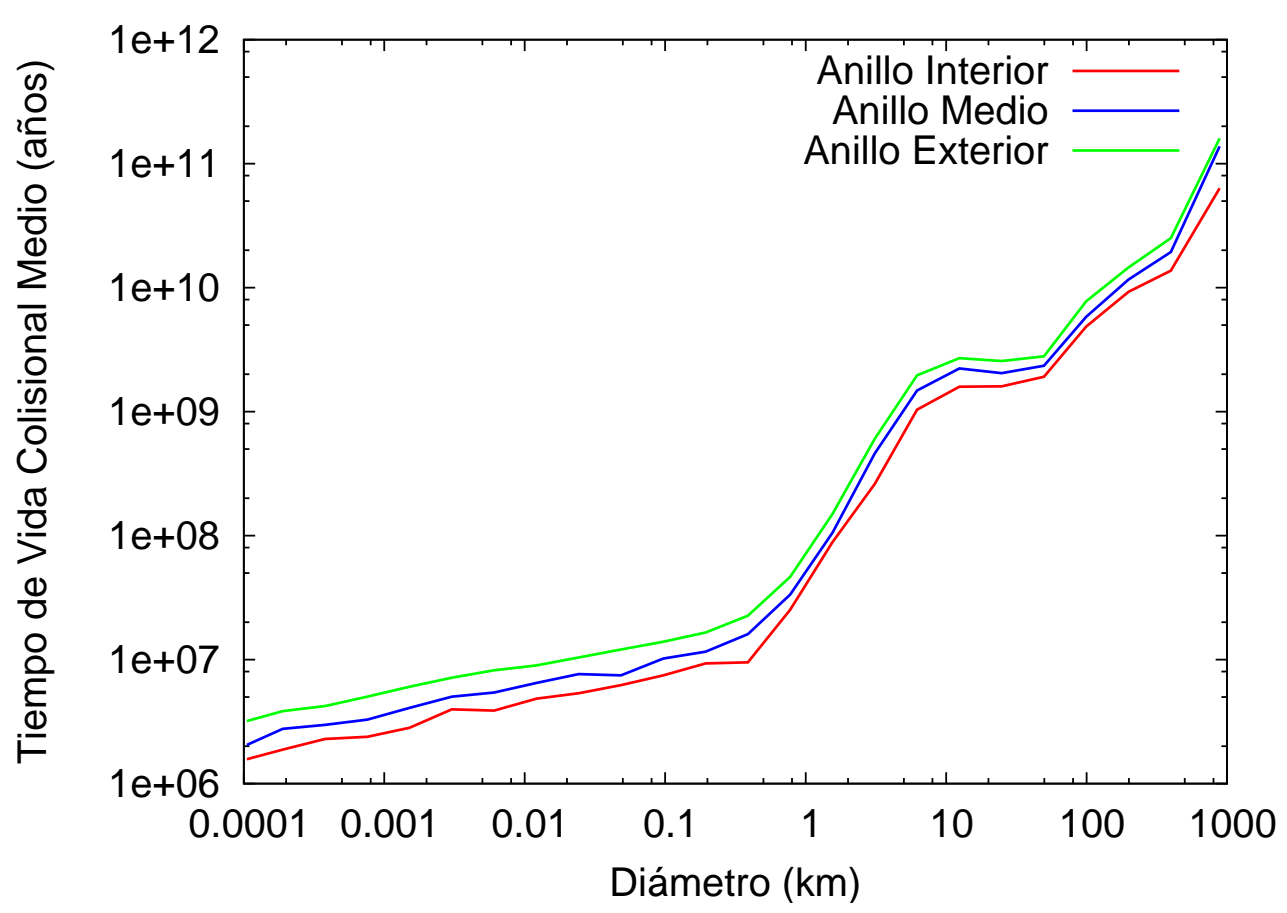

Figura 5.15. Tiempos de vida colisionales medios de objetos pertenecientes a los Anillos Interior, Medio y Exterior del Cinturón Principal. Los asteroides con un diámetro $D=1$ m presentan tiempos de vida colisionales medios entre 3.2 y $6.8 \times 10^{6}$ años, los cuales son consistentes con los tiempos de exposición a los rayos cósmicos de los meteoritos (Marti \& Graf 1992; Morbidelli \& Gladman 1998).

de $\sim 1.77 \times 10^{10}$ años. (Figura 5.15). Este resultado nos permite inferir que un objeto como Vesta tiene aproximadamente un $75 \%$ de probabilidad de sobrevivir sobre la edad del Sistema Solar sin recibir un impacto catastrófico, lo cual es consistente con la preservación de la corteza basáltica de dicho asteroide.

Por otra parte, observaciones realizadas con el Telescopio Espacial Hubble han revelado que la superficie de Vesta posee un cráter singular, cuyo diámetro es de $\sim 450 \mathrm{~km}$ (Figura 5.16). De acuerdo a estudios desarrollados por Thomas et al. (1997), este cráter se produjo como consecuencia de un evento de impacto que Vesta experimentó con un objeto de $\sim 35 \mathrm{~km}$ de diámetro.

Bottke et al. (2005b) utilizaron el hecho de que Vesta no tenga dos de estas estructuras como una restricción a su estudio de evolución colisional. Nuestro modelo también nos permite analizar cuantitativamente las colisiones sufridas sobre Vesta por objetos de $\sim 35 \mathrm{~km}$ de diámetro, a lo largo de la historia del Sistema Solar. Para hacer esto, estimamos el número de proyectiles $N$ con diámetros $D_{\mathrm{p}} \sim 35 \mathrm{~km}$, y computamos el intervalo medio de impacto entre tales objetos y Vesta a partir de la expresión

$$
\tau=\sum \frac{4}{\left\langle P_{\mathrm{i}}\right\rangle\left(D_{\text {Vesta }}+D_{\mathrm{p}}\right)^{2} N},
$$

donde la suma se extiende sobre todas las poblaciones del modelo, y $\left\langle P_{\mathrm{i}}\right\rangle$ representa la probabilidad intrínseca de colisión correspondiente. Nuestros resultados indican que existe $\sim 50 \%$ 


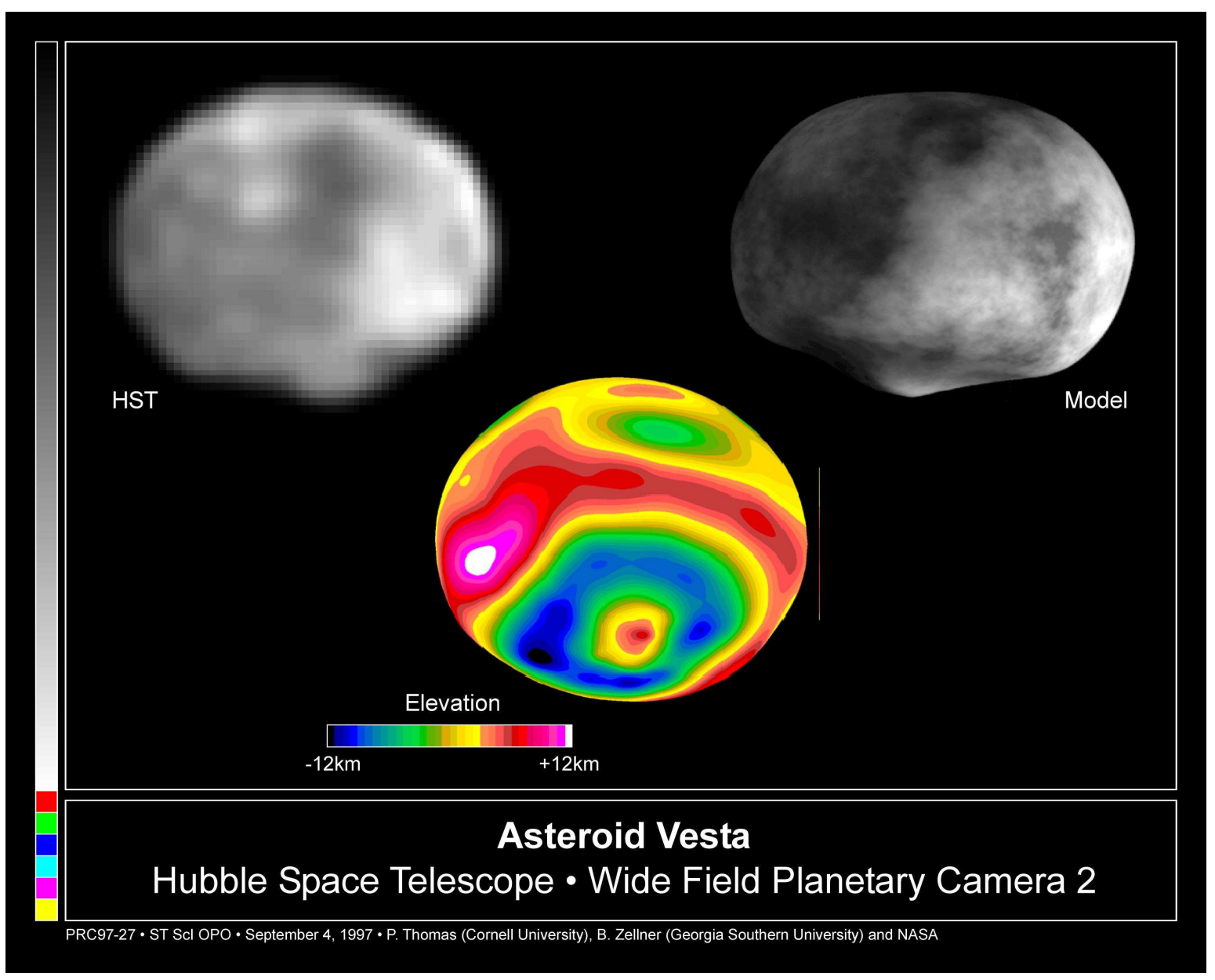

Figura 5.16. A la izquierda, se observa una fotografía del asteroide (4) Vesta tomada por el Telescopio Espacial Hubble en Mayo de 1996, cuando este se encontraba a 1.18 UA de la Tierra. En el centro, se muestra un mapa a color de la elevación de la superficie de Vesta. Es evidente la existencia de un cráter de impacto en el hemisferio sur, cuyo diámetro es de $\sim 450 \mathrm{~km}$. A la derecha, se observa un modelo de computadora de Vesta, realizado a partir de los datos topográficos del Hubble. El pico central del cráter puede verse claramente cerca del polo. (Crédito de la Imagen: Ben Zellner, Peter Thomas y NASA). 
de probabilidad de que un objeto con un diámetro $D_{\mathrm{p}} \sim 35 \mathrm{~km}$ colisione con Vesta sobre los $4.5 \times 10^{9}$ años de evolución. Siguiendo el razonamiento de Bottke et al. (2005b), este resultado es consistente con la existencia de un único cráter singular observado sobre Vesta, y aun más, con el hecho de no contar con una segunda estructura de impacto de tamaño comparable.

\subsubsection{Familias de Asteroides}

La existencia de familias de asteroides en el espacio de los elementos propios ha sido reconocida desde el trabajo pionero de Hirayama (1918), hace más de noventa años. Una familia de asteroides es el resultado de un evento de impacto experimentado por un mismo cuerpo precursor. En efecto, los miembros de una familia son fragmentos colisionales con un origen común.

Los elementos orbitales instantáneos experimentan oscilaciones de largo y corto período debido a las perturbaciones planetarias. Dichas oscilaciones no nos permiten una buena visualización de las familias de asteroides. Para distinguir tales agrupamientos, la herramienta más adecuada viene dada por los elementos propios. En efecto, estos elementos eliminan las oscilaciones de largo y corto período debidas a las perturbaciones planetarias, siendo prácticamente constantes en el tiempo. De este modo, los fragmentos generados a partir de la fractura de un dado objeto se ubicarán en regiones muy cercanas en el espacio de los elementos propios (a,e) y $(a, i)$, poniendo en evidencia los agrupamientos mencionados. La Figura 5.17 representa la distribución de los asteroides numerados en el espacio de los elementos propios, obtenidos de la base de datos de Knežević \& Milani (2000). A partir de la misma, diversas familias de asteroides pueden ser fácilmente visualizadas.

La existencia de familias de asteroides representa una clara consecuencia de la actividad colisional en el Cinturón Principal. De acuerdo al trabajo desarrollado por Zappalà et al. (1995), existen aproximadamente 25 familias confiables en el espacio de los elementos propios. Además, también es posible identificar un total de aproximadamente 60 grupos de asteroides estadísticamente significativos.

Davis et al. (1985) indicaron que la existencia de familias de asteroides impone una restricción sobre la historia colisional del Cinturón Principal. En efecto, ellos sugirieron la existencia de 8 familias en el cinturón de asteroides actual, formadas a partir de cuerpos mayores a los $200 \mathrm{~km}$ de diámetro. Nuestras simulaciones predicen exactamente la formación de 8 familias de asteroides en el Cinturón Principal, generadas a partir de cuerpos precursores con diámetros $D>200 \mathrm{~km}$. Además, es importante tener en cuenta que esas 8 familias generadas en nuestras simulaciones se forman después del breve período de evolución primordial de $5 \times$

$10^{6}$ años, el cual es incluido para modelar la existencia de un cinturón de asteroides primitivo masivo.

Por otra parte, los estudios de familias de asteroides desarrollados por Vokrouhlický et al. (2006) muestran que la velocidad de eyección típica de fragmentos con un diámetro $D \sim$ $5 \mathrm{~km}$ es del orden de unas pocas decenas de metros por segundo. Siguiendo las sugerencias de O’Brien \& Greenberg (2005), nosotros asumimos un valor máximo para la velocidad de eyección de los fragmentos resultantes de una colisión de $3000 \mathrm{~m} \mathrm{~s}^{-1}$, el cual es del orden de la velocidad del sonido en el material. Si bien este valor parecería ser demasiado grande, nuestros estudios indican que aproximadamente el $90 \%$ de los fragmentos de $5 \mathrm{~km}$ de diámetro son 

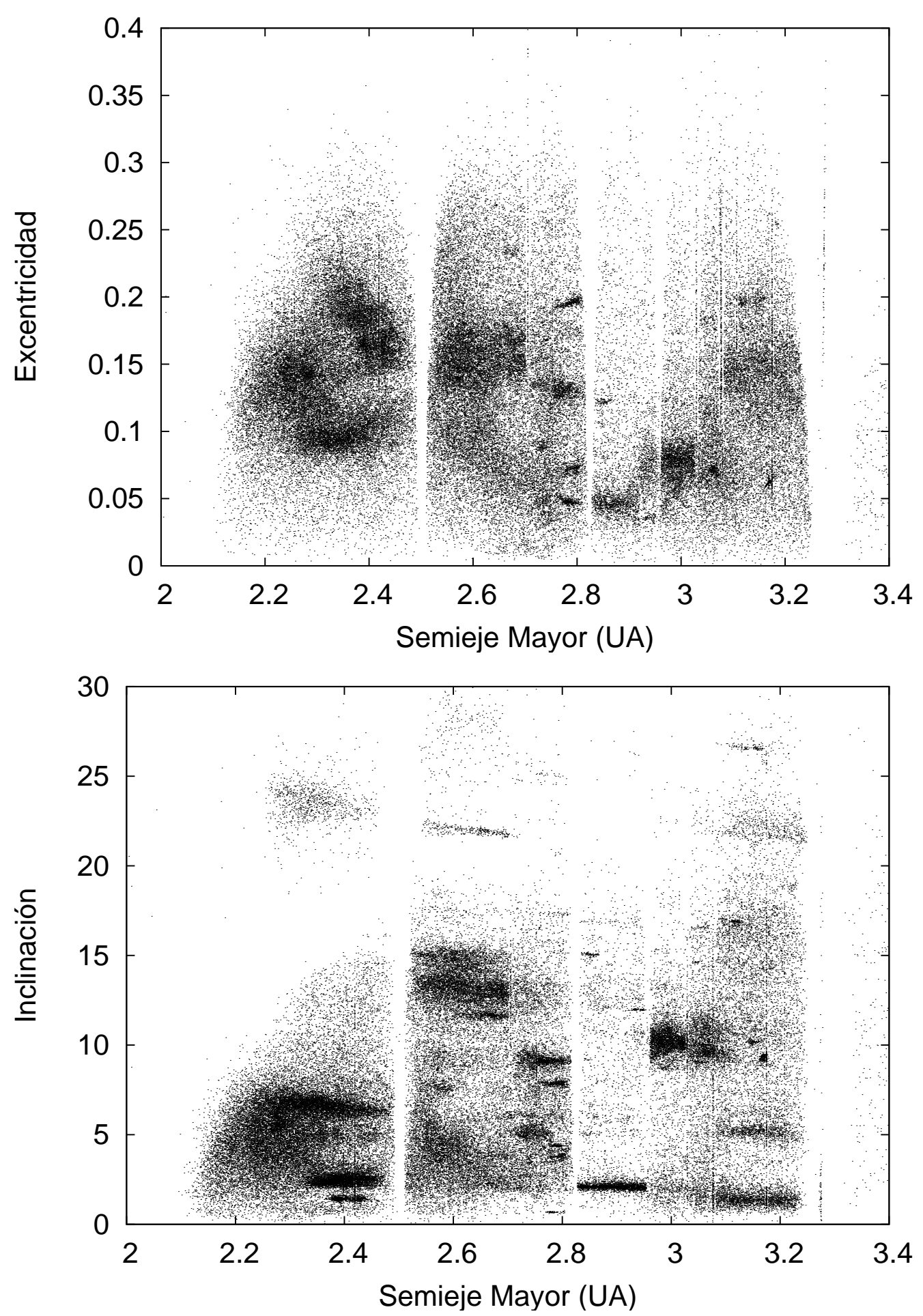

Figura 5.17. Elementos propios $a$, e e $i$ para asteroides numerados, obtenidos de la base de datos de Knežević \& Milani (2000). Las familias de asteroides resultan ser claramente visibles. 


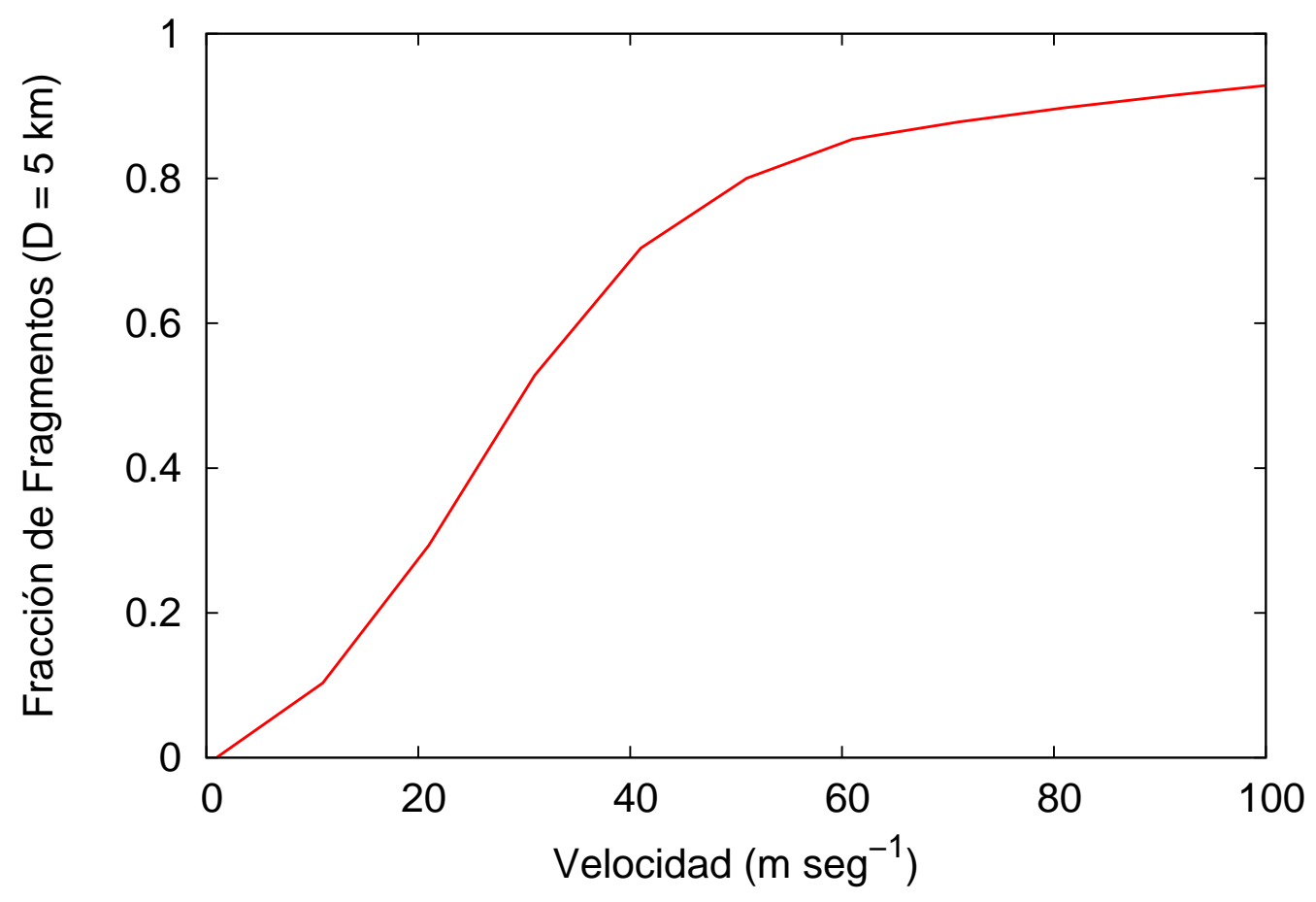

Figura 5.18. Fracción acumulada de fragmentos de $\sim 5 \mathrm{~km}$ de diámetro como una función de la velocidad. Nuestros estudios indican que aproximadamente el $90 \%$ de tales fragmentos son eyectados con velocidades menores a los $80 \mathrm{~m} \mathrm{~s}^{-1}$, lo cual es consistente con aquello discutido por Vokrouhlický et al. (2006).

eyectados con velocidades menores a los $80 \mathrm{~m} \mathrm{~s}^{-1}$ (Figura 5.18), lo cual es consistente con aquello discutido por Vokrouhlický et al. (2006).

\subsubsection{Mezcla de Clases Taxonómicas - Discusión}

Desde hace algunas décadas, la distribución de clases taxonómicas en el Cinturón Principal de asteroides ha sido ampliamente estudiada por muchos autores. Tal como describimos en la Sección 3.2.1, el trabajo desarrollado por Gradie \& Tedesco (1982) ha sido aceptado como la principal referencia concerniente a la distribución y mezcla de clases taxonómicas. Sin embargo, Mothé-Diniz et al. (2003) refinaron la distribución heliocéntrica de taxonomías en el Cinturón Principal de asteroides, encontrando importantes diferencias con el análisis realizado por Gradie \& Tedesco (1982) y otros trabajos previos.

Conociendo la existencia de esta distribución de taxonomías, el objetivo de nuestro análisis es determinar si tal distribución de clases es un rasgo característico del proceso de formación del Cinturón Principal o podría haber cambiado sobre la evolución del Sistema Solar. La Figura 5.19 muestra que, después de $4.5 \times 10^{9}$ años de evolución, más del $99 \%$ de la masa final de cada uno de los tres anillos en los que hemos dividido el cinturón de asteroides está representado por material primordial. A partir de esto, nosotros concluimos que la distribución y mezcla de clases taxonómicas observada en el Cinturón Principal de asteroides no puede ser explicada por el intercambio colisional de masa. A partir de esto, tal distribución de 
taxonomías debería ser un rasgo primordial del Cinturón Principal, asociado al proceso de formación de dicha estructura.

En este estudio, el transporte de material entre las diferentes regiones del Cinturón Principal debido a la acción del efecto Yarkovsky no ha sido tenido en cuenta. Con el objetivo de justificar esta suposición, debemos analizar nuestro modelo del efecto Yarkovsky junto con las propiedades dinámicas de las resonancias poderosas $\nu_{6}, 3: 1$ y 5:2, las cuales determinan los límites de los tres anillos del Cinturón Principal. La Figura 5.7 muestra que la tasa de cambio del semieje mayor $\dot{a}$ para cuerpos con diámetros $D \geq 10^{-4} \mathrm{~km}$ es siempre menor a $0.005 \mathrm{UA}$ por millón de años para cualquiera de los tres anillos del cinturón de asteroides. Además, tal como indica la Tabla 3.1 de la Sección 3.1.2, el tiempo de vida medio de los cuerpos inicialmente en las resonancias $\nu_{6}$ y $3: 1$ es de $\sim 2 \times 10^{6}$ años mientras que, para la resonancia 5:2, el tiempo de vida medio es de $\sim 0.5 \times 10^{6}$ años. Asumiendo que estas resonancias poderosas tienen un ancho característico de algunas centésimas de UA, el tiempo requerido para cruzar estas regiones es siempre mayor que los tiempos de vida medios anteriormente mencionados. De este modo, nosotros consideramos que el efecto Yarkovsky no juega un rol importante en mezclar material entre las diferentes zonas del Cinturón Principal.

\subsubsection{Origen de los NEAs}

Aquí, nosotros estudiamos qué porcentaje de la masa de la población de NEAs proviene desde las diferentes regiones del Cinturón Principal. En cada paso de tiempo, computamos las tasas de inyección desde cada uno de los tres anillos del cinturón de asteroides dentro de la población de NEAs, y las tasas de eliminación dinámica de aquellos cuerpos desde esta población. Para calcular las tasas de inyección, tenemos en cuenta la acción del efecto Yarkovsky y la inyección colisional dentro de las resonancias $\nu_{6}, 3: 1$ y 5:2. Para computar la tasa de remoción de objetos desde la población de NEAs, necesitamos determinar el tiempo de vida dinámico medio de los cuerpos en esta población. De acuerdo a lo analizado por Bottke et al. (2002), obtenemos un tiempo de vida dinámico medio de $3.73 \times 10^{6}$ años en la región de los NEAs para objetos provenientes de los anillos Interior y Medio del Cinturón Principal, promediando los valores asociados a la resonancia secular $\nu_{6}$, la población principal de Cruzadores de Marte (IMC) y la resonancia de movimientos medios 3:1 con Júpiter. Por otra parte, para cuerpos provenientes del anillo Exterior del Cinturón Principal, asumimos un tiempo de vida dinámico medio de $1.4 \times 10^{5}$ años (Bottke et al., 2002; Tabla 3.1). Nuestro análisis muestra que aproximadamente el $94 \%$ de la población de NEAs proviene desde los anillos Interior y Medio del Cinturón Principal mientras que el $6 \%$ restante proviene desde el anillo Exterior, lo cual es consistente con aquello discutido por Bottke et al. (2002) quienes encontraron que aproximadamente el $85 \%$ de la población de NEAs proviene de los anillos Interior y Medio del cinturón de asteroides (esto es, $a<2.8 \mathrm{UA}$ ), el $8 \%$ del Cinturón Principal Exterior y el $6 \%$ restante de la población de Cometas de la Familia de Júpiter. 

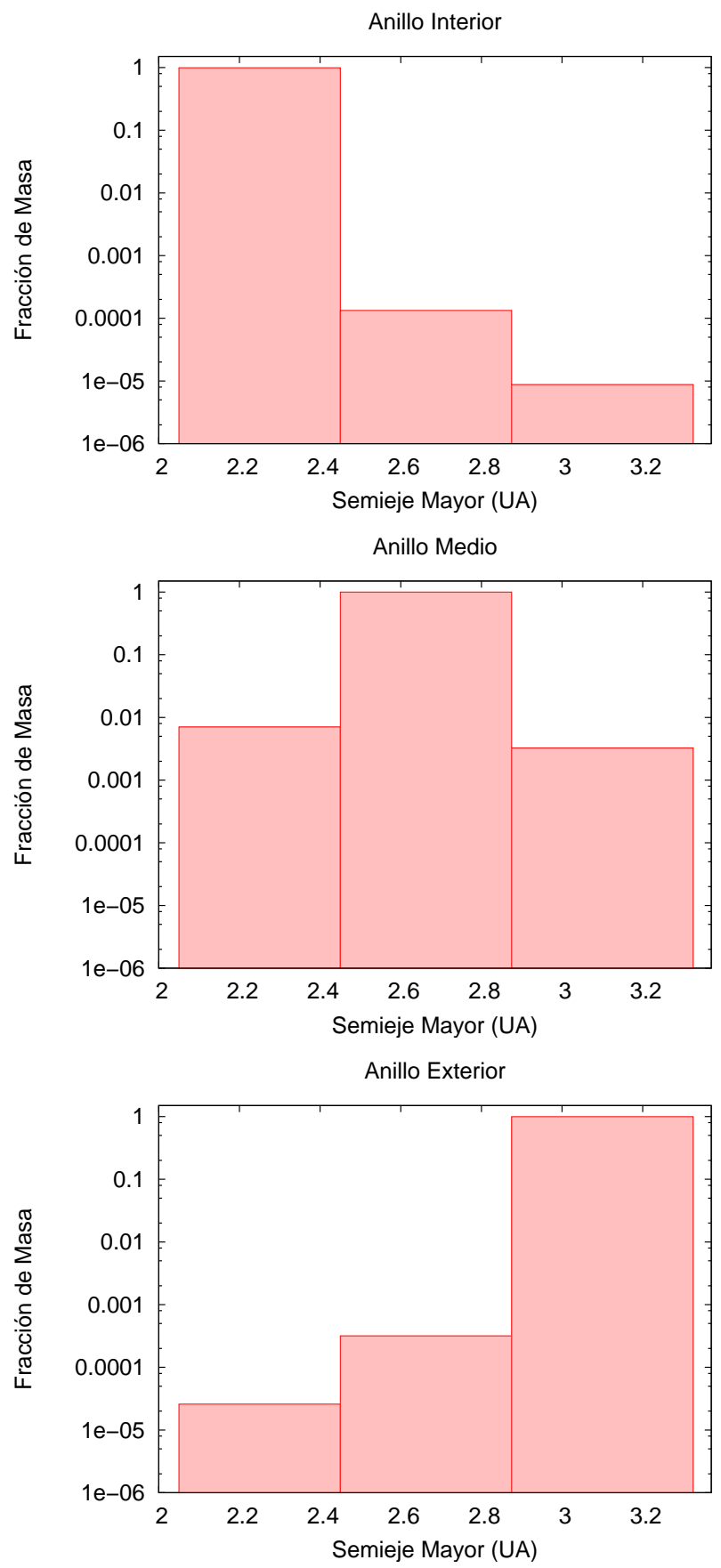

Figura 5.19. Fracción de masa asociada a los Anillos Interior, Medio y Exterior distribuida en el Cinturón Principal completo, debido a la acción de los procesos colisionales, después de $4.5 \times 10^{9}$ años de evolución. Cada anillo conserva más del $99 \%$ de su masa primordial, lo cual nos permite inferir que la mezcla de clases taxonómicas observada en el cinturón de asteroides no puede ser explicada por el intercambio colisional de material. 



\section{Capítulo 6}

\section{Troyanos de Júpiter}

\subsection{Introducción}

Los resultados presentados en este capítulo, con excepción de aquellos discutidos en la Sección 6.6.4 concernientes al polvo Troyano, fueron publicados en Astronomy \& Astrophysics, y pueden encontrarse bajo la referencia de Elía \& Brunini (2007b).

Los primeros trabajos destinados a comprender las propiedades dinámicas de los asteroides Troyanos en el marco del problema restringido de los tres cuerpos fueron desarrollados por Szebehely (1967) y Rabe (1967). En efecto, Szebehely (1967) realizó una descripción detallada de tal problema incluyendo regularización, puntos de equilibrio, órbitas periódicas y su estabilidad, y curvas de Hill. Al mismo tiempo, Rabe (1967) analizó las libraciones de largo período de los Troyanos y definió límites de estabilidad en el espacio amplitud de libraciónexcentricidad. Algunos años más tarde, Érdi (1978) estudió analíticamente el movimiento tridimensional de los asteroides Troyanos en el marco del problema restringido elíptico de los tres cuerpos, e investigó los principales efectos de las perturbaciones de Júpiter sobre los elementos orbitales de estos objetos.

El comportamiento dinámico de la población de Troyanos jovianos ha sido también ampliamente estudiado por métodos numéricos. Basados en una muestra de 40 Troyanos, Bien \& Schubart (1987) describieron la construcción de tres elementos propios - la amplitud de libración, la excentricidad propia y la inclinación propia - los cuales resultaron ser constantes sobre, al menos, $\sim 10^{5}$ años. Más tarde, Schubart \& Bien (1987) analizaron la distribución de estas cantidades y su relación con otros parámetros dinámicos. En los primeros años de la década del 90, Milani (1993) integró numéricamente las órbitas de 174 Troyanos por $10^{6}$ años, teniendo en cuenta la influencia gravitacional de los cuatro planetas gigantes. Este trabajo fue capaz de computar elementos propios precisos y estables, permitiendo detectar algunos agrupamientos, candidatos a ser familias de asteroides. Más tarde, Levison et al. (1997) desarrollaron simulaciones numéricas con el objetivo de estudiar la estabilidad de los asteroides Troyanos bajo las perturbaciones de todos los planetas exteriores, sobre escalas de tiempo de $\sim 10^{9}$ años. Usando un código numérico de $N$ cuerpos, Levison et al. (1997) mostraron que los Troyanos se mueven sobre órbitas que no resultan ser estables indefinidamente, indicando que la influencia gravitacional de los planetas gigantes ha reducido los límites exteriores de los enjambres en el tiempo. En efecto, el resultado más importante obtenido por estos autores 
es que las nubes de asteroides Troyanos jovianos asociadas a los puntos $L_{4}$ y $L_{5}$ se están dispersando lentamente, estimando una tasa de erosión dinámica de $\sim 6.2 \times 10^{-5}$ objetos con diámetros $D \gtrsim 1 \mathrm{~km}$ por año.

Otra cuestión importante concerniente a los asteroides Troyanos jovianos es su historia colisional. El primer intento de estudiar la evolución colisional de estos objetos fue llevada a cabo por Davis \& Weidenschilling (1981), quienes derivaron velocidades medias de impacto e intervalos de tiempo entre colisiones catastróficas para Troyanos de diferentes tamaños. En efecto, estos autores encontraron una velocidad media de colisión para Troyanos de $\sim 3.5$ $\mathrm{km} \mathrm{s}^{-1}$, basados en una muestra de sólo 69 objetos. Por otra parte, ellos sugirieron que los asteroides Troyanos de mayor tamaño representan una parte de la población que probablemente ha sobrevivido inalterada por impactos catastróficos sobre la edad del Sistema Solar.

Contando con elementos propios precisos de una muestra de 174 Troyanos, Milani (1993, 1994) identificó tres familias confiables: las familias Menelaus y Teucer en el punto $L_{4}$, y la familia Sarpedon en $L_{5}$. La existencia de estas familias y de otros agrupamientos de asteroides más pequeños permitieron confirmar que la población de Troyanos jovianos ha experimentado una actividad colisional significativa. Más tarde, Marzari et al. (1995) estudiaron la formación de familias colisionales de Troyanos jovianos y analizaron una posible conexión entre los fragmentos generados por colisiones entre Troyanos y la población de Cometas de Corto Período. En efecto, estos autores mostraron que el 20\% de tales fragmentos finalizan en órbitas inestables teniendo encuentros cercanos con Júpiter, sugiriendo que unas pocas decenas de los Cometas de Corto Período observados podrían haberse originado por colisiones entre Troyanos.

En la segunda mitad de la década del 90, Marzari et al. (1996) desarrollaron un algoritmo numérico destinado a calcular tasas de colisión y velocidades de impacto para los asteroides Troyanos. Basados en una muestra de 114 Troyanos, estos autores computaron los valores de estos parámetros colisionales sobre $\sim 10^{4}$ años, estimando probabilidades intrínsecas de colisión para los puntos $L_{4}$ y $L_{5}$, y una velocidad media de impacto de $\sim 5 \mathrm{~km} \mathrm{~s}^{-1}$, la cual es un poco más elevada que aquella derivada por Davis \& Weidenschilling (1981). Usando estos parámetros colisionales, Marzari et al. (1997) modelaron la evolución colisional de los asteroides Troyanos jovianos. Estos autores encontraron que varias decenas de familias de Troyanos son generadas a partir de cuerpos precursores mayores a los $60 \mathrm{~km}$ y sugirieron que el flujo de fragmentos colisionales que finalizan en órbitas cometarias podría suministrar el $10 \%$ de la población de Centauros y Cometas de Corto Período. Más tarde, Dell'Oro et al. (1998) desarrollaron un método estadístico destinado a computar la probabilidad de colisión y la velocidad de impacto en las dos nubes de Troyanos, sobre una escala de tiempo mayor que aquella usada por Marzari et al. (1996). Basado en un conjunto de 223 Troyanos, Dell'Oro et al. (1998) estimaron probabilidades intrínsecas de colisión para los puntos $L_{4}$ y $L_{5}$, y una velocidad media de impacto de $\sim 4.5 \mathrm{~km} \mathrm{~s}^{-1}$.

Años más tarde, Gil-Hutton \& Brunini (2000) simularon numéricamente la interacción colisional entre el Cinturón de Asteroides exterior y los planetesimales primordiales dispersados de la zona de Urano y Neptuno durante el período de acreción. Ellos mostraron que las distribuciones de tamaños finales de los Troyanos e Hildas están dominadas por el bombardeo de cometesimales, el cual ocurre durante los primeros $2 \times 10^{7}$ años de evolución (Brunini \& Fernández 1999).

Al mismo tiempo, Jewitt et al. (2000) presentaron un análisis de la población y distribución 
de tamaños de los asteroides Troyanos jovianos pequeños, desarrollado a partir de un estudio óptico tomado en la dirección del punto $L_{4}$. Estos autores estimaron que el número de Troyanos de $L_{4}$ con radios $R>1 \mathrm{~km}$ es de $\sim 1.6 \times 10^{5}$. Además, ellos argumentaron que un radio crítico de $\sim 30-40 \mathrm{~km}$ podría marcar un tamaño de transición entre objetos primordiales y fragmentos colisionales. Algunos años más tarde, Yoshida \& Nakamura (2005) detectaron 51 asteroides Troyanos jovianos en el punto $L_{4}$ con diámetros $D$ en el rango $0.7 \lesssim D \lesssim 12.3$ $\mathrm{km}$. Estos autores determinaron la distribución de tamaños de los Troyanos para $2 \lesssim D \lesssim 10$ $\mathrm{km}$, encontrando resultados consistentes con aquellos derivados previamente por Jewitt et al. (2000). Sin embargo, Yoshida \& Nakamura (2005) notaron un suave quiebre en la distribución de tamaños a un diámetro de $\sim 5 \mathrm{~km}$.

Por otra parte, Beaugé \& Roig (2001) desarrollaron un modelo semianalítico para el movimiento de los asteroides Troyanos, destinado a estudiar el comportamiento dinámico de estos objetos sobre escalas de tiempo del orden de $10^{7}$ años. Haciendo uso de este algoritmo, estos autores estimaron elementos propios precisos para una muestra de 533 Troyanos, lo cual les permitió realizar una búsqueda de familias alrededor de los puntos $L_{4}$ y $L_{5}$. En efecto, mientras que Beaugé \& Roig (2001) confirmaron la existencia de la familia Menelaus en $L_{4}$, previamente detectada por Milani $(1993,1994)$, ellos pusieron en duda la familia Teucer encontrada por este último, proponiendo a la familia Epeios como una candidata más confiable a ser el resultado de un evento colisional a partir de un mismo objeto precursor. Además, Beaugé \& Roig (2001) no identificaron ninguna familia alrededor del punto $L_{5}$, sugiriendo una posible asimetría entre los dos enjambres. Algunos años más tarde, Fornasier et al. (2004), Dotto et al. (2006) y Fornasier et al. (2007) llevaron a cabo análisis fotométricos y espectroscópicos de asteroides Troyanos. Estos estudios, destinados a analizar las propiedades mineralógicas de los pequeños y grandes miembros de las diferentes familias dinámicas, permitieron investigar la naturaleza de estos grupos y la composición interna de sus cuerpos precursores. En efecto, haciendo uso de la lista de familias de Troyanos jovianos provista por Beaugé \& Roig (2001), Fornasier et al. (2004) desarrollaron un estudio fotométrico y espectroscópico alrededor del punto $L_{5}$, estudiando las propiedades de diversos miembros pertenecientes a las familias Aneas, Astyanax, Sarpedon y Phereclos. Por otra parte, Dotto et al. (2006) realizaron un estudio óptico e infrarrojo de los puntos $L_{4}$ y $L_{5}$, investigando las propiedades superficiales de diversos miembros pertenecientes a siete familias dinámicas de ambos enjambres. En efecto, estos autores concentraron su atención sobre las cuatro familias de $L_{5}$ estudiadas por Fornasier et al. (2004) y analizaron las familias Menelaus, 1986 WD y Makhaon asociadas a $L_{4}$. El resultado más importante derivado por Dotto et al. (2006) es la uniformidad de la población de Troyanos. Recientemente, Fornasier et al. (2007) presentaron resultados finales sobre familias dinámicas a partir de un estudio óptico realizado sobre los enjambres jovianos $L_{4}$ y $L_{5}$. Estos autores estudiaron las principales características de los pequeños y grandes miembros de las familias de $L_{5}$ Aneas, Anchises, Misenus, Phereclos, Sarpedon y Panthoos, y de las familias Eurybates, Menelaus, 1986 WD y 1986 TS6 asociadas al punto $L_{4}$.

Tal como fue anticipado al presentar el trabajo de Marzari et al. (1997), otro de los puntos de interés relevante en el estudio de los Troyanos jovianos es la posible relación entre estos objetos y las poblaciones de Centauros y Cometas de la Familia de Júpiter. La existencia de alguna conexión genética entre los asteroides Troyanos jovianos y los Cometas de Corto Período fue sugerida inicialmente por Hartmann et al. (1987), Shoemaker et al. (1989), Jewitt \& Luu (1990) y Fitzsimmons et al. (1994), quienes desarrollaron estudios espectroscópicos y encontraron similitudes entre los cometas y los asteroides tipo $D$, los cuales representan la 
clase más abundante entre los Troyanos jovianos. Por otra parte, de acuerdo a lo discutido en la Sección 3.2.2, la distribución de albedos de los Troyanos de Júpiter resulta ser consistente con aquella de los cometas (Fernández et al. 2003).

Aquí, nosotros presentamos un estudio destinado a analizar la evolución colisional y dinámica de los asteroides Troyanos jovianos de $L_{4}$. Si bien este trabajo tiene características similares al desarrollado por Marzari et al. (1997), existen diferencias relevantes en el tratamiento general del algoritmo. En cuanto al modelo colisional, Marzari et al. (1997) modelaron $Q_{\mathrm{S}}$ a partir de las leyes formuladas por Davis et al. (1985), Housen \& Holsapple (1990) y Davis et al. (1994), y asumieron un valor constante de 0.2 para el parámetro $f_{\text {ke. Aquí, nosotros }}$ usamos diferentes leyes $Q_{\mathrm{S}}$ y un parámetro $f_{\mathrm{ke}}$ dependiente del tamaño del blanco (Davis et al. 1995), los cuales son combinados para producir la ley $Q_{\mathrm{D}}$ formulada por Benz \& Asphaug (1999) para cuerpos de hielo a velocidades de $3 \mathrm{~km} \mathrm{~s}^{-1}$. Por otra parte, nuestro modelo mejora aquel propuesto por Marzari et al. (1997), incluyendo un tratamiento dinámico que tiene en cuenta las regiones de estabilidad e inestabilidad del punto $L_{4}$. Esto nos permite obtener estimaciones más confiables de la tasa de eyección colisional de fragmentos de Troyanos y discutir su posible contribución a las poblaciones actuales de Centauros y Cometas de la Familia de Júpiter.

\subsection{Población Inicial}

Con el objetivo de simular la evolución colisional y dinámica de los asteroides Troyanos jovianos del punto de equilibrio $L_{4}$, nuestro código numérico evoluciona en el tiempo el número de cuerpos residiendo en un conjunto de 130 bines de tamaño, cuyos valores centrales se encuentran en el rango que va desde $D_{1}=10^{-10} \mathrm{~km}$ a $D_{130}=886.7 \mathrm{~km}$ de diámetro en una forma tal que desde un bin al siguiente, la masa de los cuerpos cambia en un factor 2 y el diámetro se modifica en un factor $2^{1 / 3}$. Para nuestras simulaciones, adoptamos una densidad de $1.5 \mathrm{~g} \mathrm{~cm}^{-3}$, la cual es consistente con los diferentes estudios concernientes a la densidad de los Troyanos jovianos, previamente discutidos en la Sección 3.2.2.

La población y distribución de tamaños de los asteroides Troyanos jovianos fue estudiada por Jewitt et al. (2000), quienes desarrollaron un estudio óptico en la dirección del enjambre $L_{4}$, haciendo uso del telescopio de $2.2 \mathrm{~m}$ de la Universidad de Hawaii. Estos autores encontraron 93 Troyanos jovianos, con tamaños en el rango de $4 \lesssim D_{0,04} \lesssim 40 \mathrm{~km}$, siendo $D_{0,04}$ el diámetro derivado asumiendo un albedo geométrico de 0.04, el cual, de acuerdo a lo discutido en la Sección 1.3.3., es el valor medio de los Troyanos conocidos (Tedesco 1989; Fernández et al. 2003). De acuerdo a este análisis, la distribución de tamaños diferencial de los Troyanos jovianos de $L_{4}$ puede ser calculada a partir de las siguientes expresiones:

$$
n_{1}\left(D_{0,04}\right) d D_{0,04}=1,2 \times 10^{7}\left(\frac{1 \mathrm{~km}}{D_{0,04}}\right)^{3,0 \pm 0,3} d D_{0,04}
$$

para $4.4 \leq D_{0,04} \leq 40 \mathrm{~km}, \mathrm{y}$

$$
n_{2}\left(D_{0,04}\right) d D_{0,04}=1,6 \times 10^{11}\left(\frac{1 \mathrm{~km}}{D_{0,04}}\right)^{5,5 \pm 0,9} d D_{0,04}
$$



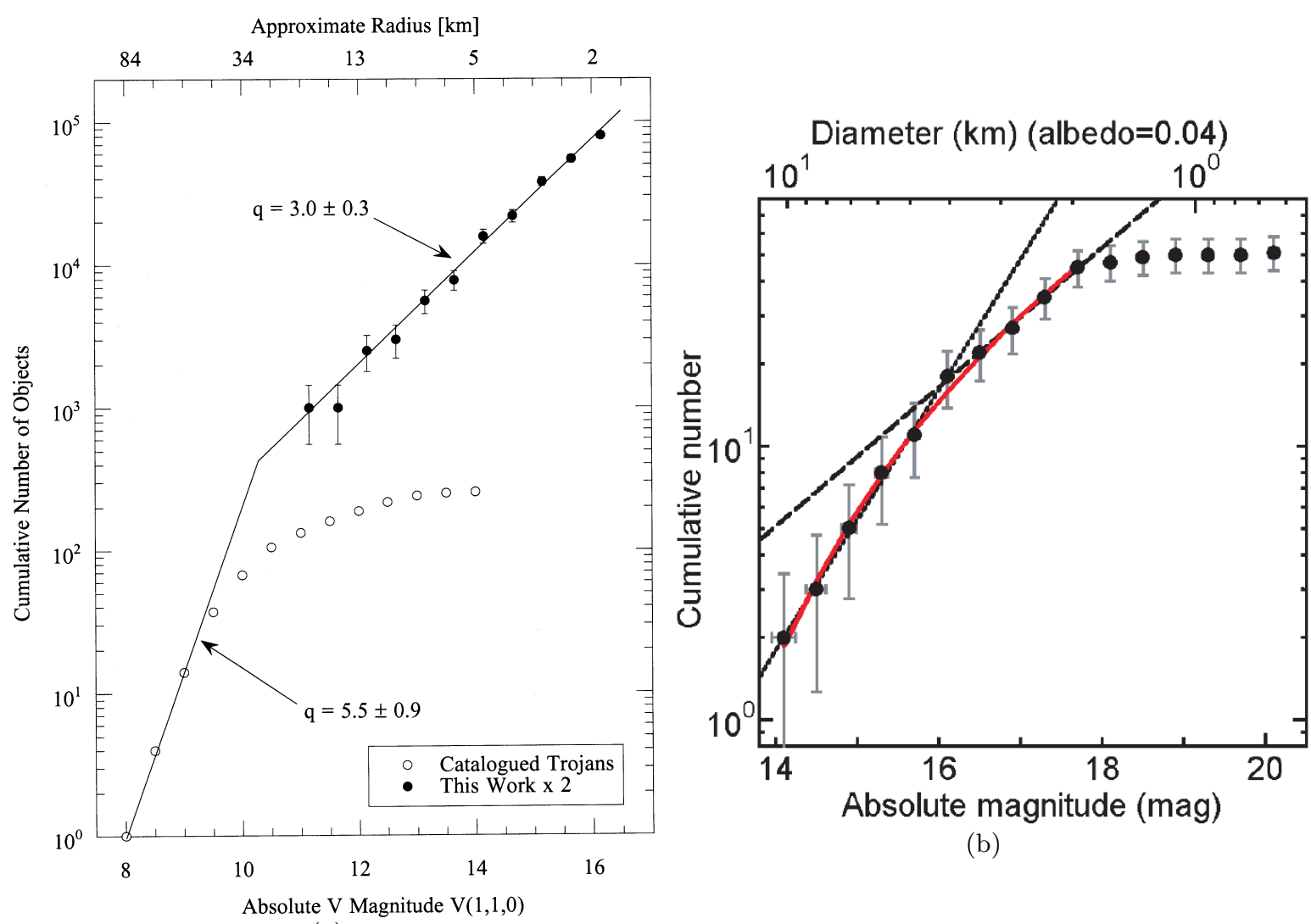

(b)

(a)

Figura 6.1. Distribuciones de tamaños de los Troyanos jovianos de $L_{4}$ derivadas por Jewitt et al. (2000) a) y Yoshida \& Nakamura (2005) b).

para $D_{0,04} \geq 84 \mathrm{~km}$, mientras que la correspondiente distribución de tamaños acumulada, está dada por

$$
N\left(>D_{0,04}\right)=6,4 \times 10^{5}\left(\frac{1 \mathrm{~km}}{D_{0,04}}\right)^{2,0 \pm 0,3}
$$

para $4.4 \leq D_{0,04} \leq 40 \mathrm{~km}, \mathrm{y}$

$$
N\left(>D_{0,04}\right)=1,8 \times 10^{10}\left(\frac{1 \mathrm{~km}}{D_{0,04}}\right)^{4,5 \pm 0,9}
$$

para $D_{0,04} \geq 84 \mathrm{~km}$. La Figura 6.1 a ha sido extraída del trabajo de Jewitt et al. (2000) y representa la distribución acumulada de Troyanos obtenida por estos autores, como función del tamaño y de la magnitud absoluta $\mathrm{H}$.

Algunos años más tarde, Yoshida \& Nakamura (2005) desarrollaron un estudio de la población de Troyanos jovianos, haciendo uso de observaciones realizadas con el Telescopio Subaru de $8.2 \mathrm{~m}$ en Mauna Kea, Hawaii. Estos autores detectaron 51 Troyanos jovianos en el punto $L_{4}$, con tamaños en el rango de $0.7 \lesssim D_{0,04} \lesssim 12.3 \mathrm{~km}$. A partir de estos datos, Yoshida \& Nakamura (2005) analizaron la distribución de tamaños de los asteroides Troyanos jovianos del punto $L_{4}$ para $2 \leq D_{0,04} \leq 10 \mathrm{~km}$. Para este rango entero, los autores derivaron un valor para la pendiente media de la distribución de tamaños acumulada de $1.9 \pm 0.1$, el 


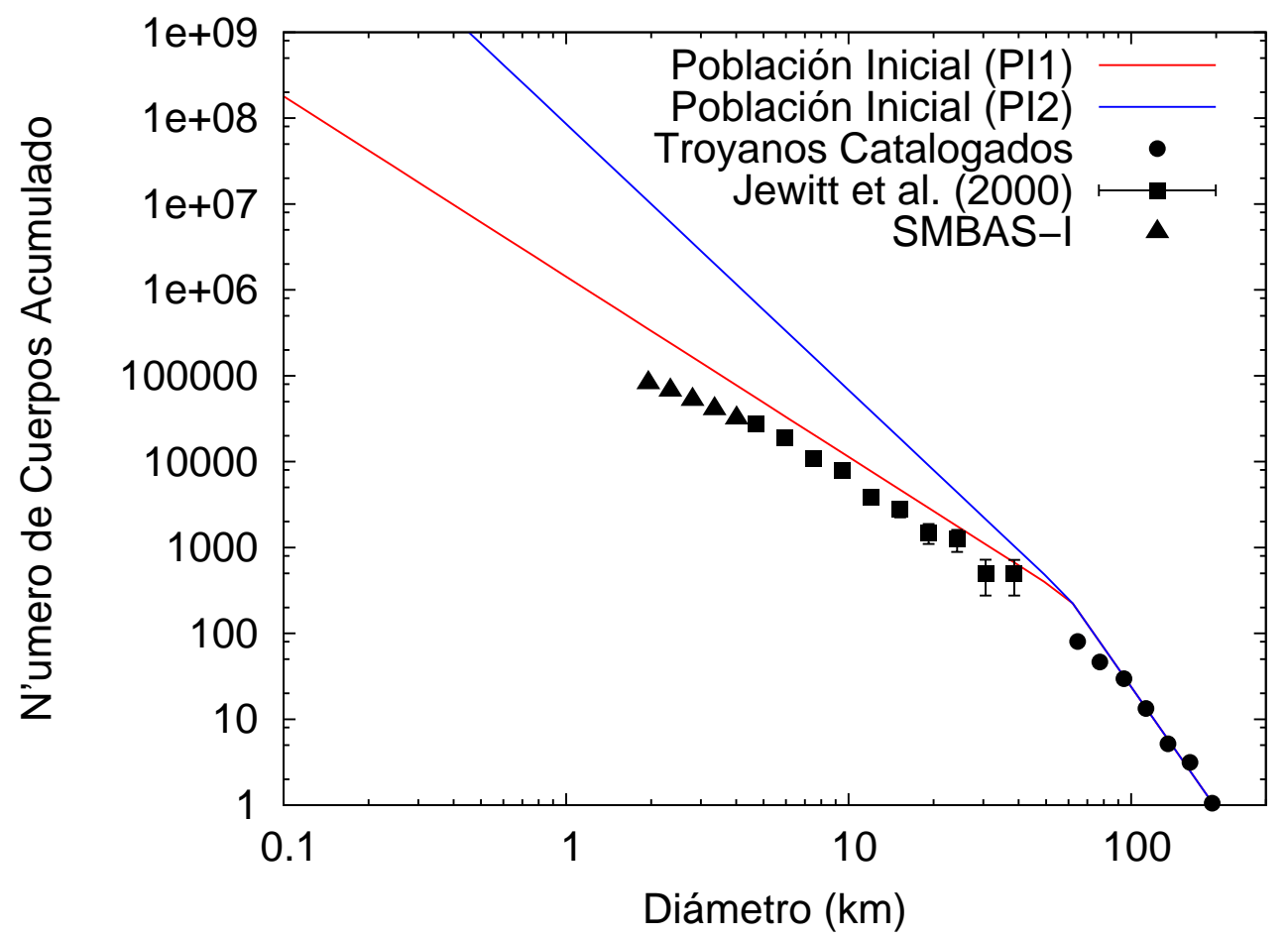

Figura 6.2. Poblaciones iniciales del modelo. Los datos derivados a partir de Troyanos catalogados, la distribución estimada por Jewitt et al. (2000), y los resultados derivados por Yoshida \& Nakamura (2005) en el Subaru Main Belt Asteroid Survey (SMBAS-I) también son incluidos.

cual es consistente con aquel previamente estimado por Jewitt et al. (2000) para los Troyanos jovianos de $L_{4}$ con $4.4 \leq D_{0,04} \leq 40 \mathrm{~km}$ (Ecuación 6.3). Sin embargo, Yoshida \& Nakamura (2005) notaron que la distribución de tamaños de los Troyanos de $L_{4}$ muestra un suave quiebre a un diámetro $D_{0,04} \sim 5 \mathrm{~km}$. A partir de esto, ellos derivaron pendientes medias para la distribución de tamaños acumulada de $1.28 \pm 0.11$ para $2 \leq D_{0,04} \leq 5 \mathrm{~km}$, y $2.39 \pm 0.10$ para $5 \leq D_{0,04} \leq 10 \mathrm{~km}$. La Figura $6.1 \mathrm{~b}$ ha sido extraída del trabajo de Yoshida \& Nakamura (2005) y representa la distribución acumulada de Troyanos obtenida por estos autores, como función del tamaño y de la magnitud absoluta $H$.

Las simulaciones numéricas desarrolladas por Davis \& Weidenschilling (1981) y Marzari et al. (1997) indican que las asteroides Troyanos jovianos de mayor tamaño estarían inalterados por impactos catastróficos desde las etapas más primitivas de la historia del Sistema Solar. Binzel \& Sauter (1992) reportaron observaciones de curvas de luz para asteroides Troyanos indicando que sólo aquellos con $D_{0,04} \gtrsim 90 \mathrm{~km}$ han sido capaces de retener sus formas iniciales después de $4.5 \times 10^{9}$ años de evolución colisional. A partir de esto, dichos autores sugirieron que un diámetro $D \sim 90 \mathrm{~km}$ podría representar una transición entre una población primordial y fragmentos colisionales producidos por cuerpos de mayor tamaño. Por otra parte, Jewitt et al. (2000) encontraron un diámetro crítico $D \sim 60 \mathrm{~km}$ para la distribución de tamaños de los Troyanos jovianos de $L_{4}$, el cual puede ser visto igualando las Ecuaciones 6.3 y 6.4. Jewitt et al. (2000) concluyeron que, ya que el diámetro de transición estimado por Binzel \& Sauter (1992) es incierto dentro de un factor 2, la distribución de tamaños y la distribución 
de amplitudes de las curvas de luz de los asteroides Troyanos indican que una transición entre una población primordial y fragmentos colisionales ocurre a un diámetro $D \sim 60-80 \mathrm{~km}$.

De este modo, para $D>60 \mathrm{~km}$, la distribución de tamaños acumulada inicial usada en nuestro modelo tiene una pendiente con una valor cercano a aquella determinada por los Troyanos observados en este rango de tamaños. Por otra parte, para $D \leq 60 \mathrm{~km}$, asignamos una pendiente $p$ a la distribución de tamaños acumulada inicial con el objetivo de reproducir una dada masa. De acuerdo con esto, la población acumulada inicial usada en nuestro algoritmo para estudiar la evolución de los Troyanos de $L_{4}$ es definida a partir de las siguientes expresiones:

$$
\begin{array}{lll}
N(>D)=C\left(\frac{1 \mathrm{~km}}{D}\right)^{p} & \text { para } & D \leq 60 \mathrm{~km}, \\
N(>D)=6 \times 10^{10}\left(\frac{1 \mathrm{~km}}{D}\right)^{4,7} & \text { para } & D>60 \mathrm{~km},
\end{array}
$$

donde $C=6 \times 10^{10}(60)^{-4,7}(60)^{p}$, por continuidad para $D=60 \mathrm{~km}$. Para testear la dependencia de nuestras simulaciones con la distribución de tamaños inicial, usamos la Ecuación 6.5 y definimos dos poblaciones iniciales diferentes, que se presentan a continuación:

- PI1: la cual asume un valor de $p=2.1$ y con esto una masa inicial de $\sim 1.2$ veces la masa actual de los Troyanos de $L_{4}, \mathrm{y}$

- PI2: la cual asume un valor de $p=3.1$, y con esto una masa inicial de $\sim 108$ veces la masa actual de los Troyanos de $L_{4}$.

La masa actual de Troyanos jovianos de $L_{4}$ fue estimada por Jewitt et al. (2000) y tiene un valor de $5 \times 10^{23} \mathrm{~g}$. La Figura 6.2 muestra las poblaciones iniciales PI1 y PI2 usadas en nuestro modelo, junto con los datos derivados a partir de Troyanos catalogados, la distribución estimada por Jewitt et al. (2000), y los resultados derivados por Yoshida \& Nakamura (2005) en el Subaru Main Belt Asteroid Survey (SMBAS-I).

En la Sección 6.6, discutimos la dependencia de nuestros resultados sobre la población inicial del modelo.

\subsection{Tratamiento Dinámico}

La Figura 6.3 muestra la distribución de 1155 Troyanos jovianos asociados al punto $L_{4}$, extraídos de la base de datos del $\mathrm{MPC}^{1}$ (Minor Planet Center), con respecto al semieje mayor $a$, excentricidad $e$, e inclinación $i$. Estos gráficos indican que todos los Troyanos de Júpiter observados en $L_{4}$ presentan valores de $a, e$, e $i$ entre 4.7 y 5.7 UA, 0 y 0.3, y 0 y $60^{\circ}$, respectivamente. De aquí en más, estos serán los límites del enjambre $L_{4}$ en semieje mayor, excentricidad e inclinación con los cuales vamos a desarrollar nuestra tarea.

Dado que la muestra de Troyanos jovianos con la cual contamos resulta ser estadísticamente significativa, es posible asumir que las posiciones ocupadas por estos objetos en los planos $(a, e)$ y $(a, i)$ representan zonas estables de la resonancia, y partir de esto, definir un

\footnotetext{
${ }^{1}$ http://www.cfa.harvard.edu/iau/lists/JupiterTrojans.html
} 

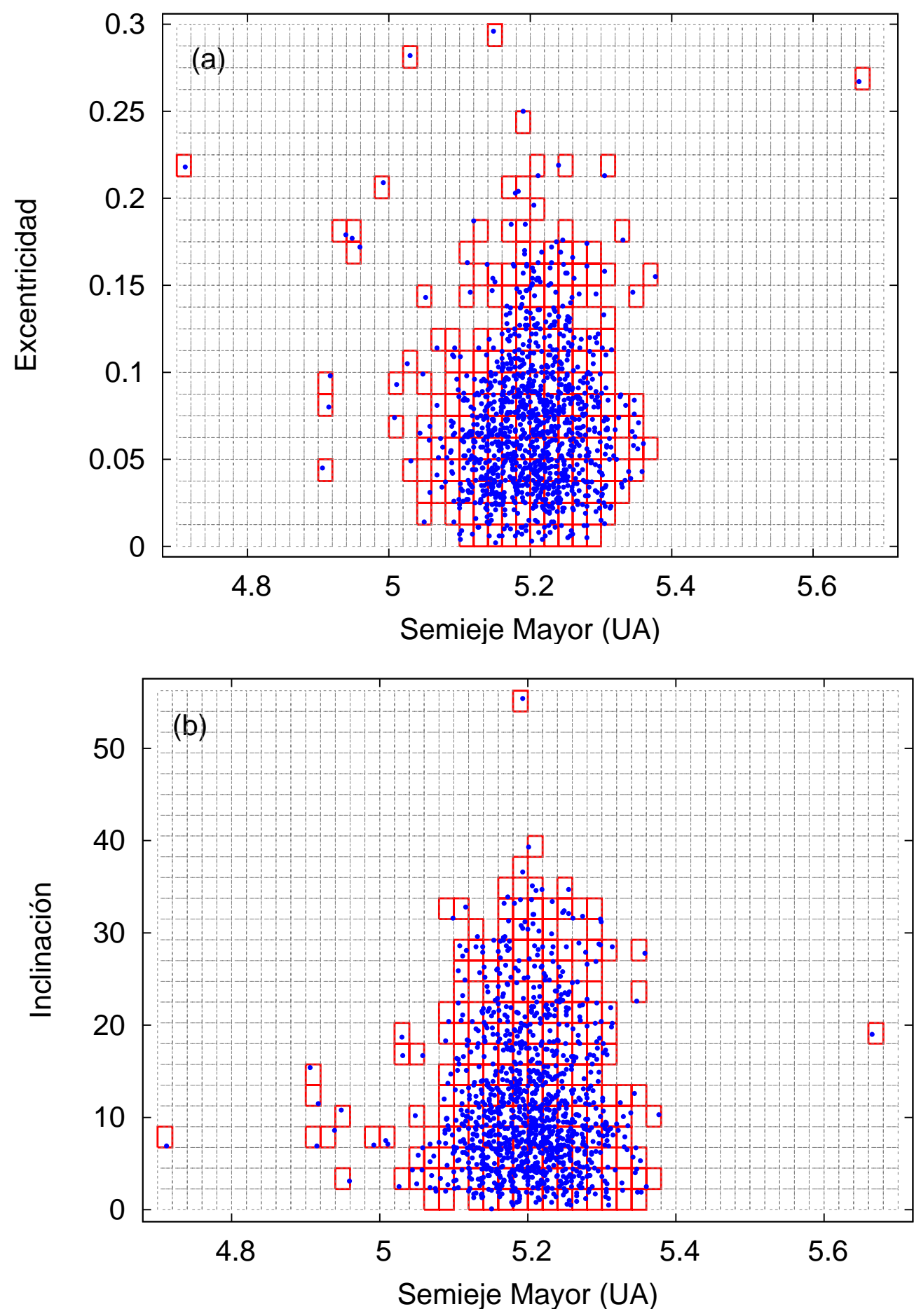

Figura 6.3. Distribución de los Troyanos jovianos de $L_{4}$ con respecto al semieje mayor, excentricidad e inclinación. Los cuadrados rojos y grises representan los nichos de estabilidad e inestabilidad usados en nuestras simulaciones, respectivamente. (Datos obtenidos de http://www.cfa.harvard.edu/iau/lists/JupiterTrojans.html) 


\begin{tabular}{ccc}
\hline & $\left\langle\boldsymbol{P}_{\mathrm{i}}\right\rangle$ & $\langle\boldsymbol{V}\rangle$ \\
$\times \mathbf{1 0}^{\mathbf{- 1 8}} \mathbf{k m}^{\mathbf{2}} \mathbf{\mathbf { a n n o } ^ { - 1 }}$ & $\mathbf{k m ~ s}^{-\mathbf{1}}$ \\
\hline $\boldsymbol{L}_{\mathbf{4}}$ & $7.79 \pm 0.67$ & 4.66 \\
\hline $\boldsymbol{L}_{\mathbf{5}}$ & $6.68 \pm 0.18$ & 4.51 \\
\hline
\end{tabular}

Tabla 6.1. Valores medios de la probabilidad intrínseca de colisión $\left\langle P_{\mathrm{i}}\right\rangle$ y la velocidad de impacto $\langle V\rangle$ derivados por Dell'Oro et al. (1998) para los dos enjambres Troyanos jovianos $L_{4}$ y $L_{5}$, a partir de una muestra de 223 objetos.

conjunto de nichos de estabilidad e inestabilidad dentro de los límites de la misma. En efecto, tales regiones son construidas asumiendo anchos de $0.02,0.0125$ y $2.25^{\circ}$ en semieje mayor, excentricidad e inclinación, respectivamente. Los nichos de estabilidad e inestabilidad generados a partir de este procedimiento son ilustrados en la Figura 6.3 como cuadrados rojos y grises, respectivamente.

Otra importante cuestión concerniente al comportamiento dinámico de los asteroides Troyanos jovianos es su amplitud de libración. De acuerdo a lo discutido en la Sección 3.1.3, Marzari et al $(2002,2003)$ sugirieron que las amplitudes de libración de estos objetos se encuentran en el rango que va desde $0.6^{\circ}$ a $88.7^{\circ}$, con un valor medio de $32.7^{\circ}$, para el punto $L_{4}$.

La Sección 6.5 describe cómo los nichos de estabilidad e inestabilidad mostrados en la Figura 6.3 son incluidos en nuestro algoritmo numérico con el objetivo de modelar el tratamiento dinámico del código. En la misma sección, también analizamos cómo la amplitud media de libración de estos objetos es usada para determinar el destino final de los fragmentos colisionales. Por otra parte, en la Sección 6.6.5 discutimos la sensibilidad de nuestros resultados a la forma en la cual aquellos nichos fueron construidos, así como también la dependencia de nuestras simulaciones sobre la distribución inicial de elementos orbitales de la población.

\subsection{Parámetros Colisionales y Relación con Otras Poblacio- nes}

\subsubsection{Probabilidad Intrínseca de Colisión y Velocidad de Impacto}

Para llevar a cabo el tratamiento colisional del modelo, adoptamos los valores medios de la probabilidad intrínseca de colisión $\left\langle P_{\mathrm{i}}\right\rangle$ y la velocidad de impacto $\langle V\rangle$ derivados por Dell'Oro et al. (1998), usando el algoritmo matemático desarrollado por Dell'Oro \& Paolicchi (1998), sobre una muestra de 223 Troyanos. Este método estadístico computa los valores de $\left\langle P_{\mathrm{i}}\right\rangle$ y $\langle V\rangle$ para los dos enjambres Troyanos jovianos $L_{4}$ y $L_{5}$ sobre una escala de tiempo de $10^{6}$ años, teniendo en cuenta la conexión dinámica entre las variables angulares orbitales de los Troyanos y Júpiter debido a la resonancia 1:1. Sobre dicha escala de tiempo, el efecto de la frecuencia secular $g_{5}-g_{6}$ llega a ser importante, afectando fuertemente el semieje mayor, la excentricidad y la amplitud de libración de todos los asteroides Troyanos. Por esta razón, la evolución en el tiempo de $\left\langle P_{\mathrm{i}}\right\rangle$ muestra grandes oscilaciones, mientras que el comportamiento de $\langle V\rangle$ es algo más complicado debido a las variaciones en la inclinación de estos objetos. Los valores medios de la la probabilidad intrínseca de colisión y la velocidad de impacto derivados 


\begin{tabular}{|c|c|c|}
\hline Poblaciones & $\begin{array}{c}\left\langle P_{\mathrm{i}}\right\rangle \\
\times 10^{-18} \mathrm{~km}^{-2} \mathrm{año}^{-1}\end{array}$ & Referencia \\
\hline $\operatorname{Troyanos}_{L_{4}}$-Hildas & $0.24 \pm 0.06$ & Dahlgren (1998) \\
\hline $\operatorname{Troyanos}_{L_{4}}$-Hildas & $0.27 \pm 0.01$ & Dell'Oro et al. (2001) \\
\hline $\operatorname{Troyanos}_{L_{4}}-\mathrm{JFC}$ & $0.33 \pm 0.01$ & Dell'Oro et al. (2001) \\
\hline
\end{tabular}

Tabla 6.2. Valores medios de la probabilidad intrínseca de colisión $\left\langle P_{\mathrm{i}}\right\rangle$ entre Troyanos jovianos de $L_{4}$ e Hildas y Cometas de la Familia de Júpiter (JFC).

por Dell'Oro et al. (1998) para los dos enjambres Troyanos jovianos son mostrados en la Tabla 6.1.

\subsubsection{Relaciones con Hildas y Cometas de la Familia de Júpiter}

Más allá de las mutuas colisiones, los Troyanos jovianos también pueden tener encuentros con asteroides Hildas y Cometas de la Familia de Júpiter. Para entender si tales poblaciones contribuyen a la evolución colisional de los Troyanos, es necesario analizar los siguientes puntos:

1. Las probabilidades intrínsecas de colisión entre Troyanos e Hildas, y entre Troyanos y Cometas de la Familia de Júpiter.

2. El número total estimado de asteroides Hildas y Cometas de la Familia de Júpiter capaces de impactar la población de Troyanos.

Dahlgren (1998) y Dell'Oro et al. (2001) estimaron la probabilidad intrínseca de colisión $\left\langle P_{\mathrm{i}}\right\rangle_{\mathrm{T}-\mathrm{H}}$ entre Troyanos e Hildas y encontraron que el valor de $\left\langle P_{\mathrm{i}}\right\rangle_{\mathrm{T}-\mathrm{H}}$ es $\sim 30$ veces menor que $\left\langle P_{\mathrm{i}}\right\rangle_{\mathrm{T}-\mathrm{T}}$ para colisiones entre asteroides Troyanos de $L_{4}$ (Tabla 6.2). Por otra parte, Brunini et al. (2003) determinaron que el número total de Hildas con un radio $R>1 \mathrm{~km}$ es, a lo sumo, igual a 25000 , lo cual es aproximadamente el $16 \%$ de la población estimada por Jewitt et al. (2000) en el enjambre Troyano joviano $L_{4}$ (Ecuación 6.3).

De la misma forma, Dell'Oro et al. (2001) computaron la probabilidad intrínseca de colisión $\left\langle P_{\mathrm{i}}\right\rangle_{\mathrm{T}-\mathrm{JFC}}$ entre Troyanos de $L_{4}$ y Cometas de la Familia de Júpiter y determinaron que el valor de $\left\langle P_{\mathrm{i}}\right\rangle_{\mathrm{T}-\mathrm{JFC}}$ es $\sim 24$ veces menor que $\left\langle P_{\mathrm{i}}\right\rangle_{\mathrm{T}-\mathrm{T}}$ para colisiones entre asteroides Troyanos de $L_{4}$ (Tabla 6.2). Además, Fernández et al. (1999) indicaron que el número total de Cometas de la Familia de Júpiter con radios $R>0.7 \mathrm{~km}$ es de algunos miles a casi $10^{4}$, el cual es de uno a dos órdenes de magnitud más pequeño que el número total de Troyanos de $L_{4}$ derivado por Jewitt et al. (2000) (Ecuación 6.3).

A partir de este análisis, inferimos que la contribución de los asteroides Hildas y los Cometas de la Familia de Júpiter a la evolución colisional de los asteroides Troyanos resulta ser despreciable.

\subsubsection{Leyes de Impacto}

De acuerdo a los argumentos presentados en la Sección 3.3, decidimos seleccionar como parámetros de entrada de nuestro modelo colisional una combinación de leyes $Q_{\mathrm{S}}$ y funciones 

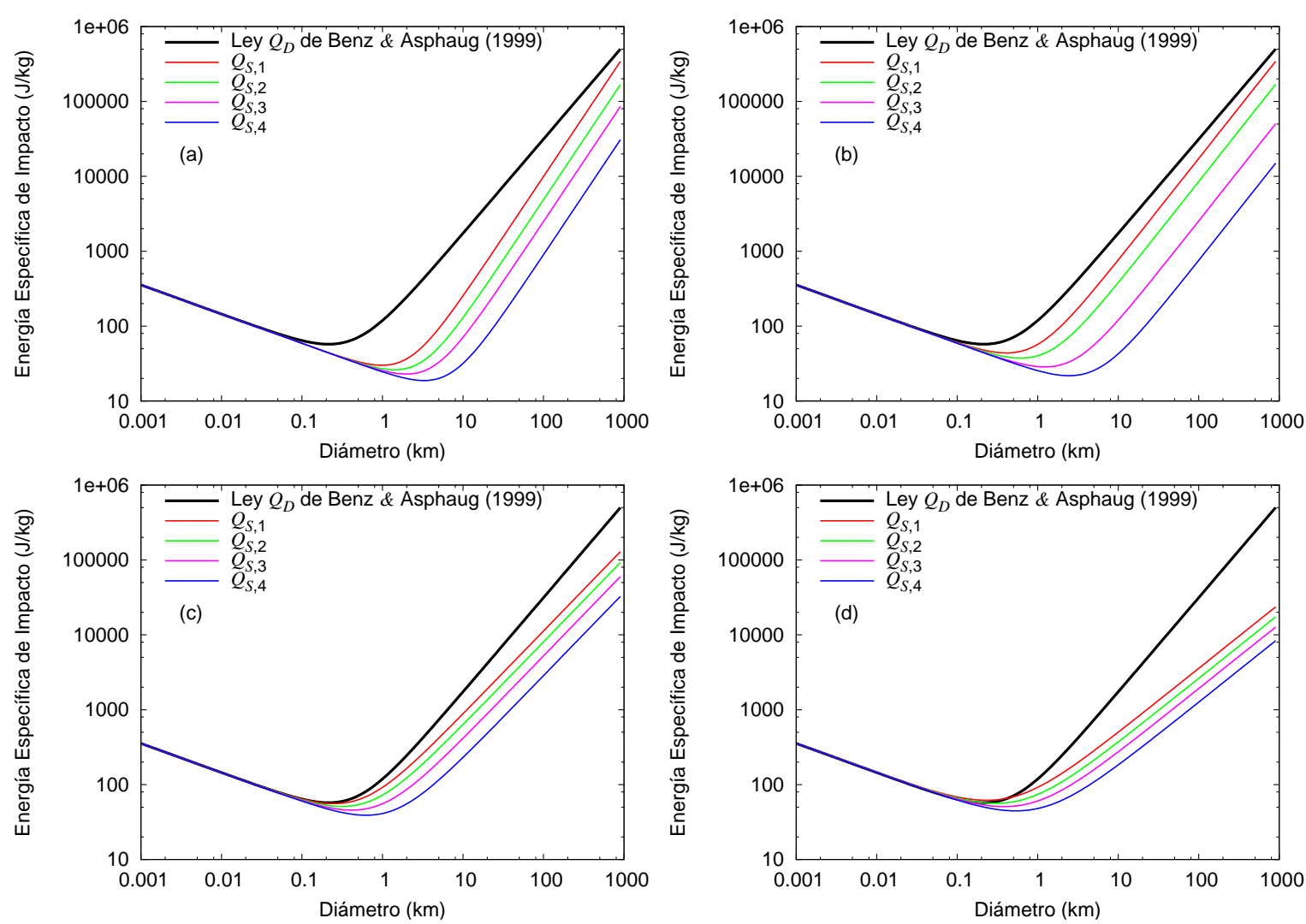

Figura 6.4. Leyes de Impacto. Las líneas de colores representan las leyes $Q_{\mathrm{S}}$ usadas en nuestras simulaciones. Desde (a) hasta (d), las curvas $Q_{\mathrm{S}}$ muestran un decrecimiento en las pendientes para $D \gtrsim 1 \mathrm{~km}$, cubriendo todas las posibilidades desde pequeños a grandes gaps entre $Q_{\mathrm{S}}$ y la ley $Q_{\mathrm{D}}$ de Benz \& Asphaug (1999) para cuerpos helados a $3 \mathrm{~km} \mathrm{~s}^{-1}$, la cual es ilustrada a través de la curva de color negro.

$f_{\text {ke }}$ que produzcan la ley $Q_{\mathrm{D}}$ derivada por Benz \& Asphaug (1999) a partir de estudios hidrodinámicos, para cuerpos de hielo a velocidades de $3 \mathrm{~km} \mathrm{~s}^{-1}$. Esta ley $Q_{\mathrm{D}}$ es ilustrada en la Figura 6.4 a través de una curva negra y puede ser calculada a partir de una expresión de la forma

$$
Q_{\mathrm{D}}=C_{1} D^{-\lambda_{1}}\left(1+\left(C_{2} D\right)^{\lambda_{2}}\right)
$$

donde $C_{1}, C_{2}, \lambda_{1}$, y $\lambda_{2}$ son coeficientes cuyos valores son $24,2.3,0.39$ y 1.65 , respectivamente.

Para analizar la dependencia de nuestras simulaciones numéricas con la energía específica de fragmentación $Q_{\mathrm{S}}$, nuestro modelo adopta una numerosa familia de curvas $Q_{\mathrm{S}}$ testeando diferentes pendientes para $D \gtrsim 1 \mathrm{~km}$ y cubriendo todas las posibilidades desde pequeños a grandes gaps entre $Q_{\mathrm{S}}$ y la ley $Q_{\mathrm{D}}$ de Benz \& Asphaug (1999). En efecto, las leyes $Q_{\mathrm{S}}$ usadas en nuestras simulaciones son ilustradas en la Figura 6.4 a través de las curvas de colores, y pueden ser representadas a partir de expresiones de la forma

$$
Q_{\mathrm{S}}=C_{1}^{\prime} D^{-\lambda_{1}^{\prime}}\left(1+\left(C_{2}^{\prime} D\right)^{\lambda_{2}^{\prime}}\right),
$$

donde $C_{1}^{\prime}, C_{2}^{\prime}, \lambda_{1}^{\prime}$, y $\lambda_{2}^{\prime}$ son coeficientes constantes para cada curva. Para cuerpos pequeños, con diámetros $D \lesssim 1 \mathrm{~km}$, la energía de ligadura gravitacional es despreciable, por lo cual $Q_{\mathrm{S}}$ 
y $Q_{\mathrm{D}}$ tienen el mismo valor en el rango de tamaños mencionado. De este modo, $C_{1}^{\prime}=C_{1}$ y $\lambda_{1}^{\prime}=\lambda_{1}$ para todas las leyes $Q_{\mathrm{S}}$, ya que tales coeficientes dominan el comportamiento de las curvas para pequeños tamaños. Por otra parte, los coeficientes $\lambda_{2}^{\prime}$ y $C_{2}^{\prime}$ determinan la pendiente y la magnitud de cada ley $Q_{\mathrm{S}}$ para diámetros $D \gtrsim 1 \mathrm{~km}$, respectivamente. Los valores de $\lambda_{2}^{\prime}$ usados en las Figuras $6.4 \mathrm{a}$, b, c y d son 2, 1.75, 1.5 y 1.25, respectivamente. En cuanto a $C_{2}^{\prime}$, este coeficiente toma valores en el rango de 0.5 a 0.15 , de 1.2 a 0.2 , de 2 a 0.8 , y de 2.3 a 1 para las leyes $Q_{\mathrm{S}}$ mostradas en las Figuras $6.4 \mathrm{a}$, b, c y d, respectivamente.

Para modelar $f_{\text {ke }}$ seguimos los lineamientos propuestos en la Sección 4.4. A partir de esto, el parámetro $f_{\text {ke }}$ adopta una expresión de la forma

$$
f_{\mathrm{ke}}=f_{\mathrm{ke}_{0}}\left(\frac{D}{1000 \mathrm{~km}}\right)^{\gamma} .
$$

Los valores de $f_{\mathrm{ke}_{0}} \mathrm{y} \gamma$ usados en nuestras simulaciones son 0.35 y 0.7 , respectivamente, los cuales son consistentes con aquellos discutidos por Davis et al. (1989). En efecto, a partir de la combinación de cualquiera de las leyes $Q_{\mathrm{S}}$ mostradas en la Figura 6.4 y $f_{\mathrm{ke}}$, con $f_{\mathrm{ke}_{0}}=0.35$ y $\gamma=0.7$, la ley $Q_{\mathrm{D}}$ derivada por Benz \& Asphaug (1999) es obtenida con buena precisión.

\subsection{Modelo Numérico de Evolución Colisional y Dinámica}

En esta sección presentamos el modelo dinámico y colisional usado para estudiar la evolución de los asteroides Troyanos jovianos asociados al punto $L_{4}$.

Al igual que lo indicado en la Sección 5.6 y de acuerdo a lo sugerido por Campo Bagatin et al. (1994a) y Campo Bagatin (1998), nuestro algoritmo no evoluciona en el tiempo los primeros 60 bines de tamaño, cuyos diámetros centrales van desde $10^{-10}$ a $10^{-4} \mathrm{~km}$. Para los Troyanos jovianos de $L_{4}$, esta parte de la población es usada únicamente a modo de proyectiles para computar tasas de impacto con cuerpos más grandes, y su distribución de tamaños es determinada en cada paso de tiempo, extrapolando la pendiente de la distribución asociada a los 10 bines de tamaño siguientes.

El modelo numérico usado en este estudio es equivalente a aquel descripto en la Sección 5.6 y utilizado para analizar la evolución simultánea del Cinturón Principal de asteroides y la población de NEAs. En este caso, el código resulta ser más simple ya que sólo se involucra a los Troyanos jovianos de $L_{4}$ como única población del modelo.

Supongamos que analizamos una colisión entre dos Troyanos $o$ y $o^{\prime}$ con diámetros $D_{o}$ y $D_{o^{\prime}}$, respectivamente. Las distribuciones de fragmentos y velocidades, y la velocidad de escape del sistema de cuerpos colisionantes, son calculadas con el modelo colisional descripto en el Capítulo 4 y de la forma detallada en la Sección 5.6. Para determinar el destino final de los fragmentos que escapan del sistema de cuerpos colisionantes, proponemos un mecanismo simple en el cual se tienen en cuenta las características orbitales de los Troyanos jovianos de $L_{4}$ (Sección 6.3). Como paso inicial a realizar en cada intervalo de tiempo, una órbita característica es generada al azar para cada colisión entre Troyanos de diámetros $D_{o}$ y $D_{o^{\prime}}$ en el punto $L_{4}$. Para esto, usamos el método de aceptación-rechazo desarrollado por John von Neumann (Knuth 1981). A partir de las Figuras 6.3a y b, construimos nichos tridimensionales dentro de los límites de $L_{4}$ con anchos de $0.02 \mathrm{UA}, 0.0125$ y $2.25^{\circ}$ en semieje mayor $a$, excentricidad $e$ e inclinación $i$, respectivamente. En cada una de estas zonas, calculamos la 
fracción de Troyanos $f(a, e, i)=N_{(a, e, i)}^{\text {Troyanos }} / N_{\text {Total }}^{\text {Troyanos }}$, donde $N_{\text {Total }}^{\text {Troyanos }}$ representa el número total de Troyanos de la muestra, el cual es igual a 1155 (Sección 6.3). Este procedimiento nos permite definir una función $f$ de $a, e$ e $i$ cuyo valor máximo resulta ser $\sim 0.0065$. La técnica propuesta por von Neumann indica que si un conjunto de números $a^{*}, e^{*}$ e $i^{*}$ es seleccionado aleatoriamente del dominio de la función $f$ (esto es, $a^{*}, e^{*}$ e $i^{*}$ entre 4.7 y $5.7 \mathrm{UA}, 0$ y 0.3 y 0 y $60^{\circ}$, respectivamente), y otro conjunto de números $f^{*}$ es tomado al azar desde el rango de tal función (esto es, $f^{*}$ entre 0 y 0.0065$)$, entonces la condición $f^{*} \leq f\left(a^{*}, e^{*}, i^{*}\right)$ generará una distribución para $\left(a^{*}, e^{*}, i^{*}\right)$ cuya densidad es $f\left(a^{*}, e^{*}, i^{*}\right) d a^{*} d e^{*} d i^{*}$. Tales valores de $\left(a^{*}, e^{*}, i^{*}\right)$ serán aceptados como posibles condiciones iniciales para el semieje mayor, excentricidad e inclinación de los Troyanos de $L_{4}$, en acuerdo con los datos observacionales. Vale la pena notar que en las resonancias de movimientos medios, la evolución de $a, e$, e $i$ es acoplada. Sin embargo, las mismas son tratadas aquí como variables no correlacionadas. Destacamos que un tratamiento más riguroso sería muy dificultoso, y creemos que los resultados no serían demasiado diferentes a aquellos derivados en este análisis. Finalmente, dada la longitud del nodo ascendente $\Omega$, el argumento del perihelio $\omega$ y la anomalía media $M$ entre 0 y $360^{\circ}$, una órbita puede ser asignada y a partir de esto, un par posición-velocidad puede ser derivado para cada uno de los Troyanos colisionantes.

Para determinar el destino final de los fragmentos que escapan, es necesario calcular cuáles son sus elementos orbitales una vez que ellos son eyectados con una dada velocidad respecto del cuerpo que los originó. Inmediatamente antes de la colisión, asumimos que la posición y velocidad baricéntricas de los fragmentos son iguales a aquellas asociadas a su cuerpo precursor. Inmediatamente después de la colisión, consideramos que la posición baricéntrica de los fragmentos es equivalente a aquella asociada a su cuerpo precursor, mientras que la velocidad relativa de los mismos con respecto a este último es igualmente particionada entre las tres componentes cartesianas. Una vez que obtenemos la posición y velocidad baricéntricas de los fragmentos posteriores al impacto, es posible calcular sus elementos orbitales y sus destinos finales. Para hacer esto, usamos los siguientes criterios:

1. Los fragmentos permanecen en el enjambre joviano $L_{4}$ si las combinaciones de valores $(a, e)$ y $(a, i)$ están asociadas con alguno de los nichos de estabilidad mostrados en las Figuras 6.3a y b, respectivamente, y el valor absoluto de la diferencia entre sus longitudes medias inicial y final es menor que la amplitud media de libración, cuyo valor es $\sim 30^{\circ}$ (Sección 6.3).

2. Por otra parte, los fragmentos son eyectados desde el enjambre joviano $L_{4}$, dejando de participar de la evolución colisional, si se satisface cualquiera de las siguientes condiciones:

- excentricidad $e \geq 1$,

- excentricidad $e<1$, con valores $(a, e, i)$ que exceden los límites del enjambre $L_{4}$ (Sección 6.3),

- excentricidad $e<1$, con valores $(a, e)$ y $(a, i)$ asociados con alguno de los nichos de inestabilidad mostrados en las Figuras $6.3 \mathrm{a}$ y b, respectivamente,

- excentricidad $e<1$, con valores de $(a, e)$ y $(a, i)$ asociados con alguno de los nichos de estabilidad mostrados en las Figuras $6.3 \mathrm{a}$ y b, respectivamente, pero con un valor absoluto para la diferencia entre las longitudes medias inicial y final mayor que la amplitud media de libración de $\sim 30^{\circ}$. 
Bajo estas circunstancias, los fragmentos tienen dos posibilidades: permanecer en la población de Troyanos de $L_{4}$ o escapar fuera de la resonancia. El número de fragmentos eyectados desde los cuerpos o y $o^{\prime}$ que permanecen en el enjambre $L_{4}$ es guardado en los vectores $F_{o}(k)$ y $F_{o^{\prime}}(k)$, respectivamente. El número de fragmentos que no permanecen en el enjambre $L_{4}$ no debe ser guardado en dichos vectores. Sin embargo, esta información también debe ser conservada, ya que la misma resulta ser relevante para computar la tasa de eyección colisional desde dicha región en cada paso de tiempo.

La frecuencia de colisión entre Troyanos o y $o^{\prime}$, con diámetros $D_{o}$ y $D_{o^{\prime}}$, respectivamente, puede ser calculada a partir de la siguiente expresión

$$
f_{o, o^{\prime}}=\frac{\left\langle P_{\mathrm{i}}\right\rangle}{4}\left(D_{o}+D_{o^{\prime}}\right)^{2}
$$

donde $\left\langle P_{\mathrm{i}}\right\rangle$ es la probabilidad intrínseca de colisión entre Troyanos de $L_{4}$ (Sección 6.4.1), y tiene unidades de $\mathrm{km}^{-2}$ año ${ }^{-1}$. Si $\Delta t$ representa un dado intervalo y $N_{o, o^{\prime}}$ es el número de pares de Troyanos con diámetros $D_{o}$ y $D_{o^{\prime}}$, es posible obtener el número total de colisiones entre dichos objetos durante $\Delta t$ a partir de la expresión

$$
N_{\mathrm{col}}=f_{o, o^{\prime}} N_{o, o^{\prime}} \Delta t
$$

Sean $N_{o}$ y $N_{o^{\prime}}$ el número de Troyanos de diámetro $D_{o}$ y $D_{o^{\prime}}$, respectivamente. Tal como se indicó en la Sección 5.6, si los cuerpos colisionantes pertenecen al mismo bin de tamaño, esto es, si $o=o^{\prime}$, entonces $N_{o}=N_{o^{\prime}}$ y por ende, el número de pares de Troyanos con diámetros $D_{o}$ y $D_{o^{\prime}}$ está dado por

$$
N_{o, o^{\prime}}=\frac{N_{o}\left(N_{o^{\prime}}-1\right)}{2} .
$$

Para todos los otros casos,

$$
N_{o, o^{\prime}}=N_{o} N_{o^{\prime}}
$$

A partir de esto, el número total de fragmentos asociados al bin de tamaño $k$ que permanecen en $L_{4}$ luego de ser eyectados de los cuerpos o como resultado de colisiones con cuerpos $o^{\prime}$, durante un intervalo $\Delta t$, está dado por $F_{o}(k) f_{o, o^{\prime}} N_{o, o^{\prime}} \Delta t$. Equivalentemente, el número total de fragmentos asociados al bin de tamaño $k$ que permanecen en $L_{4}$ luego de ser eyectados de los cuerpos $o^{\prime}$ como resultado de colisiones con cuerpos $o$, durante un intervalo $\Delta t$, está dado por $F_{o^{\prime}}(k) f_{o, o^{\prime}} N_{o, o^{\prime}} \Delta t$. Si tenemos en cuenta las interacciones entre todos los objetos de la población de Troyanos de $L_{4}$, es posible derivar la variación en el número de cuerpos asociados a un dado bin de tamaño $k$, en cada paso de tiempo. Dichas variaciones son referidas como $\Delta N_{k}$.

Para estudiar la evolución en el tiempo de la población de Troyanos de $L_{4}$, el paso de tiempo $\Delta t$ es calculado en una forma tal que el cambio relativo en el número de objetos en cualquier bin de tamaño sea siempre menor que el $1 \%$.

\subsection{Resultados}

Aquí, nosotros discutimos los resultados generales obtenidos a partir de una serie de simulaciones numéricas destinadas a estudiar la evolución colisional y dinámica de la población de Troyanos jovianos de $L_{4}$ sobre $4.5 \times 10^{9}$ años. 

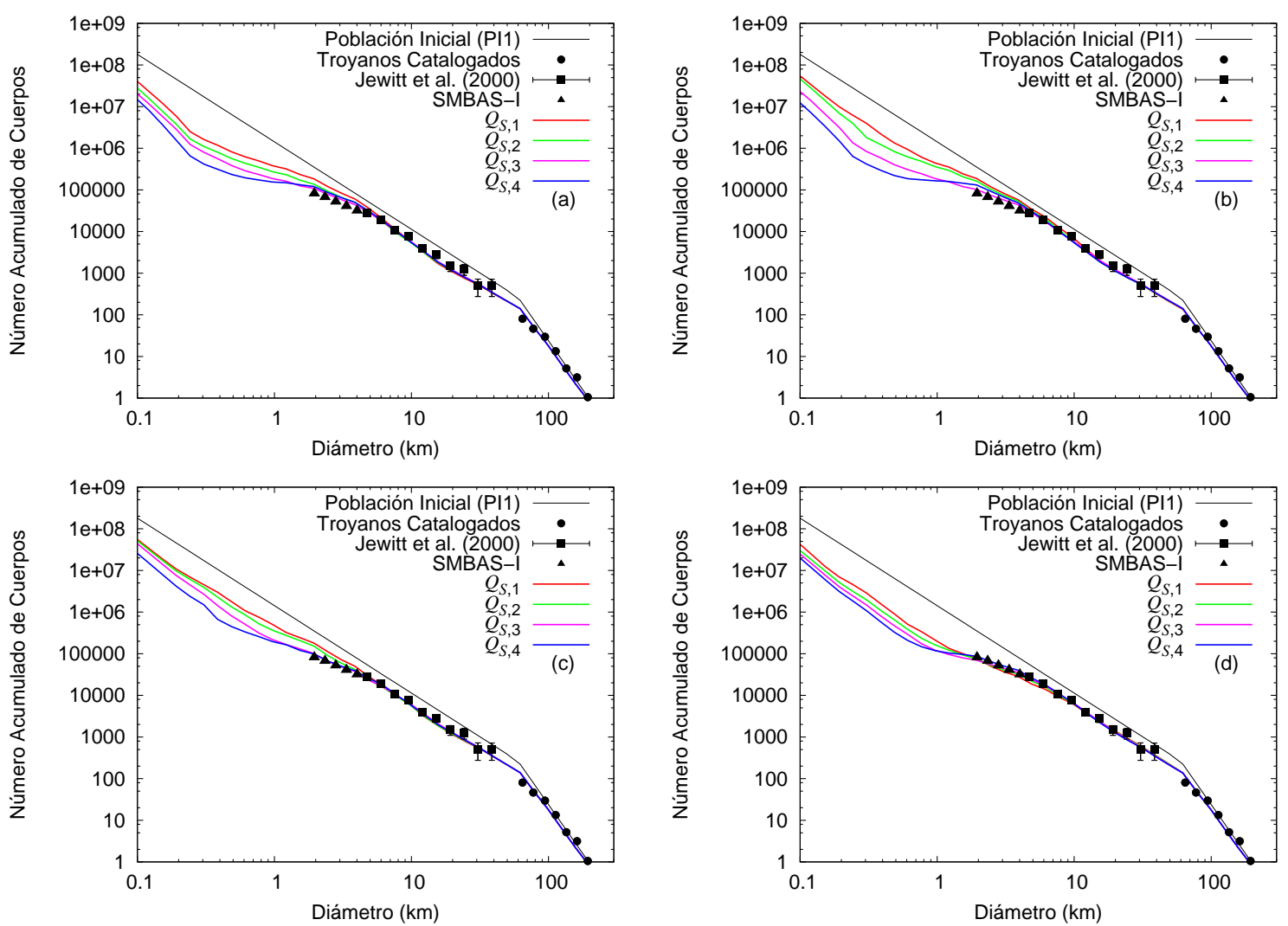

Figura 6.5. a, b, c y d muestran las distribuciones de tamaños acumuladas de los Troyanos de $L_{4}$ obtenidas a partir de las leyes $Q_{\mathrm{S}}$ presentadas en las Figuras $6.4 \mathrm{a}$, b, c y d, respectivamente. Notemos que este conjunto de simulaciones numéricas ha sido desarrollado haciendo uso de la población inicial PI1. En términos generales, las distribuciones de tamaños acumuladas de los Troyanos de $L_{4}$ obtenidas a partir de este análisis son consistentes con los datos observacionales. Nuestras simulaciones muestran ondas en las distribuciones de tamaños evolucionadas finales, las cuales resultan más evidentes para $D \lesssim 1 \mathrm{~km}$. Es posible observar que la amplitud de estas ondas es menor cuanto más chico es el gap entre las leyes $Q_{\mathrm{S}}$ y $Q_{\mathrm{D}}$.

\subsubsection{Distribución de Tamaños de los Troyanos de $L_{4}$}

Las Figuras 6.5a, b, c y d muestran las distribuciones de tamaños de los Troyanos de $L_{4}$ obtenidas a partir de la familia de curvas $Q_{\mathrm{S}}$ presentadas en las Figuras $6.4 \mathrm{a}$, b, c y d, respectivamente. Notemos que este conjunto de simulaciones numéricas ha sido desarrollado haciendo uso de la población inicial PI1. En términos generales, las distribuciones de tamaños acumuladas de los Troyanos de $L_{4}$ obtenidas a partir de este análisis son consistentes con los datos observacionales. Los resultados de nuestras simulaciones muestran ondas en las distribuciones de tamaños evolucionadas finales, las cuales se propagan desde diámetros $D$ de $\sim 0.1 \mathrm{a} \sim 60 \mathrm{~km}$. Para $D \lesssim 1 \mathrm{~km}$, donde la estructura de onda resulta ser más evidente, es posible observar que cuanto más chico es el gap entre las leyes $Q_{\mathrm{S}}$ y $Q_{\mathrm{D}}$, menor es la amplitud de la onda. Tal como indicamos en la Sección 3.3, esta estructura de onda se genera como consecuencia de una transición en las propiedades del material, la cual se produce para diámetros $D \sim 0.1-1 \mathrm{~km}$. 

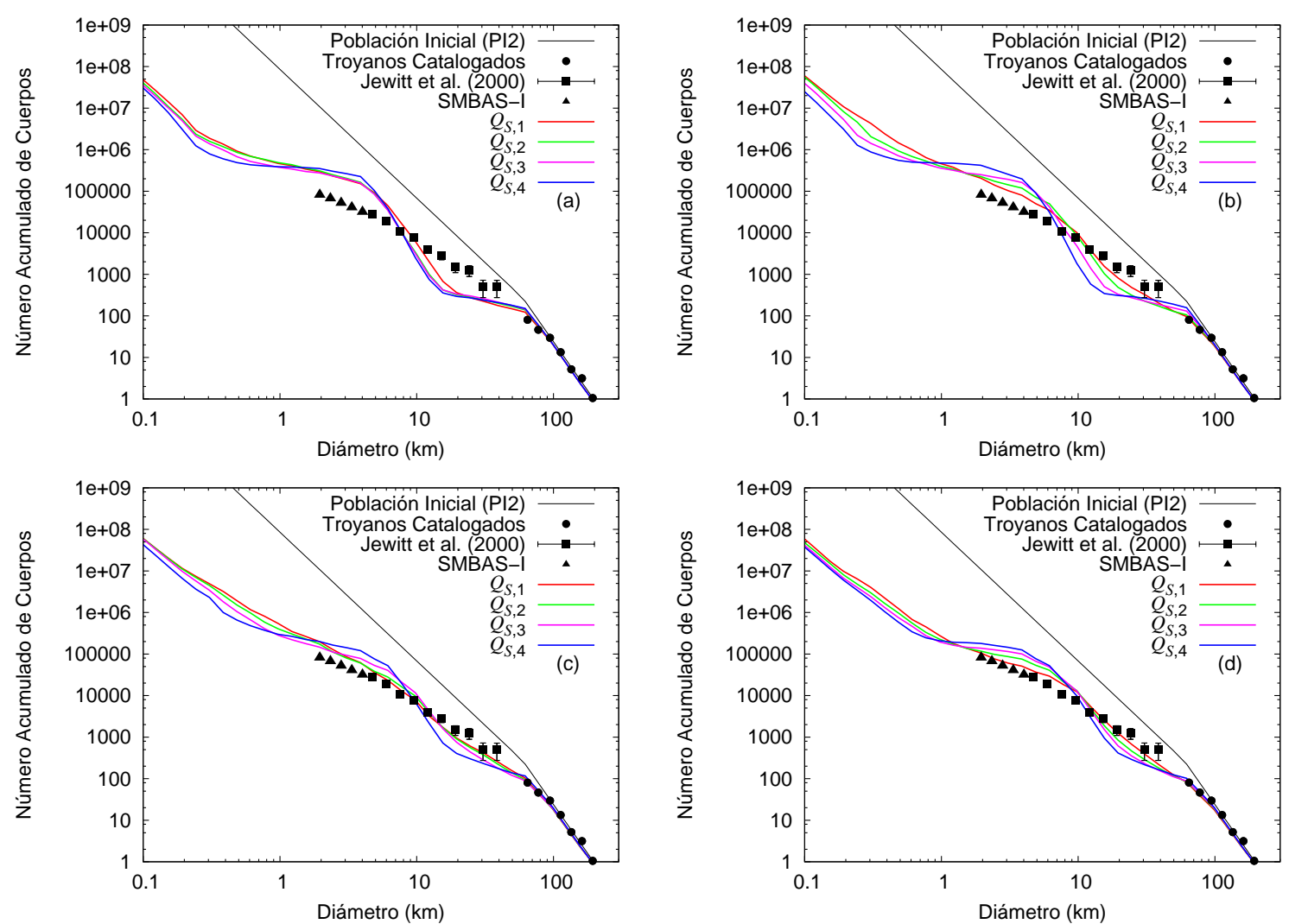

Figura 6.6. a, b, c y d muestran las distribuciones de tamaños acumuladas de los Troyanos de $L_{4}$ obtenidas a partir de las leyes $Q_{\mathrm{S}}$ presentadas en las Figuras $6.4 \mathrm{a}$, b, c y d, respectivamente. Notemos que este conjunto de simulaciones numéricas ha sido desarrollado haciendo uso de la población inicial PI2. En términos generales, la distribución de tamaños observada de los Troyanos de $L_{4}$ no es bien reproducida. En este caso, la estructura de onda resulta ser más evidente que en la Figura 6.5, observándose una menor amplitud cuanto más chico es el gap entre las leyes $Q_{\mathrm{S}}$ y $Q_{\mathrm{D}}$.

La Figura 6.6 muestra las distribuciones de tamaños acumuladas de los Troyanos de $L_{4}$ derivadas haciendo uso de la población inicial PI2. Notemos que los resultados representados en las Figuras 6.6a, b, c y d son obtenidos a partir de las leyes $Q_{\mathrm{S}}$ de las Figuras 6.4a, b, c y d, respectivamente. En términos generales, la distribución de tamaños observada de los Troyanos de $L_{4}$ no es bien reproducida, generándose ondas con amplitudes demasiado grandes. Como ya fue mencionado anteriormente, la estructura de onda muestra una menor amplitud cuanto más chico es el gap entre las leyes $Q_{\mathrm{S}}$ y $Q_{\mathrm{D}}$.

De acuerdo a lo observado en las Figuras 6.5 y 6.6, cuanto mayor es la masa de la población inicial, mayor resulta la amplitud de la onda generada en la distribución de tamaños evolucionada final. De este modo, concluimos que cuanto menor es la masa de la población inicial, mejor se reproduce la distribución de tamaños observada de los Troyanos jovianos de $L_{4}$.

Para todos nuestros experimentos numéricos, las posiciones de los picos y valles de la onda no cambian significativamente. En efecto, las Figuras 6.5 y 6.6 muestran que el primer valle se extiende desde diámetros $D$ de $\sim 0.1$ a $1 \mathrm{~km}$, produciendo un pico alrededor de los $5 \mathrm{~km}$, lo 
cual lleva a un segundo valle a un diámetro $D \sim 20 \mathrm{~km}$. Estos resultados nos permiten inferir que la forma general de la población evolucionada final está determinada principalmente por la energía específica de dispersión $Q_{\mathrm{D}}$ más que por la energía específica de fragmentación $Q_{\mathrm{S}}$, lo cual es consistente con aquello discutido por O'Brien \& Greenberg (2005).

Tal como hemos mencionado en los párrafos anteriores, los más grandes gaps entre las curvas $Q_{\mathrm{S}}$ y $Q_{\mathrm{D}}$ llevan a las más grandes amplitudes de onda en la distribución de tamaños evolucionada final. Con el objetivo de entender este comportamiento, llevamos a cabo dos simulaciones numéricas destinadas a analizar cómo el proceso de dispersión de fragmentos resultantes de eventos catastróficos y de craterización es afectado por la relación entre $Q_{\mathrm{S}}$ y $Q_{\mathrm{D}}$. Para hacer esto, seleccionamos las leyes $Q_{\mathrm{S}, 1}$ y $Q_{\mathrm{S}, 4}$ presentadas en la Figura 6.4b, las cuales muestran un gap chico y grande con la ley $Q_{\mathrm{D}}$ de Benz \& Asphaug (1999), respectivamente. Usando la ley $Q_{\mathrm{S}, 4}$ especificada, nuestra simulación muestra que el número de fragmentos dispersados con diámetros $D>0.1 \mathrm{~km}$, resultantes de eventos catastróficos y de craterización, es 55-75\% menor comparado con aquel derivado a partir de la simulación con el gap pequeño (Figura 6.7a). Además, más del $99 \%$ del total de los fragmentos dispersados con diámetros $D>0.1 \mathrm{~km}$ se ubica en el primer valle de la onda (esto es, en el rango de diámetros $0.1<D<1 \mathrm{~km}$ ) para ambas simulaciones. En efecto, el número de fragmentos dispersados con $D>1 \mathrm{~km}$ resulta ser algunos órdenes de magnitud menor que el número total de fragmentos dispersados con $D>0.1 \mathrm{~km}$ (Figuras $6.7 \mathrm{a}$ y b). De este modo, el número de fragmentos capaces de resuministrar los bines de tamaños en el rango de diámetros 0.1 $<D<1 \mathrm{~km}$ es significativamente menor para la simulación que usa el gap más grande entre $Q_{\mathrm{S}}$ y $Q_{\mathrm{D}}$. Este resultado implica que el primer valle de la onda es más profundo para los gaps más grandes entre $Q_{\mathrm{S}}$ y $Q_{\mathrm{D}}$, llevando a una amplitud mayor para el siguiente pico y así siguiendo. Este análisis nos permite entender por qué cuanto más grande es el gap, más grande es la amplitud de la onda en la distribución de tamaños.

Por otra parte, los resultados generales indican que los tiempos de vida colisionales medios de los Troyanos jovianos de $L_{4}$ con diámetros $D \gtrsim 0.1-1 \mathrm{~km}$ resultan ser superiores usando un gap pequeño entre las leyes $Q_{\mathrm{S}}$ y $Q_{\mathrm{D}}$. La Figura 6.8 nos permite apreciar este comportamiento, mostrando resultados representativos concernientes a los tiempos de vida colisionales medios de los Troyanos con $D \gtrsim 0.1 \mathrm{~km}$, usando las leyes $Q_{\mathrm{S}, 1}$ y $Q_{\mathrm{S}, 4}$ de la Figura $6.4 \mathrm{~b}$, las cuales muestran un gap chico y grande con la ley $Q_{\mathrm{D}}$ de Benz \& Asphaug (1999), respectivamente. En efecto, de acuerdo a aquello discutido en el Capítulo 4, el diámetro $D_{\mathrm{p}}$ del proyectil más pequeño capaz de fragmentar catastróficamente un blanco con diámetro $D$ puede ser aproximado por la siguiente expresión

$$
D_{\mathrm{p}}=\left(\frac{4 Q_{\mathrm{S}}}{V^{2}}\right)^{1 / 3} D,
$$

donde $V$ es la velocidad relativa de impacto y $Q_{\mathrm{S}}$ es la energía específica de fragmentación del blanco. Para un gap grande entre $Q_{\mathrm{S}}$ y $Q_{\mathrm{D}}$, los valores de $Q_{\mathrm{S}}$ para objetos con $D \gtrsim$ 0.1-1 km son menores que aquellos asociados con un gap pequeño. De este modo, cuanto más grande es el gap, más pequeños son los valores de $D_{\mathrm{p}}$ para blancos con $D \gtrsim 0.1-1 \mathrm{~km}$. Luego, en términos generales, las simulaciones numéricas con un gap grande tienen más proyectiles capaces de fragmentar un dado blanco con $D \gtrsim 0.1-1 \mathrm{~km}$ que aquellas simulaciones con un gap pequeño. Por otra parte, la Figure 6.8 muestra que los Troyanos de mayor tamaño tienen tiempos de vida colisionales medios superiores a la edad del Sistema Solar, lo cual implica que tales asteroides probablemente han sobrevivido inalterados por impactos catastróficos 

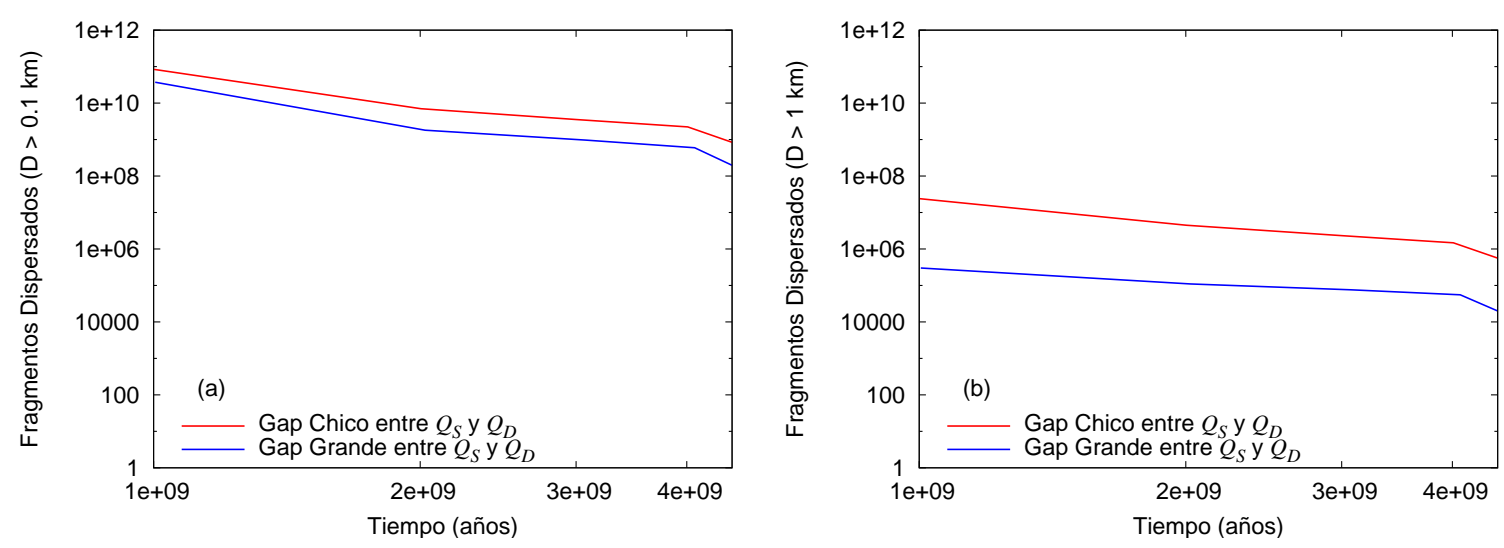

Figura 6.7. Número de cuerpos dispersados con diámetros $\geq 0.1 \mathrm{~km}$ a) y $1 \mathrm{~km} \mathrm{~b}$ ), resultantes de impactos catastróficos y eventos de craterización, como función del tiempo. Estos resultados han sido obtenidos a partir de las leyes $Q_{\mathrm{S}, 1}$ y $Q_{\mathrm{S}, 4}$ presentadas en las Figura 6.4b, las cuales muestran un gap chico y grande con la ley $Q_{\mathrm{D}}$ de Benz \& Asphaug (1999), respectivamente. Con esta ley $Q_{\mathrm{S}, 4}$, el número de fragmentos dispersados con diámetros $D>$ $0.1 \mathrm{~km}$ es $\sim 55-75 \%$ menor que aquel derivado usando la ley $Q_{\mathrm{S}, 1}$, la cual muestra el gap más pequeño. Además, para ambas simulaciones, el número de fragmentos dispersados con $D>1 \mathrm{~km}$ resulta ser algunos órdenes de magnitud menor que el número total de fragmentos dispersados con $D>0.1 \mathrm{~km}$. Con esto, más del $99 \%$ del total de los fragmentos dispersados con diámetros $D>0.1 \mathrm{~km}$ se ubica en el rango de diámetros $0.1<D<1 \mathrm{~km}$.

sobre la historia del Sistema Solar, lo cual es consistente con aquello discutido por Davis \& Weidenschilling (1981) y Marzari et al. (1997).

\subsubsection{Familias de Troyanos}

La existencia de familias de asteroides en el enjambre joviano $L_{4}$ representa una clara consecuencia de la actividad colisional en esta población. Durante los primeros años de esta década, Beaugé \& Roig (2001) desarrollaron un modelo semianalítico para el movimiento de los asteroides Troyanos. A partir de este algoritmo, elementos propios precisos fueron estimados para un conjunto de 533 Troyanos, lo cual permitió identificar la existencia de dos familias de asteroides robustas alrededor del punto $L_{4}$, conocidas como Menelaus y Epeios. Menelaus es la candidata más confiable a ser una familia real. La distribución de tamaños de sus miembros muestra sólo dos asteroides con diámetros $D \sim 80 \mathrm{~km}$, tres objetos en el rango de 40-50 km, más un gran número de pequeños cuerpos con tamaños de $\sim 20$ $30 \mathrm{~km}$. Por otra parte, la distribución de tamaños de la familia Epeios es muy diferente a aquella discutida para Menelaus, ya que todos sus miembros presentan diámetros $D \lesssim 40$ km. Algunos años después, Dotto et al. (2006) hicieron uso de la lista de familias de Troyanos jovianos suministrada por Beaugé \& Roig (2001) y estudiaron las propiedades superficiales de diversos miembros pertenecientes a las familias Menelaus, 1986 WD y Makhaon asociadas a $L_{4}$. Luego, en el marco de trabajo del mismo proyecto, Fornasier et al. (2007) analizaron las principales características de los pequeños y grandes miembros asociados a las familias de $L_{4}$ Eurybates, Menelaus, 1986 WD y 1986 TS6. Estos estudios indican que todos los miembros de la familia Eurybates, excepto el más grande cuyo diámetro $D$ es de $\sim 70 \mathrm{~km}$, son menores $\mathrm{a} \sim 40 \mathrm{~km}$. Por otra parte, la distribución de tamaños de los miembros de 1986 WD muestra 


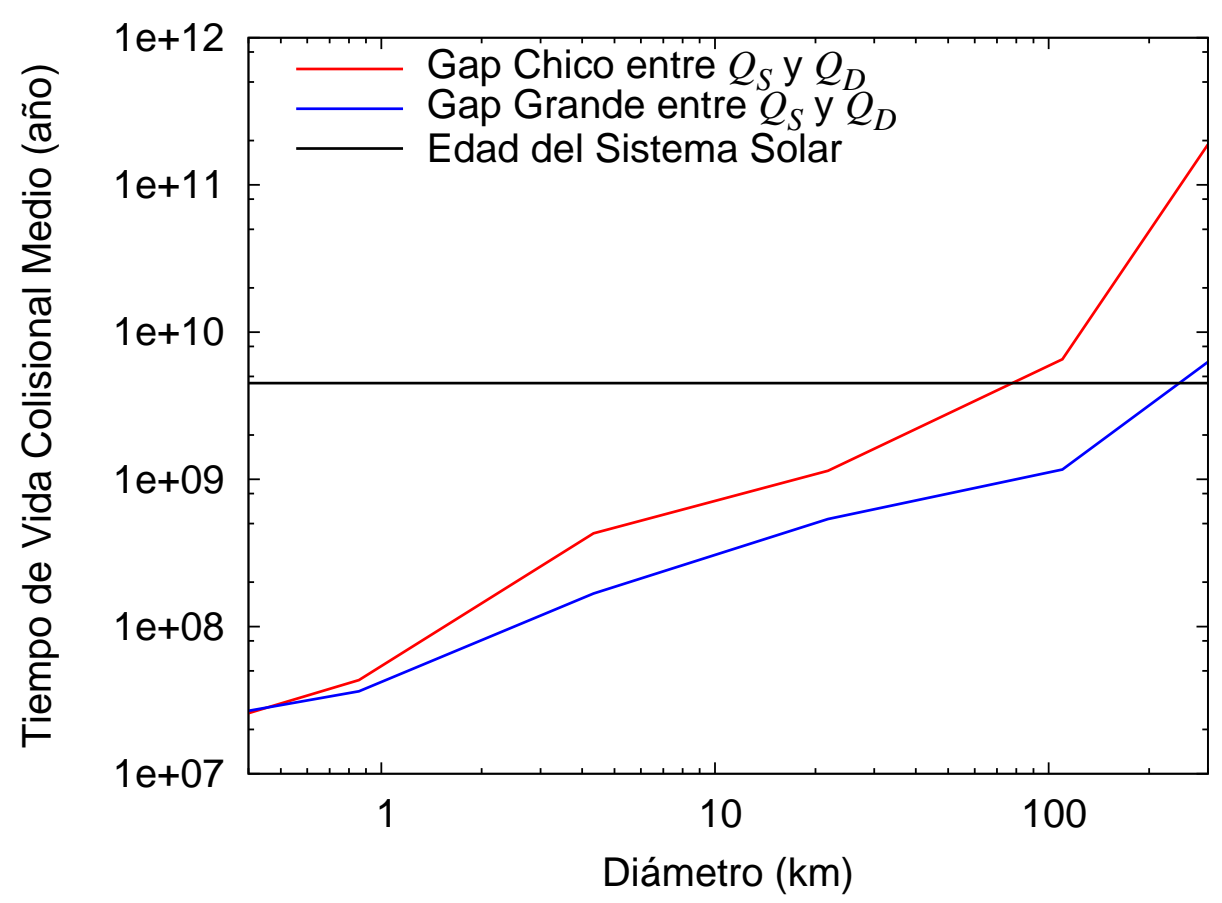

Figura 6.8. Tiempos de vida colisionales medios de los Troyanos jovianos de $L_{4}$, obtenidos usando las leyes $Q_{\mathrm{S}, 1}$ (curva roja) y $Q_{\mathrm{S}, 4}$ (curva azul) presentadas en la Figura $6.4 \mathrm{~b}$, las cuales muestran un gap chico y grande con la ley $Q_{\mathrm{D}}$ de Benz \& Asphaug (1999), respectivamente. La línea negra horizontal representa la edad del Sistema Solar. Los Troyanos de mayor tamaño representan una población primordial, ya que sus tiempos de vida colisionales medios son superiores a los $4.5 \times 10^{9}$ años. Esto es consistente con aquello discutido por Davis \& Weidenschilling (1981) y Marzari et al. (1997).

unos pocos objetos con diámetros $D \gtrsim 50 \mathrm{~km}$, más algunos cuerpos con tamaños menores a los $40 \mathrm{~km}$. Además, todos los miembros de las familias Makhaon y 1986 TS6 presentan diámetros $D \lesssim 55 \mathrm{~km}$.

Dadas las distribuciones de tamaños de los miembros de las familias de $L_{4}$ estudiadas por Beaugé \& Roig (2001), Dotto et al. (2006) y Fornasier et al. (2007), decidimos analizar la formación de familias de Troyanos a partir de la fractura de cuerpos precursores con diámetros $D>50$ y $100 \mathrm{~km}$, que dispersen fragmentos de $\sim 40 \mathrm{~km}$. Haciendo uso de la población inicial PI1, las simulaciones con los más grandes gaps entre $Q_{\mathrm{S}}$ y $Q_{\mathrm{D}}$ no forman ninguna de tales familias, lo cual elimina aquellas leyes $Q_{\mathrm{S}}$ como posibles energías específicas de fragmentación para los Troyanos jovianos de $L_{4}$. Por otra parte, las simulaciones con los más pequeños gaps entre $Q_{\mathrm{S}}$ y $Q_{\mathrm{D}}$, excepto aquella que usa la ley $Q_{\mathrm{S}, 1}$ de la Figura $6.4 \mathrm{~b}$, llevan a la formación de un máximo de 2 familias de Troyanos a partir de cuerpos precursores con diámetros $D>$ $100 \mathrm{~km}$, aunque no producen familias a partir de objetos en el rango de 50 a $100 \mathrm{~km}$. Usando la ley $Q_{\mathrm{S}, 1}$ de la Figura 6.4b, 7 familias de Troyanos son formadas a partir de la fractura de cuerpos con diámetros $D>100 \mathrm{~km}$, mientras que 4 objetos con diámetros $D$ en el rango 50 $<D<100 \mathrm{~km}$ son fragmentados catastróficamente sobre $4.5 \times 10^{9}$ años. Estos resultados son consistentes con el número de familias de $L_{4}$ estudiadas por Beaugé \& Roig (2001), Dotto et al. (2006) y Fornasier et al. (2007), sugiriendo además que nuevas familias deberían ser 
identificadas en el futuro.

Vale la pena notar que el proceso de formación de familias analizado aquí resulta ser muy poco sensible a la distribución de tamaños inicial, ya que no se obtienen cambios significativos en los resultados haciendo uso de la población inicial PI2.

\subsubsection{Tasas de Eyección desde el Punto de Equilibrio Joviano $L_{4}$}

Las Figuras 6.9a, b, c y d muestran la evolución en el tiempo del número de cuerpos eyectados desde el enjambre joviano $L_{4}$ con diámetros $D>1 \mathrm{~km}$ por millón de años, obtenida a partir de las leyes $Q_{\mathrm{S}}$ presentadas en las Figuras 6.4a, b, c y d, respectivamente. Los resultados ilustrados en dicha figura son aquellos derivados haciendo uso de la población inicial PI1. En términos generales, los más grandes gaps entre $Q_{\mathrm{S}}$ y $Q_{\mathrm{D}}$ llevan a las más pequeñas tasas de eyección de fragmentos Troyanos desde la nube $L_{4}$. Para entender este comportamiento, analizamos los resultados concernientes a la dispersión de fragmentos obtenidos a partir de las dos simulaciones numéricas desarrolladas en la Sección 6.6.1, usando las leyes $Q_{\mathrm{S}, 1}$ y $Q_{\mathrm{S}, 4}$ de la Figura $6.4 \mathrm{~b}$, las cuales muestran un gap chico y grande con la ley $Q_{\mathrm{D}}$ de Benz \& Asphaug (1999), respectivamente. Nuestros estudios nos permiten inferir que, para el gap más grande entre $Q_{\mathrm{S}}$ y $Q_{\mathrm{D}}$, el número de fragmentos dispersados con diámetros $D>1 \mathrm{~km}$, resultantes de eventos catastróficos y de craterización, es $\sim 95 \%$ menor que aquel obtenido a partir del experimento numérico que usa el gap más chico (Figura 6.7b). Esto indica que la simulación con el gap más grande entre $Q_{\mathrm{S}}$ y $Q_{\mathrm{D}}$ produce un número significativamente menor de fragmentos con $D>1 \mathrm{~km}$ que pueden ser eyectados del enjambre $L_{4}$, comparado con aquel derivado con el gap más chico. Esto nos permite entender la tendencia general de nuestros resultados concernientes a la tasa de eyección de fragmentos Troyanos desde $L_{4}$. Por otra parte, destacamos que la tasa de eyección de fragmentos colisionales con diámetros $D>$ $1 \mathrm{~km}$ es poco sensible a la distribución de tamaños inicial, ya que no se observan cambios significativos en los resultados derivados haciendo uso de la población inicial PI2.

En la Sección 6.5, discutimos diversos criterios para determinar el destino final de los fragmentos Troyanos y a partir de esto calcular las tasas de eyección desde el enjambre joviano $L_{4}$. A partir de todos nuestros experimentos numéricos, encontramos que los fragmentos que escapan de $L_{4}$ presentan excentricidades $e<1$ (Figura 6.10a), lo cual elimina la eyección colisional hiperbólica como una posible fuente de remoción de Troyanos. Por otra parte, cuando un impacto ocurre, el valor absoluto de la diferencia entre las longitudes medias inicial y final de los Troyanos colisionantes es siempre menor que la amplitud de libración media de $\sim 30^{\circ}$, lo cual indica que las colisiones en el enjambre $L_{4}$ no permiten la eyección de fragmentos a partir de cambios relevantes en el comportamiento libracional. Para todas nuestras simulaciones, la eyección de fragmentos Troyanos desde $L_{4}$ es debida a variaciones en los valores de $a$, e e $i$, los cuales se asocian a algunos de los nichos de inestabilidad mostrados en la Figura 6.3 o exceden los límites del enjambre (Sección 6.3). Para ilustrar la distribución de los fragmentos eyectados desde $L_{4}$ en el espacio de los elementos orbitales, dividimos los planos $(a, e)$ y $(a, i)$ en nichos, similares a aquellos definidos en la Sección 6.3. Luego, el número de fragmentos en cada nicho es normalizado respecto del total que son eyectados. Este procedimiento nos permite construir el mapa de la Figura 6.10, el cual es representativo de la distribución de Troyanos eyectados desde $L_{4}$, con respecto al semieje mayor, excentricidad e inclinación. 

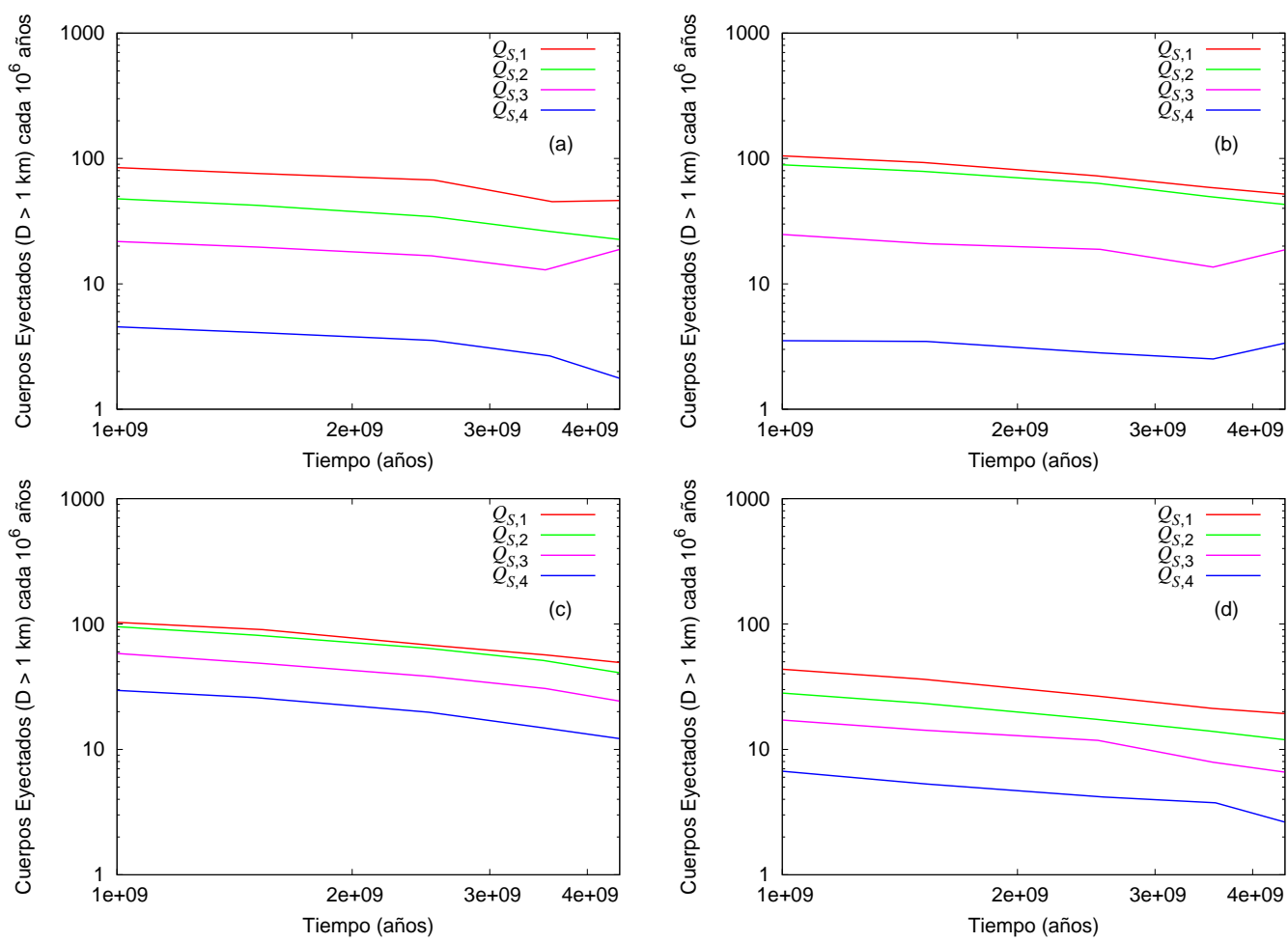

Figura 6.9. Las tasas de eyección de Troyanos jovianos de $L_{4}$ con diámetros $D>1 \mathrm{~km}$ por millón de años, obtenidas a partir de las leyes $Q_{\mathrm{S}}$ presentadas en las Figuras 6.4a, b, c y d son mostradas en a), b), c) y d), respectivamente. Notemos que este conjunto de simulaciones numéricas ha sido desarrollado haciendo uso de la población inicial PI1. En términos generales, los más grandes gaps entre $Q_{\mathrm{S}}$ y $Q_{\mathrm{D}}$ llevan a las más pequeñas tasas de eyección colisional de fragmentos desde la nube $L_{4}$. Por otra parte, la tasa máxima de eyección actual desde $L_{4}$ es de $\sim 50$ objetos con diámetros $D>1 \mathrm{~km}$ por millón de años. Destacamos que la tasa de eyección de fragmentos colisionales con diámetros $D>1$ $\mathrm{km}$ es poco sensible a la distribución de tamaños inicial, ya que no se observan cambios significativos en los resultados derivados usando la población inicial PI2.

Por otra parte, para todos los casos, la mayoría de los cuerpos eyectados con diámetros mayores a $1 \mathrm{~km}$ desde el enjambre joviano $L_{4}$ tienen diámetros entre 1 y $5 \mathrm{~km}$. En efecto, nuestras simulaciones muestran que el número de fragmentos con diámetros mayores a $5 \mathrm{~km}$ removidos por unidad de tiempo desde $L_{4}$ resulta ser despreciable.

Uno de los objetivos más importantes de este trabajo es analizar una posible conexión entre los fragmentos Troyanos que escapan de $L_{4}$ y las poblaciones de Centauros y Cometas de la Familia de Júpiter. Tal como ya hemos mencionado, la existencia de alguna conexión genética entre los asteroides Troyanos jovianos y los Cometas de Corto Período fue sugerida por Hartmann et al. (1987), Shoemaker et al. (1989), Jewitt \& Luu (1990) y Fitzsimmons et al. (1994), quienes desarrollaron estudios espectroscópicos y encontraron similitudes entre los cometas y los asteroides tipo $D$, los cuales representan la clase más abundante entre Troyanos. Además, la distribución de albedos de los Troyanos jovianos muestra similitudes con aquella correspondiente a los cometas (Fernández et al. 2003), pero resulta ser inconsistente con las distribuciones asociadas a Centauros y objetos de la Región Transneptuniana (Stansberry et al. 2008). 

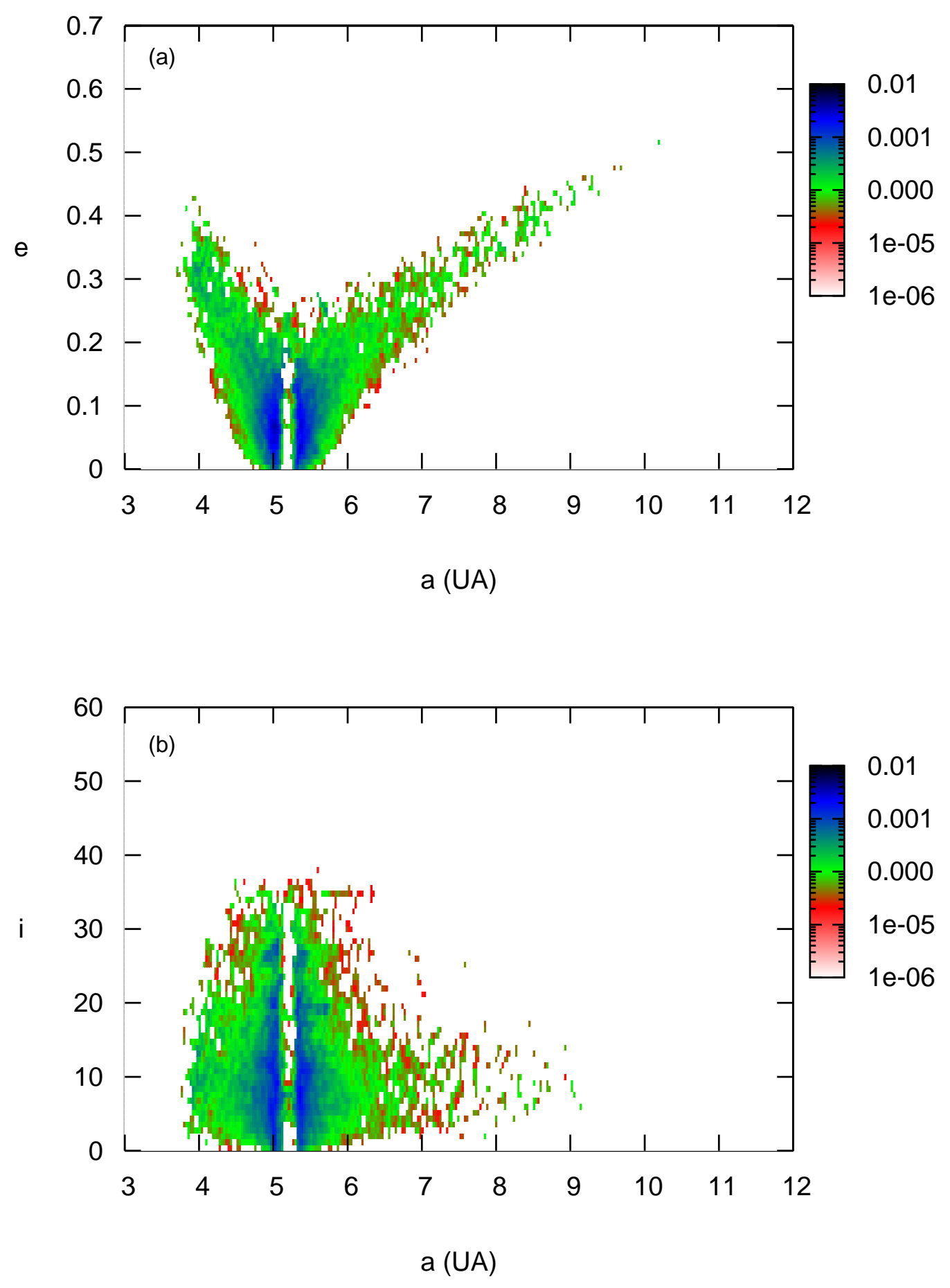

Figura 6.10. Distribución normalizada de los Troyanos eyectados desde el enjambre joviano $L_{4}$ en función del semieje mayor, excentricidad e inclinación. 
Recientemente, Di Sisto \& Brunini (2007) analizaron el origen y la distribución de la población de Centauros. Estos autores infirieron que los objetos del Disco Dispersado de la Región Transneptuniana son probablemente la fuente principal de Centauros, suministrando una tasa actual de $\sim 4 \times 10^{6}$ objetos con un radio $R>1 \mathrm{~km}$ por millón de años. Además, sus resultados indican que el $30 \%$ de los objetos del Disco Dispersado que entran a la zona de Centauros alcanzan la región interior a la órbita de Júpiter, obteniendo una tasa actual de $\sim$ $1 \times 10^{6}$ Cometas de la Familia de Júpiter con un radio $R>1 \mathrm{~km}$ por millón de años. Con el objetivo de estudiar la contribución de los Troyanos a las poblaciones actuales de Centauros y Cometas de la Familia de Júpiter, estimamos una tasa media de eyección de fragmentos desde $L_{4}$ para cada una de nuestras simulaciones sobre los últimos $5 \times 10^{8}$ años de evolución, donde el número de cuerpos removidos por unidad de tiempo es más o menos constante y la muestra de datos resulta ser estadísticamente significativa. Uno de nuestros principales resultados es que la tasa máxima de eyección desde $L_{4}$, correspondiente al gap más pequeño entre $Q_{\mathrm{S}}$ y $Q_{\mathrm{D}}$, es de $\sim 50$ objetos con diámetros $D>1 \mathrm{~km}$ por millón de años. Este valor es mucho menor que aquel obtenido previamente por Marzari et al. (1997), quienes derivaron una tasa de eyección colisional de $\sim 3600$ objetos con diámetros $D$ en el rango $1<D<40 \mathrm{~km}$ por millón de años desde $L_{4}$. De acuerdo a estos resultados, esperaríamos un número máximo de 1 Centauro o Cometa de la Familia de Júpiter con un diámetro $D>1 \mathrm{~km}$ cada 20000 años desde el enjambre joviano $L_{4}$, mientras que las estimaciones de Di Sisto \& Brunini (2007) sugieren que cada año, 4 Centauros y 1 Cometa de la Familia de Júpiter con un radio $R>$ $1 \mathrm{~km}$ son eyectados desde el Disco Dispersado de la Región Transneptuniana. A partir de esto, concluimos que la contribución de los asteroides Troyanos a las poblaciones actuales de Centauros y Cometas de la Familia de Júpiter resulta ser despreciable.

\subsubsection{Polvo Troyano}

La existencia de polvo en nuestro Sistema Solar ha sido conocida desde tiempo remotos a partir de observaciones de la luz zodiacal. En el Sistema Solar interior, el polvo es producido por restos de asteroides y Cometas de la Familia de Júpiter (Dermott et al. 1994; Liou et al. 1995). La dispersión de la luz por parte de estos granos da lugar a la luz zodiacal y su emisión térmica domina el cielo de la noche entre los 5 y los $500 \mu \mathrm{m}$. Esta emisión térmica fue observada por los Telescopios Espaciales IRAS y COBE, y las partículas de polvo interplanetarias fueron detectadas a partir de instrumentos sobre las naves espaciales Pioneer $10 \mathrm{y}$ 11, Voyager, Galileo y Ulyses. Dermott et al. (2002) estimaron que la luminosidad del polvo del Sistema Solar interior es $\sim 10^{-8}-10^{-7} L_{\odot}$, siendo $L_{\odot}$ la luminosidad solar. En el Sistema Solar exterior, la emisión térmica del polvo no fue detectada por los Telescopios Espaciales IRAS y COBE debido a la presencia de una componente mucho más fuerte asociada a la luz zodiacal. Sin embargo, las naves espaciales Pioneer 10 y 11 dieron evidencia de la existencia de partículas de polvo más allá de la órbita de Saturno (Landgraf et al. 2002). Se especula que en el Sistema Solar exterior debe existir una tasa de producción de polvo significativa debido a las mutuas colisiones entre objetos de la Región Transneptuniana y colisiones con granos interestelares (Backman \& Paresce 1993; Stern 1996; Yamamoto \& Mukai 1998). Extrapolando la distribución de tamaños de los objetos de la Región Transneptuniana, Stern (1996) estimó que la luminosidad del polvo del Sistema Solar exterior es $\sim 10^{-7}-10^{-6} L_{\odot}$.

Las colisiones entre asteroides producen un gran número de partículas de polvo con diámetros $D \lesssim 500 \mu \mathrm{m}$, en todo el Sistema Solar. Los tiempos de vida de estos pequeños granos 
de polvo están afectados fundamentalmente por diversos mecanismos de radiación, tales como el efecto Poynting-Robertson, la presión de radiación y el viento solar (Burns et al. 1979).

Aquí, nuestro objetivo es calcular la luminosidad del polvo asociado a la población de Troyanos jovianos de $L_{4}$ y determinar el grado de su contribución frente a los valores ya expuestos para la emisión térmica del polvo del Sistema Solar interior y exterior ${ }^{2}$.

Para llevar a cabo este estudio, necesitamos realizar una modificación del código numérico que utilizamos en este capítulo. De acuerdo a lo hecho hasta aquí y en consistencia con aquello sugerido por Campo Bagatin et al. (1994a) y Campo Bagatin (1998), nuestro algoritmo no evoluciona en el tiempo los primeros 60 bines de tamaño, cuyos diámetros centrales van desde $10^{-10}$ a $10^{-4} \mathrm{~km}$. Sin embargo, en esta ocasión, estamos interesados en analizar el comportamiento de las partículas con diámetros entre 5 y $500 \mu \mathrm{m}$, por lo que la versión modificada de nuestro código no evoluciona en el tiempo los primeros 18 bines de tamaño, cuyos diámetros centrales van desde $10^{-10}$ a $5 \times 10^{-9} \mathrm{~km}$ (esto es, desde 0.1 a $5 \mu \mathrm{m}$ ). Luego, de modo equivalente a lo que hemos hecho, esta parte de la población es usada únicamente a modo de proyectiles, y su distribución de tamaños es determinada en cada paso de tiempo, extrapolando la pendiente de la distribución asociada a los 10 bines de tamaño siguientes.

Una vez hecho esto, el procedimiento para analizar la evolución colisional de la población de Troyanos de $L_{4}$ y determinar el destino final de los fragmentos generados es aquel descripto en la Sección 6.5. Sin embargo, una vez que aplicamos este algoritmo y derivamos la variación en el número de cuerpos $\Delta N_{k}$, la remoción de pequeñas partículas debido a la acción de fuerzas de radiación debe ser incluida en el modelo, en cada paso de tiempo. Burns et al. (1979) discutieron los principales efectos de las fuerzas de radiación solar tales como la presión de radiación, el efecto Poynting-Robertson, el viento solar, el efecto Yarkovsky y el efecto Doppler diferencial. Estos autores mostraron que sólo partículas con tamaños de $\sim 0.1 \mu \mathrm{m}$ compuestas de hierro, magnetita y grafito pueden ser fácilmente eyectadas del Sistema Solar debido a la presión de radiación. Partículas con tamaños menores y mayores que $\sim 0.1 \mu \mathrm{m}$ resultan ser más estables frente a la eyección por presión de radiación. Sin embargo, Burns et al. (1979) indicaron que partículas de mayor tamaño son arrastradas hacia el Sol por el efecto Poynting-Robertson, mientras que aquellas de menor tamaño son removidas por la acción del viento solar. Por otra parte, estos autores mostraron que la magnitud del efecto Doppler diferencial es siempre menor que la magnitud del efecto Poynting-Robertson, mientras que la acción del efecto Yarkovsky, dependiendo de las propiedades del material, domina otras perturbaciones disipativas para cuerpos con tamaños de $\sim 1 \mathrm{~m}$. De acuerdo con esto, el efecto Poynting-Robertson es el mecanismo más importante que afecta a las partículas de polvo. Klačka (1992) desarrolló un trabajo interesante en relación a este efecto.

Según el estudio realizado por Burns et al. (1979), la escala de tiempo de remoción de un grano de polvo debido al efecto Poyinting-Robertson está dada por

$$
t_{\mathrm{PR}}=710\left(\frac{R}{\mu \mathrm{m}}\right)\left(\frac{\rho}{\mathrm{gr} / \mathrm{cm}^{3}}\right)\left(\frac{r}{\mathrm{UA}}\right)^{2}\left(\frac{1}{1+A}\right) \text { años, }
$$

donde $R, \rho, A$ y $r$ representan el radio, la densidad, el albedo y la distancia al Sol del grano de polvo, respectivamente. A partir de esto, la variación total en el número de cuerpos $N_{k}$ asociados a un dado bin de tamaño $k$ de la población de Troyanos, en un intervalo $\Delta t$, y teniendo

\footnotetext{
${ }^{2}$ En este estudio, sólo incluimos los efectos de la emisión térmica del polvo Troyano joviano. El scattering de la luz del Sol producido por estas partículas no es tenido en cuenta.
} 


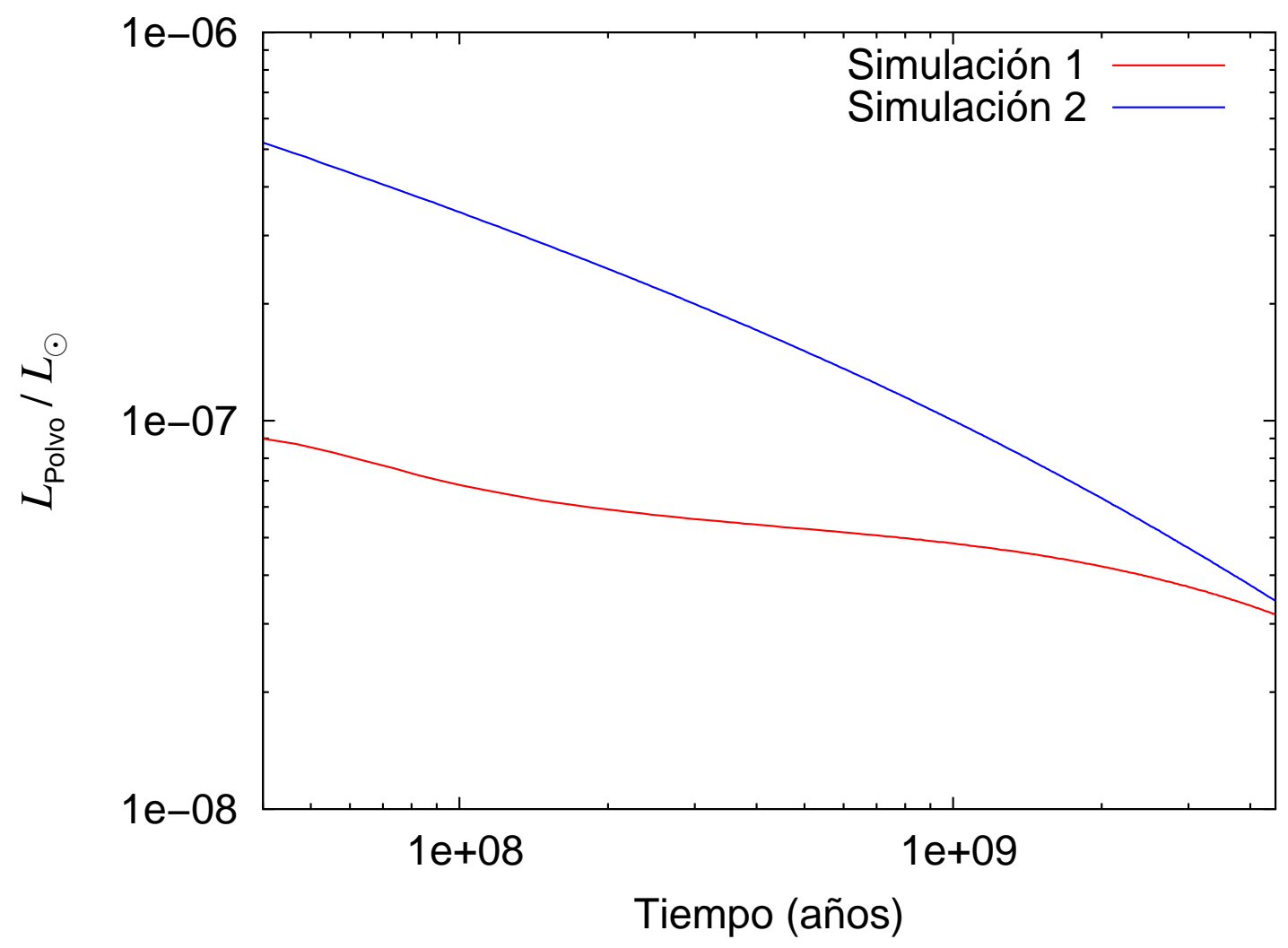

Figura 6.11. Luminosidad del polvo Troyano joviano $L_{\text {Polvo }}$ en el enjambre $L_{4}$, relativa a la luminosidad solar $L_{\odot}$, como función del tiempo. El valor actual de $L_{\text {Polvo }}$ es muy poco sensible a variaciones en la distribución de tamaños inicial, encontrándose en el rango $3.2-3.4 \times 10^{-8} L_{\odot}$.

en cuenta procesos de remoción colisionales, dinámicos y el efecto Poynting-Robertson, está dada por

$$
\left(\Delta N_{k}\right)_{\text {Total }}=\left(\Delta N_{k}-\frac{N_{k}}{t_{\mathrm{PR}}}\right) \Delta t
$$

Recién en este punto, el paso de tiempo $\Delta t$ es calculado en una forma tal que el cambio relativo en el número de objetos en cualquier bin de tamaño sea siempre menor que el $1 \%$.

El área de la nube de polvo existente en el enjambre joviano $L_{4}$ puede ser expresada por

$$
A_{\text {Polvo }}=\frac{\pi}{4} \sum_{D=5 \mu \mathrm{m}}^{500 \mu \mathrm{m}} D^{2} N(D),
$$

donde $N(D)$ es el número de partículas con diámetro $D$. La luminosidad del polvo $L_{\text {Polvo }}$ relativa a la luminosidad solar $L_{\odot}$ está dada por la siguiente expresión

$$
\frac{L_{\text {Polvo }}}{L_{\odot}}=\left(\frac{A_{\text {Polvo }}}{4 \pi R_{\odot}^{2}}\right)\left(\frac{T_{\text {Polvo }}}{T_{\odot}}\right)^{4},
$$


donde $R_{\odot}$ es el radio del Sol, y $T_{\text {Polvo }}$ y $T_{\odot}$ son las temperaturas efectivas de las partículas de polvo en el punto $L_{4}$ y el Sol, respectivamente. Para obtener $T_{\text {Polvo }}$, procedemos de modo equivalente a lo hecho en la Sección 5.4.2. En efecto, igualando el flujo solar absorvido al flujo emitido por la partícula de polvo, obtenemos que

$$
\begin{aligned}
\pi R^{2}(1-A) S & =4 \pi R^{2} \epsilon \sigma T_{\text {Polvo }}^{4} \\
T_{\text {Polvo }} & =\left[\frac{(1-A) S}{4 \epsilon \sigma}\right]^{1 / 4},
\end{aligned}
$$

donde $\sigma=5.67 \times 10^{-8} \mathrm{~W} \mathrm{~m}^{-2} \mathrm{~K}^{-4}$ es la constante de Stefan-Boltzmann, $A$ es el albedo (en este caso, asumido igual a 0.04), $\epsilon$ es la emisividad (asumida igual a 1), y $S$ es el flujo solar en la posición de la partícula. Como ya indicamos en la Sección 5.4.2, $S$ depende del semieje mayor y puede ser expresado por la fórmula

$$
S=S_{0}\left(\frac{a_{0}}{a}\right)^{2}
$$

donde $S_{0}=1370 \mathrm{~W} \mathrm{~m}^{-2}$ es la constante solar, y $a_{0}$ y $a$ son los semiejes mayores de la Tierra y la partícula bajo consideración, respectivamente. Para los Troyanos jovianos, $a=5.2$ UA y, de acuerdo a la Ecuación 6.19, la temperatura efectiva $T_{\text {Polvo }}$ de las partículas de polvo en el enjambre $L_{4}$ es igual a $122.25 \mathrm{~K}$. A partir de esto, y teniendo en cuenta que $R_{\odot}=7 \times 10^{5}$ $\mathrm{km} \mathrm{y} T_{\odot}=5800 \mathrm{~K}$, la Ecuación 6.17 adopta la forma

$$
\frac{L_{\text {Polvo }}}{L_{\odot}}=3,2 \times 10^{-20}\left(\frac{A_{\text {Polvo }}}{\mathrm{km}^{2}}\right) .
$$

Como se aprecia, la luminosidad del polvo es una función del área de la nube de polvo $A_{\text {Polvo }}$. De acuerdo a la Ecuación $6.16, A_{\text {Polvo }}$ depende del número de partículas de la población de Troyanos de $L_{4}$ entre 5 y $500 \mu \mathrm{m}$. A partir de esto, resulta evidente que $L_{\text {Polvo }}$ es dependiente de la población inicial y los parámetros colisionales de nuestro modelo.

Para testear la dependencia de nuestras simulaciones con la distribución de tamaños inicial, desarrollamos corridas haciendo uso de las poblaciones iniciales PI1 y PI2 definidas en la Sección 6.2. Por otra parte, si bien realizamos corridas con diferentes leyes $Q_{\mathrm{S}}$, los resultados mostrados aquí serán aquellos obtenidos con la ley $Q_{\mathrm{S}, 1}$ de la Figura $6.4 \mathrm{~b}$, la cual muestra un pequeño gap respecto de la ley $Q_{\mathrm{D}}$ de Benz \& Asphaug (1999). Como hemos visto ya, esta ley maximiza la producción de familias y la tasa de escape de fragmentos colisionales desde el enjambre Troyano joviano $L_{4}$. De acuerdo a lo dicho, vamos a analizar de manera detallada los resultados de dos simulaciones numéricas diferentes, designadas como 1 y 2 , las cuales usan las poblaciones iniciales PI1 y PI2, respectivamente, y la ley $Q_{\mathrm{S}, 1}$ de la Figura 6.4b. Finalmente, discutiremos brevemente la dependencia de nuestras simulaciones, concernientes a la luminosidad del polvo Troyano joviano, en relación a la ley $Q_{\mathrm{S}}$.

La Figura 6.11 expone la luminosidad del polvo $L_{\text {Polvo }}$ en el enjambre joviano $L_{4}$ relativa a la luminosidad solar, como función del tiempo, obtenida a partir de cada una de las dos simulaciones numéricas que desarrollamos. En la misma es posible observar que la luminosidad actual del polvo en el enjambre $L_{4}$ es equivalente en las dos diferentes corridas. De este modo, concluimos que la evolución en el tiempo de $L_{\text {Polvo }}$ tiene las características de un proceso de difusión ya que su valor actual resulta ser muy poco sensible a variaciones en la distribución de tamaños inicial. Por otra parte, nuestros resultados indican que la luminosidad actual del 
polvo $L_{\text {Polvo }}$ en el enjambre joviano $L_{4}$ adopta valores entre $\sim 3.2 \times 10^{-8}$ y $3.4 \times 10^{-8} L_{\odot}$. De acuerdo a estos valores, concluimos que la luminosidad del polvo en el enjambre joviano $L_{4}$ resulta ser comparable a la luminosidad del polvo en el Sistema Solar interior $\left(\sim 10^{-8}-10^{-7}\right.$ $\left.L_{\odot}\right)$ y, al menos, un orden de magnitud más baja que la luminosidad del polvo asociado al Sistema Solar exterior $\left(\sim 10^{-7}-10^{-6} L_{\odot}\right)$.

Posteriormente, realizamos diversas corridas usando diferentes leyes $Q_{\mathrm{S}}$ tomadas de la Figura 6.4. Nuestros resultados muestran que el valor actual de la luminosidad del polvo en el enjambre joviano $L_{4}$ no manifiesta cambios significativos ante variaciones de $Q_{\mathrm{S}}$.

Finalmente, nosotros testeamos el rol del efecto Poynting-Robertson. Nustras simulaciones sugieren que los tiempos de vida colisionales medios de las partículas de polvo son más cortos que la escala de tiempo de remoción debido al efecto Poynting-Robertson. De este modo, la evolución del polvo Troyano joviano de $L_{4}$ está dominado por colisiones, de modo que nuestros resultados no cambian significativamente cuando el efecto Poynting-Robertson es removido de nuestras simulaciones.

\subsubsection{Robustez de los Resultados}

Los resultados mostrados en la Sección 6.6 han sido obtenidos usando los nichos de estabilidad e inestabilidad definidos en la Sección 6.3, los cuales presentan anchos de 0.02 UA, 0.0125 y $2.25^{\circ}$ en semieje mayor $a$, excentricidad $e$ e inclinación $i$, respectivamente. Con el objetivo de testear la dependencia de nuestros resultados sobre el tamaño de aquellos nichos, llevamos a cabo diversos experimentos numéricos incrementando los anchos de tales regiones en $a, e$ e $i$, lo cual lleva a magnificar la región de estabilidad. En términos generales, cuanto más grande es el área de los nichos, más pequeña es la tasa de eyección de fragmentos desde el enjambre joviano $L_{4}$. En este estudio, seleccionamos nichos pequeños con el objetivo de minimizar la influencia de los Troyanos aislados en la distribución de la población. Por otra parte, encontramos que los resultados concernientes a la distribución de tamaños de los Troyanos de $L_{4}$ y la formación de familias no son sensibles al tamaño de las regiones de estabilidad e inestabilidad construidas para desarrollar nuestro tratamiento dinámico.

Finalmente, vale la pena recordar que los resultados presentados en este estudio han sido derivados generando valores iniciales del semieje mayor $a$, excentricidad $e$ e inclinación $i$ a partir de la distribución de Troyanos de $L_{4}$ mostrada en la Figura 6.3. Con el objetivo de testear la dependencia de nuestros resultados sobre la distribución orbital inicial, desarrollamos diversas simulaciones comenzando con una población dinámicamente fría, con excentricidades e inclinaciones menores a 0.05 y $10^{\circ}$, respectivamente. Tales valores límites de $e$ e $i$ son seleccionados al azar. Nuestros resultados muestran que fragmentos Troyanos con diámetros $D>1 \mathrm{~km}$ requieren escalas de tiempo del orden de $10^{8}$ años para alcanzar la configuración orbital actual (Figura 6.12), mientras que fragmentos más pequeños ocupan los nichos de estabilidad muy rápidamente, en sólo algunos miles de años. Además, encontramos que los resultados concernientes a la distribución de tamaños de los Troyanos de $L_{4}$, sus tiempos de vida colisionales medios, la tasa de eyección de fragmentos desde $L_{4}$ y la formación de familias no muestran una fuerte dependencia sobre la distribución inicial de elementos orbitales. A partir de este análisis, inferimos que la distribución orbital actual de los asteroides Troyanos no ofrece una fuerte restricción sobre el origen dinámico de esta población. 

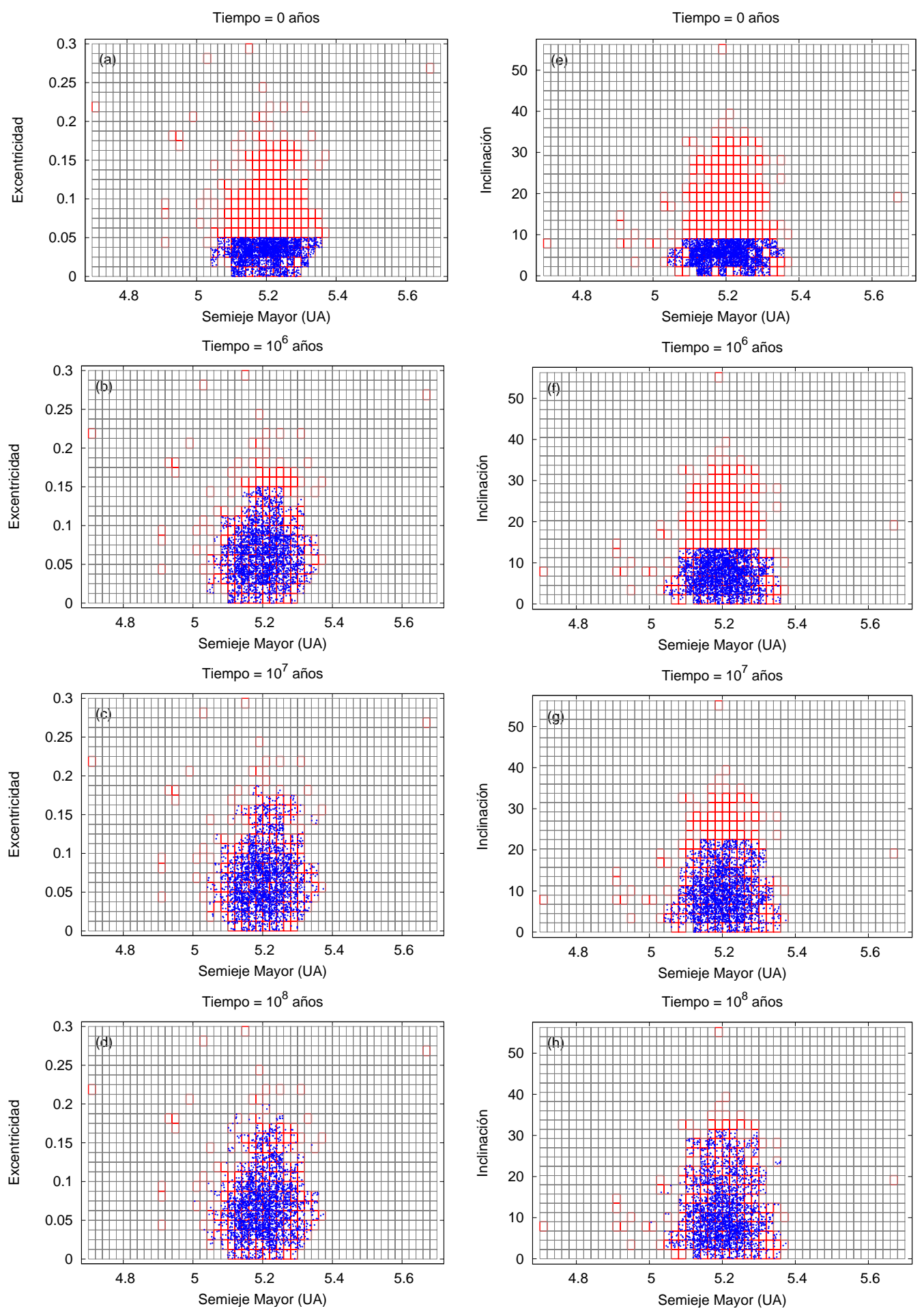

Figura 6.12. Distribución de fragmentos de Troyanos con diámetros $D>1 \mathrm{~km}$ con respecto al semieje mayor $a$, excentricidad $e$ e inclinación $i$, como función del tiempo. 


\section{Capítulo 7}

\section{Plutinos}

\subsection{Introducción}

Los resultados presentados en este capítulo fueron publicados en Astronomy \& Astrophysics, y pueden encontrarse bajo la referencia de Elía, Brunini \& Di Sisto (2008).

Tal como indicamos en la Sección 3.1.5, si bien es ampliamente aceptado que el Disco Dispersado de la Región Transneptuniana es la fuente primaria de Centauros y Cometas de la Familia de Júpiter (Duncan \& Levison 1997; Di Sisto \& Brunini 2007), diversos autores también han sugerido la existencia de alguna conexión entre los Plutinos y los cometas. En efecto, Duncan et al. (1995) analizaron la estructura dinámica de la Región Transneptuniana y encontraron que en la resonancia de movimientos medios 3:2 con Neptuno existen órbitas que resultan ser inestables sobre escalas de tiempo comparables a la edad del Sistema Solar. Ya que los tiempos de vida dinámicos de los cometas resultan ser muy cortos, aquellos que hoy observamos deben haber abandonado sus regiones fuentes sólo unos pocos millones de años atrás. La existencia de aquellas órbitas en la resonancia de movimientos medios 3:2 con Neptuno les permitió sugerir a Duncan et al. (1995) que esta región resonante podría estar relacionada con el origen de los Cometas de la Familia de Júpiter observados.

Motivado por estos resultados, Morbidelli (1997) estudió la dinámica de la resonancia 3:2 con Neptuno a bajas inclinaciones y mostró la existencia de una región débilmente difusiva a moderadas amplitudes de libración, la cual debería ser una fuente activa de cometas en el tiempo presente. Por otra parte, Morbidelli (1997) estimó que $\sim 4.5 \times 10^{8}$ objetos de tamaño cometario deberían estar atrapados actualmente en la resonancia 3:2 para reproducir el flujo observado de Cometas de la Familia de Júpiter en el Sistema Solar interior. Este número parece indicar que los Plutinos deberían representar una población colisionalmente evolucionada.

El primer intento de incluir la influencia gravitacional de Plutón dentro de los modelos numéricos de la dinámica de Plutinos fue desarrollado por Yu \& Tremaine (1999). Estos autores sugirieron que el efecto de Plutón, eyectando objetos desde la resonancia 3:2 hacia órbitas de cruce con Neptuno, podría contribuir significativamente al flujo de Cometas de la Familia de Júpiter. Al mismo tiempo, Nesvorný et al. (2000) mostraron, a través de simulaciones numéricas, que además de los cuatro planetas gigantes, Plutón debería ser incluido en los modelos destinados a analizar la estructura dinámica de la Región Transneptuniana, siendo su efecto 
particularmente importante sobre los objetos de la resonancia 3:2 con Neptuno. En efecto, estos autores encontraron que Plutón puede producir una gran excitación en las amplitudes de libración de los Plutinos, los cuales podrían ser eyectados de la resonancia y contribuir a la población cometaria.

Paralelamente, Melita \& Brunini (2000) desarrollaron un estudio comparativo de las principales resonancias de movimientos medios de la Región Transneptuniana. En efecto, estos autores describieron la estructura dinámica de las resonancias 3:2, 5:3 y 2:1 con Neptuno, a 39.5, 42.3 y 47.7 UA, respectivamente. Particularmente, Melita \& Brunini (2000) mostraron que la resonancia 3:2 presenta una zona estable muy robusta principalmente a bajas inclinaciones, donde la mayoría de los Plutinos observados se encuentran distribuidos. Además, ellos sugirieron que la existencia de Plutinos en regiones muy inestables puede ser explicada por colisiones o encuentros gravitacionales con otros Plutinos, o quizás asumiendo que ellos son objetos en un estado de transición. Al mismo tiempo, Nesvorný \& Roig (2000) exploraron la dinámica de la resonancia 3:2 con Neptuno, determinando sus más importantes resonancias internas. Además, estos autores estimaron que el número actual de Plutinos con diámetros $D \gtrsim 1-3 \mathrm{~km}$ es $\sim 6 \times 10^{8}$. Por otra parte, Nesvorný \& Roig (2000) mostraron que las regiones de estabilidad orbital de la resonancia 3:2 parecen no estar bien representadas por los Plutinos observados, lo cual podría ser una característica dinámica primordial o el resultado de colisiones y encuentros gravitacionales.

Más tarde, Dell'Oro et al. (2001) computaron los valores de la probabilidad intrínseca de colisión y la velocidad media de impacto para diversas poblaciones de pequeños cuerpos, incluyendo los Plutinos. Para llevar a cabo esta tarea, estos autores adoptaron el mismo formalismo matemático usado por Dell'Oro et al. (1998), el cual está basado en el método estadístico de Dell'Oro \& Paolicchi (1998). Para los Plutinos, Dell'Oro et al. (2001) obtuvieron estimaciones confiables de la probabilidad intrínseca de colisión y la velocidad media de impacto, ya que la muestra disponible en aquel tiempo permitió un buen conocimiento de la distribución de estos objetos en el espacio de las fases.

Algunos años más tarde, Bernstein et al. (2004), Elliot et al. (2005) y Petit et al. (2006) desarrollaron diversos estudios observacionales detallados, los cuales permitieron obtener resultados concernientes a la masa y distribución de tamaños para las diferentes clases dinámicas de la Región Transneptuniana. Recientemente, Kenyon et al. (2008) resumieron y discutieron las principales conclusiones derivadas a partir de aquellos estudios y analizaron la formación y evolución colisional de los objetos transneptunianos.

Aquí, nosotros presentamos el primer estudio destinado a describir la evolución colisional de los Plutinos, teniendo en cuenta las más importantes características dinámicas de la región resonante en la cual residen. Los principales propósitos de este trabajo son estimar la distribución de tamaños de los Plutinos, estudiar sus tiempos de vida colisionales medios, analizar la formación de familias, obtener tasas de eyección de fragmentos desde la resonancia 3:2 con Neptuno y discutir una posible conexión entre tales fragmentos y la población de Cometas Eclípticos

\subsection{Poblaciones Iniciales}

Con el objetivo de simular la evolución colisional y dinámica de los Plutinos, nuestro código numérico evoluciona en el tiempo el número de cuerpos residiendo en un conjunto de 
135 bines de tamaño, cuyos valores centrales se encuentran en el rango que va desde $D_{1}=$ $10^{-10} \mathrm{~km}$ a $D_{135}=2816.1 \mathrm{~km}$ de diámetro en una forma tal que desde un bin al siguiente, la masa de los cuerpos cambia en un factor 2 y el diámetro se modifica en un factor $2^{1 / 3}$. Para nuestras simulaciones, adoptamos una densidad de $1 \mathrm{~g} \mathrm{~cm}^{-3}$, la cual es consistente con los valores discutidos en la Sección 3.2.3, concerniente a los objetos de la Región Transneptuniana.

Durante los últimos años, diversos estudios observacionales han contribuido significativamente a nuestro conocimiento de la distribución de tamaños de la población de objetos transneptunianos y sus diferentes clases dinámicas. Contando con una muestra de 129 objetos, Bernstein et al. (2004) analizaron la distribución de magnitudes aparentes de la población de objetos transneptunianos en el rango $19.5 \leq m_{\mathrm{R}} \leq 28$.0. Estos autores sugirieron la existencia de un quiebre a un valor $m_{\mathrm{R}}=26$, proponiendo que una ley de potencia con dos pendientes sería adecuada para modelar dicha distribución. Por otra parte, Bernstein et al. (2004) dividieron la muestra de objetos con el fin de analizar las distribuciones de magnitudes asociadas a la población clásica y excitada de la Región Transneptuniana, estando esta última constituida por objetos resonantes y del Disco Dispersado. Similarmente a lo obtenido trabajando con la muestra completa, sus resultados indican la existencia de un quiebre cercano a $m_{\mathrm{R}}=23$ en dichas distribuciones individuales. Tiempo después, Elliot et al. (2005) analizaron las distribuciones de magnitudes de la población transneptuniana y sus diferentes clases dinámicas para objetos brillantes, con $m_{\mathrm{R}} \lesssim 24$. Estos autores derivaron resultados consistentes con aquellos previamente obtenidos por Bernstein et al. (2004) en ese rango de magnitudes. Más tarde, Petit et al. (2006) estudiaron la distribución de magnitudes de los objetos transneptunianos en el rango $22 \leq m_{\mathrm{R}} \leq 25$. Contrariamente a los derivado por Bernstein et al. (2004), estos autores propusieron que no hay evidencias que confirmen la existencia de un quiebre en dicha distribución, indicando que una ley de potencias con una única pendiente, ajusta perfectamente sus datos.

Recientemente, Kenyon et al. (2008) usaron la base de datos del MPC ${ }^{1}$ (Minor Planet Center) y los principales resultados de Bernstein et al. (2004), Elliot et al. (2005) y Petit et al. (2006), encontrando claras evidencias que sugieren diferencias físicas entre las distintas clases dinámicas de la Región Transneptuniana. Particularmente, sus análisis indican que la distribución de tamaños acumulada de la población resonante muestra un quiebre a un diámetro $D \sim 40-80 \mathrm{~km}$, lo cual está de acuerdo con aquello propuesto por Bernstein et al. (2004). Además, para objetos resonantes mayores, la población parece tener una distribución de tamaños acumulada con un exponente cuyo valor es $\sim 3$. Por otra parte, Kenyon et al. (2008) sugirieron que la población resonante contiene $\sim 0.01-0.05 M_{\oplus}$ en objetos con $D \gtrsim$ $20-40 \mathrm{~km}$.

Asumiendo un límite inferior de $0.01 M_{\oplus}$ para la masa contenida en objetos con $D \gtrsim$ 20-40 km, y considerando que la distribución de tamaños acumulada tiene un exponente cuyo valor es $\sim 3$ para cuerpos con $D \gtrsim 40-80 \mathrm{~km}$, obtenemos un único objeto del tamaño de Plutón en la población resonante de la Región Transneptuniana. En efecto, este objeto no es otro que Plutón mismo, el cual, como ya dijimos, se encuentra en la resonancia de movimientos medios 3:2 con Neptuno. Sin embargo, considerando el límite superior de 0.05 $M_{\oplus}$ para la masa contenida en objetos resonantes con $D \gtrsim 20-40 \mathrm{~km}$, es posible inferir la existencia de 5 objetos del tamaño de Plutón en la población resonante completa. Si estos 5 objetos estuviesen todos en la resonancia 3:2 con Neptuno, tendríamos un límite superior

\footnotetext{
${ }^{1}$ http://cfa-www.harvard.edu/iau/lists/MPLists.html
} 


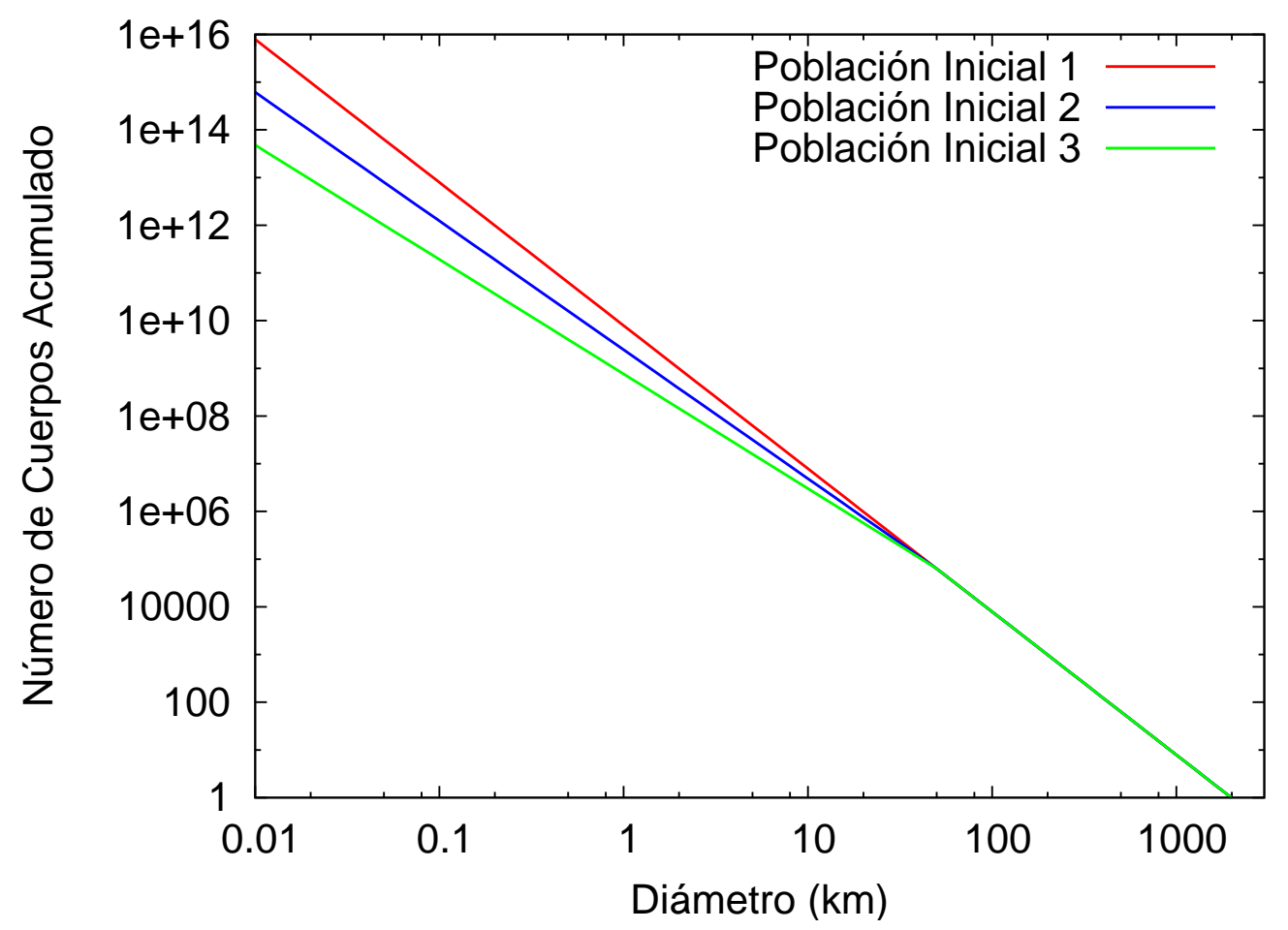

Figura 7.1. Poblaciones iniciales del modelo. Notemos que la Población Inicial 1 no muestra un quiebre, en acuerdo con aquello sugerido por Petit et al. (2006). Por otra parte, las Poblaciones Iniciales 2 y 3 son modeladas a partir de una ley de potencias con dos pendientes, siendo consistente con aquello discutido por Bernstein et al. (2004) y Kenyon et al. (2008).

para los grandes objetos de dicha región resonante. A partir de los estudios ya mencionados, esta resulta ser una posibilidad real, y más allá de que sólo Plutón haya sido observado hasta la fecha en la resonancia 3:2, la misma podría contener algunos objetos más con tamaños similares a este, que aun no han sido descubiertos. En efecto, Brown (2008) argumentó que entre dos o tres objetos grandes deben estar residiendo en la Región Transneptuniana sin haber sido observados aun. Nosotros consideraremos el límite de 5 objetos del tamaño de Plutón en la resonancia 3:2 sólo como una cota superior para nuestros cálculos (Sección 7.6.4). A continuación, nuestra discusión es desarrollada asumiendo la existencia de un único objeto del tamaño de Plutón en la resonancia 3:2 con Neptuno.

Con el objetivo de tener en cuenta la posible existencia de un quiebre en la población resonante, la distribución de tamaños acumulada inicial para $D \gtrsim 60 \mathrm{~km}$ es modelada a través de una ley de potencia con un exponente igual a 3 , mientras que para $D \lesssim 60 \mathrm{~km}$, la ley de potencia sigue un exponente $p$ con el objetivo de reproducir una dada masa inicial. A partir de esto, la forma general de la población acumulada inicial usada en nuestro modelo para estudiar la evolución colisional y dinámica de los Plutinos puede ser escrita como

$$
\begin{array}{lll}
N(>D)=C\left(\frac{1 \mathrm{~km}}{D}\right)^{p} & \text { para } & D \leq 60 \mathrm{~km}, \\
N(>D)=7,9 \times 10^{9}\left(\frac{1 \mathrm{~km}}{D}\right)^{3} & \text { para } & D>60 \mathrm{~km},
\end{array}
$$


donde $C=7.9 \times 10^{9}(60)^{p-3}$, por continuidad para $D=60 \mathrm{~km}$. Dada la incerteza en los parámetros de la distribución de tamaños de los Plutinos a pequeños diámetros, decidimos usar en el modelo tres poblaciones iniciales diferentes, las cuales son definidas como se muestra a continuación:

- Población Inicial 1, la cual adopta un exponente $p$ igual a 3 para $D \leq 60 \mathrm{~km}$, llevando $\mathrm{a} \sim 8.6 \times 10^{9}$ objetos con diámetros mayores a $1 \mathrm{~km}$,

- Población Inicial 2, la cual adopta un exponente $p$ igual a 2.7 para $D \leq 60 \mathrm{~km}$, llevando $\mathrm{a} \sim 2.7 \times 10^{9}$ objetos con diámetros mayores a $1 \mathrm{~km}, \mathrm{y}$

- Población Inicial 3, la cual adopta un exponente $p$ igual a 2.4 para $D \leq 60 \mathrm{~km}$, llevando $\mathrm{a} \sim 8.2 \times 10^{8}$ objetos con diámetros mayores a $1 \mathrm{~km}$.

Estas distribuciones de tamaños iniciales son mostradas en la Figura 7.1. Notemos que la Población Inicial 1 no muestra un quiebre, en acuerdo con aquello sugerido por Petit et al. (2006). Por otra parte, las Poblaciones Iniciales 2 y 3 son modeladas a partir de una ley de potencias de dos pendientes, siendo consistente con aquello discutido por Bernstein et al. (2004) y Kenyon et al. (2008).

En la Sección 7.6, discutimos la dependencia de nuestras simulaciones sobre la población inicial. Particularmente, en la Sección 7.6.4, estudiamos la sensibilidad de nuestros resultados al número de objetos del tamaño de Plutón en la resonancia 3:2 con Neptuno.

\subsection{Tratamiento Dinámico}

En el Capítulo 6, concerniente a los Troyanos jovianos, nuestro tratamiento dinámico fue desarrollado asumiendo que las posiciones ocupadas por estos objetos en el espacio de los elementos orbitales representan zonas estables de la resonancia 1:1 con Júpiter. Para los Troyanos jovianos de $L_{4}$, esta suposición resulta ser factible ya que contamos con una muestra estadísticamente significativa de 1155 objetos asociados a esta población. Sin embargo, para el caso particular de los Plutinos, este procedimiento no puede ser desarrollado. En efecto, hasta la fecha sólo se conocen $\sim 200$ objetos pertenecientes a la resonancia 3:2 con Neptuno, los cuales no ofrecen una estadística confiable para garantizar aquella suposición.

Con el objetivo de estudiar el espacio orbital ocupado en la resonancia 3:2 con Neptuno, desarrollamos una integración numérica de 197 candidatos a Plutinos extraídos de la base de datos del $\mathrm{MPC}^{2}$ (Minor Planet Center) con semiejes mayores entre 39 y 40 UA. Para hacer esto, asumimos que estos objetos son partículas sin masa sujetas a la influencia gravitacional del Sol (incluyendo las masas de los planetas terrestres) y de los cuatro planetas gigantes. La simulación es desarrollada con el código simpléctico EVORB de Fernández et al. (2002), usando un paso temporal de integración de 0.25 años. La evolución de las partículas de prueba es seguida sobre una escala de tiempo de $10^{7}$ años, la cual resulta ser mayor que cualquier período secular encontrado en esta resonancia (Morbidelli 1997). A partir de esta evolución, construimos nichos tridimensionales dentro de los límites de la resonancia 3:2 con anchos de $0.02 \mathrm{UA}, 0.0125$ y $1.125^{\circ}$ en semieje mayor $a$, excentricidad $e$, e inclinación $i$, respectivamente.

\footnotetext{
${ }^{2}$ http://www.cfa.harvard.edu/iau/lists/TNOs.html
} 

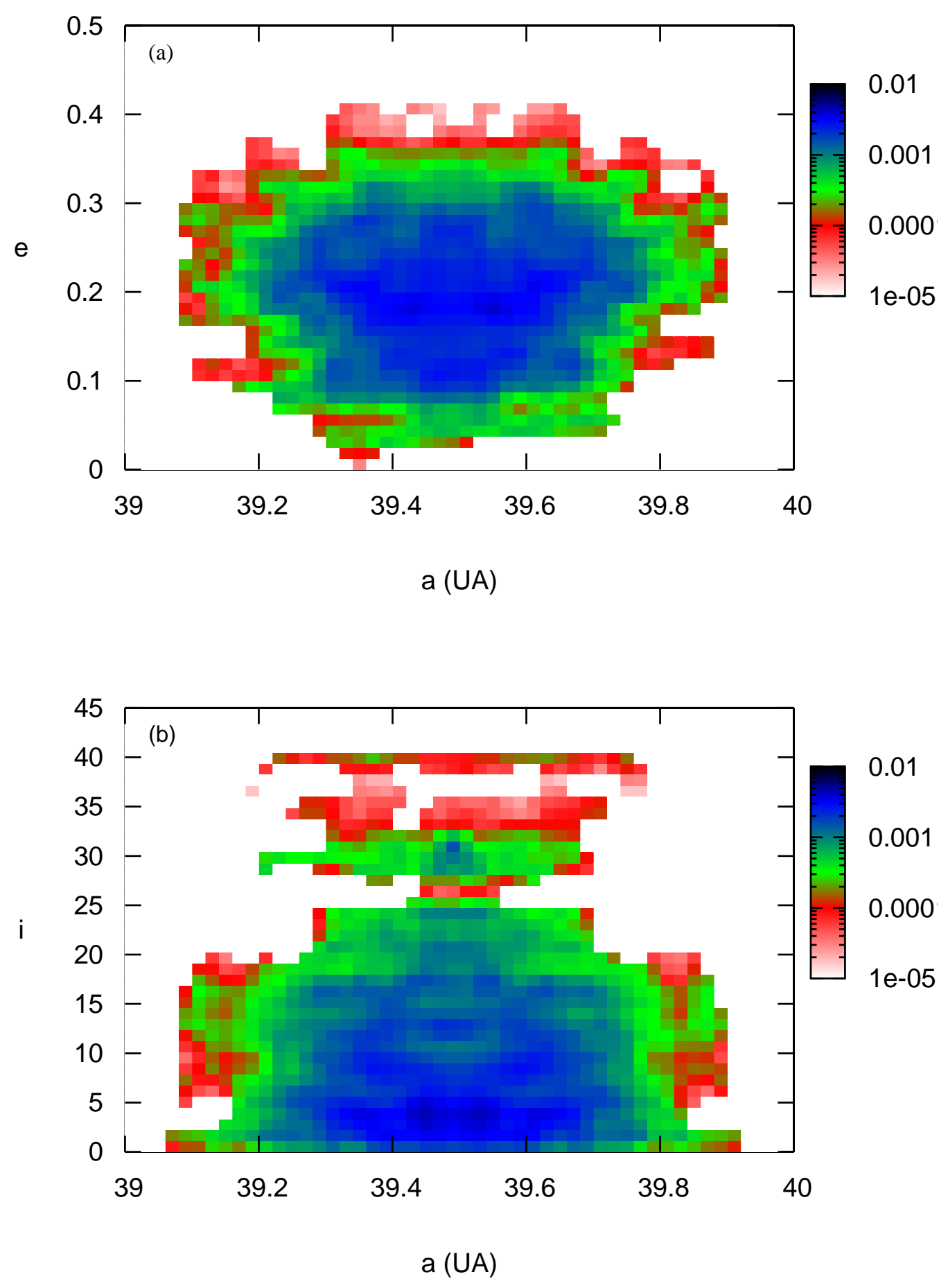

Figura 7.2. Distribución normalizada obtenida a partir de la evolución dinámica de 197 candidatos a Plutinos extraídos de la base de datos del MPC (Minor Planet Center) con semiejes mayores entre 39 y $40 \mathrm{UA}$, en los planos $(a, e) \mathrm{a})$ y $(a, i) \mathrm{b})$. La escala de color representa la densidad de Plutinos. Los colores azulados indican las regiones estadísticamente más probables donde los Plutinos pasan su tiempo. Figura realizada por la Dra. Romina P. Di Sisto. 
En cada nicho, calculamos el tiempo durante el cual cada uno es ocupado y lo normalizamos al tiempo total durante el cual todos los Plutinos pasan dentro de los límites de la resonancia. Este proceso nos permite construir un mapa de la distribución de Plutinos en los planos de elementos orbitales $(a, e)$ y $(a, i)$. Dichos mapas son mostrados en las Figuras 7.2a y b. Tales gráficos indican las regiones donde los Plutinos pasan su tiempo. Nosotros asumimos que las zonas de color en aquellos mapas son regiones con diferentes grados de probabilidad para que un Plutino sea encontrado, y ellas serán reconocidas bajo la denominación de "nichos de estabilidad" a lo largo de este trabajo. Por el contrario, las zonas vacías en aquellas figuras son regiones donde un Plutino no podría sobrevivir por un largo tiempo. Dichas regiones serán reconocidas bajo la denominación de "nichos de inestabilidad" a lo largo de nuestro análisis. Estas regiones corresponden a zonas inestables donde los objetos son rápidamente eyectados desde la resonancia, perdiendo el mecanismo de protección y encontrando a Neptuno en una escala de tiempo menor a $10^{7}$ años (Morbidelli 1997; Nesvorný \& Roig 2000).

La Sección 7.5 describe cómo los nichos de estabilidad e inestabilidad mostrados en la Figura 7.2 son incluidos en nuestro algoritmo con el objetivo de modelar el tratamiento dinámico del código. Por otra parte, en la Sección 7.6.5 discutimos la sensibilidad de nuestros resultados a la forma en la cual aquellos nichos son construidos así como también la dependencia de nuestras simulaciones sobre la distribución inicial de elementos orbitales de la población.

\subsection{Parámetros Colisionales}

\subsubsection{Probabilidad Intrínseca de Colisión y Velocidad de Impacto}

En este trabajo, adoptamos los valores medios de la probabilidad intrínseca de colisión $\left\langle P_{\mathrm{i}}\right\rangle$ y la velocidad de impacto $\langle V\rangle$ para Plutinos derivados a partir del algoritmo desarrollado por Dell'Oro et al. (2001), el cual tiene en cuenta el comportamiento resonante de los cuerpos. Basado en una muestra de 46 Plutinos y teniendo en cuenta la libración del argumento resonante $\sigma=2 \lambda_{\mathrm{N}}-3 \lambda-\tilde{\omega}$ alrededor de $180^{\circ}$, Dell'Oro et al. (2001) computaron valores de $\left\langle P_{\mathrm{i}}\right\rangle$ y $\langle V\rangle$ de $4.44 \pm 0.04 \times 10^{-22} \mathrm{~km}^{-2}$ año ${ }^{-1}$ y $1.44 \pm 0.71 \mathrm{~km} \mathrm{~s}^{-1}$, respectivamente.

\subsubsection{Leyes de Impacto}

De acuerdo a los argumentos presentados en la Sección 3.3, decidimos seleccionar como parámetros de entrada de nuestro modelo colisional una combinación de $Q_{\mathrm{S}}$ y $f_{\text {ke }}$ que produzcan la ley $Q_{\mathrm{D}}$ de Benz \& Asphaug (1999) para cuerpos helados a una velocidad de 3 $\mathrm{km} \mathrm{s}^{-1}$.

En el Capítulo 6, concerniente al estudio de los asteroides Troyanos jovianos de $L_{4}$, analizamos la dependencia de nuestras simulaciones numéricas sobre la energía específica de fragmentación $Q_{\mathrm{S}}$. Nuestro trabajo indica que cuanto más pequeño es el gap entre $Q_{\mathrm{S}}$ y $Q_{\mathrm{D}}$, mayor es la tasa de eyección de fragmentos colisionales desde la resonancia. Además, aquel estudio también nos permite inferir que la formación de familias resulta ser más efectiva en aquellas simulaciones que usan un gap chico entre las leyes $Q_{\mathrm{S}}$ y $Q_{\mathrm{D}}$. Siguiendo estos argumentos, decidimos desarrollar nuestra investigación de la población de Plutinos usando una única ley $Q_{\mathrm{S}}$ con un pequeño gap respecto de la ley $Q_{\mathrm{D}}$ de Benz \& Asphaug (1999) para cuerpos helados a $3 \mathrm{~km} \mathrm{~s}^{-1}$. Este procedimiento nos permite obtener una cota superior a la 


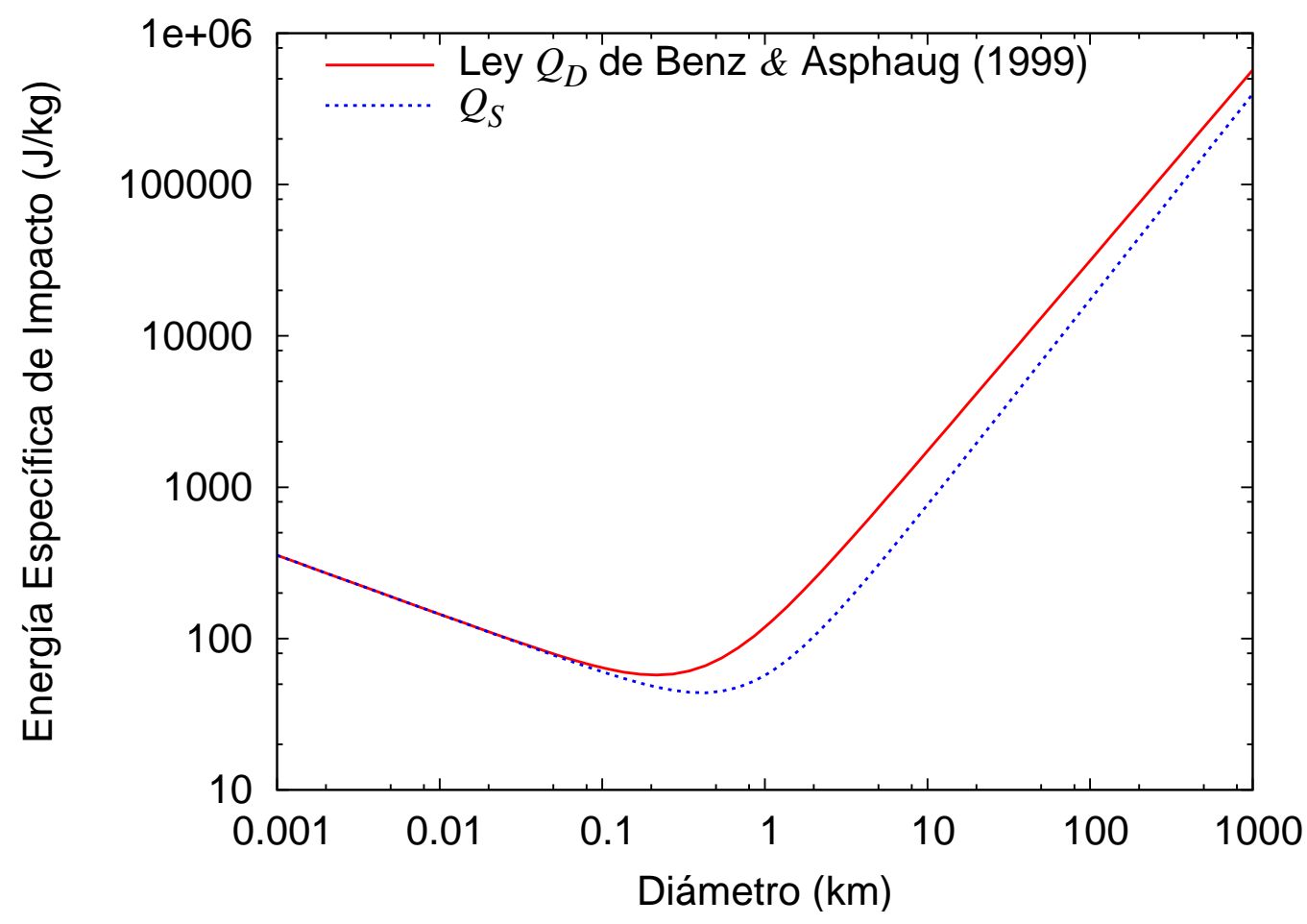

Figura 7.3. Leyes de impacto. La curva punteada azul representa la ley $Q_{\mathrm{S}}$ usada en nuestras simulaciones, mientras que la ley $Q_{\mathrm{D}}$ de Benz \& Asphaug (1999) para cuerpos helados a una velocidad de $3 \mathrm{~km} \mathrm{~s}^{-1}$ es ilustrada a través de la curva roja.

tasa de eyección de fragmentos colisionales desde la resonancia 3:2 con Neptuno y maximizar el número de familias que podrían ser encontradas en esta región resonante.

La ley $Q_{\mathrm{S}}$ usada en nuestras simulaciones es ilustrada en la Figura 7.3 a través de la curva punteada azul, y puede ser representada a partir de una expresión de la forma

$$
Q_{\mathrm{S}}=C_{1} D^{-\lambda_{1}}\left(1+\left(C_{2} D\right)^{\lambda_{2}}\right),
$$

donde $C_{1}, C_{2}, \lambda_{1}$, y $\lambda_{2}$ son coeficientes constantes cuyos valores son 24, 1.2, 0.39 y 1.75, respectivamente ${ }^{3}$. Por otra parte, la curva roja de la Figura 7.3 representa la ley $Q_{\mathrm{D}}$ de Benz \& Asphaug (1999) para cuerpos helados a $3 \mathrm{~km} \mathrm{~s}^{-1}$.

Una vez que la ley $Q_{\mathrm{S}}$ es especificada, es necesario ajustar el parámetro de ineslaticidad $f_{\text {ke }}$ para obtener la ley $Q_{\mathrm{D}}$ de Benz \& Asphaug (1999). De acuerdo a lo discutido en la Sección $4.4, f_{\mathrm{ke}}$ adopta una expresión de la forma

$$
f_{\mathrm{ke}}=f_{\mathrm{ke}_{0}}\left(\frac{D}{1000 \mathrm{~km}}\right)^{\gamma}
$$

Nuestras simulaciones indican que la ley $Q_{\mathrm{D}}$ de Benz \& Asphaug (1999) para cuerpos helados a $3 \mathrm{~km} \mathrm{~s}^{-1}$ es obtenida con buena precisión a partir de la combinación de la ley $Q_{\mathrm{S}}$ seleccionada y $f_{\mathrm{ke}}$, con $f_{\mathrm{ke}_{0}}=0.27$ y $\gamma=0.7$. Tales valores son consistentes con aquellos discutidos por Davis et al. (1989).

\footnotetext{
${ }^{3}$ Notemos que esta ley $Q_{\mathrm{S}}$ corresponde a la $Q_{\mathrm{S}, 1}$ de la Figura $6.4 \mathrm{~b}$, la cual maximiza la tasa de eyección de fragmentos colisionales y la formación de familias para la población de Troyanos jovianos.
} 


\subsection{Modelo Dinámico y Colisional}

En esta sección presentamos el modelo dinámico y colisional usado para estudiar la evolución de los Plutinos.

Similarmente a lo realizado en los Capítulos 5 y 6 , nuestro algoritmo no evoluciona en el tiempo los primeros 60 bines de tamaño, cuyos diámetros centrales van desde $10^{-10}$ a $10^{-4} \mathrm{~km}$, de acuerdo a aquello sugerido por Campo Bagatin et al. (1994a) y Campo Bagatin (1998). En efecto, esta parte de la población es usada únicamente a modo de proyectiles. Posteriormente, su distribución de tamaños es determinada en cada paso de tiempo, extrapolando la pendiente de la distribución asociada a los 10 bines de tamaño siguientes.

El modelo numérico usado en este estudio es equivalente a aquel descripto en la Sección 5.6. Al igual que para el caso de los Troyanos jovianos de $L_{4}$, el código toma una forma simple ya que sólo se involucra a los Plutinos como única población del modelo.

Supongamos que analizamos una colisión entre dos Plutinos $o$ y $o^{\prime}$ con diámetros $D_{o} \mathrm{y}$ $D_{o^{\prime}}$, respectivamente. Las distribuciones de fragmentos y velocidades, y la velocidad de escape del sistema de cuerpos colisionantes, son calculadas con el modelo colisional descripto en el Capítulo 4 y de la forma detallada en la Sección 5.6. Para determinar el destino final de los fragmentos que escapan del sistema de cuerpos colisionantes, proponemos un mecanismo simple en el cual se tienen en cuenta los resultados de la integración numérica de Plutinos descripta en la Sección 7.3. Como paso inicial a realizar en cada intervalo de tiempo, una órbita característica es generada al azar para cada colisión entre Plutinos de diámetros $D_{o}$ y $D_{o^{\prime}}$ en la resonancia 3:2 con Neptuno. A partir de las Figuras 7.2a y b, normalizamos la distribución de Plutinos en cada nicho previamente definido en la Sección 7.3, la cual es equivalente a una función de distribución de probabilidades $f(a, e, i)$. Luego, asignamos elementos orbitales $a, e$, e $i$ a nuestros Plutinos ficticios iniciales, siguiendo el método de aceptación-rechazo de John von Neumann (Knuth 1981). Tal como expresamos en la Sección 6.5, esta técnica indica que si un conjunto de números $a^{*}, e^{*}$, e $i^{*}$ es seleccionado al azar desde el dominio de la función $f$ (esto es, $a^{*}, e^{*}$, e $i^{*}$ entre 39 y $40 \mathrm{UA}, 0$ y 0.5, y 0 y $45^{\circ}$, respectivamente), y otro conjunto de números $f^{*}$ es dado aleatoriamente desde el rango de la función (esto es, $f^{*}$ entre 0 y 0.0012$)$, entonces la condición $f^{*} \leq f\left(a^{*}, e^{*}, i^{*}\right)$ generará una distribución para $\left(a^{*}, e^{*}, i^{*}\right)$ cuya densidad es $f\left(a^{*}, e^{*}, i^{*}\right) d a^{*} d e^{*} d i^{*}$. Tales valores $\left(a^{*}, e^{*}, i^{*}\right)$ serán aceptados como posibles condiciones iniciales para el semieje mayor, excentricidad e inclinación de los Plutinos, en acuerdo con los datos observacionales y la integración numérica desarrollada en la Sección 7.3. Al igual que en el estudio de los Troyanos jovianos de $L_{4}$, la evolución de $a, e$ e $i$ es desarrollada asumiendo a las mismas como variables no correlacionadas, a pesar de que en una resonancia de movimientos medios la evolución de estos elementos está acoplada. Nuevamente, destacamos que un tratamiento más riguroso sería muy dificultoso, y creemos que los resultados no serían demasiado diferentes a aquellos encontrados aquí. Finalmente, dada la longitud del nodo ascendente $\Omega$, el argumento del perihelio $\omega$ y la anomalía media $M$ entre 0 y $360^{\circ}$, una órbita puede ser asignada y a partir de esto, un par posición-velocidad puede ser derivado para cada uno de los Plutinos colisionantes.

Para determinar el destino final de los fragmentos que escapan, es necesario calcular cuáles son sus elementos orbitales una vez que ellos son eyectados con una dada velocidad respecto del cuerpo que los originó. Inmediatamente antes de la colisión, asumimos que la posición y velocidad baricéntricas de los fragmentos son iguales a aquellas asociadas a su cuerpo 
precursor. Inmediatamente después de la colisión, consideramos que la posición baricéntrica de los fragmentos es equivalente a aquella asociada a su cuerpo precursor, mientras que la velocidad relativa de los mismos con respecto a este último es igualmente particionada entre las tres componentes cartesianas. Una vez que obtenemos la posición y velocidad baricéntricas de los fragmentos posteriores al impacto, es posible calcular sus elementos orbitales y sus destinos finales. Para hacer esto, usamos los siguientes criterios:

1. Los fragmentos permanecen en la resonancia 3:2 si las combinaciones de valores $(a, e)$ y $(a, i)$ están asociadas con alguno de los nichos de estabilidad mostrados en las Figuras $7.2 \mathrm{a}$ y b, respectivamente.

2. Por el contrario, los fragmentos son eyectados de la resonancia 3:2, dejando de participar de la evolución colisional, si se satisface alguna de las siguientes condiciones:

- excentricidad $e \geq 1$,

- excentricidad $e<1$ con valores $(a, e, i)$ que exceden los límites de la resonancia 3:2 (Sección 7.3),

- excentricidad $e<1$ con valores $(a, e)$ y $(a, i)$ asociados con alguno de los nichos de inestabilidad mostrados en las Figuras 7.2a y b, respectivamente.

Bajo estas circunstancias, los fragmentos tienen dos posibilidades: permanecer en la población de Plutinos o escapar fuera de la resonancia. El número de fragmentos eyectados desde los cuerpos $o$ y $o^{\prime}$ que permanecen en la resonancia es guardado en los vectores $F_{o}(k)$ y $F_{o^{\prime}}(k)$, respectivamente. El número de fragmentos que no permanecen en la resonancia no debe ser guardado en estos vectores. Sin embargo, dicha información debe ser conservada ya que la misma resulta ser relevante para computar la tasa de eyección colisional desde dicha región en cada paso de tiempo.

La frecuencia de colisión entre Plutinos o y $o^{\prime}$, con diámetros $D_{o}$ y $D_{o^{\prime}}$, respectivamente, puede ser calculada a partir de la siguiente expresión

$$
f_{o, o^{\prime}}=\frac{\left\langle P_{\mathrm{i}}\right\rangle}{4}\left(D_{o}+D_{o^{\prime}}\right)^{2}
$$

donde $\left\langle P_{\mathrm{i}}\right\rangle$ es la probabilidad intrínseca de colisión asociada a la población de Plutinos (Sección 7.4.1), y tiene unidades de $\mathrm{km}^{-2}$ año ${ }^{-1}$. Si $\Delta t$ representa un dado intervalo y $N_{o, o^{\prime}}$ es el número de pares de Plutinos con diámetros $D_{o}$ y $D_{o^{\prime}}$, es posible obtener el número total de colisiones entre dichos objetos durante $\Delta t$ a partir de la expresión

$$
N_{\mathrm{col}}=f_{o, o^{\prime}} N_{o, o^{\prime}} \Delta t
$$

Sean $N_{o}$ y $N_{o^{\prime}}$ el número de Plutinos de diámetro $D_{o}$ y $D_{o^{\prime}}$, respectivamente. Tal como se indicó en la Sección 5.6, si los cuerpos colisionantes pertenecen al mismo bin de tamaño, esto es, si $o=o^{\prime}$, entonces $N_{o}=N_{o^{\prime}}$ y por ende, el número de pares de Plutinos con diámetros $D_{o}$ y $D_{o^{\prime}}$ está dado por

$$
N_{o, o^{\prime}}=\frac{N_{o}\left(N_{o^{\prime}}-1\right)}{2}
$$

Para todos los otros casos,

$$
N_{o, o^{\prime}}=N_{o} N_{o^{\prime}}
$$


A partir de esto, el número total de fragmentos asociados al bin de tamaño $k$ que permanecen en la resonancia 3:2 con Neptuno luego de ser eyectados de los cuerpos o como resultado de colisiones con cuerpos $o^{\prime}$, durante un intervalo $\Delta t$, está dado por $F_{o}(k) f_{o, o^{\prime}} N_{o, o^{\prime}} \Delta t$. Equivalentemente, el número total de fragmentos asociados al bin de tamaño $k$ que permanecen en la resonancia luego de ser eyectados de los cuerpos $o^{\prime}$ como resultado de colisiones con cuerpos $o$, durante un intervalo $\Delta t$, está dado por $F_{o^{\prime}}(k) f_{o, o^{\prime}} N_{o, o^{\prime}} \Delta t$. Si tenemos en cuenta las interacciones entre todos los objetos que constituyen la población de Plutinos, es posible derivar la variación en el número de cuerpos asociados a un dado bin de tamaño $k$, en cada paso de tiempo. Dichas variaciones son referidas como $\Delta N_{k}$.

Para estudiar la evolución en el tiempo de la población de Plutinos, el paso de tiempo $\Delta t$ es calculado en una forma tal que el cambio relativo en el número de objetos en cualquier bin de tamaño sea siempre menor que el $1 \%$.

\subsection{Resultados}

En esta sección, discutimos los resultados generales obtenidos a partir de una serie de simulaciones numéricas destinadas a estudiar la evolución colisional y dinámica de la población de Plutinos sobre $4.5 \times 10^{9}$ años.

\subsubsection{Distribución de Tamaños de los Plutinos}

La Figura 7.4a muestra la distribución de tamaños acumulada de los Plutinos después de $4.5 \times 10^{9}$ años de evolución, obtenida a partir de la Población Inicial 1, la cual sigue una ley de potencias con un exponente igual a 3 para todos los tamaños. A partir de esto, encontramos que no es posible reproducir el quiebre sugerido por Bernstein et al. (2004) y Kenyon et al. (2008) a un diámetro $D \sim 40-80 \mathrm{~km}$, usando una ley de potencias con una única pendiente para describir la población inicial. Por el contrario, la población evolucionada final sólo muestra un quiebre a un diámetro $D \sim 2 \mathrm{~km}$, mientras que los objetos más grandes no son significativamente alterados por colisiones sobre la edad del Sistema Solar. En efecto, la curva roja en la Figura 7.5 representa nuestros resultados concernientes a los tiempos de vida colisionales medios de Plutinos obtenidos a partir de la Población Inicial 1. Esto indica que los Plutinos con diámetros $D \gtrsim 5 \mathrm{~km}$ tienen tiempos de vida medios superiores a la edad del Sistema Solar, lo cual implica que tales objetos probablemente han sobrevivido inalterados por impactos catastróficos sobre $4.5 \times 10^{9}$ años. La confirmación de la existencia de un quiebre a un diámetro $D \sim 40-80 \mathrm{~km}$ en la distribución de tamaños acumulada de los Plutinos debería descartar a una ley de potencias con una única pendiente como una posible población inicial.

Por otra parte, la Figura 7.4b muestra la distribución de tamaños acumulada de los Plutinos después de $4.5 \times 10^{9}$ años, usando la Población Inicial 2, la cual presenta un quiebre a un diámetro $D=60 \mathrm{~km}$ y sigue una ley de potencias con un exponente igual a 2.7 para tamaños menores. Nuestros resultados indican que la población evolucionada final produce un quiebre a un diámetro $D \sim 2 \mathrm{~km}$, mientras que los cuerpos más grandes no sufren una evolución colisional significativa sobre la edad del Sistema Solar, representando una población primordial. En efecto, la curva azul en la Figura 7.5 indica que los tiempos de vida colisionales medios de Plutinos con diámetros $D \gtrsim 2 \mathrm{~km}$ son superiores a la edad del Sistema Solar. De la misma forma, la Figura 7.4c presenta nuestros resultados concernientes a la distribución de tamaños 

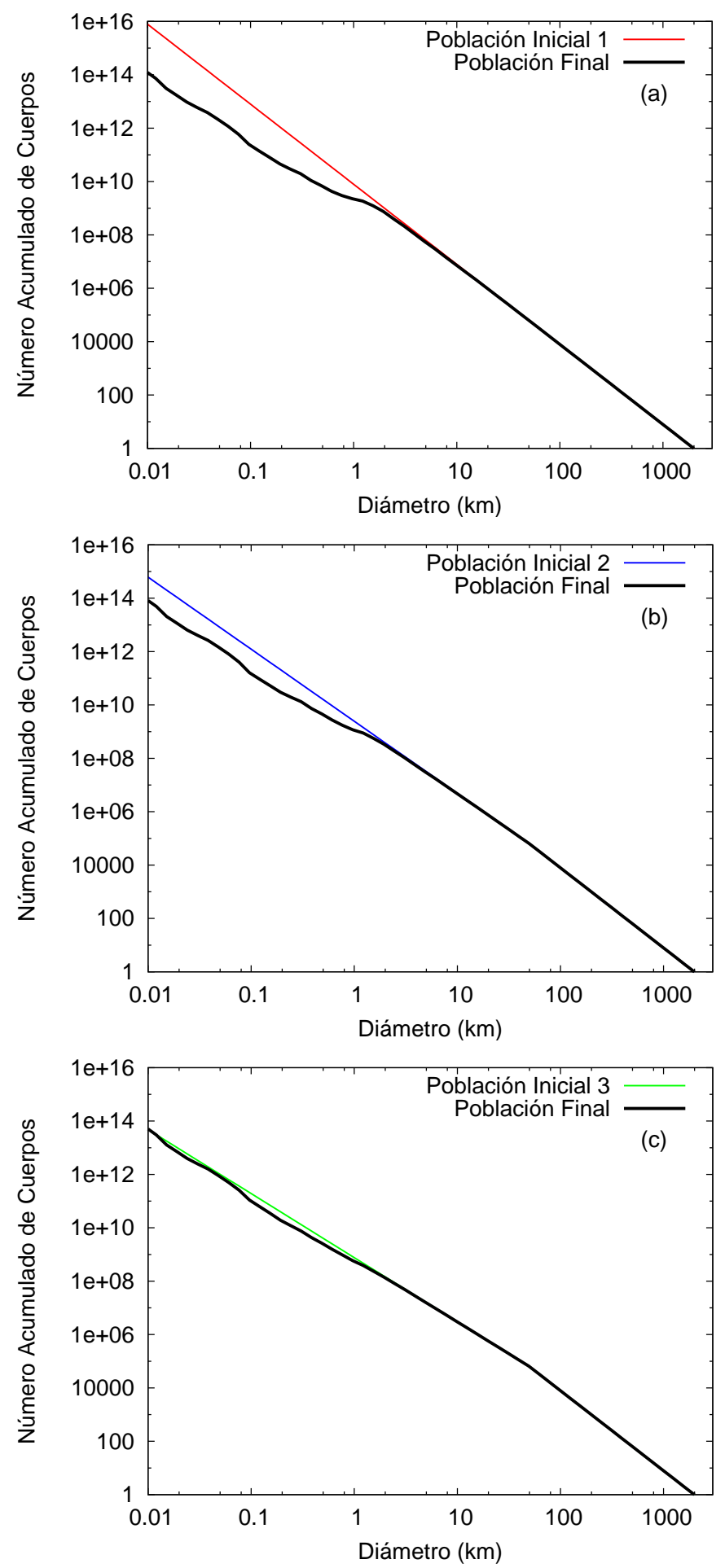

Figura 7.4. Distribuciones de tamaños acumuladas de los Plutinos después de $4.5 \times 10^{9}$ años de evolución, obtenidas a partir de las tres poblaciones iniciales propuestas en la Sección 7.2. Las mismas indican que el quiebre sugerido por Bernstein et al. (2004) y Kenyon et al. (2008) a un diámetro $D \sim 40-80 \mathrm{~km}$ no puede ser el resultado de la evolución colisional, y por ende debería ser una característica primordial de esta población. 


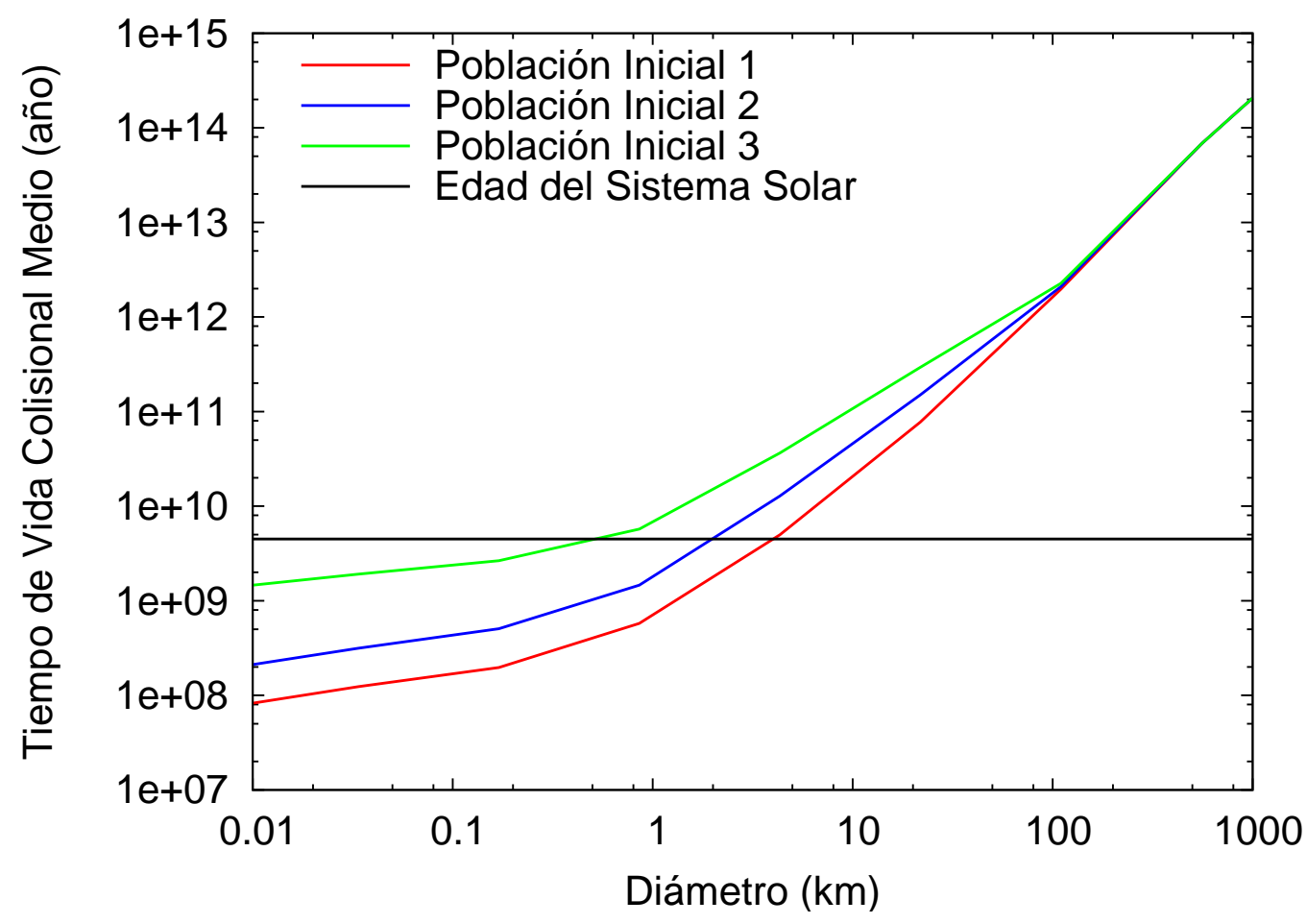

Figura 7.5. Tiempos de vida colisionales medios de Plutinos obtenidos usando las diferentes poblaciones iniciales definidas en la Sección 7.2. A partir de estos resultados, los Plutinos con diámetros mayores a unos pocos kilómetros tienen tiempos de vida colisionales medios superiores a la edad del Sistema Solar, la cual está representada por la línea horizontal negra. De acuerdo con esto, tales objetos resultan ser primordiales y posiblemente han sobrevivido sin ser catastróficamente fragmentos sobre $4.5 \times 10^{9}$ años de evolución.

acumulada de los Plutinos después de $4.5 \times 10^{9}$ años, obtenida a partir de la Población Inicial 3 , la cual tiene un quiebre a un diámetro $D=60 \mathrm{~km}$ y sigue una ley de potencias de 2.4 para tamaños menores. En este caso, la población evolucionada final muestra un suave quiebre a un diámetro $D \sim 1 \mathrm{~km}$. Además, la curva verde en la Figura 7.5 nos permite inferir que los Plutinos con diámetros $D \gtrsim 1 \mathrm{~km}$ son primordiales ya que sus tiempos de viva colisionales medios son superiores a la edad del Sistema Solar.

Nuestras simulaciones indican que la existencia de un quiebre a un diámetro $D \sim 40-80$ $\mathrm{km}$ en la distribución de tamaños acumulada de los Plutinos debería ser un rasgo primordial de la población y no el resultado de la evolución colisional. Esto concuerda fuertemente con los resultados derivados por Charnoz \& Morbidelli (2007), quienes argumentaron que el quiebre a un diámetro $D \sim 50 \mathrm{~km}$ en la distribución de objetos del Cinturón de Kuiper debería ser primordial.

\subsubsection{Familias de Plutinos}

La existencia de familias en una dada región representa un resultado natural de la actividad colisional. De modo similar a lo realizado en los Capítulos 5 y 6, aquí, estudiamos la existencia de familias en la resonancia 3:2 con Neptuno formadas a partir de la fractura de cuerpos 


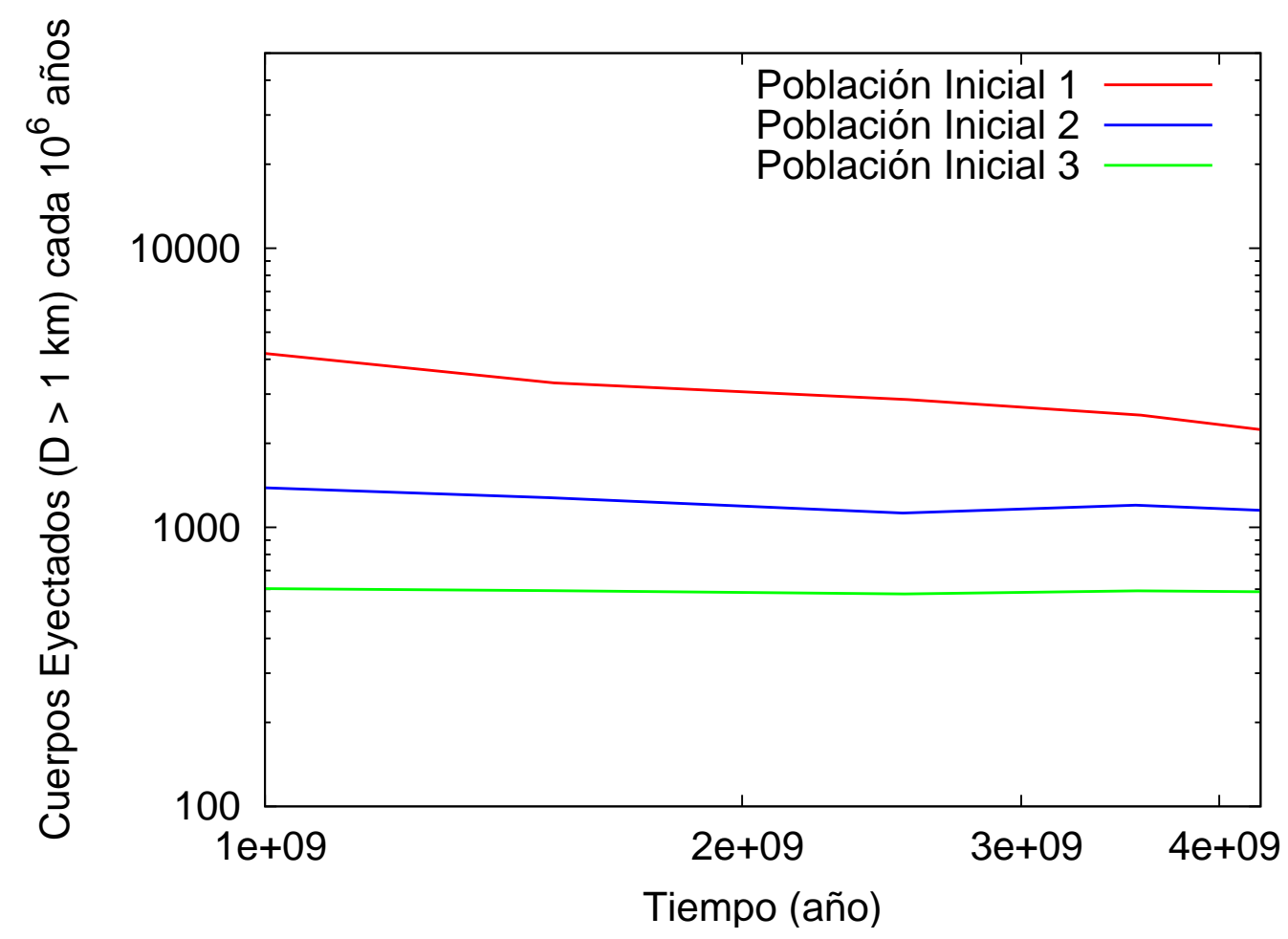

Figura 7.6. Tasas de eyección de fragmentos colisionales con diámetros $D>1 \mathrm{~km}$ por millón de años, desde la resonancia 3:2 con Neptuno, obtenidas a partir de las diferentes poblaciones iniciales definidas en la Sección 7.2. A partir de las mismas, determinamos que un objeto con un diámetro $D>1 \mathrm{~km}$ es eyectado cada $\sim 400-1700$ años desde esta región resonante.

precursores con diámetros $D \gtrsim 100 \mathrm{~km}$, que dispersen fragmentos con tamaños de $\sim 30-40$ $\mathrm{km}$.

Nuestras simulaciones indican que la formación de grandes familias de Plutinos no depende fuertemente de la población inicial. En efecto, a partir de cualquiera de las tres poblaciones iniciales propuestas en la Sección 7.2, 7 grandes familias son formadas en la resonancia de movimientos medios 3:2 con Neptuno sobre la edad del Sistema Solar.

\subsubsection{Tasas de Eyección desde la Resonancia 3:2 con Neptuno}

La Figura 7.6 muestra las tasas de eyección de fragmentos colisionales con diámetros $D>$ $1 \mathrm{~km}$ por millón de años, desde la resonancia 3:2 con Neptuno, como función del tiempo y sobre la edad del Sistema Solar. En la misma se aprecia las estimaciones obtenidas a partir de las diferentes poblaciones iniciales definidas en la Sección 7.2. Con el objetivo de estudiar la contribución de los Plutinos a la población actual de Cometas Eclípticos, estimamos una tasa media de eyección de fragmentos desde la resonancia 3:2 para cada una de nuestras simulaciones sobre los últimos $5 \times 10^{8}$ años de evolución, donde el número de cuerpos removidos por unidad de tiempo es más o menos constante y además la muestra de datos resulta ser estadísticamente significativa. Nuestros resultados indican que las tasas máximas de eyección desde la resonancia $3: 2$ son de $\sim 2.2 \times 10^{3}, 1.2 \times 10^{3}$ y $5.9 \times 10^{2}$ objetos con diámetros $D>1 \mathrm{~km}$ 
por millón de años, obtenidas a partir de las Poblaciones Iniciales 1,2 y 3, respectivamente. De este modo, esperaríamos un máximo de 1 Cometa Eclíptico con un diámetro $D>1 \mathrm{~km}$ cada 400-1 700 años desde la resonancia 3:2, mientras que las estimaciones de Di Sisto \& Brunini (2007) sugieren la inyección de 4 Centauros con una radio $R>1 \mathrm{~km}$ cada año desde el Disco Dispersado de la Región Transneptuniana.

En la Sección 7.5, discutimos diversos criterios para determinar el destino final de los fragmentos de Plutinos y a partir de esto calcular las tasas de eyección desde la resonancia de movimientos medios 3:2 con Neptuno. Nuestras simulaciones indican que la eyección de fragmentos desde la resonancia 3:2 es debida a variaciones en los parámetros orbitales $a, e$ e $i$, los cuales toman valores asociados a alguno de los nichos de inestabilidad mostrados en la Figura 7.2 o exceden los límites asumidos para esta región resonante (Sección 7.3). En estos casos, los fragmentos tienen excentricidades $e<1$ (Sección 7.5). En efecto, para todos nuestros experimentos numéricos, encontramos que más del $99 \%$ de los fragmentos que escapan desde la resonancia 3:2 son eyectados debido a los dos mecanismos anteriormente mencionados, descartando la eyección colisional hiperbólica como una fuente de remoción de Plutinos. La Figura 7.7 es un mapa normalizado representativo de los Plutinos eyectados desde la resonancia 3:2 con Neptuno, en función del semieje mayor, excentricidad e inclinación.

Por otra parte, para todos los casos, la mayoría de los cuerpos eyectados desde la resonancia de movimientos medios 3:2 con Neptuno con diámetros $D>1 \mathrm{~km}$ se distribuyen en el rango de $1<D<5 \mathrm{~km}$. En efecto, nuestros resultados indican que la tasa de eyección de fragmentos con diámetros $D>5 \mathrm{~km}$ resulta ser varios órdenes de magnitud menor que aquella asociada a fragmentos con diámetros $D>1 \mathrm{~km}$. Además, es interesante notar que los objetos eyectados desde la resonancia son fragmentos resultantes de impactos entre cuerpos precursores con diámetros en el rango $10<D<200 \mathrm{~km}$.

\subsubsection{Sensibilidad al Número de Objetos del Tamaño de Plutón}

Los resultados discutidos hasta aquí han sido derivados asumiendo la existencia de un único objeto del tamaño de Plutón en la resonancia 3:2 con Neptuno. Como indicamos en la Sección 7.2, si consideramos el límite superior de $0.05 M_{\oplus}$ sugerido por Kenyon et al. (2008) para la masa de la población resonante en objetos con diámetros $D \gtrsim 20-40 \mathrm{~km}$, entonces 5 objetos del tamaño de Plutón deberían ser encontrados en toda la población resonante, de acuerdo a los parámetros para la distribución de tamaños de tal población, derivados por Bernstein et al. (2004) y Kenyon et al. (2008).

Para testear la dependencia de nuestros resultados sobre el número de objetos del tamaño de Plutón, llevamos a cabo diversos experimentos numéricos considerando "5 Plutones" en la resonancia 3:2 con Neptuno. Este número es seguramente una sobreestimación (Sección 7.2); sin embargo, nuestro propósito es sólo ilustrar el efecto de la masa total en la población sobre la historia colisional de los Plutinos.

Nuestros resultados sugieren que cuanto mayor es el número de objetos del tamaño de Plutón en la resonancia 3:2 con Neptuno, mayor es la tasa de eyección de fragmentos desde aquella región resonante y el número de familias formadas sobre $4.5 \times 10^{9}$ años. En efecto, si asumimos que 5 "Plutones" están presentes en la resonancia 3:2, un cuerpo con un diámetro $D>1 \mathrm{~km}$ es eyectado cada algunas decenas de años, mientras que $\sim 170$ grandes familias son formadas sobre la edad del Sistema Solar. De acuerdo a las estimaciones de Di Sisto \& 

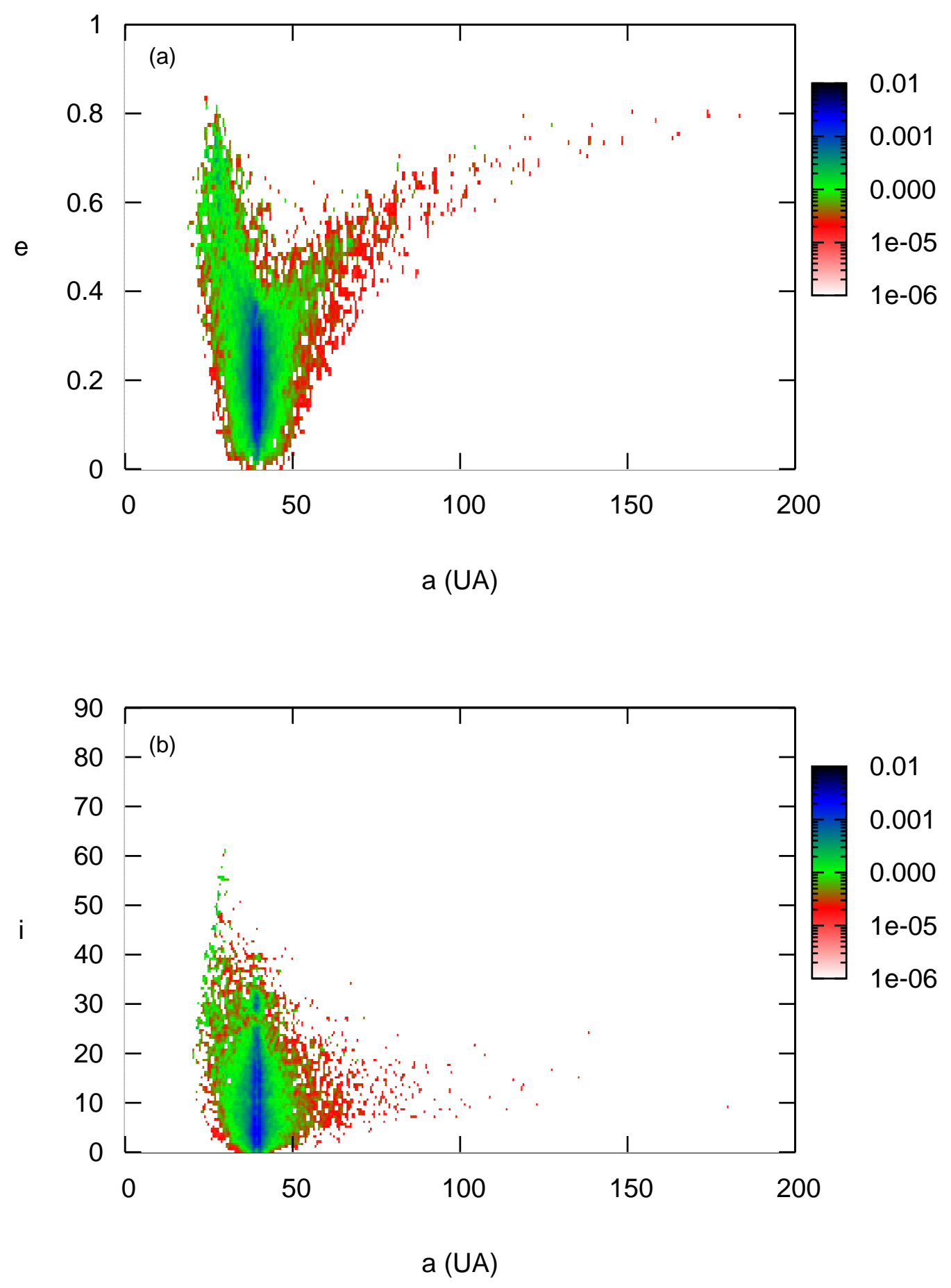

Figura 7.7. Distribución normalizada de los Plutinos eyectados desde la resonancia 3:2 con Neptuno en función del semieje mayor, excentricidad e inclinación. Figura realizada por la Dra. Romina P. Di Sisto. 
Brunini (2007), concluimos que es necesario especificar el número de objetos del tamaño de Plutón existentes en la resonancia 3:2 con Neptuno para determinar si esta región resonante puede ser considerada una fuente importante de Cometas Eclípticos.

\subsubsection{Robustez de los Resultados}

Los resultados mostrados en la Sección 7.6 han sido obtenidos usando los nichos de estabilidad e inestabilidad definidos en la Sección 7.3, los cuales presentan anchos de 0.02 UA, 0.0125 y $1.125^{\circ}$ en semieje mayor $a$, excentricidad $e$ e inclinación $i$, respectivamente. Con el objetivo de testear la dependencia de nuestros resultados sobre el tamaño de aquellos nichos, llevamos a cabo diversos experimentos numéricos incrementando los anchos de tales regiones en $a, e$ e $i$, lo cual lleva a magnificar la región de estabilidad. En términos generales, cuanto más grande es el área de los nichos, más pequeña es la tasa de eyección de fragmentos desde la resonancia de movimientos medios 3:2 con Neptuno. En este estudio, seleccionamos nichos pequeños con el objetivo de minimizar la influencia de las regiones de más baja densidad mostradas en la Figura 7.2. Por otra parte, encontramos que los resultados concernientes a la distribución de tamaños de los Plutinos y la formación de familias no son sensibles al tamaño de las regiones de estabilidad e inestabilidad construidas para desarrollar nuestro tratamiento dinámico.

De la misma forma, también desarrollamos algunas simulaciones numéricas con el objetivo de explorar la sensibilidad de nuestros resultados a la masa inicial de la población. En efecto, los estudios desarrollados por Stern (1996), Stern \& Colwell (1997a) y Stern \& Colwell (1997b) sugieren que la masa primordial de la Región Transneptuniana entre 30 y 50 UA fue probablemente del orden de 10 a $50 M_{\oplus}$, lo cual es $\sim 100$ veces su valor actual. Además, estos autores indicaron que una vez que Neptuno alcanzó una cierta fracción de su masa final, el disco llegó a ser altamente erosivo para objetos con radios $R \lesssim 20-30 \mathrm{~km}$, llevando a una rápida remoción de masa sobre una escala de tiempo de $\sim 10^{8}$ años. Para tener en cuenta estos resultados y siguiendo la idea propuesta por O'Brien \& Greenberg (2005), llevamos a cabo diversos experimentos numéricos incluyendo un breve período de evolución primordial al comienzo de la simulación. Para hacer esto, cada una de las poblaciones iniciales definidas en la Sección 7.2 es multiplicada por una factor igual a 100 y luego, su evolución es seguida por $10^{8}$ años. Una vez que esta fase de evolución primordial finaliza, la población residual correspondiente es reducida por el mismo factor y finalmente su evolución es analizada por el resto de los $4.5 \times 10^{9}$ años. Nuestros resultados sugieren que la distribución de tamaños de los Plutinos, sus tiempos de vida colisionales medios, la tasa de eyección de fragmentos desde la resonancia 3:2 con Neptuno y la formación de familias no muestran cambios relevantes cuando este período de evolución primordial es incluido en el modelo.

Finalmente, vale la pena recordar que los resultados presentados en este estudio han sido derivados generando valores iniciales del semieje mayor $a$, excentricidad $e$ e inclinación $i$ a partir de la evolución dinámica de 197 Plutinos de prueba mostrada en la Figura 7.2. Con el objetivo de testear la dependencia de nuestros resultados sobre la distribución orbital inicial, nosotros desarrollamos diversas simulaciones comenzando con una población dinámicamente fría, con excentricidades e inclinaciones menores a 0.05 y $10^{\circ}$, respectivamente. Tales valores límites de $e$ e $i$ son seleccionados al azar. Nuestros resultados muestran que fragmentos de Plutinos con diámetros $D>1 \mathrm{~km}$ requieren escalas de tiempo del orden de $10^{8}$ años para alcanzar la configuración orbital actual (Figura 7.8), mientras que fragmentos más pequeños 

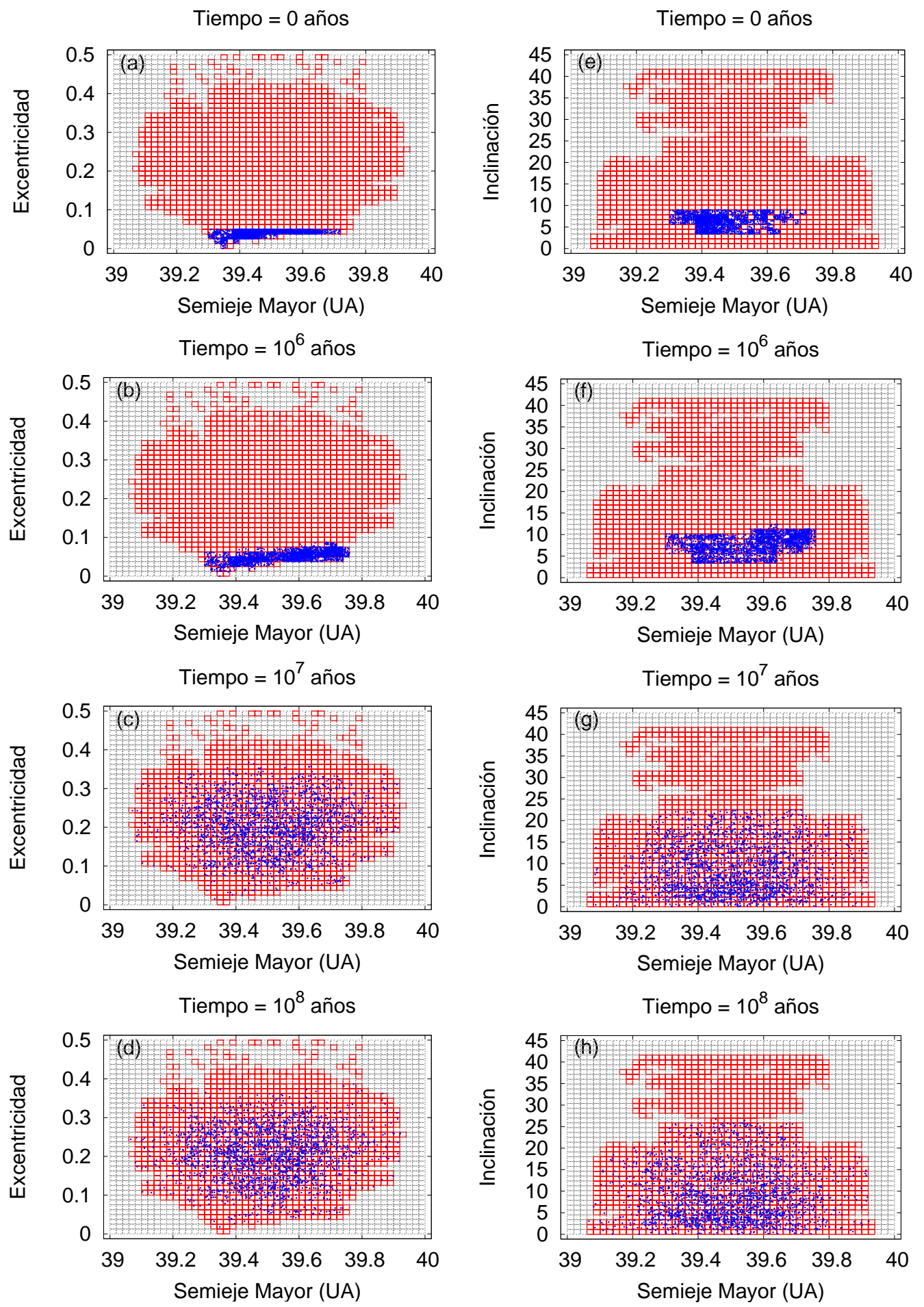

Figura 7.8. Distribución de fragmentos de Plutinos con diámetros $D>1 \mathrm{~km}$ con respecto al semieje mayor $a$, excentricidad $e$ e inclinación $i$, como función del tiempo. 
ocupan los nichos de estabilidad muy rápidamente, en sólo algunos miles de años. Además, encontramos que los resultados concernientes a la distribución de tamaños de los Plutinos, sus tiempos de vida colisionales medios, la tasa de eyección de fragmentos desde la resonancia 3:2 con Neptuno y la formación de familias no muestran una fuerte dependencia sobre la distribución inicial de elementos orbitales. A partir de este análisis, inferimos que la distribución orbital actual de los Plutinos no ofrece una fuerte restricción sobre el origen dinámico de esta población. 



\section{Capítulo 8}

\section{Conclusiones}

En este trabajo, hemos analizado la evolución colisional y dinámica de las principales poblaciones de pequeños planetas del Sistema Solar. Nuestro estudio ha incluido a los asteroides del Cinturón Principal, los asteroides cercanos a la Tierra (NEAs), los Troyanos jovianos asociados al punto $L_{4}$ y los Plutinos.

Para realizar esta tarea, hemos desarrollado un algoritmo numérico estadístico que incluye procesos colisionales y los principales mecanismos dinámicos asociados a las poblaciones bajo estudio.

Los resultados más importantes derivados en esta Tesis se resumen a continuación:

\section{Asteroides del Cinturón Principal y NEAs}

- La distribución de tamaños del Cinturón Principal de asteroides está determinada fundamentalmente por procesos colisionales, aunque la acción del efecto Yarkovsky y las resonancias orbitales también juegan un rol relevante.

- La población del Cinturón Principal de asteroides es preferentemente un "fósil", adquiriendo una suave estructura de onda similar a la actual durante los primeros $5 \times$ $10^{6}$ años de evolución. Este resultado es consistente con lo sugerido por Bottke et al. (2005a).

- La distribución de tamaños de los NEAs está determinada fundamentalmente por la remoción dinámica de asteroides desde el Cinturón Principal, debida a la acción del efecto Yarkovsky y las resonancias orbitales. Por otra parte, los procesos colisionales no juegan un rol relevante en la determinación cuantitativa de la población de NEAs.

- El efecto Yarkovsky es el proceso más importante que remueve material desde el Cinturón Principal hacia la zona de los NEAs, a través de las resonancias orbitales. Este resultado es consistente con aquello sugerido por Morbidelli et al. (2002) y Morbidelli \& Vokrouhlický (2003). Por otra parte, la inyección colisional de fragmentos asteroidales dentro de las resonancias poderosas del Cinturón Principal resulta tener un efecto menor. 
- Un objeto del tamaño de Vesta, perteneciente al anillo Interior del Cinturón Principal de asteroides, tiene un $\sim 75 \%$ de probabilidad de sobrevivir sin recibir un impacto catastrófico sobre la edad del Sistema Solar. Este resultado es consistente con la preservación de la corteza basáltica de dicho asteroide.

- 8 grandes familias de asteroides son formadas a partir de la fractura de objetos con diámetros mayores a los $200 \mathrm{~km}$. Este resultado está en absoluto acuerdo con aquello sugerido por Davis et al. (1985).

- Los tiempos de vida colisionales medios de objetos de 1 metro de diámetro pertenecientes a los anillos Interior, Medio y Exterior del Cinturón Principal son de 3.2, 4.3 y 6.8 $\times 10^{6}$ años, respectivamente. Estas estimaciones son consistentes con los tiempos de exposición a los rayos cósmicos para los diferentes tipos de condritas ordinarias.

- La distribución y mezcla de clases taxonómicas observadas en el Cinturón Principal de asteroides no pueden ser explicadas por el intercambio colisional de masa, de modo que tal distribución de taxonomías debería ser una característica primordial de esta población, asociada a su proceso de formación.

- Aproximadamente el $94 \%$ de la población de NEAs proviene de los anillos Interior y Medio del Cinturón Principal ( $a \lesssim 2.8$ UA), mientras que sólo el $6 \%$ restante tiene su origen en el anillo Exterior $(a \gtrsim 2.8 \mathrm{UA})$. Este resultado es consistente con aquello discutido por Bottke et al. (2002).

\section{Troyanos de Júpiter}

- Los resultados de nuestras simulaciones muestran ondas en las distribuciones de tamaños evolucionadas finales de los Troyanos jovianos de $L_{4}$, las cuales se propagan desde diámetros $D$ de $\sim 0.1 \mathrm{a} \sim 60 \mathrm{~km}$. En términos generales, los más grandes gaps entre la ley $Q_{\mathrm{S}}$ y la ley $Q_{\mathrm{D}}$ de Benz \& Asphaug (1999) para cuerpos de hielo a $3 \mathrm{~km} \mathrm{~s}{ }^{-1}$, llevan a las más grandes amplitudes de onda. Por otra parte, nuestros resultados indican que cuanto menor es la masa de la población inicial, mejor se reproduce la distribución de tamaños observada de los Troyanos jovianos de $L_{4}$.

- Los tiempos de vida colisionales medios de la población de $L_{4}$ indican que los asteroides Troyanos jovianos de mayor tamaño probablemente han sobrevivido inalterados por eventos catastróficos sobre la edad del Sistema Solar. Este resultado es consistente con aquello discutido por Davis \& Weidenschilling (1981) y Marzari et al. (1997).

- Las simulaciones con los más grandes gaps entre las leyes $Q_{\mathrm{S}}$ y $Q_{\mathrm{D}}$ no forman ninguna familia a partir de la fractura de cuerpos con diámetros mayores a los 50 y $100 \mathrm{~km}$ que dispersen fragmentos del orden de los $40 \mathrm{~km}$, sobre la edad del Sistema Solar. Por otra parte, usando una ley $Q_{\mathrm{S}}$ particular con un pequeño gap respecto de $Q_{\mathrm{D}}$, es posible derivar resultados consistentes con el número de familias de Troyanos de $L_{4}$ encontradas por Beaugé \& Roig (2001), Dotto et al. (2006) y Fornasier et al. (2007), sugiriendo además que nuevas familias deberían ser identificadas en el futuro.

- La tasa máxima de eyección de fragmentos desde el enjambre joviano $L_{4}$, correspondiente a los más pequeños gaps entre las leyes $Q_{\mathrm{S}}$ y $Q_{\mathrm{D}}$, es de $\sim 50$ objetos con diámetros 
mayores a $1 \mathrm{~km}$ por millón de años. Nuestra estimación resulta ser significativamente menor que aquella obtenida por Marzari et al. (1997), quienes derivaron una tasa de eyección colisional de $\sim 3600$ objetos con diámetros entre 1 y $40 \mathrm{~km}$ por millón de años desde el mismo enjambre. A partir de nuestros estudio, un máximo de 1 Centauro o Cometa de la Familia de Júpiter sería esperado cada 20000 años desde la población de Troyanos jovianos de $L_{4}$. Concluimos que la contribución de los asteroides Troyanos jovianos a las poblaciones actuales de Centauros y Cometas de la Familia de Júpiter resulta ser despreciable, en comparación con aquella proporcionada por el Disco Dispersado de la Región Transneptuniana (Di Sisto \& Brunini 2007).

- La luminosidad del polvo en el enjambre joviano $L_{4}$ resulta ser comparable a la luminosidad del polvo en el Sistema Solar interior y, al menos, un orden de magnitud más baja que la luminosidad del polvo asociado al Sistema Solar exterior.

- La distribución orbital actual de los asteroides Troyanos jovianos de $L_{4}$ no ofrece una fuerte restricción sobre el origen dinámico de esta población.

\section{Plutinos}

- Una población inicial modelada a través de una ley de potencias con una única pendiente no permite reproducir el quiebre sugerido por Bernstein et al. (2004) y Kenyon et al. (2008) a un diámetro cercano a 40-80 km en la distribución de tamaños acumulada de los Plutinos. Remarcamos que los resultados de Petit et al. (2006) sugieren que la existencia de un quiebre tal en la distribución de objetos transneptunianos es aun una materia de debate.

- La población de Plutinos con diámetros mayores a unos pocos kilómetros no es significativamente alterada por impactos catastróficos sobre la edad del Sistema Solar. De este modo, la posible existencia de un quiebre a un diámetro cercano a 40-80 km en la distribución de tamaños acumulada de los Plutinos debería ser un rasgo primordial de la población y no el resultado de la evolución colisional. Esto concuerda con el análisis de Charnoz \& Morbidelli (2007), quienes argumentaron que el quiebre en la distribución de los objetos del Cinturón de Kuiper alrededor de los 50 km debería ser primordial.

- 7 grandes familias son formadas en la resonancia de movimientos medios 3:2 con Neptuno sobre la edad del Sistema Solar, a partir de la fractura de un cuerpo con un diámetro mayor a $100 \mathrm{~km}$, dispersando fragmentos del orden de los 30-40 km.

- Un objeto con una diámetro mayor a $1 \mathrm{~km}$ es eyectado desde la resonancia 3:2 con Neptuno cada 400-1 700 años. Tales objetos eyectados resultan ser fragmentos con diámetros entre 1 y $5 \mathrm{~km}$, resultantes de impactos entre cuerpos precursores con diámetros entre 10 y $200 \mathrm{~km}$.

- Cuanto más grande es el número de objetos del tamaño de Plutón presentes en esta población, mayor es la tasa de eyección de fragmentos desde aquella región resonante y el número de familias formadas sobre $4.5 \times 10^{9}$ años. En efecto, si asumimos que un máximo de 5 objetos del tamaño de Plutón están presentes en la resonancia 3:2 (Sección 7.2), un cuerpo con un diámetro mayor a $1 \mathrm{~km}$ es eyectado cada algunas decenas de años mientras que $\sim 170$ grandes familias son formadas sobre la historia del Sistema Solar. 
De acuerdo a los resultados de Di Sisto \& Brunini (2007), concluimos que es necesario especificar el número de objetos del tamaño de Plutón presentes en la resonancia 3:2 con Neptuno, para determinar si esta región resonante puede ser considerada como una fuente importante de Cometas Eclípticos.

- La distribución orbital actual de los Plutinos no ofrece una fuerte restricción sobre el origen dinámico de esta población. 


\section{Bibliografía}

Aitken, R. G. 1926, Publications of the Astronomical Society of the Pacific, 38, 376

Alvarez, L. W., Alvarez, W., Asaro, F., \& Michel, H. V. 1980, Science, 208, 1095

Backman D. E., \& Paresce F. 1993, Protostars and Planets III, (Levy, E. H., \& Lunine, J. I., Eds.), University of Arizona Press, Tucson, AZ, 1253

Barucci, M. A., \& Peixinho, N. 2006, Asteroids, Comets, Meteors, Proceedings of the 229th Symposium of the International Astronomical Union, (Lazzaro, D., Ferraz-Melo, S., \& Fernández, J. A., Eds.), Cambridge University Press, Cambridge, 171

Beaugé, C. 1994, Celestial Mechanics and Dynamical Astronomy, 60, 225

Beaugé, C., \& Roig, F. 2001, Icarus, 153, 391

Bell, J. F. 1986, Lunar and Planetary Science XVII, 985

Bell, J. F., Davis, D. R., Hartmann, W. K., \& Gaffey, M. J. 1989, Asteroids II, (Binzel, R. P., Gehrels, T., \& Matthews, M. S., Eds.), University of Arizona Press, Tucson, AZ, 921

Benz, W., \& Asphaug, E. 1999, Icarus, 142, 5

Bernstein, G. M., Trilling, D. E., Allen, R. L., et al. 2004, AJ, 128, 1364

Bien, R., \& Schubart, J. 1987, A\&A, 175, 292

Binzel, R. P., \& Sauter, L. M. 1992, Icarus, 95, 222

Bottke, W. F., Morbidelli, A., Jedicke, R., et al. 2002, Icarus, 156, 399

Bottke, W. F., Nolan, M. C., Greenberg, R., \& Kolvoord, R. A. 1994, Icarus, 107, 255

Bottke, W. F., Durda, D. D., Nesvorný, D., Jedicke, R., Morbidelli, A., et al. 2005a, Icarus, 175,111

Bottke, W. F., Durda, D. D., Nesvorný, D., Jedicke, R., Morbidelli, A., et al. 2005b, Icarus, 179,63

Bowell, E., Hapke, B., Domingue, D., et al. 1989, Asteroids II, (Binzel, R. P., Gehrels, T., \& Matthews, M. S., Eds.), University of Arizona Press, Tucson, AZ, 524

Brown, M. E. 2001, AJ, 121, 2804 
Brown, M. E., Bouchez, A. H., Rabinowitz, D. L., Sari, R., Trujillo, C. A., et al. 2005a, ApJ, 632, L45

Brown, M. E., Trujillo, C. A., \& Rabinowitz, D. L. 2005b, ApJ, 635, L97

Brown, M. E., Schaller, E. L., Roe, H. G., Rabinowitz, D. L., \& Trujillo, C. A. 2006, ApJ, 643, L61

Brown, M. E. 2008, The Solar System Beyond Neptune, (Barucci, M. A., Boehnhardt, H., Cruikshank, D. P., \& Morbidelli, A., Eds.), University of Arizona Press, Tucson, AZ, 335

Brož, M., Vokrouhlický, D., Roig, F., Nesvorný, D., Bottke, W. F., et al. 2005, MNRAS, 359, 1437

Brunini, A., \& Fernández, J. A. 1999, Planetary and Space Science, 47, 591

Brunini, A., Di Sisto, R. P., \& Orellana, R. B. 2003, Icarus, 165, 371

Buie, M. W., Grundy, W. M., Young, E. F., Young, L. A., \& Stern, S. A. 2006 AJ, 132, 290

Burns, J. A., Lamy, P. L., \& Soter, S. 1979, Icarus, 40, 1

Caffee, M. W., Reedy, R. C., Goswami, J. N., Hohenberg, C. M., \& Marti, K. 1988, Meteorites and the Early Solar System, (Kerridge, J. F., \& Matthews, M. S., Eds.), University of Arizona Press, Tucson, AZ, 205

Campbell, W. W. 1916, Publications of the Astronomical Society of the Pacific, 28, 222

Campo Bagatin, A. 1998, Ph. D. Thesis, University of Valencia

Campo Bagatin, A., Cellino, A., Davis, D. R., Farinella, P., \& Paolicchi, P. 1994a, Planetary and Space Science, 42, 1079

Campo Bagatin, A., Farinella, P., \& Petit, J. 1994b, Planetary and Space Science, 42, 1099

Charnoz, S., \& Morbidelli, A. 2007, Icarus, 188, 468

Chyba, C. F., Thomas, P. J., \& Zahnle, K. J. 1993, Nature, 361, 40

Dahlgren, M. 1998, A\&A, 336, 1056

Davies, J. K., McFarland, J., Bailey, M. E., Marsden, B. G., \& Ip, W. 2008, The Solar System Beyond Neptune, (Barucci, M. A., Boehnhardt, H., Cruikshank, D. P., \& Morbidelli, A., Eds.), University of Arizona Press, Tucson, AZ, 11

Davis, D. R., Chapman, C. R., Greenberg, R., Weidenschilling, S. J., \& Harris, A. W. 1979, Asteroids, (Gehrels, T., Ed.), University of Arizona Press, Tucson, AZ, 528

Davis, D. R., \& Weidenschilling, S. J. 1981, Lunar and Planetary Science XII, 199

Davis, D. R., Chapman, C. R., Weidenschilling, S. J., \& Greenberg, R. 1985, Icarus, 62, 30

Davis, D. R., Weidenschilling, S. J., Farinella, P., Paolicchi, P., \& Binzel, R. P. 1989, Asteroids II, (Binzel, R. P., Gehrels, T., \& Matthews, M. S., Eds.), University of Arizona Press, Tucson, AZ, 805 
Davis, D. R., Ryan, E. V., \& Farinella, P. 1994, Planetary and Space Science, 42, 599

Davis, D. R., Ryan, E. V., \& Farinella, P. 1995, Lunar and Planetary Science Conference, 26,319

de Elía, G. C., \& Brunini, A. 2007a, A\&A, 466, 1159

de Elía, G. C., \& Brunini, A. 2007b, A\&A, 475, 375

de Elía, G. C., Brunini, A., \& Di Sisto R. P. 2008, A\&A, 490, 835

Dell'Oro, A., \& Paolicchi, P. 1998, Icarus, 136, 328

Dell'Oro, A., Paolicchi, P., Marzari, F., Dotto, E., \& Vanzani, V. 1998, A\&A, 339, 272

Dell'Oro, A., Marzari, F., Paolicchi, P, \& Vanzani, V. 2001, A\&A, 366, 1053

Dermott S. F., Jayaraman S., Xu Y. L., Gustafson B. A. S., \& Liou, J. C. 1994, Nature, 369,719

Dermott S. F., Kehoe T. J. J., Durda D. D., Grogan K., \& Nesvorný, D. 2002, Proceedings of Asteroids, Comets, Meteors - ACM 2002, (B. Warmbein, Ed.), ESA SP-500. Noordwijk, Netherlands: ESA Publications Division, 319

Di Sisto, R. P., \& Brunini, A. 2007, Icarus, 190, 224

Dobrovolskis, A. R., \& Burns, J. A. 1984, Icarus, 57, 464

Dohnanyi, J. W. 1969, Journal of Geophysical Research, 74, 2531

Doressoundiram, A., Peixinho, N., de Bergh, C, Fornasier, S., Thébault, P., Barucci, M. A., \& Veillet, C. 2002, AJ, 124, 2279

Doressoundiram, A., Peixinho, N., Doucet, C, Mousis, O., Barucci, M. A., Petit, J. M., \& Veillet, C. 2005, Icarus, 174, 90

Dotto, E., Fornasier, S., Barucci, M. A., Licandro, J., Boehnhardt, H., et al. 2006, Icarus, 183,420

Duncan, M. J., Quinn, T., \& Tremaine, S. 1988, ApJ. Lett., 328, L69

Duncan, M. J., Levison, H. F., \& Budd, S. M. 1995, AJ, 110, 3073

Duncan, M. J., \& Levison, H. F. 1997, Science, 276, 1670

Durda, D. D. 1993, Ph.D. Thesis, University of Florida

Durda, D. D., \& Dermott, S. F. 1997, Icarus, 130, 140

Durda, D. D., Greenberg, R., \& Jedicke, R. 1998, Icarus, 135, 431

Edgeworth, K. E. 1938, Trustees of the National Library of Ireland, Dublin. Manuscript Nos. $16869 / 47$ y /48, 226

Edgeworth, K. E. 1943, Journal of the British Astronomical Association, 53, 181 
Edgeworth, K. E. 1949, MNRAS, 109, 600

Elliot, J. L., Kern, S. D., Clancy, K. B., et al. 2005, AJ, 129, 1117

Érdi, B. 1978, Celestial Mechanics, 18, 141

Everhart, E. 1972, Astrophysical Letters, 10, 131

Everhart, E. 1977, Comets, asteroids, meteorites: Interrelations, evolution and origins; Proceedings of the Thirty-ninth International Colloquium, Lyons, France, 99

Farinella, P., \& Davis, D. R. 1992, Icarus, 97, 111

Farinella, P., Paolicchi, P., \& Zappalà, V. 1982, Icarus, 52, 409

Farinella, P., Vokrouhlický, D., \& Hartmann, W. K. 1998, Icarus, 132, 378

Fernández, J. A. 1980, MNRAS, 192, 481

Fernández, J. A., Tancredi, G., Rickman, H., \& Licandro, J. 1999, A\&A, 352, 327

Fernández, J. A., Gallardo, T., \& Brunini, A. 2002, Icarus, 159, 358

Fernández, Y. R., Sheppard, S. S., \& Jewitt, D. J. 2003, AJ, 126, 1563

Fitzsimmons, A., Dahlgren, M., Lagerkvist, C. I., Magnusson, P., \& Williams, I. P. 1994, A\&A, 282, 634

Foderà Serio, G., Manara, A., \& Sicoli, P. 2002, Asteroids III, (Bottke, W. F., Cellino, A., Paolicchi, P., \& Binzel, R. P., Eds.), University of Arizona Press, Tucson, AZ, 17

Fornasier, S., Dotto, E., Marzari, F., Barucci, M. A., Boehnhardt, H., Hainaut, O., \& de Bergh, C. 2004, Icarus, 172, 221

Fornasier, S., Dotto, E., Hainaut, O., Marzari, F., Boehnhardt, H., de Luise, F., \& Barucci, M. A. 2007, Icarus, 190, 622

Fujiwara, A., \& Tsukamoto, A. 1980, Icarus, 44, 142

Fujiwara, A., Kamimoto, G., \& Tsukamoto, A. 1977, Icarus, 31, 277

Gault, D. E., Shoemaker, E. M., \& Moore, H. J. 1963, NASA Tech. Note D-1767

Giblin, I., Martelli, G., Smith, P. N., et al. 1994, Icarus, 110, 203

Giblin, I. 1998, Planetary and Space Science, 46, 921

Gil-Hutton, R., \& Brunini, A. 2000, Icarus, 145, 382

Gladman, B. J., Migliorini, F., Morbidelli, A., Zappalà, V., Michel, P., Cellino, A., Froeschle, C., Levison, H. F., Bailey, M., \& Duncan, M. 1997, Science, 277, 197

Gladman, B. J., Holman, M., Grav, T., Kavelaars, J., Nicholson, P., Aksnes, K., \& Petit, J. M. 2002, Icarus, 157, 269 
Gradie, J., \& Tedesco, E. 1982, Science, 216, 1405

Greenberg, R., Hartmann, W. K., Chapman, C. R., \& Wacker, J. F. 1978, Icarus, 35, 1

Greenberg, R., \& Nolan, M. C. 1989, Asteroids II, (Binzel, R. P., Gehrels, T., \& Matthews, M. S., Eds.), University of Arizona Press, Tucson, AZ, 778

Grundy, W. M., Noll, K. S., \& Stephens, D. C. 2005, Icarus, 176, 184

Grundy, W. M., Stansberry, J. A., Noll, K. S., Stephens, D. C., Trilling, D. E., Kern, S. D., Spencer, J. R., Cruikshank, D. P., \& Levison, H. F. 2007, Icarus, 191, 286

Grundy, W. M., Noll, K. S., Virtanen, J., Muinonen, K., Kern, S. D., Stephens, D. C., Stansberry, J. A., Levison, H. F., \& Spencer, J. R. 2008, Icarus, 197, 260

Guillens, S. A., Vieira Martins, R., \& Gomes, R. S. 2002, AJ, 124, 2322

Harris, A. W. 2002, Bulletin of the American Astronomical Society, 34, 835

Hartmann, W. K., Tholen, D. J., \& Cruikshank, D. P. 1987, Icarus, 69, 33

Hartmann, W. K. 1988, Lunar and Planetary Science XIX, 451

Hartmann, W. K., Ryder, G., Dones, L., \& Grinspoon, D. 2000, Origin of the earth and moon, (Canup, R. M., \& Righter, K., Eds.), University of Arizona Press, Tucson, AZ, 493

Hirayama, K. 1918, AJ, 31, 185

Holsapple, K. A. 1993, Annual Review of Earth and Planetary Sciences, 21, 333

Holsapple, K. A. 1994, Planetary and Space Science, 42, 1067

Housen, K. R. 1991, Icarus, 94, 180

Housen, K. R., \& Holsapple, K. A. 1990, Icarus, 84, 226

Housen, K. R., \& Holsapple, K. A. 1999, Icarus, 142, 21

Ivezić, Ž., Tabachnik, S., Rafikov, R., et al., and the SDSS Collaboration 2001, AJ, 122, 2749

Jedicke, R., \& Metcalfe, T. S. 1998, Icarus, 131, 245

Jewitt, D. C., \& Luu, J. X. 1990, AJ, 100, 933

Jewitt, D. C., \& Luu, J. X. 1992, IAU Circular 5611, 1

Jewitt, D., \& Luu, J. 1993, Nature, 362, 730

Jewitt, D. C., Trujillo, C. A., \& Luu, J. X. 2000, AJ, 120, 1140

Jewitt, D. C., \& Sheppard, S. S. 2002, AJ, 123, 2110

Joss, P. C. 1973, A\&A, 25, 271

Keil, K. 2002, Asteroids III, (Bottke, W. F., Cellino, A., Paolicchi, P., \& Binzel, R. P., Eds.), University of Arizona Press, Tucson, AZ, 573 
Kepler, J. 1596, Prodromus dissertationum cosmographicarum continents mysterium cosmographicum de admirabili proportione orbium celestium de que causis coelorum numeri, magnitudinis motuumque periodicorum genuinis et propiis, demonstratum per quinqueregularia corpora geometrica. Excudebat Georgius Gruppenbachius, Tubingae. Traducido por Duncan, A. M. 1981, The Secret of the Universe, Abaris, Nueva York, Estados Unidos, 267

Kenyon, S. J., Bromley, B. C., O’Brien, D. P., \& Davis, D. R. 2008, The Solar System Beyond Neptune, (Barucci, M. A., Boehnhardt, H., Cruikshank, D. P., \& Morbidelli, A., Eds.), University of Arizona Press, Tucson, AZ, 293

Kirkwood, D. 1867, Meteoric astronomy: a treatise on shooting-stars, fire-balls, and aerolites. Philadelphia, Lippincott \& Co., 129

Klačka, J. 1992, Earth, Moon, and Planets, 59, 41

Knežević, Z., Milani, A., Farinella, P., Froeschlé, Ch, \& Froeschlé, C. 1991, Icarus, 93, 316

Knežević, Z., \& Milani, A. 2000, Celestial Mechanics and Dynamical Astronomy, 78, 17

Knuth, D. E. 1981, The Art of Computer Programming. Volume 2: Seminumerical Algorithms, Reading, Mass.: Addison-Wesley, 2nd ed., 688

Kozai, Y. 1962, AJ, 67, 591

Kring, D. A. 2007, Guidebook to the Geology of Barringer Meteorite Crater, Arizona, LPI Contribution $\mathrm{N}^{\circ} 1355$

Kuiper, G. P. 1951a, Proceedings of a topical symposium, commemorating the 50th anniversary of the Yerkes Observatory and half a century of progress in astrophysics, New York: McGraw-Hill, (Hynek, J. A., Ed.), 357

Kuiper, G. P. 1951b, Proceedings of the National Academy of Sciences of the United States of America, 37, 1

Kuiper, G. P. 1956, Journal of the Royal Astronomical Society of Canada, 50, 158

Lacerda, P., \& Luu, L. 2006, AJ, 131, 2314

Lagrange, J.-L. 1772, Prix de l'Académie Royale des Sciences de Paris, Tome IX; reprinted in 1873, Oeuvres de Lagrange, Tome sixième (J.-A. Serret, Ed.), Gauthiers-Villars, Paris, 229

Landgraf M., Liou J. C., Zook H. A., \& Grün, E. 2002, AJ, 123, 2857

Leuschner, A. O. 1927, Publications of the Astronomical Society of the Pacific, 39, 275

Levison, H., \& Stern, H. 1995, Nature, 385, 42

Levison, H., \& Stern, H. 2001, AJ, 121, 1730

Levison, H., Shoemaker, E. M., \& Shoemaker, C. S. 1997, Nature, 385, 42

Levison, H., \& Morbidelli, A. 2007, Icarus, 189, 196 
Liou J. C., Dermott S. F., \& Xu, Y. L. 1995, Planetary and Space Science, 43, 717

Love, S. G., \& Ahrens, T. J. 1996, Icarus, 124, 141

Lowry, S., Fitzsimmons, A., Lamy, P., \& Weissman, P. 2008 The Solar System Beyond Neptune, (Barucci, M. A., Boehnhardt, H., Cruikshank, D. P., \& Morbidelli, A., Eds.), University of Arizona Press, Tucson, AZ, 397

Marchis, F. and 17 colleagues 2006a, Nature, 439, 565

Marchis, F., Wong, M. H., Berthier, J., Descamps, P., Hestroffer, D., Vachier, F., Le Mignant, D., \& De Pater, I. 2006b, IAU Circular 8732, 1

Marti, K., \& Graf, T. 1992, Annual Review of Earth and Planetary Sciences, 20, 221

Marzari, F., Farinella, P., \& Vanzani, V. 1995, A\&A, 299, 267

Marzari, F., Scholl, H., \& Farinella, P. 1996, Icarus, 119, 192

Marzari, F., Farinella, P., Davis, D. R., Scholl, H., \& Campo Bagatin, A. 1997, Icarus, 125, 39

Marzari, F., Scholl, H., Murray, C., \& Lagerkvist, C. 2002, Asteroids III, (Bottke, W. F., Cellino, A., Paolicchi, P., \& Binzel, R. P., Eds.), University of Arizona Press, Tucson, AZ, 725

Marzari, F., Tricarico, P., \& Scholl, H. 2003, Icarus, 162, 453

McCord, T. B., Adams, J. B., \& Johnson, T. V. 1970, Science, 178, 745

McKinnon, W. B., Prialnik, D., Stern, S. A., \& Coradini, A. 2008, The Solar System Beyond Neptune, (Barucci, M. A., Boehnhardt, H., Cruikshank, D. P., \& Morbidelli, A., Eds.), University of Arizona Press, Tucson, AZ, 213

Melita, M. D., \& Brunini, A. 2000, Icarus, 147, 205

Melosh, H. J. 1989, Impact cratering: A geologic process, Oxford University Press, New York

Melosh, H. J., \& Ryan, E. V. 1997, Icarus, 129, 562

Merline, W. J., Close, L. M., Menard, F., Dumas, C., Chapman, C. R., \& Slater, D. C. 2001, Bulletin of the American Astronomical Society, 33, 1133

Merline, W. J., Weidenschilling, S. J., Durda, D. D., Margot, J. L., Pravec, P., \& Storrs, A. D. 2002, Asteroids III, (Bottke, W. F., Cellino, A., Paolicchi, P., \& Binzel, R. P., Eds.), University of Arizona Press, Tucson, AZ, 289

Michel, P., \& Thomas, F. 1995, A\&A, 307, 310

Michel, P., Migliorini, F., Morbidelli, A., \& Zappalà, V. 2000, Icarus, 145, 332

Migliorini, F., Michel, P., Morbidelli, A., Nesvorný, D., \& Zappalà, V. 1998, Science, 281, 2022 
Milani, A. 1993, Celestial Mechanics and Dynamical Astronomy, 57, 59

Milani, A. 1994, Asteroids, Comets, Meteors 1993: Proceedings of the 160th International Astronomical Union, Belgirate, Italy, (Milani, A., Di Martino, M., \& Cellino, A., Eds.), IAU Symposium N 160, Kluwer Academic Publishers, Dordrecht, 159

Moons, M., \& Morbidelli, A. 1995, Icarus, 114, 33

Moons, M., Morbidelli, A., \& Migliorini, F. 1998, Icarus, 135, 458

Morbidelli, A. 1997, Icarus, 127, 1

Morbidelli, A., \& Gladman, B. 1998, Meteoritics and Planetary Science, 33, 999

Morbidelli, A., \& Henrard, J. 1991, Celestial Mechanics and Dynamical Astronomy, 51, 169

Morbidelli, A., \& Nesvorný, D. 1999, Icarus, 139, 295

Morbidelli, A., \& Vokrouhlický, D. 2003, Icarus, 163, 120

Morbidelli, A., Bottke, W. F., Froeschlé, Ch., \& Michel, P. 2002, Asteroids III, (Bottke, W. F., Cellino, A., Paolicchi, P., \& Binzel, R. P., Eds.), University of Arizona Press, Tucson, AZ, 409

Morbidelli, A., Emel'yanenko, V. V., \& Levison, H. F. 2004, MNRAS, 355, 935

Morbidelli, A., Levison, H. F., Tsiganis, K., \& Gomes, R. 2005, Nature, 435, 462

Morbidelli, A., Levison, H. F., \& Gomes, R. 2008, The Solar System Beyond Neptune, (Barucci, M. A., Boehnhardt, H., Cruikshank, D. P., \& Morbidelli, A., Eds.), University of Arizona Press, Tucson, AZ, 275

Mothé-Diniz, T., Carvano, J. M., \& Lazzaro, D. 2003, Icarus, 162, 10

Murray, N., Holman, M., \& Potter, M. 1998, AJ, 116, 2583

Murray, C. D., \& Dermott, S. F. 1999, Solar System Dynamics, Cambridge University Press.

Nakamura, A., \& Fujiwara, A. 1991, Icarus, 92, 132

Nakamura, A., Suguiyama, K., \& Fujiwara, A. 1992 Icarus, 100, 127

Nesvorný, D., \& Ferraz-Melo, S. 1997, Icarus, 130, 247

Nesvorný, D., \& Morbidelli, A. 1998, AJ, 116, 3029

Nesvorný, D., \& Morbidelli, A. 1999, Celestial Mechanics and Dynamical Astronomy, 71, 243

Nesvorný, D., \& Roig, F. 2000, Icarus, 148, 282

Nesvorný, D., Roig, F., \& Ferraz-Melo, S. 2000, AJ, 119, 953

Nesvorný, D., \& Roig, F. 2001, Icarus, 150, 104 
Nesvorný, D., Ferraz-Mello, S., Holman, M., \& Morbidelli, A. 2002, Asteroids III, (Bottke, W. F., Cellino, A., Paolicchi, P., \& Binzel, R. P., Eds.), University of Arizona Press, Tucson, AZ, 379

Nesvorný, D., Morbidelli, A., Vokrouhlický, D., Bottke, W. F., \& Brož, M. 2002, Icarus, 157, 155

Nolan, M. C., Asphaug, E., Melosh, H. J., \& Greenberg, R. 1996, Icarus, 124, 359

Noll, K. S. 2006, Asteroids, Comets, Meteors, Proceedings of the 229th Symposium of the International Astronomical Union, (Lazzaro, D., Ferraz-Melo, S., \& Fernández, J. A., Eds.), Cambridge University Press, Cambridge, 301

Noll, K. S., Grundy, W. M., Chiang, E. I., Margot, J. L., \& Kern, S. D. 2008, The Solar System Beyond Neptune, (Barucci, M. A., Boehnhardt, H., Cruikshank, D. P., \& Morbidelli, A., Eds.), University of Arizona Press, Tucson, AZ, 345

O'Brien, D. P., \& Greenberg, R. 2003, Icarus, 164, 334

O’Brien, D. P., \& Greenberg, R. 2005, Icarus, 178, 179

Oort, J. H. 1950, Bulletin of the Astronomical Institutes of the Netherlands, 11, 91

Öpik, E. J. 1951, Proceedings of the Royal Irish Academy, Sect. A, 54, 165

Peixinho, N., Boehnhardt, H., Belskaya, I., Doressoundiram, A., Barucci, M. A., \& Delsanti, A. 2004, Icarus, 170, 153

Penco, U., Dell'Oro, A., La Spina, A., Paolicchi, P., Cellino, A., \& Campo Bagatin, A. 2002, Proceedings of Asteroids, Comets, Meteors - ACM 2002 (Barbara Warmbein, Ed.). International Conference, Berlin, Germany. ESA SP-500. Noordwijk, Netherlands: ESA Publications Division, 363

Peterson, C. 1976, Icarus, 29, 91

Petit, J., \& Farinella, P. 1993, Celestial Mechanics and Dynamical Astronomy, 57, 1

Petit, J., Morbidelli, A., \& Valsecchi, G. B. 1999, Icarus, 141, 367

Petit, J., Morbidelli, A., \& Chambers, J. 2001, Icarus, 153, 338

Petit, J., Holman, M. J., Gladman, B. J., et al. 2006, MNRAS, 365, 429

Piazzi, G. 1801, Risultati delle Osservazioni della Nuova Stella scoperta il dì 1. Gennajo all'Osservatorio reale di Palermo. Nella Reale Stamperia, Palermo, 25

Piotrowski, S. 1953, Acta Astron. Ser. A, 6, 115

Pravec, P., Harris, A. W., \& Michalowski, T. 2002, Asteroids III, (Bottke, W. F., Cellino, A., Paolicchi, P., \& Binzel, R. P., Eds.), University of Arizona Press, Tucson, AZ, 113

Rabe, E. 1967, AJ, 72, 10 
Rabinowitz, D. L., Bowell, E., Shoemaker, E. M., \& Muinonen, K. 1994, Hazards Due to Comets and Asteroids, (Gehrels, T., Matthews, M. S., \& Schumann, A., Eds.), University of Arizona Press, Tucson, AZ, 285

Rabinowitz, D. L., Helin, E., Lawrence, K., \& Pravdo, S. 2000, Nature, 403, 165

Rabinowitz, D. L., Barkume, K., Brown, M. E., Roe, H., Schwartz, M., Tourtellote, S., \& Trujillo, C. 2006, ApJ, 639, 1238

Rubincam, D. P., Rowlands, D. D., \& Ray, R. D. 2002, Journal of Geophysical Research (Planets) 107, 3

Ryan, E. V. 1992, Ph. D. Thesis, University of Arizona

Ryan, E. V., \& Melosh, H. J. 1998, Icarus, 133, 1

Scholl, H., Marzari, F., \& Tricarico, P. 2005, Icarus, 175, 397

Schubart, J., \& Bien, R. 1987, A\&A, 175, 299

Sekanina, Z. 1983, AJ, 88, 1382

Sheppard, S. S., \& Trujillo, C. 2006, Bulletin of the American Astronomical Society, 38, 564

Shoemaker, E. M., Shoemaker, C., \& Wolfe, R. F. 1989, Asteroids II, (Binzel, R. P., Gehrels, T., \& Matthews, M. S., Eds.), University of Arizona Press, Tucson, AZ, 487

Smith, B. A., Soderblom, L. A., Johnson, T. V., Ingersoll, A. P., Collins, S. A., Shoemaker, E. M., Hunt, G. E., Masursky, H., Carr, M. H., Davies, M. E., Cook, A. F., Boyce, J. M., Owen, T., Danielson, G. E., Sagan, C., Beebe, R. F., Veverka, J., McCauley, J. F., Strom, R. G., Morrison, D., Briggs, G. A., \& Suomi, V. E. 1979a, Science, 204, 951

Smith, B. A., Soderblom, L. A., Beebe, R., Boyce, J., Briggs, G., Carr, M., Collins, S. A., Johnson, T. V., Cook, A. F., Danielson, G. E., \& Morrison, D 1979b, Science, 206, 927

Spencer, J. R., Stansberry, J. A., Grundy, W. M., \& Noll, K. S. 2006, Bulletin of the American Astronomical Society, 38, 564

Stansberry, J., Grundy, W. M., Margot J-L, Cruikshank, D. P., Emery, J. P., Rieke, G. H., \& Trilling, D. E. 2006, ApJ, 643, 556

Stansberry, J., Grundy, W., Brown, M., Cruikshank, D., Spencer, J., Trilling, D., \& Margot J-L 2008, The Solar System Beyond Neptune, (Barucci, M. A., Boehnhardt, H., Cruikshank, D. P., \& Morbidelli, A., Eds.), University of Arizona Press, Tucson, AZ, 161

Stern, S. A. 1996, AJ, 112, 1203

Stern, S. A., \& Colwell, J. E. 1997a, AJ, 114, 841

Stern, S. A., \& Colwell, J. E. 1997b, ApJ, 490, 879

Stoeffler, D., Gault, D. E., Wedekind, J., \& Polkowski, G. 1975, Journal of Geophysical Research, 80, 4062 
Stuart, J. S. 2001, Science, 294, 1691

Szabó, Gy. M., Ivezić, Z., Jurić, M, \& Lupton, R. 2007, MNRAS, 377, 1393

Szebehely, V. 1967, New York: Academic Press

Takahashi, S., \& Ip, W. H. 2004, Publications of the Astronomical Society of Japan, 56, 1099

Tanaka, H., Inaba, S., \& Nakazawa, K. 1996, Icarus, 123, 450

Tedesco, E. F. 1989, Asteroids II, (Binzel, R. P., Gehrels, T., \& Matthews, M. S., Eds.), University of Arizona Press, Tucson, AZ, 1090

Tholen, D. J. 1984, Ph.D. Thesis, University of Arizona

Thomas, F., \& Morbidelli, A. 1996, Celestial Mechanics and Dynamical Astronomy, 64, 209

Thomas, P. C., Binzel, R. P., Gaffey, M. J., Storrs, A. D., Wells, E. N., \& Zellner, B. H. 1997, Science, 277, 1492

Tsiganis, K., Gomes, R., Morbidelli, A., Levison, H. 2005, Nature, 435, 459

Turco, R. P., Toon, O. B., Park, C., Whitten, R. C., Pollack, J. B., \& Noerdlinger, P. 1982, Icarus, 50, 1

Vasilyev, N. V. 1998, Planetary and Space Science, 46, 129

Vokrouhlický, D., \& Čapek, D. 2002, Icarus, 159, 449

Vokrouhlický, D., Brož, M., Bottke, W. F., Nesvorný, D., \& Morbidelli, A. 2006, Icarus, 182, 118

Weidenschilling, S. J. 1977, Ap\&SS, 51, 153

Welten, K. C., Lindner, L., van der Borg, K., Loeken, T, Scherer, P., \& Schultz, L. 1997, Meteoritics and Planetary Science, 32, 891

Wetherill, G. W. 1979, Icarus, 37, 96

Williams, D. R., \& Wetherill, G. W. 1994, Icarus, 107, 117

Williams, J. G. 1969, Ph. D. Thesis, University of California

Williams, J. G., Faulkner, J. 1981, Icarus, 46, 390

Wisdom, J. 1983, Icarus, 56, 51

Wisdom, J. 1985a, Icarus, 63, 272

Wisdom, J. 1985b, Nature, 315, 731

Yamamoto S., \& Mukai, T. 1998, A\&A, 329, 785

Yoshida, F., Nakamura, T., Watanabe, J., Kinoshita, D., Yamamoto, N., \& Fuse, T. 2003, Publications of the Astronomical Society of Japan, 55, 701 
Yoshida, F., \& Nakamura, T. 2005, AJ, 130, 2900

Yu, Q., \& Tremaine, S. 1999, AJ, 118, 1873

Zappalà, V., Bendjoya, P., Cellino, A., Farinella, P., \& Froeschlé, Ch. 1995, Icarus, 116, 291 